Universidade de Brasília

Instituto de Ciências Exatas

Departamento de Matemática

Existência de soluções positivas para equações e sistemas semilineares via fundamentos topológicos e baricentro

por

Elson Leal de Moura 
"O futuro tem muitos nomes: para os fracos, é o inatingivel; para os temerosos, o desconhecido; para os valentes, é a oportunidade." 
Universidade de Brasília

Instituto de Ciências Exatas

Departamento de Matemática

\section{Existência de soluções positivas para equações e sistemas semilineares via fundamentos topológicos e baricentro por}

\section{Elson Leal de Moura}

Tese apresentada ao Corpo Docente do Programa de Pós-Graduação em Matemática-UnB, como requisito parcial para obtenção do grau de

\section{DOUTOR EM MATEMÁTICA}

Brasília, 09 de março de 2017.

Comissão Examinadora:

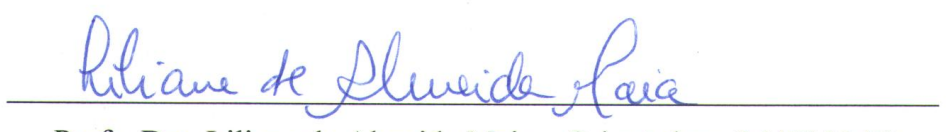

Profa. Dra. Liliane de Almeida Maia - Orientadora (MAT-UnB)

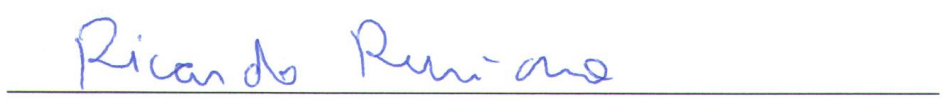

Prof. Dr. Ricardo Ruviaro (MAT-UnB)

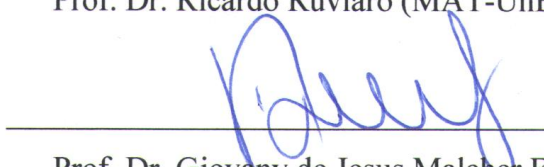

Prof. Dr. Giovany de Jesus Malcher Figueiredo (MAT-UnB)

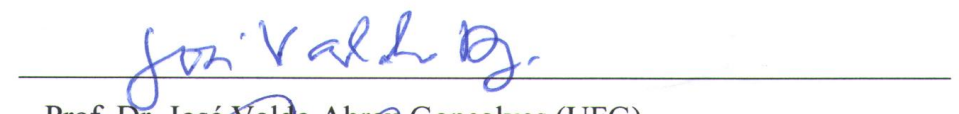

Prof. Dr. José Valdo Abreq Gonçalves (UFG)

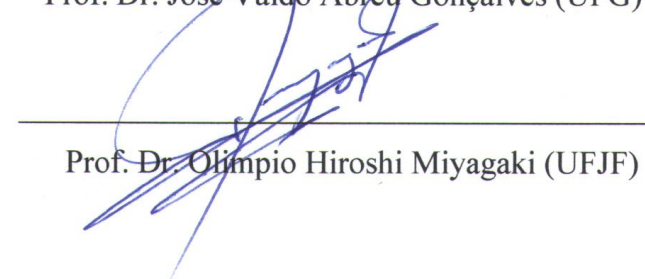

*O autor foi bolsista CAPES durante a elaboração deste trabalho. 
Ficha catalográfica elaborada automaticamente, com os dados fornecidos pelo(a) autor(a)

Leal de Moura, Elson
Existência de soluções positivas para equações e
sistemas semilineares via fundamentos topológicos e
baricentro. / Elson Leal de Moura; orientador
Liliane Almeida Maia. -- Brasilia, 2017.
112 p.
Tese (Doutorado - Doutorado em Matemática) --
Universidade de Brasilia, 2017.
1. Equacões de Schrodinger. 2. Sistema fortemente
acoplado. 3. Assintoticamente linear e superlinear.
4. Decaimento exponencial. 5. Métodos variacionais.
I. Almeida Maia, Liliane, orient. II. Título.




\section{Agradecimentos}

Ao meu Senhor, Criador, Pai, Redentor. A Ele dou graças sempre. Em especial, neste momento, agradeço por esta grandiosa conquista.

A minha mãe Maria, Nossa Senhora Aparecida, eu te escolhi como Padroeira e Defensora dos meus estudos. Iluminou com Tua claridade as obscuridades da minha inteligência, foi e será o meu guia. Não cabe mais palavras Mãe Santíssima, apenas o silêncio de meu coração feliz e agradecido.

Ao meu filho Bernardo que mesmo longe de mim torceu para eu chegar a este dia. A você, meu filho amado, dedico esta tese. A você dedico todos os meus dias de vida.

A minha família: Elza, Pai(falecido em 2016), Elcio, Elisiane, Ednéia, Júnior que estão sempre comigo espiritualmente. Nós conseguimos!

A meus dois grandes amigos que a Vida me permitiu conhecer e aprender com eles : "João Olímpio Cardoso" (falecido em 2016) e "José Geraldo Alves de Amaral". Obrigado por tudo!

A minha avó, tios, tias e primos que rezaram por mim. A graça de Deus esteja convosco.

A minha orientadora, professora Liliane, obrigado pela orientação no Mestrado e Doutorado, por me ensinar a ser melhor do que eu era. Peço desculpas pelos meus erros.

Ao professor Olímpio, por me incentivar a estudar e crescer. Obrigado pela amizade e felicidade de permitir conhecer uma pessoa de tão grande coração. Que Deus lhe dê muitas graças e bençãos.

Ao professor Ricardo, obrigado pela amizade e disponibilidade para colaborar com a minha tese. Obrigado grande amigo!

Aos professores José Valdo e Giovany Figueiredo por participarem da banca, pelas correções e pelas sugestões para a finalização deste trabalho.

Aos amigos Adriano, Camila, Cid e Dióscoros por terem tornado esse período mais agradável.

Agradeço a UFVJM pelo apoio financeiro durante este trabalho. 


\section{Resumo}

O problema semilinear elíptico

$$
\left\{\begin{array}{l}
-\Delta u+u=(1+a(x)) f(u) \text { em } \mathbb{R}^{N} \\
u \in H^{1}\left(\mathbb{R}^{N}\right)
\end{array}\right.
$$

é estudado com $N \geq 2, f$ uma função assintoticamente linear ou superlinear não necessariamente homogênea e a função peso $a(x)$ tendendo a zero no infinito e podendo mudar de sinal.

Além disso, o sistema do tipo gradiente não homogêneo, assintoticamente linear e fortemente acoplado

$$
\left\{\begin{array}{l}
-\Delta u+u=(1+a(x)) \frac{u\left(u^{2}+v^{2}\right)}{1+s\left(u^{2}+v^{2}\right)}+\lambda v \text { em } \quad \mathbb{R}^{N}, \\
-\Delta v+v=(1+a(x)) \frac{v\left(u^{2}+v^{2}\right)}{1+s\left(u^{2}+v^{2}\right)}+\lambda u \text { em } \quad \mathbb{R}^{N}, \\
u, v \in H^{1}\left(\mathbb{R}^{N}\right),
\end{array}\right.
$$

é estudado com $N \geq 3, \lambda$ e $s$ parâmetros reais satisfazendo $0<s<\frac{1}{1+\lambda}, 0<\lambda<1$ e condições na função peso $a(x)$ semelhantes as do caso escalar.

Usando argumentos topológicos que envolvem uma função baricentro, obtem-se existência de soluções positivas para ambos os problemas em situações em que não existem soluções de energia mínima ground state.

Palavras-Chaves: Equações de Schrödinger; assintoticamente linear; superlinear; solução positiva; métodos variacionais; sistema fortemente acoplado; decaimento exponencial, baricentro. 


\section{Abstract}

The semilinear elliptic problem

$$
\left\{\begin{array}{l}
-\Delta u+u=(1+a(x)) f(u) \text { in } \mathbb{R}^{N}, \\
u \in H^{1}\left(\mathbb{R}^{N}\right)
\end{array}\right.
$$

is studied with $N \geq 2, f$ an asymptotically linear or superlinear not necessarily homogeneous function e the weight function $a(x)$, vanishing at infinity and may change its signal.

In addition, the asymptotically linear and strongly coupled non-homogeneous gradient type system

$$
\left\{\begin{array}{l}
-\Delta u+u=(1+a(x)) \frac{u\left(u^{2}+v^{2}\right)}{1+s\left(u^{2}+v^{2}\right)}+\lambda v \text { in } \mathbb{R}^{N} \\
-\Delta v+v=(1+a(x)) \frac{v\left(u^{2}+v^{2}\right)}{1+s\left(u^{2}+v^{2}\right)}+\lambda u \text { in } \mathbb{R}^{N} \\
u, v \in H^{1}\left(\mathbb{R}^{N}\right)
\end{array}\right.
$$

is studied with $N \geq 3, \lambda$ and $s$ real parameters satisfying $0<s<\frac{1}{1+\lambda}, 0<\lambda<1$ and conditions on the weight function $a(x)$ such as in the scalar case.

Using topological arguments involving a barycenter function, we obtain existence of positive solutions for both problems in situations where there are no ground state solutions.

Key-Words: Schrödinger equations; asymptotically linear; superlinear; positive solution; variational methods; strongly coupled system; exponential decay, barycenter. 


\section{Sumário}

1 Caso escalar $r$

1.1 Preliminares e resultados auxiliares . . . . . . . . . . . . . . . . . . 9

1.2 Compacidade . . . . . . . . . . . . . . . . . . . . . . 19

1.3 Projeção sobre Nehari . . . . . . . . . . . . . . . . . . . . . . . . . 21

1.4 Estimativas assintóticas . . . . . . . . . . . . . . . . . . . 29

1.5 Demonstração do resultado principal . . . . . . . . . . . . . . . . . . . . 46

2 Sistema fortemente acoplado $\quad 50$

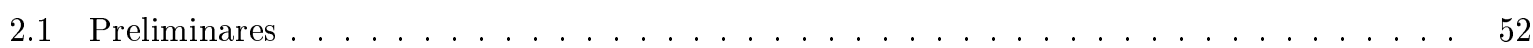

2.2 Decaimento das soluções do problema limite . . . . . . . . . . . . . . . . . 53

2.3 Variedade de Nehari e limitação da sequência de Palais-Smale . . . . . . . . . . . . . 66

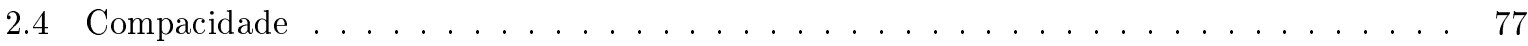

2.5 Estimativas assintóticas . . . . . . . . . . . . . . . . . . 79

2.6 Demonstração do resultado principal do sistema . . . . . . . . . . . . . . . . . 97 


\section{Introdução}

A busca de soluções de campos escalares de equações não lineares usando métodos variacionais tem sido vigoroso nas últimas três décadas, vide [10, 13, 12, 23, 39, 42], entre muitos outros. Neste assunto, equações semilineares elípticas em $\mathbb{R}^{N}$ surgem como ondas estacionárias das equações de Schrödinger e equações de Klein-Gordon surgem na modelagem, por exemplo, da propagação de um feixe de luz em meios Kerr e não-Kerr, como em [3, 44] e suas referências, o que leva ao problema elíptico

$$
(P) \quad\left\{\begin{array}{l}
-\Delta u+V(x) u=f(x, u) \text { em } \mathbb{R}^{N}, \\
u \in H^{1}\left(\mathbb{R}^{N}\right) .
\end{array}\right.
$$

O interesse por este tipo de problema é duplo: por um lado, a vasta gama de aplicações e, por outro lado, o desafio matemático introduzido quando se trabalha em um domínio não limitado como o espaço vetorial $\mathbb{R}^{N}$.

Neste trabalho estamos preocupados especificamente com a seguinte versão simplificada do problema $(P)$ :

$$
\left(P_{a}\right) \quad\left\{\begin{array}{l}
-\Delta u+u=(1+a(x)) f(u) \text { em } \mathbb{R}^{N}, \\
u \in H^{1}\left(\mathbb{R}^{N}\right),
\end{array}\right.
$$

com hipóteses sobre $a(x)$ que implicam este problema não ter uma solução de energia mínima e conduzem ao desafio de procurar soluções em níveis mais elevados de energia. Nossa motivação especial foi o importante trabalho de Bahri e Li [9] onde introduziram um procedimento de min-max para provar a existência de uma solução positiva bound state de

$$
\left(P_{q}\right) \quad\left\{\begin{array}{l}
-\Delta u+u=q(x)|u|^{p-1} u \text { em } \mathbb{R}^{N}, \\
u \in H^{1}\left(\mathbb{R}^{N}\right),
\end{array}\right.
$$

onde $1<p<\frac{N+2}{N-2}$, se $N \geq 3$, e $1<p<+\infty$, se $N=1,2 \operatorname{com} q \in L^{\infty}\left(\mathbb{R}^{N}\right)$ satisfazendo algum limite assintótico exponencial, quando a solução ground state não existe para o problema.

Nosso objetivo é ampliar o artigo [9] para não linearidades $f(u)$ não homogêneas que são superlineares ou assintoticamente lineares no infinito e em que $a(x)$ também satisfaz um limite assintótico exponencial. Nós utilizamos uma abordagem variacional e um argumento topológico introduzido em [9] e atualizado em $[18,24,31]$.

Há uma extensa literatura sobre o assunto. Vamos destacar alguns artigos que são mais relevantes no que diz respeito aos nossos principais objetivos. Nos casos autônomos onde $V(x)=m$ e $f(x, u)=f(u)$, o 
trabalho pioneiro de Berestycki e Lions [13] exibiu uma solução ground state para $(P)$. Usando argumentos de minimização com restrição, eles mostraram a existência de uma solução positiva, radial e investigaram a sua regularidade e o seu decaimento exponencial no infinito. Em 1984, P. L. Lions [27] introduziu idéias inovadoras notáveis de concentração-compacidade que permitiu inúmeras investigações sobre este tipo de problema.

Lehrer e Maia em [29] estudaram o problema $(P)$ com $V(x)=\lambda>0$ e $f(x, u)=a(x) f(u)$ em $\mathbb{R}^{N}$, $f(u)$ assintoticamente linear no infinito e impuseram várias hipóteses sobre $a(x)$. Trabalhando com a conhecida variedade de Pohozaev e usando argumento de Linking, elas provaram a existência de uma solução bound state para o problema.

Clapp e Maia em [18] investigaram a existência de solução ground state positiva para a equação $-\Delta u+V(x) u=f(u)$ in $\mathbb{R}^{N}$ onde $f$ é superlinear ou assintoticamente linear no infinito utilizando técnicas variacionais, no caso em que o nível crítico de energia mínima do problema não é atingido.

Recentemente, Weth e Évéquoz [24] consideraram a equação em $(P)$ com hipóteses sobre $a(x)$, o que os levou a trabalhar com o espaço $H^{1}\left(\mathbb{R}^{N}\right)$ na forma de decomposição espectral $E^{+} \oplus E^{0} \oplus E^{-}$e com $F$, a primitiva de $f$, do tipo superquadrático no infinito. Para ter sucesso na obtenção de estimativas de energia convenientes, eles tiveram que impor outras hipóteses, tais como a existência de uma constante $C>0$ tal que

$$
F(x, u) \geq F_{+\infty}-C e^{-\alpha \sqrt{a_{\infty}}|x|}\left(|u|^{2}+|u|^{p}\right) ; \quad \alpha>0,
$$

e com $f(u)=o\left(|u|^{1+\nu}\right)$, quando $|u| \rightarrow 0$, para algum $\nu>0$. Assumindo essas hipóteses, eles obtiveram uma solução positiva que não é, necessariamente, ground state.

Inspirado pelas idéias em [24] e [31] nós realizamos algumas estimativas precisas de energia e aplicamos um argumento topológico envolvendo a função baricentro para mostrar que existe um valor crítico para o funcional relacionado com a equação de Euler em $\left(P_{a}\right)$, em um nível adequado de energias, dando uma solução para o problema.

Em contraste com as obras mencionadas acima, podemos destacar alguns aspectos relevantes. Distintamente do método em [9], nós evitamos a utilização de uma identidade algébrica (Lema 2.1 em [9]) ao trabalhar com o vínculo da variedade de Nehari e, portanto, permitindo não linearidades $f$ mais gerais que são não homogêneas. Além disso, diferentemente de [29], trabalhamos com a variedade de Nehari em vez da variedade de Pohozaev o que nos permitiu trabalhar com pesos $a(x)$ mais gerais, assim, com menos restrições para o problema. Finalmente, fomos capazes de melhorar as restrições de regularidade da função $f$ exigindo apenas $f \in C^{1}[0,+\infty) \cap C^{2}(0,+\infty)$ explorando alguns cálculos técnicos com a nossa hipótese (ver Observação 1.2 e Lema 1.14 ) e evitando também a utilização do Lema 2.2 em [1] ou as hipóteses $\left(F_{2}^{\prime}\right)$ e $(4)$ em [24].

Segundo o nosso conhecimento, este resultado é novo e examinando as interações de duas cópias de translações da solução positiva ground state do problema limite

$$
\left(P_{\infty}\right) \quad\left\{\begin{array}{l}
-\Delta u+u=f(u) \text { em } \mathbb{R}^{N}, \\
u \in H^{1}\left(\mathbb{R}^{N}\right)
\end{array}\right.
$$

e manipulando estimativas exponenciais delicadas dessas translações, fomos capazes de estender o resultado em [9].

Vamos assumir $N \geq 2$ e também as seguintes hipóteses para a função peso:

$$
\left(a_{1}\right) a \in C\left(\mathbb{R}^{N}\right) ; \inf _{x \in \mathbb{R}^{N}}(1+a(x))=\tau>0 \text { e } \lim _{|x| \rightarrow+\infty} a(x)=0 ;
$$


$\left(a_{2}\right)$ existem constantes $p_{1}, p_{2}$ com $1<p_{1} \leq p_{2}<2^{*}-1$ tais que $|a(x)| \leq C_{1} e^{-k|x|}$ onde $k \in\left(2, p_{1}+1\right)$, para todo $x \in \mathbb{R}^{N}$ e $C_{1}$ uma constante positiva.

Ademais, nós vamos considerar $f$, não necessariamente homogênea, satisfazendo:

$\left(f_{1}\right) f \in C^{1}[0,+\infty) \cap C^{2}(0,+\infty)$

$\left(f_{2}\right) f$ e sua derivada de primeira ordem têm o seguinte crescimento

$$
\left|f^{(k)}(t)\right| \leq C\left(|t|^{p_{1}-k}+|t|^{p_{2}-k}\right),
$$

para $k \in\{0,1\}$ para $t>0$ e $C>0$ uma constante;

$\left(f_{3}\right) f^{\prime}(t)>\frac{f(t)}{t}$, se $t>0$

$\left(f_{4}\right) \lim _{t \rightarrow+\infty} \frac{f(t)}{t} \geq l_{\infty}>1$ para algum $l_{\infty} \in \mathbb{R}$

$\left(f_{5}\right) \lim _{t \rightarrow+\infty}\{f(t) t-2 F(t)\}=+\infty$ onde $F(t):=\int_{0}^{t} f(\varsigma) d \varsigma ;$

$(U)$ A solução positiva do problema $\left(P_{\infty}\right)$ é única.

Nosso primeiro objetivo neste trabalho é tentar demonstrar o seguinte resultado:

Teorema 0.1. Assumindo que as hipóteses $\left(a_{1}\right)-\left(a_{2}\right),\left(f_{1}\right)-\left(f_{5}\right)$ e $(U)$ são satisfeitas, então o problema $\left(P_{a}\right)$ tem uma solução positiva $u \in H^{1}\left(\mathbb{R}^{N}\right)$. Além disso, se a $\in C_{\text {loc }}^{1, \alpha}\left(\mathbb{R}^{N}\right)$, então a solução é clássica.

A hipótese de unicidade de solução positiva de $\left(P_{\infty}\right)$ é fundamental para o método construtivo que será aplicado na obtenção de solução positiva de $(P)$. Tal unicidade é conhecida no caso da pôtencia pura superlinear e subcrítica $f(s)=|s|^{p}$, com $1<p<2^{*}-1$ demonstrado por Kwong em [26], e no caso da não linearidade modelo, assintoticamente linear, $f(s)=l_{\infty} \frac{s^{3}}{1+s^{2}}$ demonstrado em Serrin e Tang [40]. Estes dois exemplos de não linearidades $f$ satisfazem as nossas hipóteses $\left(f_{1}\right)-\left(f_{5}\right)$. Porém, em geral a unicidade pode não ocorrer. Resultados de condição suficiente podem ser encontrados em [36] e [40]. Por exemplo, se a função $h(u):=\frac{-u+f(u)}{u f^{\prime}(u)-f(u)}$ é não decrescente em $(\tau, \infty)$ onde $\tau$ é o único número positivo satisfazendo $\frac{f(\tau)}{\tau}=1$, então tem-se uma condição suficiente para a unicidade da solução positiva de $\left.P_{\infty}\right)$.

A hipótese $\left(f_{4}\right)$ complementa $\left(f_{3}\right)$ afirmando que $\lim _{t \rightarrow+\infty} \frac{f(t)}{t}$ pode ser $+\infty$ ou uma constante $l_{\infty}$. Neste segundo caso, a constante $l_{\infty}$ deve ser maior que 1 para que $\left(P_{\infty}\right)$ tenha solução não trivial segundo Berestycki e Lions [13] com $m=1$.

Em um segundo momento, sobre o estudo de sistemas, podemos citar o clássico trabalho de AmbrosettiCerami - Ruiz [5]. Usando argumentos de concentração de compacidade os autores mostraram resultados de existência de solução positiva do tipo ground e bound state do seguinte sistema

$$
\left\{\begin{array}{rlll}
-\Delta u+u & =(1+a(x))|u|^{p-1} u+\lambda v & \text { em } & \mathbb{R}^{N} \\
-\Delta v+v & =(1+b(x))|v|^{p-1} v+\lambda u & \text { em } & \mathbb{R}^{N}
\end{array}\right.
$$


considerando $N \geq 2,(u, v) \in W^{1,2}\left(\mathbb{R}^{N}\right) \times W^{1,2}\left(\mathbb{R}^{N}\right), 0<\lambda<1$ um parâmetro real, $\lim _{|x| \rightarrow+\infty} a(x)=0=$ $\lim _{|x| \rightarrow+\infty} b(x)$ e, ainda $\inf _{x \in \mathbb{R}^{N}}(1+a(x))>0$ e $\inf _{x \in \mathbb{R}^{N}}(1+b(x))>0$, além de assumirem normas $|a|_{L^{\infty}}$ e $|b|_{L^{\infty}}$ suficientemente pequenas.

Ambrosetti [4], usando métodos de perturbação, provou a existência de soluções para o sistema não autônomo não linear de equações de Schrödinger as quais são linearmente acopladas

$$
\left\{\begin{array}{rlll}
-u_{1}^{\prime \prime}+u_{1} & =\left(1+\varepsilon b_{1}(x)\right) u_{1}^{3}+\gamma u_{2} & \text { em } & \mathbb{R} \\
-u_{2}^{\prime \prime}+u_{2} & =\left(1+\varepsilon b_{2}(x)\right) u_{2}^{3}+\gamma u_{1} & \text { em } & \mathbb{R}
\end{array}\right.
$$

onde $\gamma$ e $\varepsilon$ são parâmetros reais, $b_{i} \in L^{\infty}(\mathbb{R}), \lim _{|x| \rightarrow+\infty} b_{i}(x)=0 ; u_{1}, u_{2} \in W^{1,2}(\mathbb{R}) \operatorname{com} i=1,2$. Quando $\varepsilon=0$, este sistema foi estudado em [3] do ponto de vista analítico e numérico e quando $b_{i} \equiv 0$ e $\varepsilon=1$, o sistema foi estudado por [6] mostrando a existência de solução com componente multi-bump.

Em 2013, Zhang, Xu e Zhang em [48] estudaram o sistema

$$
\left\{\begin{array}{llll}
-\Delta u+(1+a(x)) u & =F_{u}(u, v)+\lambda v & \text { em } & \mathbb{R}^{N} \\
-\Delta v+(1+b(x)) v & =F_{v}(u, v)+\lambda u & \text { em } & \mathbb{R}^{N}
\end{array}\right.
$$

para $N \geq 2, a, b$ periódicos ou assintoticamente periódicos e $F$ superlinear. A ferramenta utilizada para mostrar a existência de ground state positiva foi a variedade de Nehari e princípio de concentração de compacidade.

Lehrer e Maia em [29] estudaram o problema $-\Delta u+\lambda u=a(x) f(u)$ em $\mathbb{R}^{N}$, com $f$ assintoticamente linear no infinito e impondo várias hipóteses sobre $a(x)$. Usando a variedade de Pohozaev e argumentos de Linking, as autoras provaram a existência de solução bound state para o problema. Com as devidas adaptações, Lehrer e Maia também garantiram a existência de solução não nula via Teorema de Linking para o sistema

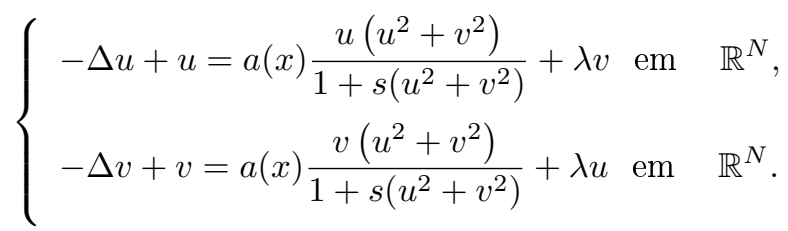

Apesar de no caso modelo o termo não linear $f$, assintoticamente linear, satisfazer a hipótese $f(t) / t$ crescente para $t>0$, ou no caso do sistema $\nabla F(t u, t v)(u, v) / t$ crescente na variável $t$, para $(u, v) \neq(0,0) \mathrm{e}$ permitir a projeção sobre a variedade de Nehari do funcional e a obtenção de pontos críticos dos funcionais associados sob este vínculo, este aspecto não foi utilizado em [29] permitindo-se não linearidades mais gerais, sem esta característica. Assim sendo, tanto no caso escalar quanto no caso do sistema foram usadas projeções sob a variedade de Pohozaev do funcional. Entretanto para tratar os termos não lineares não homogẽneos com hipóteses de crescimento menos restritivas, foram necessárias hipóteses extras de regularidade e crescimento sobre o peso $a(x)$.

Existe uma motivação Física para o estudo deste tipo de sistema de duas equações de Schrödinger acopladas, nas quais encontra-se um acoplamento linear nas duas componentes. Um exemplo é o sistema de duas equações que modelam os condensados de Bose-Einstein compostos por dois estados hiperfinos (Ver [11] e suas referências). Tais modelos consideram um grande número de pequenos componentes individuais que interagem uns com os outros; o fenômeno é descrito pelas equações de Gross-Pitaevskii em $[34,35]$ 


$$
\left\{\begin{array}{l}
i \frac{\partial \psi_{1}}{\partial t}=\left(L_{1}+U_{11}\left|\psi_{1}\right|^{2}+U_{12}\left|\psi_{2}\right|^{2}\right) \psi_{1}+\lambda \psi_{2} \\
i \frac{\partial \psi_{2}}{\partial t}=\left(L_{2}+U_{22}\left|\psi_{2}\right|^{2}+U_{21}\left|\psi_{2}\right|^{2}\right) \psi_{2}+\lambda \psi_{1}
\end{array}\right.
$$

onde $L_{j}=-\partial^{2} / \partial x^{2}+V_{j} \operatorname{com} j=1,2$. Embora a dinâmica dos condensados acoplados de Bose-Einstein tenha atraído a atenção nos últimos anos, o problema acima também surge em outros contextos físicos, mais especificamente em modelos ópticos não-lineares em que as equações descrevem feixes de luz em fibras ópticas onde $\psi_{1}$ e $\psi_{2}$ representam o campo de luz dentro de ondas eletromagnéticas ou ondas sonoras em [47]. Os sistemas elípticos encontrados em [5, 29] podem ser obtidos de (1) quando se estuda a existência de soluções estacionárias (solitárias) para o sistema, isto é, uma solução da forma $\psi_{1}(x, t)=\exp (-i E t) u(x)$ e $\psi_{2}(x, t)=\exp (-i E t) v(x)$.

Inspirados pelos trabalhos de Ambrosetti - Cerami - Ruiz em [5], Clapp - Maia em [18] e Lehrer Maia em [29], nos propusemos a estudar o seguinte sistema fortemente acoplado com não-linearidade assintoticamente linear no infinito

$$
(\mathbb{S})\left\{\begin{array}{l}
-\Delta u+u=(1+a(x)) \frac{u\left(u^{2}+v^{2}\right)}{1+s\left(u^{2}+v^{2}\right)}+\lambda v \text { em } \quad \mathbb{R}^{N}, \\
-\Delta v+v=(1+a(x)) \frac{v\left(u^{2}+v^{2}\right)}{1+s\left(u^{2}+v^{2}\right)}+\lambda u \text { em } \quad \mathbb{R}^{N}, \\
u, v \in H^{1}\left(\mathbb{R}^{N}\right),
\end{array}\right.
$$

com $N \geq 3,0<\lambda<1$ e $s$ um parâmetro real satisfazendo $0<s<\frac{1}{1+\lambda}$. Baseados no método de Bahri - Li em [9] realizamos estimativas de energia mais precisas e aplicamos um argumento topológico envolvendo a função baricentro para mostrar que existe um valor crítico para o funcional relacionado com a equação de Euler do sistema $(\mathbb{S})$, em um nível adequado de energias, dando uma solução para o sistema.

Comparando-se com as obras mencionadas acima, podemos destacar alguns aspectos relevantes. Distintamente do método em [5] que trata do problema homogêneo, utilizamos técnicas de combinação convexa de duas cópias da solução ground state transladadas, técnica introduzida por Bahri-Li em [9], para tratarmos do nosso problema não homogêneo. Além disso, diferentemente de [5] que admite $|a|_{L^{\infty}} \mathrm{e}$ $|b|_{L^{\infty}}$ suficientemente pequenas, estamos livres para não assumir esta restrição nas normas do supremos, porém assumir um decaimento exponencial para $a(x)$, visto que $a(x)$ é positivo e/ou negativo (isto é, podendo mudar de sinal). Complementando os resultados em [29], trabalhamos com a variedade de Nehari em vez da variedade de Pohozaev o que nos permitiu trabalhar com funções pesos $a(x)$ contínuas e assim mais gerais do que os pesos de classe $C^{2}$ encontrados em [29]. Por fim, destacamos a necessidade de demonstrar o decaimento exponencial da solução do problema limite relacionado ao sistema ( $\mathbb{S}$ ) na Seção 2.2 , visto que o resultado provavelmente existe na literatura, entretanto não encontramos uma referência.

Vamos assumir as seguintes hipóteses sobre a função peso para o sistema $(\mathbb{S})$

$\left(a_{1}\right) a \in C\left(\mathbb{R}^{N}\right) ; \inf _{x \in \mathbb{R}^{N}}(1+a(x))=\varsigma>0$ e $\lim _{|x| \rightarrow+\infty} a(x)=0$

$\left(a_{2}\right)|a(x)| \leq C e^{-k|x|}$ onde $k \in(2 \sqrt{1-\lambda}, 4 \sqrt{1-\lambda})$ para todo $x \in \mathbb{R}^{N}$ e $C$ uma constante positiva; 
$(U)$ A solução positiva $(u, v), u>0, v>0$ do sistema limite

$$
\left(\mathbb{S}_{\infty}\right)\left\{\begin{array}{l}
-\Delta u+u=\frac{u\left(u^{2}+v^{2}\right)}{1+s\left(u^{2}+v^{2}\right)}+\lambda v, \quad \mathbb{R}^{N}, \\
-\Delta v+v=\frac{v\left(u^{2}+v^{2}\right)}{1+s\left(u^{2}+v^{2}\right)}+\lambda u, \quad \mathbb{R}^{N}, \\
u, v \in H^{1}\left(\mathbb{R}^{N}\right) .
\end{array}\right.
$$

é única ou qualquer solução positiva está no mesmo nível de energia do funcional associado a $\left(\mathbb{S}_{\infty}\right)$.

O nosso principal resultado sobre sistemas é o seguinte teorema

Teorema 0.2. Assuma $0<\lambda<1,0<s<\frac{1}{1+\lambda}$, as hipóteses $\left(a_{1}\right),\left(a_{2}\right)$ e $(U)$, então o problema $(\mathbb{S})$ tem uma solução positiva $(u, v) \in H^{1}\left(\mathbb{R}^{N}\right) \times H^{1}\left(\mathbb{R}^{N}\right)$. Além disso, se a $\in C_{\text {loc }}^{1, \alpha}\left(\mathbb{R}^{N}\right)$, então a solução $(u, v)$ é clássica.

Este trabalho está estruturado da seguinte forma: no Capítulo 1, estudaremos o problema escalar

$$
\left\{\begin{array}{l}
-\Delta u+u=(1+a(x)) f(u) \text { em } \mathbb{R}^{N}, \\
u \in H^{1}\left(\mathbb{R}^{N}\right),
\end{array}\right.
$$

realizando algumas estimativas de energia e aplicando um argumento topológico envolvendo a função baricentro para mostrar que existe um valor crítico para o funcional relacionado com a equação de Euler do problema acima, em um nível adequado de energias, dando uma solução para o problema. Especificamente, na Seção 1.1 trataremos de alguns resultados auxiliares; na Seção 1.2 estudaremos a compacidade da sequência de Palais-Smale para o funcional associado; na Seção 1.3 demonstraremos a projeção sobre a variedade de Nehari; na Seção 1.4 realizaremos as estimativas de energia assintótica do funcional associado e decaimento exponecial das soluções do problema limite, por fim, na Seção 1.5 apresentaremos a prova do resultado principal deste capítulo utilizando argumentos topológicos.

No Capítulo 2, estudaremos o sistema do tipo gradiente não homogêneo, fortemente acoplado

$$
\left\{\begin{array}{l}
-\Delta u+u=(1+a(x)) \frac{u\left(u^{2}+v^{2}\right)}{1+s\left(u^{2}+v^{2}\right)}+\lambda v \text { em } \mathbb{R}^{N}, \\
-\Delta v+v=(1+a(x)) \frac{v\left(u^{2}+v^{2}\right)}{1+s\left(u^{2}+v^{2}\right)}+\lambda u \text { em } \mathbb{R}^{N}, \\
u, v \in H^{1}\left(\mathbb{R}^{N}\right),
\end{array}\right.
$$

com $0<\lambda<1$ e $s$ é um parâmetro real satisfazendo $0<s<\frac{1}{1+\lambda}$. Mostraremos primeiramente o decaimento exponencial da solução do problema limite na Seção 2.2 e, obteremos uma solução positiva para o sistema seguindo a técnica utilizada no caso escalar e em Clapp - Maia [18]. Especificamente, na Seção 2.1 trataremos de alguns resultados preliminares; na Seção 2.2 estudaremos o decaimento exponecial das soluções do problema limite; na Seção 2.3 estudaremos a variedade de Nehari e provaremos a limitação da sequência de Palais-Smale; na Seção 2.4 estudaremos a compacidade da sequência de Palais-Smale para o funcional associado; na Seção 2.5 estudaremos as estimativas assintóticas do funcional associado; por fim, na Seção 2.6 apresentaremos a prova do resultado principal deste capítulo. 


\section{Capítulo}

1

\section{Caso escalar}

Neste capítulo, como descrito na introdução, estudaremos o problema

$$
\left(P_{a}\right) \quad\left\{\begin{array}{l}
-\Delta u+u=(1+a(x)) f(u) \text { em } \mathbb{R}^{N}, \\
u \in H^{1}\left(\mathbb{R}^{N}\right) .
\end{array}\right.
$$

Vamos assumir $N \geq 2$ e também as seguintes hipóteses para a função peso:

$\left(a_{1}\right) a \in C\left(\mathbb{R}^{N}\right) ; \inf _{x \in \mathbb{R}^{N}}(1+a(x))=\tau>0 \quad$ e $\quad \lim _{|x| \rightarrow+\infty} a(x)=0$

$\left(a_{2}\right)$ existem constantes $p_{1}, p_{2}$ com $1<p_{1} \leq p_{2}<2^{*}-1$ tais que $|a(x)| \leq C_{1} e^{-k|x|}$ onde $k \in\left(2, p_{1}+1\right)$, para todo $x \in \mathbb{R}^{N}$ e $C_{1}$ uma constante positiva.

Outrossim, vamos considerar $f$ não necessariamente homogênea satisfazendo

$\left(f_{1}\right) f \in C^{1}[0,+\infty) \cap C^{2}(0,+\infty)$;

$\left(f_{2}\right) f$ e sua derivada de primeira ordem têm o seguinte crescimento

$$
\left|f^{(k)}(t)\right| \leq C\left(|t|^{p_{1}-k}+|t|^{p_{2}-k}\right),
$$

para $k \in\{0,1\}$ para $t>0$ e onde $C>0$ é uma constante;

$\left(f_{3}\right) f^{\prime}(t)>\frac{f(t)}{t}$, se $t>0$

$\left(f_{4}\right) \lim _{t \rightarrow+\infty} \frac{f(t)}{t} \geq l_{\infty}>1$ para algum $l_{\infty} \in \mathbb{R} ;$

$\left(f_{5}\right) \lim _{t \rightarrow+\infty}\{f(t) t-2 F(t)\}=+\infty$, em que $F(t):=\int_{0}^{t} f(\varsigma) \mathrm{d} \varsigma ;$

$(U)$ A solução positiva do problema $\left(P_{\infty}\right)$ é única.

Observação 1.1. Note que a hipótese $\left(f_{3}\right)$ implica que

$$
\frac{1}{2} f(t) t-F(t)>0, \text { se } t \neq 0
$$


Esta implicação junto com a hipótese $\left(f_{5}\right)$ é conhecido por condição de não quadraticidade de $f$.

Observação 1.2. Note também que a hipótese $\left(f_{2}\right)$ implica que, para toda constante positiva $\mu$ satisfazendo $0<1+\mu<p_{1}$, tem-se $f(u)=o\left(|u|^{1+\mu}\right)$, quando $|u| \rightarrow 0$.

Como o problema é variacional, nós precisamos introduzir ferramentas variacionais para podermos demonstrar os resultados subsequentes. Inicialmente vamos definir $f(t):=-f(-t)$ para $t<0$. Assim temos que $f$ é de classe $C^{2}(\mathbb{R})$ e é uma função ímpar. É importante observar que se $u$ é solução positiva do problema $\left(P_{a}\right)$ para essa nova função, $u$ é também solução do problema $\left(P_{a}\right)$ para a função original. Vamos, então, considerar esta extensão e estabelecer a existência de solução positiva para o problema.

Iremos usar a constante geral $C>0$ para simplificar a notação e assim $C$ não será sempre a mesma constante no que segue. Considerando o espaço de Hilbert $H^{1}\left(\mathbb{R}^{N}\right)$ com o produto interno definido por

$$
\langle u, v\rangle:=\int_{R^{N}}(\nabla u \nabla v+u v) \mathrm{d} x
$$

e a norma associada

$$
\|u\|^{2}:=\int_{R^{N}}\left(|\nabla u|^{2}+u^{2}\right) \mathrm{d} x .
$$

O funcional associado ao problema $\left(P_{a}\right)$ é definido por

$$
I(u):=\frac{1}{2}\|u\|^{2}-\int_{R^{N}}(1+a(x)) F(u) \mathrm{d} x
$$

e a sua derivada

$$
I^{\prime}(u) v=\langle u, v\rangle-\int_{R^{N}}(1+a(x)) f(u) v \mathrm{~d} x .
$$

Trabalharemos com a variedade de Nehari definida por

$$
\begin{aligned}
\mathcal{N} & :=\left\{u \in H^{1}\left(\mathbb{R}^{N}\right) \backslash\{0\} ; J(u)=0\right\} \\
& =\left\{u \in H^{1}\left(\mathbb{R}^{N}\right) \backslash\{0\} ;\|u\|^{2}=\int_{R^{N}}(1+a(x)) f(u) u \mathrm{~d} x\right\}
\end{aligned}
$$

cujo funcional associado é

$$
J(u):=I^{\prime}(u) u=\|u\|^{2}-\int_{R^{N}}(1+a(x)) f(u) u \mathrm{~d} x .
$$

Consideremos também o nível de energia mínima associado ao funcional $I$ definido por

$$
m:=\inf _{u \in \mathcal{N}} I(u)
$$

Associado ao problema $\left(P_{a}\right)$, por $\left(a_{1}\right)$, utilizaremos fortemente o problema limite

$$
\left(P_{\infty}\right) \quad\left\{\begin{array}{r}
-\Delta u+u=f(u) \text { em } \mathbb{R}^{N}, \\
u \in H^{1}\left(\mathbb{R}^{N}\right)
\end{array}\right.
$$

e, associado a este problema limite, definimos o nível de energia mínima:

$$
m_{\infty}:=\inf _{u \in \mathcal{N}_{\infty}} I_{\infty}(u)
$$


para o funcional associado definido por

$$
I_{\infty}(u):=\frac{1}{2}\|u\|^{2}-\int_{R^{N}} F(u) \mathrm{d} x
$$

e sua derivada dada por

$$
I_{\infty}^{\prime}(u) v=\langle u, v\rangle-\int_{R^{N}} f(u) v \mathrm{~d} x, \quad v \in H^{1}\left(\mathbb{R}^{N}\right) .
$$

Analogamente, o funcional associado a $\mathcal{N}_{\infty}$ é definido por

$$
J_{\infty}(u):=I_{\infty}^{\prime}(u) u=\|u\|^{2}-\int_{R^{N}} f(u) u \mathrm{~d} x
$$

\subsection{Preliminares e resultados auxiliares}

Feitas estas considerações iniciais, nós pretendemos demonstrar o primeiro resultado que contém as propriedades principais sobre a variedade $\mathcal{N}$ associada ao funcional $I$.

Lema 1.1. A variedade $\mathcal{N}$ satisfaz:

a) existe um número $\alpha>0$ tal que para todo $u \in \mathcal{N}$ tem-se $\|u\| \geq \alpha$;

b) $\mathcal{N}$ é uma subvariedade fechada, de classe $C^{2}$ em $H^{1}\left(\mathbb{R}^{N}\right)$ e é variedade natural para $I$;

c) para todo $u \in \mathcal{N}$, a função $t \mapsto g(t):=I(t u)$ é estritamente crescente no intervalo $[0,1)$ e estritamente decrescente no intervalo $(1,+\infty)$. Assim, em particular, pode-se afirmar que

$$
I(u)=\max _{t>0} I(t u)>0
$$

Demonstração: Verificação de $(a)$ Por $\left(a_{1}\right)$ temos $a \in C\left(\mathbb{R}^{N}\right) \cap L^{\infty}\left(\mathbb{R}^{N}\right)$. Segue que $1+\|a(x)\|_{\infty} \leq C$. Usando a expressão de $J$ em $(1.5),\left(f_{2}\right)$, para todo $u \in \mathcal{N}$ obtemos

$$
\begin{aligned}
J(u) & =I^{\prime}(u) u=\|u\|^{2}-\int_{\mathbb{R}^{N}}(1+a(x)) f(u) u \mathrm{~d} x \\
& \geq\|u\|^{2}-C \int_{\mathbb{R}^{N}}\left(|u|^{p_{1}+1}+|u|^{p_{2}+1}\right) \mathrm{d} x .
\end{aligned}
$$

Note que $2<p_{1}+1 \leq p_{2}+1$. Logo, existe um $t \in(0,1)$ que nos permite escrever $p_{1}+1=$ $2 t+(1-t)\left(p_{2}+1\right)$. Daí, pela desigualdade de Hölder segue que

$$
\int_{\mathbb{R}^{N}}|u|^{p_{1}+1} \mathrm{~d} x=\int_{\mathbb{R}^{N}}|u|^{2 t}|u|^{(1-t)\left(p_{2}+1\right)} \mathrm{d} x \leq\left(\int_{\mathbb{R}^{N}}|u|^{2} \mathrm{~d} x\right)^{t}\left(\int_{R^{N}}|u|^{p_{2}+1} \mathrm{~d} x\right)^{1-t}
$$

$\operatorname{com} p=\frac{1}{t}$ e $p^{\prime}=\frac{1}{1-t}$. Assim,

$$
\int_{\mathbb{R}^{N}}|u|^{p_{1}+1} \mathrm{~d} x \leq\|u\|_{L^{2}\left(\mathbb{R}^{N}\right)}^{2 t}\|u\|_{L^{p_{2}+1}\left(\mathbb{R}^{N}\right)}^{\left(p_{2}+1\right)(1-t)}
$$

Desde que temos a imersão contínua $H^{1}\left(\mathbb{R}^{N}\right) \hookrightarrow L^{q}\left(\mathbb{R}^{N}\right)$ para $2 \leq q \leq 2^{*}$ e temos $p_{2}+1<2^{*}$ obtemos 


$$
\int_{\mathbb{R}^{N}}|u|^{p_{1}+1} \mathrm{~d} x \leq\|u\|_{H^{1}}^{2 t}\|u\|_{H^{1}}^{\left(p_{2}+1\right)(1-t)}
$$

Sabendo, pela desigualdade de Young que $a b \leq \frac{1}{p} a^{p}+\frac{1}{p^{\prime}} b^{p^{\prime}}=t a^{1 / t}+(1-t) b^{1 /(1-t)}$, podemos reescrever a expressão anterior da seguinte forma

$$
\begin{aligned}
\int_{\mathbb{R}^{N}}|u|^{p_{1}+1} \mathrm{~d} x & \leq \frac{1}{C}\|u\|_{H^{1}}^{2 t} C\|u\|_{H^{1}}^{\left(p_{2}+1\right)(1-t)} \\
& \leq \frac{t}{C}\|u\|_{H^{1}}^{2}+(1-t)(C)^{1 /(1-t)}\|u\|_{H^{1}}^{p_{2}+1} .
\end{aligned}
$$

Usando a imersão contínua novamente temos que

$$
\int_{\mathbb{R}^{N}}|u|^{p_{2}+1} \mathrm{~d} x=C\|u\|_{L^{p_{2}+1}\left(\mathbb{R}^{N}\right)}^{p^{2}+1} \leq C\|u\|_{H^{1}}^{p_{2}+1} .
$$

Usando as estimativas (1.12) e (1.13) na expressão de $J$ em (1.11) obtemos

$$
\begin{aligned}
J(u) & \geq\|u\|^{2}-t\|u\|^{2}-C\|u\|^{p_{2}+1} \\
& =(1-t)\|u\|^{2}-C\|u\|^{p_{2}+1} .
\end{aligned}
$$

Por fim, se $u \in \mathcal{N}$ temos $J(u)=0$. Segue que

$$
\|u\|^{p_{2}-1}=C \frac{\|u\|^{p_{2}+1}}{\|u\|^{2}} \geq \frac{1-t}{C} .
$$

Portanto, existe $\alpha>0$ tal que $\|u\| \geq \alpha, \forall u \in \mathcal{N}$.

Verificação de $(b)$ Note que $\mathcal{N}:=\left\{u \in H^{1}\left(\mathbb{R}^{N}\right) \backslash\{0\} ; J(u)=0\right\}=J^{-1}(\{0\})$. Como $J$ é contínuo, segue que $\mathcal{N}$ é uma subvariedade fechada de $H^{1}\left(\mathbb{R}^{N}\right)$.

Além disso, derivando-se o funcional $J$ em (1.5) e aplicando em $v \in H^{1}\left(\mathbb{R}^{N}\right)$,

$$
J^{\prime}(u) v=2\langle u, v\rangle-\int_{\mathbb{R}^{N}}(1+a(x))\left[f^{\prime}(u) u+f(u)\right] v \mathrm{~d} x .
$$

Em particular, tomando-se $v=u$, segue que

$$
\begin{aligned}
J^{\prime}(u) u & =2\langle u, u\rangle-\int_{\mathbb{R}^{N}}(1+a(x))\left[f^{\prime}(u) u+f(u)\right] u \mathrm{~d} x \\
& =2\|u\|^{2}-\int_{\mathbb{R}^{N}}(1+a(x))\left[f^{\prime}(u) u+f(u)\right] u \mathrm{~d} x .
\end{aligned}
$$

Se $u \in \mathcal{N}$, então $J(u)=0$, isto é, $\|u\|^{2}=\int_{\mathbb{R}^{N}}(1+a(x)) f(u) u \mathrm{~d} x$. 
Substituindo na expressão (1.14) temos por $\left(a_{1}\right)$ e $\left(f_{3}\right)$

$$
\begin{aligned}
J^{\prime}(u) u= & 2 \int_{\mathbb{R}^{N}}(1+a(x)) f(u) u \mathrm{~d} x \\
& -\int_{\mathbb{R}^{N}}(1+a(x))\left[f^{\prime}(u) u+f(u)\right] u \mathrm{~d} x \\
= & \int_{\mathbb{R}^{N}}(1+a(x))\left[-f^{\prime}(u) u^{2}+f(u) u\right] \mathrm{d} x \\
= & \int_{\mathbb{R}^{N}}(1+a(x))\left[-f^{\prime}(u) u+f(u)\right] u \cdot \frac{u}{u} \mathrm{~d} x \\
= & \int_{\mathbb{R}^{N}}(1+a(x))\left[\frac{f(u)-f^{\prime}(u) u}{u}\right] u^{2} \mathrm{~d} x<0 .
\end{aligned}
$$

De fato, se a condição $\left(f_{3}\right)$ é verdade, seja $u>0$, então $\frac{-f(u)+f^{\prime}(u) u}{u}>0$. Isto significa que se $u>0$, então $\frac{f(u)}{u}$ é crescente. Agora, se considerarmos $u<0$, por $\left(f_{3}\right)$, segue que $\frac{f(u)}{u}$ é descrescente. Para verificar isto, note que

$$
\left(\frac{f(u)}{u}\right)^{\prime}=\frac{f^{\prime}(u) u-f(u)}{u^{2}} .
$$

Como $f$ é ímpar, sua derivada será par. Daí, fazendo $v=-u>0$ segue que

$$
\begin{aligned}
\left(\frac{f(u)}{u}\right)^{\prime} & =\frac{f^{\prime}(-v)(-v)-f(-v)}{-v} \frac{1}{-v} \\
& =\frac{f^{\prime}(v)(-v)+f(v)}{v} \frac{1}{-v} \\
& =\frac{f^{\prime}(v)(v)-f(v)}{v} \frac{1}{-v}<0 .
\end{aligned}
$$

Segue que $f^{\prime}(u) u-f(u)<0$, caso $u<0$. Tomando a integral em (1.15) nos conjuntos $\{u>0\}$ e $\{u<0\}$ para $u$ em $\mathcal{N}$ segue que

$$
J^{\prime}(u) u<0
$$

Isto implica que 0 é um valor regular de $J: H^{1}\left(\mathbb{R}^{N}\right) \backslash\{0\} \rightarrow \mathbb{R}$. Assim, $J^{-1}(\{0\})$ é um conjunto fechado, por ser a imagem inversa de um conjunto fechado por um funcional contínuo. Como $\{u \equiv 0\}$ é um ponto isolado de $J^{-1}(\{0\})$, então temos que $\mathcal{N}$ é de classe $C^{2}$ e é uma variedade natural para $I$.

Verificação de $(c)$ Pelo item $(a)$ existe um $\alpha>0$ tal que $\|u\| \geq \alpha$ e, portanto, que $u$ não é identicamente zero. Defina os seguintes conjuntos:

$$
\Gamma^{+}=\left\{x \in \mathbb{R}^{N}: u(x)>0\right\}
$$

e

$$
\Gamma^{-}=\left\{x \in \mathbb{R}^{N}: u(x)<0\right\} .
$$

Como o funcional associado ao problema $\left(P_{a}\right)$ é definido por

$$
I(u):=\frac{1}{2}\|u\|^{2}-\int_{R^{N}}(1+a(x)) F(u) \mathrm{d} x,
$$

considere $t>0$ e $u$ fixado, definimos 


$$
g(t):=I(t u)=\frac{t^{2}}{2}\|u\|^{2}-\int_{R^{N}}(1+a(x)) F(t u) \mathrm{d} x .
$$

Derivando $g$ em relação a $t$ e usando os conjuntos $\Gamma^{+}, \Gamma^{-}$definidos anteriormente, segue que

$$
\begin{aligned}
g^{\prime}(t) & =t\|u\|^{2}-\int_{R^{N}}(1+a(x)) f(t u) u \mathrm{~d} x \\
& =t\|u\|^{2}-\frac{t}{t} \int_{R^{N}}(1+a(x)) f(t u) u \mathrm{~d} x \\
& =t\left\{\|u\|^{2}-\int_{R^{N}}(1+a(x)) \frac{f(t u)}{t} u \mathrm{~d} x\right\} \\
& =t\left\{\int_{R^{N}}(1+a(x)) f(u) u \mathrm{~d} x-\int_{R^{N}}(1+a(x)) \frac{f(t u)}{t} u \mathrm{~d} x\right\} \\
& =t\left\{\int_{R^{N}}(1+a(x))\left(\frac{f(u)}{u}-\frac{f(t u)}{t u}\right) u^{2} \mathrm{~d} x\right\},
\end{aligned}
$$

e reescrevendo $g^{\prime}(t)$

$$
g^{\prime}(t)=t\left\{\int_{\Gamma^{+}}(1+a(x))\left(\frac{f(u)}{u}-\frac{f(t u)}{t u}\right) u^{2} \mathrm{~d} x\right\}+t\left\{\int_{\Gamma^{-}}(1+a(x))\left(\frac{f(u)}{u}-\frac{f(t u)}{t u}\right) u^{2} \mathrm{~d} x\right\} .
$$

Agora observe que se $t \in(0,1)$ e $u \in(0,+\infty)$ temos $t u<u$. Pela hipótese $\left(f_{3}\right)$ temos $\frac{f(t u)}{t u}<\frac{f(u)}{u}$. Além disso por $\left(a_{1}\right)$ segue que $(1+a(x))>0, \forall x \in \mathbb{R}^{N}$ e resulta que $g^{\prime}(t)>0$, isto é, $g$ é estritamente crescente. Se $t \in(0,1)$ e $u \in(-\infty, 0)$ temos $u<t u<0$ e assim

$$
\frac{f(t u)}{t u}=-\frac{f(t(-u))}{t u}=\frac{f(t(-u))}{t(-u)}<\frac{f(-u)}{-u}=\frac{f(u)}{u}
$$

analogamente segue que $g^{\prime}(t)>0$. De maneira análoga, se $t \in(1,+\infty)$ e $u \in(-\infty, 0)$ temos $u>t u$. Pela hipótese $\left(f_{3}\right)$ temos $\frac{f(t u)}{t u}>\frac{f(u)}{u}$. Além disso, por $\left(a_{1}\right)$, segue que $(1+a(x))>0, \forall x \in \mathbb{R}^{N}$ e resulta que $g^{\prime}(t)<0$, isto é, $g$ é estritamente decrescente. Analogamente, se $t \in(1,+\infty)$ e $u \in(0,+\infty)$. Portanto, temos para todo $u \in \mathcal{N}$ que

$$
g(1):=I(u)=\max _{t>0} g(t)=\max _{t>0} I(t u)
$$

O próximo resultado nos dá uma limitação para uma sequência em $\mathcal{N}$ em qualquer nível $d$ fixado. Isto nos permitirá usar o Lema de Splitting posteriormente.

Lema 1.2. Suponha que exista uma sequência $\left\{u_{n}\right\}$ em $\mathcal{N}$ satisfazendo

$$
I\left(u_{n}\right) \rightarrow d
$$

Então a sequência $\left\{u_{n}\right\}$ é limitada em $H^{1}\left(\mathbb{R}^{N}\right)$.

Demonstração: Primeiramente fixemos um número real $D>d \geq 0$, por (1.1). Argumentando por contradição, suponha que a sequência $\left\{u_{n}\right\}$ não seja limitada em $H^{1}\left(\mathbb{R}^{N}\right)$, ou seja, $\left\|u_{n}\right\| \rightarrow+\infty$, quando $n \rightarrow+\infty$. 
Defina agora $\varphi_{n}:=t_{n} u_{n}$ onde $t_{n}:=\frac{2 \sqrt{D}}{\left\|u_{n}\right\|}$. Desta forma, note que

$$
\left\|\varphi_{n}\right\|=\frac{2 \sqrt{D}}{\left\|u_{n}\right\|}\left\|u_{n}\right\|=2 \sqrt{D}<\infty
$$

Então a sequência $\left\{\varphi_{n}\right\}$ é limitada em $H^{1}\left(\mathbb{R}^{N}\right)$. Para $n$ suficientemente grande, usando o Lema 1.1 (c) segue que

$$
D>I\left(u_{n}\right)=\max _{t>0} I\left(t u_{n}\right) \geq I\left(t_{n} u_{n}\right)=I\left(\varphi_{n}\right)
$$

Por outro lado,

$$
\begin{aligned}
D>I\left(\varphi_{n}\right) & =\frac{1}{2}\left\|\varphi_{n}\right\|^{2}-\int_{\mathbb{R}^{N}}(1+a(x)) F\left(\varphi_{n}\right) \mathrm{d} x \\
& =2 D-\int_{\mathbb{R}^{N}}(1+a(x)) F\left(\varphi_{n}\right) \mathrm{d} x .
\end{aligned}
$$

Assim, temos por $\left(a_{1}\right)$ que existe uma constante $C>0$ tal que

$$
D<\int_{\mathbb{R}^{N}}(1+a(x)) F\left(\varphi_{n}\right) \mathrm{d} x \leq C \int_{\mathbb{R}^{N}} F\left(\varphi_{n}\right) \mathrm{d} x
$$

Usando agora a condição $\left(f_{2}\right)$ segue a seguinte desigualdade

$$
D<C \int_{\mathbb{R}^{N}}\left(\left|\varphi_{n}\right|^{p_{1}+1}+\left|\varphi_{n}\right|^{p_{2}+1}\right) \mathrm{d} x=C\left(\left\|\varphi_{n}\right\|_{p_{1}+1}^{p_{1}+1}+\left\|\varphi_{n}\right\|_{p_{2}+1}^{p_{2}+1}\right) .
$$

Como a sequência $\left\{\varphi_{n}\right\}$ é limitada em $H^{1}\left(\mathbb{R}^{N}\right)$, temos somente dois casos a considerar:

1. $\lim _{n \rightarrow+\infty}\left(\sup _{y \in \mathbb{R}^{N}} \int_{B_{1}(y)}\left|\varphi_{n}\right|^{2} \mathrm{~d} x\right)=0$;

2. $\lim _{n \rightarrow+\infty}\left(\sup _{y \in \mathbb{R}^{N}} \int_{B_{1}(y)}\left|\varphi_{n}\right|^{2} \mathrm{~d} x\right)>0$.

Suponha que o primeiro caso seja verdade. Usando o Lema de Lions ( Lema 1.21 em [46]), temos $\varphi_{n} \rightarrow 0$ em $L^{p}\left(\mathbb{R}^{N}\right)$ para $2<p<2^{*}$. Por hipótese, temos $2<p_{1}+1 \leq p_{2}+1<2^{*}$. Isto significa que $\varphi_{n} \rightarrow 0$ em $L^{p_{i}+1}\left(\mathbb{R}^{N}\right)$, com $i=1,2$. Usando este fato, temos uma contradição com a desigualdade (1.16). Logo, a menos de subsequência, temos que

$$
\lim _{n \rightarrow+\infty}\left(\sup _{y \in \mathbb{R}^{N}} \int_{B_{1}(y)}\left|\varphi_{n}\right|^{2} \mathrm{~d} x\right)=\eta>0
$$

Fixando $n \in \mathbb{N}$, por definição de supremo, existe uma sequência $\left\{y_{n}\right\} \subset \mathbb{R}^{N}$ tal que

$$
\int_{B_{1}\left(y_{n}\right)}\left|\varphi_{n}\right|^{2} \mathrm{~d} x \geq \frac{\eta}{4}
$$

Defina a sequência $\psi_{n}:=\varphi_{n}\left(x+y_{n}\right)$ e utilizando a invariância das integrais por translação,

$$
\left\|\psi_{n}\right\|=\left(\int_{\mathbb{R}^{N}}\left|\nabla \varphi_{n}\left(x+y_{n}\right)\right|^{2}+\left|\varphi_{n}\left(x+y_{n}\right)\right|^{2}\right)^{1 / 2}=\left\|\varphi_{n}\right\|_{H^{1}\left(\mathbb{R}^{N}\right)}=2 \sqrt{D} .
$$


Em outras palavras, temos que $\left\{\psi_{n}\right\}$ é limitada em $H^{1}\left(\mathbb{R}^{N}\right)$. Pelas imersões de Sobolev e como $H^{1}\left(\mathbb{R}^{N}\right)$ é espaço reflexivo segue que

- $\psi_{n} \rightarrow \varphi$ em $H^{1}\left(\mathbb{R}^{N}\right)$;

- $\psi_{n} \rightarrow \varphi$ em $L_{l o c}^{p}\left(\mathbb{R}^{N}\right)$ para $2 \leq p<2^{*}$;

- $\psi_{n}(x) \rightarrow \varphi(x)$ q.t.p. em $\mathbb{R}^{N}$.

Consequentemente,

$$
\psi_{n} \rightarrow \varphi \quad \text { em } \quad L^{2}\left(B_{1}(0)\right)
$$

Fazendo uma mudanca de variáveis, segue por (1.17)

$$
\begin{aligned}
\int_{B_{1}(0)}|\varphi(x)|^{2} \mathrm{~d} x & =\lim _{n \rightarrow+\infty} \int_{B_{1}(0)}\left|\psi_{n}(x)\right|^{2} \mathrm{~d} x \\
& =\lim _{n \rightarrow+\infty} \int_{B_{1}(0)}\left|\varphi_{n}\left(x+y_{n}\right)\right|^{2} \mathrm{~d} x \\
& =\lim _{n \rightarrow+\infty} \int_{B_{1}\left(y_{n}\right)}\left|\varphi_{n}(x)\right|^{2} \mathrm{~d} x \geq \frac{\eta}{4}>0 .
\end{aligned}
$$

Assim existe um subconjunto $\Omega \subset \mathbb{R}^{N}$ de medida de Lebesgue positiva em $B_{1}(0)$ em que $\varphi(x) \neq$ $0, \forall x \in \Omega$. Observe que a convergência pontual em $\mathbb{R}^{N}$ nos dá

$$
0<|\varphi(x)|=\lim _{n \rightarrow+\infty}\left|\varphi_{n}\left(x+y_{n}\right)\right|=\lim _{n \rightarrow+\infty} \frac{2 \sqrt{D}\left|u_{n}\left(x+y_{n}\right)\right|}{\left\|u_{n}\left(x+y_{n}\right)\right\|}=\lim _{n \rightarrow+\infty} \frac{2 \sqrt{D}\left|u_{n}\left(x+y_{n}\right)\right|}{\left\|u_{n}\right\|}
$$

para todo $x \in \Omega$. Visto que $\left\|u_{n}\right\| \rightarrow+\infty$, quando $n \rightarrow+\infty$, necessariamente temos que

$$
\left|u_{n}\left(x+y_{n}\right)\right| \rightarrow+\infty, \text { para todo } x \in \Omega
$$

Desde que $\left\{u_{n}\right\}$ está contida em $\mathcal{N}$, podemos usar o Lema de Fatou, (1.1), a condição $\left(f_{5}\right),(1.1)$ e $\left(a_{1}\right)$ e obter

$$
\begin{aligned}
D & >\lim _{n \rightarrow+\infty} I\left(u_{n}\right)=\lim _{n \rightarrow+\infty}\left(\frac{1}{2}\left\|u_{n}\right\|^{2}-\int_{\mathbb{R}^{N}}(1+a(x)) F\left(u_{n}\right) \mathrm{d} x\right) \\
& =\lim _{n \rightarrow+\infty} \int_{\mathbb{R}^{N}}(1+a(x))\left(\frac{1}{2} f\left(u_{n}\right) u_{n}-F\left(u_{n}\right)\right) \mathrm{d} x \\
& \geq \liminf _{n \rightarrow+\infty} \int_{\mathbb{R}^{N}} \tau\left(\frac{1}{2} f\left(u_{n}\right) u_{n}-F\left(u_{n}\right)\right) \mathrm{d} x \\
& =\liminf _{n \rightarrow+\infty} \int_{\mathbb{R}^{N}} \tau\left(\frac{1}{2} f\left(u_{n}\left(x+y_{n}\right)\right) u_{n}\left(x+y_{n}\right)-F\left(u_{n}\left(x+y_{n}\right)\right)\right) \mathrm{d} x \\
& >\liminf _{n \rightarrow+\infty} \int_{\Omega} \tau\left(\frac{1}{2} f\left(u_{n}\left(x+y_{n}\right)\right) u_{n}\left(x+y_{n}\right)-F\left(u_{n}\left(x+y_{n}\right)\right)\right) \mathrm{d} x \\
& \geq \int_{\Omega} \tau \liminf _{n \rightarrow+\infty}\left(\frac{1}{2} f\left(u_{n}\left(x+y_{n}\right)\right) u_{n}\left(x+y_{n}\right)-F\left(u_{n}\left(x+y_{n}\right)\right)\right) \mathrm{d} x=+\infty .
\end{aligned}
$$

Logo, temos uma contradição e o segundo caso também não pode acontecer. A contradição veio do fato de supor que a sequência $\left\{u_{n}\right\}$ não era limitada em $H^{1}\left(\mathbb{R}^{N}\right)$ e, portanto, finalizamos a demonstração do lema. 
O resultado seguinte mostra a positividade do ínfimo do funcional associado ao problema $\left(P_{a}\right)$ sobre a variedade $\mathcal{N}$.

Lema 1.3. Seja $m$ definida em (1.6). Então tem-se $m>0$.

Demonstração: : A demonstração deste resultado requer o Lema de Lions como já utilizado no Lema 1.2. Primeiramente consideremos uma sequência minimizante $\left\{u_{n}\right\}$ na variedade de Nehari tal que $I\left(u_{n}\right) \rightarrow m$. Pelo Lema 1.2 temos que $\left\{u_{n}\right\}$ é limitada em $H^{1}\left(\mathbb{R}^{N}\right)$. Assim, utilizando as condições $\left(a_{1}\right),\left(f_{2}\right)$ existe uma constante $C>0$ tal que

$$
\begin{aligned}
0<\alpha^{2} \leq\left\|u_{n}\right\|^{2} & =\int_{\mathbb{R}^{N}}(1+a(x)) f\left(u_{n}\right) u_{n} \mathrm{~d} x \leq C \int_{\mathbb{R}^{N}}\left(\left|u_{n}\right|^{p_{1}+1}+\left|u_{n}\right|^{p_{2}+1}\right) \mathrm{d} x \\
& =C\left(\left\|u_{n}\right\|_{p_{1}+1}^{p_{1}+1}+\left\|u_{n}\right\|_{p_{2}+1}^{p_{2}+1}\right) .
\end{aligned}
$$

Argumentando como no Lema 1.2, pelo Lema de Lions, existe uma constante $\eta_{1}>0$ e uma sequência $\left\{y_{n}\right\} \in \mathbb{R}^{N}$ tais que

$$
\int_{B_{1}\left(y_{n}\right)}\left(u_{n}\right)^{2} \mathrm{~d} x=\sup _{y \in \mathbb{R}^{N}} \int_{B_{1}\left(y_{n}\right)}\left(u_{n}\right)^{2} \mathrm{~d} x \geq \frac{\eta_{1}}{4}>0 .
$$

Defina a sequência $\psi_{n}:=u_{n}\left(x+y_{n}\right)$ e note que existe uma constante $M>0$ tal que

$$
\left\|\psi_{n}\right\|=\left(\int_{\mathbb{R}^{N}}\left|\nabla u_{n}\left(x+y_{n}\right)\right|^{2}+\left|u_{n}\left(x+y_{n}\right)\right|^{2}\right)^{1 / 2}=\left\|u_{n}\right\| \leq M
$$

Em outras palavras, $\left\{\psi_{n}\right\}$ é uma sequência limitada em $H^{1}\left(\mathbb{R}^{N}\right)$. Pelas imersões de Sobolev e como $H^{1}\left(\mathbb{R}^{N}\right)$ é espaço reflexivo segue que

- $\psi_{n} \rightarrow u \mathrm{em} H^{1}\left(\mathbb{R}^{N}\right)$;

- $\psi_{n} \rightarrow u$ em $L_{l o c}^{p}\left(\mathbb{R}^{N}\right)$ para $2 \leq p<2^{*}$;

- $\psi_{n}(x) \rightarrow u(x)$ q.t.p. em $\mathbb{R}^{N}$.

Consequentemente, $\psi_{n} \rightarrow u \quad$ em $\quad L^{2}\left(B_{1}(0)\right)$.

Por mudanca de variáveis, segue que

$$
\begin{aligned}
\int_{B_{1}(0)}|u(x)|^{2} \mathrm{~d} x & =\lim _{n \rightarrow+\infty} \int_{B_{1}(0)}\left|\psi_{n}(x)\right|^{2} \mathrm{~d} x \\
& =\lim _{n \rightarrow+\infty} \int_{B_{1}(0)}\left|u_{n}\left(x+y_{n}\right)\right|^{2} \mathrm{~d} x \\
& =\lim _{n \rightarrow+\infty} \int_{B_{1}\left(y_{n}\right)}\left|u_{n}(x)\right|^{2} \mathrm{~d} x \geq \frac{\eta_{1}}{4}>0 .
\end{aligned}
$$

Assim, existe um subcontjunto $\Omega_{1}$ de medida de Lebesgue positiva em $B_{1}(0)$ no qual $u(x) \neq 0, \forall x \in$ $\Omega_{1}$. Usando o Lema de Fatou, a condição $\left(f_{3}\right)$ e $\left(a_{1}\right)$ segue que 


$$
\begin{aligned}
m & =\lim _{n \rightarrow+\infty} I\left(u_{n}\right)=\lim _{n \rightarrow+\infty}\left(\frac{1}{2}\left\|u_{n}\right\|^{2}-\int_{\mathbb{R}^{N}}(1+a(x)) F\left(u_{n}\right) \mathrm{d} x\right) \\
& =\lim _{n \rightarrow+\infty} \int_{\mathbb{R}^{N}}(1+a(x))\left(\frac{1}{2} f\left(u_{n}\right) u_{n}-F\left(u_{n}\right)\right) \mathrm{d} x \\
& \geq \liminf _{n \rightarrow+\infty} \int_{\mathbb{R}^{N}} \tau\left(\frac{1}{2} f\left(u_{n}\right) u_{n}-F\left(u_{n}\right)\right) \mathrm{d} x \\
& =\liminf _{n \rightarrow+\infty} \int_{\mathbb{R}^{N}} \tau\left(\frac{1}{2} f\left(u_{n}\left(x+y_{n}\right)\right) u_{n}\left(x+y_{n}\right)-F\left(u_{n}\left(x+y_{n}\right)\right)\right) \mathrm{d} x \\
& >\liminf _{n \rightarrow+\infty} \int_{\Omega} \tau\left(\frac{1}{2} f\left(u_{n}\left(x+y_{n}\right)\right) u_{n}\left(x+y_{n}\right)-F\left(u_{n}\left(x+y_{n}\right)\right)\right) \mathrm{d} x \\
& \geq \int_{\Omega} \tau \liminf _{n \rightarrow+\infty}\left(\frac{1}{2} f\left(u_{n}\left(x+y_{n}\right)\right) u_{n}\left(x+y_{n}\right)-F\left(u_{n}\left(x+y_{n}\right)\right)\right) \mathrm{d} x \\
& =\int_{\Omega} \tau\left(\frac{1}{2} f(u) u-F(u)\right) \mathrm{d} x>0 .
\end{aligned}
$$

Assim, segue o resultado desejado.

Lema 1.4. Suponha que u seja uma solução do problema $\left(P_{a}\right)$ com $I(u)$ no intervalo $[m, 2 m)$. Então a solução u não muda de sinal.

Demonstração: Seja $u$ uma solução fraca do problema $\left(P_{a}\right)$, então $I^{\prime}(u)=0$, isto é, $I^{\prime}(u) v=0$ para todo $v \in H^{1}\left(\mathbb{R}^{N}\right)$. Em particular, $I^{\prime}(u) u^{+}=0$ e $I^{\prime}(u) u^{-}=0$ onde consideramos $u^{+}=\max \{u, 0\} \mathrm{e}$ $u^{-}=\min \{u, 0\}$. Note que se $I^{\prime}(u) u^{+}=0$, então $\left\langle u, u^{+}\right\rangle-\int_{\mathbb{R}^{N}}(1+a(x)) f(u) u^{+} \mathrm{d} x=0$, ou seja,

$$
\left\langle u, u^{+}\right\rangle-\left\{\int_{\{u \geq 0\}}(1+a(x)) f\left(u^{+}\right) u^{+} \mathrm{d} x+\int_{\{u<0\}}(1+a(x)) f\left(u^{-}\right) u^{+} \mathrm{d} x\right\}=0,
$$

isto é, $\left\|u^{+}\right\|^{2}-\int_{\mathbb{R}^{N}}(1+a(x)) f\left(u^{+}\right) u^{+} \mathrm{d} x=0$. Portanto, $I^{\prime}\left(u^{+}\right) u^{+}=0$ e, similarmente, $I^{\prime}\left(u^{-}\right) u^{-}=0$.

Suponha que $u^{+} \neq 0$ e $u^{-} \neq 0$, então pelo que acabamos de ver temos que $u^{+}$e $u^{-}$estão na variedade $\mathcal{N}$ e ainda

$$
\begin{aligned}
I(u)= & I\left(u^{+}+u^{-}\right)=\frac{1}{2}\left\|u^{+}+u^{-}\right\|^{2}-\int_{\mathbb{R}^{N}}(1+a(x)) F\left(u^{+}+u^{-}\right) \mathrm{d} x \\
= & \frac{1}{2}\left\langle u^{+}+u^{-}, u^{+}+u^{-}\right\rangle-\int_{\{u \geq 0\}}(1+a(x)) F\left(u^{+}+u^{-}\right) \mathrm{d} x \\
& -\int_{\{u<0\}}(1+a(x)) F\left(u^{+}+u^{-}\right) \mathrm{d} x \\
= & \frac{1}{2}\left\{\left\|u^{+}\right\|^{2}+\left\|u^{-}\right\|^{2}\right\}-\int_{\{u \geq 0\}}(1+a(x)) F\left(u^{+}\right) \mathrm{d} x \\
& -\int_{\{u<0\}}(1+a(x)) F\left(u^{-}\right) \mathrm{d} x \\
= & \frac{1}{2}\left\{\left\|u^{+}\right\|^{2}+\left\|u^{-}\right\|^{2}\right\}-\int_{\mathbb{R}^{N}}(1+a(x)) F\left(u^{+}\right) \mathrm{d} x \\
& -\int_{\mathbb{R}^{N}}(1+a(x)) F\left(u^{-}\right) \mathrm{d} x \\
= & I\left(u^{+}\right)+I\left(u^{-}\right) \geq 2 m .
\end{aligned}
$$

Por outro lado, por hipótese $I(u)<2 m$ e como $m$ é estritamente positivo pelo Lema 1.3, obtemos uma contradição e assim finalizamos a demonstração . 
Observação 1.3. Note que o resultado do Lema 1.4 se aplica analogamente ao funcional $I_{\infty}$ definido em (1.8) , isto é, suponha que u é uma solução do problema $\left(P_{\infty}\right)$ com $I_{\infty}(u)$ no intervalo $\left[m_{\infty}, 2 m_{\infty}\right)$. Então a solução u não muda de sinal.

Um conceito importante e bastante conhecido que utilizaremos é o conceito de sequência de PalaisSmale. Dizemos que uma sequência $\left\{u_{n}\right\}$ em $H^{1}\left(\mathbb{R}^{N}\right)$ é uma sequência $(P S)_{c}$ para alguma constante $c$, se $I\left(u_{n}\right) \rightarrow c$ e $I^{\prime}\left(u_{n}\right) \rightarrow 0$ em $H^{-1}\left(\mathbb{R}^{N}\right)$, se $n \rightarrow+\infty$. Caso a sequência $\left\{u_{n}\right\}$ possua subsequência convergente, dizemos que o funcional $I$ satisfaz $(P S)_{c}$ no nível $c$.

O próximo lema nos dá uma importante informação sobre uma sequência $(P S)$ do funcional $I$ restrito à variedade $\mathcal{N}$ no espaco $H^{1}\left(\mathbb{R}^{N}\right)$. Aqui é o momento em que precisamos utilizar a condição de crescimento $\left(f_{2}\right)$ para a derivada de $f$.

Lema 1.5. Suponha que $\left\{u_{n}\right\}$ é uma sequência $(P S)_{d}$ para I restrito à variedade $\mathcal{N}$. Então, a menos de subsequência, a sequência $\left\{u_{n}\right\}$ é também uma sequência $(P S)_{d}$ para o funcional $I$ em $H^{1}\left(\mathbb{R}^{N}\right)$.

Demonstração: Primeiramente supomos que exista uma sequência $\left\{u_{n}\right\} \subset \mathcal{N}$ do tipo $(P S)_{d}$ para $I$ restrito à variedade $\mathcal{N}$. Por definição,

$$
I\left(u_{n}\right) \rightarrow d \text { e }(I \mid \mathcal{N})^{\prime}\left(u_{n}\right) \rightarrow 0
$$

Usando o Lema 1.2, como a sequência $\left\{u_{n}\right\} \subset \mathcal{N}$ e $I\left(u_{n}\right) \rightarrow d>0$, então a sequência $\left\{u_{n}\right\}$ é limitada em $H^{1}\left(\mathbb{R}^{N}\right)$.

Para toda $v \in H^{1}\left(\mathbb{R}^{N}\right)$, como a sequência $\left\{u_{n}\right\}$ é limitada e usando a desigualdade de Hölder e a condição de crescimento $\left(f_{2}\right)$ segue que

$$
\begin{aligned}
\left|J^{\prime}\left(u_{n}\right) v\right|= & \left|2\left\langle u_{n}, v\right\rangle-\int_{\mathbb{R}^{N}}(1+a(x))\left(f^{\prime}\left(u_{n}\right) u_{n}+f\left(u_{n}\right)\right) v \mathrm{~d} x\right| \\
\leq & 2\left\|u_{n}\right\|\|v\|+\int_{\mathbb{R}^{N}}|(1+a(x))|\left|\left(f^{\prime}\left(u_{n}\right) u_{n}+f\left(u_{n}\right)\right)\right||v| \mathrm{d} x \\
\leq & 2\left\|u_{n}\right\|\|v\|+c_{a} \int_{\mathbb{R}^{N}}\left|\left(f^{\prime}\left(u_{n}\right) u_{n}+f\left(u_{n}\right)\right)\right||v| \mathrm{d} x \\
\leq & C\left\{\|v\|\left\|u_{n}\right\|+c_{2} \int_{\mathbb{R}^{N}}\left(\left|u_{n}\right|^{p_{1}}+\left|u_{n}\right|^{p_{2}}\right)|v| \mathrm{d} x\right\} \\
= & C\left\{\|v\|\left\|u_{n}\right\|+\int_{\mathbb{R}^{N}}\left(\left|u_{n}\right|^{p_{1}}\right)|v| \mathrm{d} x+\int_{\mathbb{R}^{N}}\left(\left|u_{n}\right|^{p_{2}}\right)|v| \mathrm{d} x\right\} \\
\leq & C\left\{\|v\|\left\|u_{n}\right\|+\left(\int_{\mathbb{R}^{N}}\left(\left|u_{n}\right|^{p_{1}+1}\right) \mathrm{d} x\right)^{p_{1} /\left(p_{1}+1\right)}\left(\int_{\mathbb{R}^{N}}\left(|v|^{p_{1}+1}\right) \mathrm{d} x\right)^{1 /\left(p_{1}+1\right)}\right\} \\
& +C\left\{\left(\int_{\mathbb{R}^{N}}\left(\left|u_{n}\right|^{p_{2}+1}\right) \mathrm{d} x\right)^{p_{2} /\left(p_{2}+1\right)}\left(\int_{\mathbb{R}^{N}}\left(|v|^{p_{2}+1}\right) \mathrm{d} x\right)^{1 /\left(p_{2}+1\right)}\right\} \\
= & C\left\{\|v\|\left\|u_{n}\right\|+\left\|u_{n}\right\|_{p_{1}+1}^{p_{1}}\|v\|_{p_{1}+1}+\left\|u_{n}\right\|_{p_{2}+1}^{p_{2}}\|v\|_{p_{2}+1}\right\} \\
\leq & C\left\{\|v\|\left\|u_{n}\right\|+\left\|u_{n}\right\|^{p_{1}}\|v\|+\left\|u_{n}\right\|^{p_{2}}\|v\|\right\} .
\end{aligned}
$$

Como a sequência $\left\{u_{n}\right\}$ é limitada, então para toda $v \in H^{1}\left(\mathbb{R}^{N}\right)$,

$$
\left|J^{\prime}\left(u_{n}\right) v\right| \leq C\|v\|
$$


Isto mostra que a sequência $\left\{J^{\prime}\left(u_{n}\right)\right\}$ é limitada em $H^{-1}\left(\mathbb{R}^{N}\right)$. Consequentemente,

$$
\left|J^{\prime}\left(u_{n}\right) u_{n}\right| \leq\left\|J^{\prime}\left(u_{n}\right)\right\|_{H^{-1}\left(\mathbb{R}^{N}\right)}\left\|u_{n}\right\|_{H^{1}\left(\mathbb{R}^{N}\right)} \leq C,
$$

em que a constante $C$ não depende de $\left\{u_{n}\right\}$. A menos de subsequência, a sequência de números reais positivos

$$
\lambda_{n}:=\left|J^{\prime}\left(u_{n}\right) u_{n}\right| \rightarrow \lambda \geq 0
$$

Além disso, como $\left\{u_{n}\right\} \subset \mathcal{N}$, pelo Lema 1.1(a) temos que $\left\|u_{n}\right\| \geq \alpha>0$ e argumentando como na demonstração do Lema 1.3, usando o Lema de Lions, $\left(a_{1}\right)$ e $\left(f_{3}\right)$ segue que $u_{n}(x) \rightarrow u(x)$ q.t.p. $x \in \mathbb{R}^{N}$ e $u(x) \neq 0$ q.t.p. $x \in \Omega_{0}$ onde $\Omega_{0}$ é um conjunto de medida positiva . Daí usando o Lema de Fatou e o fato de que $f^{\prime}(t) t-f(t) \geq 0$ para todo $t \in \mathbb{R}$,

$$
\begin{aligned}
\lambda & =\lim _{n \rightarrow+\infty}\left|J^{\prime}\left(u_{n}\right) u_{n}\right| \\
& =\lim _{n \rightarrow+\infty}\left|\left\{2\left\|u_{n}\right\|^{2}-\int_{\mathbb{R}^{N}}(1+a(x))\left(f^{\prime}\left(u_{n}\right) u_{n}+f\left(u_{n}\right)\right) u_{n} \mathrm{~d} x\right\}\right| \\
& =\lim _{n \rightarrow+\infty}\left|\int_{\mathbb{R}^{N}}(1+a(x))\left(f^{\prime}\left(u_{n}\right) u_{n}^{2}-f\left(u_{n}\right) u_{n}\right) \mathrm{d} x\right| \\
& \geq \tau \lim _{n \rightarrow+\infty} \int_{\Omega_{0}}\left(f^{\prime}\left(u_{n}(x)\right) u_{n}^{2}(x)-f\left(u_{n}(x)\right) u_{n}(x)\right) \mathrm{d} x \\
& =\tau \int_{\Omega_{0}}\left(f^{\prime}(u) u^{2}-f(u) u\right) \mathrm{d} x \\
& =\tau \int_{\Omega_{0}}\left(f^{\prime}(u) u-f(u)\right) u \mathrm{~d} x>0
\end{aligned}
$$

Como $\mathcal{N}$ é uma variedade de codimensão 1 em $H^{1}\left(\mathbb{R}^{N}\right)$ (vide seção 6.3 em [7]), podemos escrever a projeção gradiente $\left(\left.I\right|_{\mathcal{N}}\right)^{\prime}(u)$ sobre o plano tangente

$$
T_{u} \mathcal{N}=\left\{v \in H^{1}\left(\mathbb{R}^{N}\right):\left\langle J^{\prime}(u), v\right\rangle=0\right\}
$$

por

$$
\left(\left.I\right|_{\mathcal{N}}\right)^{\prime}(u)=I^{\prime}(u)-t J^{\prime}(u)
$$

em que

$$
t:=\frac{\left\langle I^{\prime}(u), J^{\prime}(u)\right\rangle}{\left\|J^{\prime}(u)\right\|^{2}} .
$$

Desde que $\left\{u_{n}\right\} \subset \mathcal{N}$ é uma sequência $(P S)$ de $I$ restrito à variedade de Nehari (pelo Lema 7.19 em [7]) existe uma sequência $\left\{t_{n}\right\}$ tal que

$$
\left(\left.I\right|_{\mathcal{N}}\right)^{\prime}\left(u_{n}\right)=I^{\prime}\left(u_{n}\right)-t_{n} J^{\prime}\left(u_{n}\right)
$$

dada por

$$
t_{n}:=\frac{\left\langle I^{\prime}\left(u_{n}\right), J^{\prime}\left(u_{n}\right)\right\rangle}{\left\|J^{\prime}\left(u_{n}\right)\right\|^{2}}
$$

note que $\left\|J^{\prime}\left(u_{n}\right)\right\| \neq 0$ por $(1.15)$.

O objetivo agora é mostrar que a sequência $\left\{t_{n}\right\}$ converge a zero se $n \rightarrow+\infty$ e assim concluiremos o 
lema. Por (1.18) e (1.21) segue que

$$
0=I^{\prime}\left(u_{n}\right) u_{n}=\left(\left.I\right|_{\mathcal{N}}\right)^{\prime}\left(u_{n}\right) u_{n}+t_{n} J^{\prime}\left(u_{n}\right) u_{n}=o_{n}(1)+t_{n} J^{\prime}\left(u_{n}\right) u_{n}
$$

Por (1.19) e (1.20) tem-se

$$
C \geq\left|J^{\prime}\left(u_{n}\right) u_{n}\right| \rightarrow \lambda>0, \text { se } n \rightarrow+\infty,
$$

e por (1.22) isto implica que, a menos de subsequência,

$$
t_{n} \rightarrow 0, \text { se } n \rightarrow+\infty
$$

Assim, deduzimos de (1.18) e (1.23) que, a menos de subsequência de $\left\{u_{n}\right\}$,

$$
I^{\prime}\left(u_{n}\right)=\left(\left.I\right|_{\mathcal{N}}\right)^{\prime}\left(u_{n}\right)+t_{n} J^{\prime}\left(u_{n}\right) \rightarrow 0, \text { se } n \rightarrow+\infty .
$$

Concluímos assim a demonstração do lema.

Os resultados clássicos relativos ao problema limite $\left(P_{\infty}\right)$ encontrados em [12], [13] , e [25] juntamente com o estudo da simetria radial e decaimento exponencial podem ser resumidos da seguinte forma: o problema tem uma solução de energia mínima $\omega$ tal que

i) $\omega>0 \mathrm{em} \mathbb{R}^{N}$;

ii) $\omega$ é radialmente simétrica: $\omega(x)=\omega(r)$, onde $r=|x|$ e $\omega$ decresce com respeito a $r$;

iii) $\omega \in C^{2}\left(\mathbb{R}^{N}\right)$;

iv) existem constantes $C_{1}>0$ e $C_{2}>0$ satisfazendo

$$
C_{1}(1+|x|)^{-\frac{N-1}{2}} e^{-|x|} \leq \omega(x) \leq C_{2}(1+|x|)^{-\frac{N-1}{2}} e^{-|x|}, \quad \forall x \in \mathbb{R}^{N} .
$$

Além disso, $\omega$ é único dependendo das hipóteses sobre $f$ como em [26, 40].

Lema 1.6. Não existe solução u para o problema $\left(P_{\infty}\right)$ tal que $I_{\infty}(u) \in\left(m_{\infty}, 2 m_{\infty}\right)$.

Demonstração: Se $u$ é solução do problema $\left(P_{\infty}\right)$ tal que $I_{\infty}(u) \in\left(m_{\infty}, 2 m_{\infty}\right)$, então pela Observação 1.3, $u$ não muda de sinal, isto é, $u>0$ ou $u<0$. Sem perda de generalidade, considere $u>0$ ( no caso $u<0$, como $f$ é ímpar, segue que $-u>0$ é solução). Pelos resultados clássicos, $u$ é solução positiva, radialmente simétrica de $\left(P_{\infty}\right)$ e única, portanto, $u=\omega$ e $I_{\infty}(u)=m_{\infty}$ implicando em contradição com a hipótese.

\subsection{Compacidade}

O próximo resultado que enunciaremos será fundamental para o estudo dos pontos críticos do funcional $I$. Ele descreve como uma sequência de Palais - Smale de $I$ se comporta assintoticamente. 
Lema 1.7. (Lema de Splitting) Suponha que $\left\{u_{n}\right\}$ é uma sequência limitada em $H^{1}\left(\mathbb{R}^{N}\right)$ tal que

$$
I\left(u_{n}\right) \rightarrow d>0 \quad e \quad\left(\left.I\right|_{\mathcal{N}}\right)^{\prime}\left(u_{n}\right) \rightarrow 0 \quad \text { em } \quad H^{-1}\left(\mathbb{R}^{N}\right)
$$

Então, passando se necessário a uma subsequência, existem $u_{0}$ solução fraca de $\left(P_{a}\right)$, um número $k \in$ $\mathbb{N} \cup\{0\}, k$ funções $w^{1}, \ldots, w^{k} \in H^{1}\left(\mathbb{R}^{N}\right)$ soluções do problema limite $\left(P_{\infty}\right)$ e $k$ sequências de pontos $\left\{y_{n}^{j}\right\}, 1 \leq j \leq k$ tais que

1. $u_{n} \rightarrow u_{0}$ em $H^{1}\left(\mathbb{R}^{N}\right)$ ou

2. $\left|y_{n}^{j}\right| \rightarrow+\infty e\left|y_{n}^{j}-y_{n}^{i}\right| \rightarrow+\infty ; j \neq i$

3. $u_{n}-\sum_{j=1}^{k} w^{j}\left(\cdot-y_{n}^{j}\right) \rightarrow u_{0} \quad$ em $H^{1}\left(\mathbb{R}^{N}\right)$;

4. $I\left(u_{n}\right) \rightarrow d=I\left(u_{0}\right)+\sum_{j=1}^{k} I_{\infty}\left(w^{j}\right)$.

Demonstração: O primeiro passo da demonstração é o Lema 1.5. A demonstração segue como em [33].

Como uma consequência dos Lemas 1.2 , 1.5 e 1.6 combinados com o Lema 1.7 temos o seguinte resultado.

Lema 1.8. Suponha que o infimo $m$ definido em (1.6) não seja atingido. Então $m \geq m_{\infty} e$, ainda, o funcional I satisfaz a condição de Palais-Smale sobre $\mathcal{N}$ em qualquer nível do intervalo $\left(m_{\infty}, 2 m_{\infty}\right)$.

Demonstração: Considere uma sequência $\left\{u_{n}\right\}$ tal que satisfaz $(P S)_{d}$ para o funcional $I$ restrito a $\mathcal{N}$. Então, a menos de subsequência, $\left\{u_{n}\right\}$ é limitada e é $(P S)_{d}$ para $I$ em todo o espaço $H^{1}\left(\mathbb{R}^{N}\right)$ pelos Lemas 1.2 e 1.5 .

Suponha que o nível $m$ não seja atingido. Se existisse uma sequência $u_{n} \rightarrow u_{0}$, pela continuidade do funcional $I$, teríamos $I\left(u_{n}\right) \rightarrow I\left(u_{0}\right)=d=m$. Contradição com a hipótese neste caso. Logo, não existe tal sequência; o que implica que a possibilidade (1) no Lema de Splitting não ocorre. Decorre então que valem $(2),(3)$ e (4).

Em primeiro lugar, suponha que $u_{0}=0$, então por (4), temos que

$$
I\left(u_{n}\right) \rightarrow d=m=I\left(u_{0}\right)+\sum_{j=1}^{k} I_{\infty}\left(w^{j}\right) \geq 0+k I_{\infty}(w) \geq k m_{\infty} \geq m_{\infty}
$$

onde $w$ é a solução de energia mínima para $I_{\infty}$.

Em segundo lugar, assuma que $u_{0} \neq 0$. Como $u_{0}$ pertence a $\mathcal{N}$, tem-se $I\left(u_{0}\right)>0$. Segue que $m>k m_{\infty} \geq m_{\infty}$. Logo, em ambos os casos, temos $m \geq m_{\infty}$ e assim a primeira parte do Lema está verificada.

Suponha que $d \in\left(m_{\infty}, 2 m_{\infty}\right)$. Assim, para $n$ suficientemente grande,

$$
m_{\infty}<I\left(u_{n}\right)<2 m_{\infty}
$$


Se não existisse subsequência $\left\{u_{n}\right\}$ convergente, usando o Lema 1.7,

$$
I\left(u_{n}\right) \rightarrow d=I\left(u_{0}\right)+\sum_{j=1}^{k} I_{\infty}\left(w^{j}\right) .
$$

Se $k \geq 2$, então

$$
I\left(u_{n}\right) \rightarrow d=I\left(u_{0}\right)+\sum_{j=1}^{k} I_{\infty}\left(w^{j}\right) \geq 0+k I_{\infty}(w) \geq k m_{\infty} \geq 2 m_{\infty} .
$$

Isto configura um absurdo uma vez que $d<2 m_{\infty}$. Portanto, obrigatoriamente, tem-se $k=1$. Neste caso, se $u_{0}=0$, então

$$
2 m_{\infty}>I_{\infty}\left(w^{j}\right)=d>m_{\infty} .
$$

Isto é uma contradição com o Lema 1.6, pois não existe solução $w^{j}$ de $\left(P_{\infty}\right)$ no intervalo $\left(m_{\infty}, 2 m_{\infty}\right)$. Usando mais uma vez o Lema 1.7 , como $u_{0}$ pertence a $\mathcal{N}$,

$$
I\left(u_{n}\right) \rightarrow d=I\left(u_{0}\right)+1 . I_{\infty}\left(w^{j}\right) \geq m+I_{\infty}(w)=m+m_{\infty} \geq m_{\infty}+m_{\infty}=2 m_{\infty} .
$$

Isto também configura uma contradição, visto que $d<2 m_{\infty}$. Neste caso, concluimos que $k$ não pode ser maior ou igual a 1 e devemos ter $k=0$ e, portanto, por (3) existe uma subsequência de $\left\{u_{n}\right\}$ convergente e o lema está provado.

\subsection{Projeção sobre Nehari}

O próximo passo é mostrar que o conjunto $\mathcal{N}$ é não vazio. Com tal objetivo, iremos trabalhar com $y_{0} \in \mathbb{R}^{N}$ fixado, $\left\|y_{0}\right\|=1, y \in \partial B_{2}\left(y_{0}\right)$ onde $B_{2}\left(y_{0}\right):=\left\{x \in \mathbb{R}^{N} ;\left\|x-y_{0}\right\| \leq 2\right\}$ e com $0 \leq \lambda \leq 1$.

Como o problema limite $\left(P_{\infty}\right)$ tem uma solução ground state $w>0$, trabalhamos com esta solução transladada, isto é,

$$
w_{0}^{R}:=w\left(x-R y_{0}\right)
$$

e

$$
w_{y}^{R}:=w(x-R y)
$$

e definimos uma combinação linear

$$
z_{\lambda, y}^{R}:=\lambda w\left(x-R y_{0}\right)+(1-\lambda) w(x-R y)=\lambda w_{0}^{R}+(1-\lambda) w_{y}^{R} .
$$

Para demonstrarmos a projeção sobre $\mathcal{N}$ precisamos utilizar dois resultados. O primeiro deles é

Proposição 1.1. Considere dois números reais $r \in(0,+\infty)$ e $\lambda \in[0,1]$ e defina a seguinte função em duas variáveis

$$
\Phi(\lambda, r):=\lambda^{2}\left(\|w\|^{2}-\int_{\mathbb{R}^{N}} \frac{f(r \lambda w)}{r \lambda w} w^{2} \mathrm{~d} x\right),
$$


onde $w$ é a solução ground state do problema $\left(P_{\infty}\right)$. Então existe um número $S_{0}<0$ e $T_{0}>0$ de modo que $\forall r \geq T_{0}$ tem-se

$$
\Phi(\lambda, r)+\Phi(1-\lambda, r) \leq S_{0}<0
$$

Demonstração: Começamos observando que decorre da hipótese $\left(f_{3}\right)$ que a função $\Phi$ é decrescente na variável $r$. Por outro lado, derivando a função $\Phi$ em (1.26) com respeito a variável $\lambda$, fixando $r$, obtem-se

$$
\begin{aligned}
\frac{\partial \Phi}{\partial \lambda}(\lambda, r)= & 2 \lambda\left(\|w\|^{2}-\int_{\mathbb{R}^{N}} \frac{f(r \lambda w)}{r \lambda w} w^{2} \mathrm{~d} x\right) \\
& +\lambda^{2}\left(-\int_{\mathbb{R}^{N}} \frac{f^{\prime}(r \lambda w) r w r \lambda w-f(r \lambda w) r w}{(r \lambda w)^{2}} w^{2}\right)
\end{aligned}
$$

Como $w$ é a solução positiva ground state do problema $\left(P_{\infty}\right)$, então $w$ satisfaz

$$
-\Delta w+w=f(w)
$$

Segue que

$$
\|w\|^{2}=\int_{\mathbb{R}^{N}}\left[|\nabla w|^{2}+w^{2}\right] \mathrm{d} x=\int_{\mathbb{R}^{N}} f(w) w \mathrm{~d} x=\int_{\mathbb{R}^{N}} \frac{f(w)}{w} w^{2} \mathrm{~d} x .
$$

Substituindo (1.28) em (1.27), tem-se

$$
\begin{aligned}
\frac{\partial \Phi}{\partial \lambda}(\lambda, r)= & 2 \lambda \int_{\mathbb{R}^{N}}\left(\frac{f(w)}{w}-\frac{f(r \lambda w)}{r \lambda w}\right) w^{2} \mathrm{~d} x \\
& +\lambda^{2}\left(-\int_{\mathbb{R}^{N}}\left[\frac{f^{\prime}(r \lambda w)}{\lambda}-\frac{f(r \lambda w)}{r \lambda^{2} w}\right] w^{2} \mathrm{~d} x\right) \\
= & 2 \lambda \int_{\mathbb{R}^{N}}\left(\frac{f(w)}{w}-\frac{f(r \lambda w)}{r \lambda w}\right) w^{2} \mathrm{~d} x \\
& +\int_{\mathbb{R}^{N}}\left[-f^{\prime}(r \lambda w) \lambda+\frac{f(r \lambda w)}{r w}\right] w^{2} \mathrm{~d} x \\
= & \int_{\mathbb{R}^{N}}\left[2 \lambda \frac{f(w)}{w}-f^{\prime}(r \lambda w) \lambda-\frac{\lambda}{\lambda} \frac{f(r \lambda w)}{r w}\right] w^{2} \mathrm{~d} x \\
= & \int_{\mathbb{R}^{N}}\left[2 \lambda \frac{f(w)}{w}-\left(f^{\prime}(r \lambda w) \lambda+\lambda \frac{f(r \lambda w)}{r \lambda w}\right)\right] w^{2} \mathrm{~d} x .
\end{aligned}
$$

Por $\left(f_{3}\right)$, se $u>0$ temos $f^{\prime}(u)>\frac{f(u)}{u}$, e assim

$$
f^{\prime}(u)+\frac{f(u)}{u}>2 \frac{f(u)}{u} .
$$

Isto implica que $\forall \lambda \in(0,1]$,

$$
-\lambda\left(f^{\prime}(u)+\frac{f(u)}{u}\right)<-2 \lambda \frac{f(u)}{u}
$$

Agora escolhendo $u=r \lambda w>0$ em (1.30) e substituindo na derivada da $\Phi$, isto é, em (1.29) tem-se que 


$$
\begin{aligned}
\frac{\partial \Phi}{\partial \lambda}(\lambda, r) & =\int_{\mathbb{R}^{N}}\left[2 \lambda \frac{f(w)}{w}-\left(f^{\prime}(r \lambda w) \lambda+\frac{\lambda}{\lambda} \frac{f(r \lambda w)}{r w}\right)\right] w^{2} \mathrm{~d} x \\
& <\int_{\mathbb{R}^{N}}\left[2 \lambda \frac{f(w)}{w}-2 \lambda \frac{f(r \lambda w)}{r \lambda w}\right] w^{2} \mathrm{~d} x \\
& =2 \lambda \int_{\mathbb{R}^{N}}\left[\frac{f(w)}{w}-\frac{f(r \lambda w)}{r \lambda w}\right] w^{2} \mathrm{~d} x .
\end{aligned}
$$

Escolhendo $r$ suficientemente grande tal que $r \lambda>1$ e usando, mais uma vez, a condição $\left(f_{3}\right)$ que implica $\frac{f(t)}{t}$ crescente para $t>0$ segue que

$$
\frac{\partial \Phi}{\partial \lambda}(\lambda, r)<0
$$

Portanto, $\Phi$ é decrescente na variável $\lambda$.

Decorre da definição de $\Phi$ e pela condição $\left(f_{3}\right)$ que existe uma constante $M_{1}>0$ tal que para todo $\lambda \in(0,1]$ e $r \in(0,+\infty)$ temos

$$
\Phi(\lambda, r) \leq \lambda^{2}\|w\|^{2}:=M_{1} \lambda^{2} .
$$

Por outro lado, como $w$ é solução positiva do problema $\left(P_{\infty}\right)$, para todo $0<\lambda \leq 1$, tem-se por $\left(f_{3}\right)$ e $\left(f_{4}\right)$ que

$$
\lim _{r \rightarrow+\infty} \frac{f(r \lambda w)}{r \lambda w} \geq l_{\infty}
$$

e $\frac{f(r \lambda w)}{r \lambda w}$ é crescente na variável $r$. Isto implica que no caso assintoticamente linear $\frac{f(r \lambda w)}{r \lambda w} \nearrow l_{\infty} \mathrm{e}$

$$
\begin{aligned}
\left|\frac{f(w)}{w}-\frac{f(r \lambda w)}{r \lambda w}\right| w^{2} & \leq\left(\left|\frac{f(w)}{w}\right|+\left|\frac{f(r \lambda w)}{r \lambda w}\right|\right) w^{2} \\
& \leq 2 l_{\infty} w^{2} \in L^{1}\left(\mathbb{R}^{N}\right) .
\end{aligned}
$$

Usando o Teorema da Convergência Dominada de Lebesgue, existe uma constante $M_{2}>0$, tal que

$$
\begin{aligned}
\lim _{r \rightarrow+\infty} \Phi(\lambda, r) & =\lim _{r \rightarrow+\infty} \lambda^{2} \int_{\mathbb{R}^{N}}\left[\frac{f(w)}{w}-\frac{f(r \lambda w)}{r \lambda w}\right] w^{2} \mathrm{~d} x \\
& =\lambda^{2} \int_{\mathbb{R}^{N}}\left[\frac{f(w)}{w}-l_{\infty}\right] w^{2} \mathrm{~d} x:=\lambda^{2}\left(-M_{2}\right) .
\end{aligned}
$$

Para o caso superlinear, visto que $\frac{f(s)}{s}$ é crescente se $s>0$, usamos o Teorema da Convergência Monótona para obter o resultado em (1.33).

Vamos considerar $\lambda$ no intervalo $\left[0, \frac{1}{2}\right]$ devido à simetria de $\Phi(\lambda, r)+\Phi(1-\lambda, r)$ com respeito à $\lambda$. Começamos fixando $\lambda_{0}$ no intervalo $\left(0, \frac{1}{2}\right)$ tal que

$$
M_{1} \lambda_{0}^{2}<\frac{M_{2}}{2}\left(1-\lambda_{0}\right)^{2} ;
$$

pode-se encontrar $r_{0}>2$ satisfazendo 


$$
\Phi\left(1-\lambda_{0}, r_{0}\right)=-\frac{M_{2}}{2}\left(1-\lambda_{0}\right)^{2}
$$

De fato, se $0<\lambda_{0}<\frac{1}{2}$, então $\left(1-\lambda_{0}\right)^{2}>\frac{1}{4}$. Como $M_{2}>0$, escolha $\lambda_{0}<\sqrt{\frac{M_{2}}{8 M_{1}}}$. Assim, $\left(1-\lambda_{0}\right)^{2} \frac{M_{2}}{2}>$ $\frac{M_{2}}{8}$ e segue que

$$
M_{1} \lambda_{0}^{2}<M_{1} \frac{M_{2}}{8 M_{1}}=\frac{M_{2}}{8}<\left(1-\lambda_{0}\right)^{2} \frac{M_{2}}{2} .
$$

Considere agora $\lambda \in\left(0, \lambda_{0}\right]$ e $r \geq r_{0}$. Como $\frac{1}{2}<1-\lambda_{0} \leq 1-\lambda$, $\Phi$ é decrescente em $r$ e em $\lambda$, e por (1.31) segue que

$$
\begin{aligned}
\Phi(\lambda, r)+\Phi(1-\lambda, r) & \leq \Phi\left(\lambda, r_{0}\right)+\Phi\left(1-\lambda, r_{0}\right) \\
& \leq M_{1} \lambda_{0}^{2}+\Phi\left(1-\lambda, r_{0}\right) \\
& =M_{1} \lambda_{0}^{2}-\frac{M_{2}}{2}\left(1-\lambda_{0}\right)^{2}<0 .
\end{aligned}
$$

Por outro lado, se $\lambda \in\left[\lambda_{0}, \frac{1}{2}\right]$ e fixando $r_{1}>\max \left\{\frac{1}{\lambda_{0}}, 2\right\}$, note que se

$$
r \geq r_{1}>\frac{1}{\lambda_{0}} \Longrightarrow r \lambda \geq r_{1} \lambda \geq r_{1} \lambda_{0}>1
$$

e como

$$
r \geq r_{1}>2 \text { e } 1-\lambda \geq \frac{1}{2}
$$

então

$$
r(1-\lambda) \geq \frac{r}{2}>\frac{2}{2}=1
$$

Visto que $\frac{f(t)}{t}$ é crescente, pela definição de $\Phi,(1.36)$ e (1.37) segue que $\Phi$ é negativa, isto é, $\Phi(\lambda, r)<0$ e $\Phi(1-\lambda, r)<0$. Assim, se $\lambda \in\left[\lambda_{0}, \frac{1}{2}\right]$ segue que

$$
\Phi(\lambda, r)+\Phi(1-\lambda, r)<0
$$

Para concluir, defina $T_{0}:=\max \left\{r_{0}, r_{1}\right\}$. Logo, $T_{0}>2$ e observe que

$$
S_{0}:=\max \left\{\Phi\left(\lambda, T_{0}\right)+\Phi\left(1-\lambda, T_{0}\right)\right\}<0
$$

Assim concluimos que para $r \geq T_{0}$

$$
\Phi(\lambda, r)+\Phi(1-\lambda, r) \leq \Phi\left(\lambda, T_{0}\right)+\Phi\left(1-\lambda, T_{0}\right) \leq S_{0}<0, \text { para todo } \lambda \in[0,1]
$$

Portanto, a proposição fica provada.

O resultado que segue pode ser encontrado em [1], Lema 2.1.

Lema 1.9. Suponha que existam $\mu_{2}>\mu_{1} \geq 0$. Então, para todo $x_{1}, x_{2} \in \mathbb{R}^{N}$, existe um número real $C>0$ tal que 


$$
\int_{\mathbb{R}^{N}} e^{-\mu_{1}\left|x-x_{1}\right|} e^{-\mu_{2}\left|x-x_{2}\right|} \mathrm{d} x \leq C e^{-\mu_{1}\left|x_{1}-x_{2}\right|}
$$

Outrossim, se $\mu_{2} \geq \mu_{1}>0$ e $\mu_{3}>\mu_{1} \geq 0$, então, para quaisquer $x_{1}, x_{2}, x_{3} \in \mathbb{R}^{N}$, existe um número real $C>0$ tal que

$$
\int_{\mathbb{R}^{N}} e^{-\mu_{1}\left|x-x_{1}\right|} e^{-\mu_{2}\left|x-x_{2}\right|} e^{-\mu_{3}\left|x-x_{3}\right|} \mathrm{d} x \leq C e^{-\frac{\mu_{1}}{2}\left\{\left|x_{1}-x_{2}\right|+\left|x_{2}-x_{3}\right|+\left|x_{3}-x_{1}\right|\right\}} .
$$

O próximo resultado garante que a variedade $\mathcal{N}$ é não vazia.

Lema 1.10. Existem números $R_{0}>0, T_{0}>2$ e para cada $R \geq R_{0}, y \in \partial B_{2}\left(y_{0}\right), 0 \leq \lambda \leq 1$, um único $T_{\lambda, y}^{R}>0$ satisfazendo

$$
T_{\lambda, y}^{R} z_{\lambda, y}^{R} \in \mathcal{N}
$$

Ademais, $T_{\lambda, y}^{R} \in\left[0, T_{0}\right)$ e $T_{\lambda, y}^{R}$ é uma função contínua nas variáveis $\lambda, y, R$.

Demonstração: Sejam $u, v \in H^{1}\left(\mathbb{R}^{N}\right) ; u, v>0$ e $r \in(0,+\infty)$ em (1.10), então

$$
\begin{aligned}
J_{\infty}(r u+r v) & =\|r u+r v\|^{2}-\int_{\mathbb{R}^{N}} f(r u+r v)(r u+r v) \mathrm{d} x \\
& =\|r u+r v\|^{2}-\int_{\mathbb{R}^{N}} f(r u+r v)(r u+r v)\left(\frac{r u+r v}{r u+r v}\right) \mathrm{d} x \\
& =r^{2}\left(\|u\|^{2}+\|v\|^{2}+2\langle u, v\rangle\right)-\int_{\mathbb{R}^{N}} \frac{f(r u+r v)}{r u+r v}(r u+r v)^{2} \mathrm{~d} x \\
& =r^{2}\left(\|u\|^{2}+\|v\|^{2}+2\langle u, v\rangle\right)-\int_{\mathbb{R}^{N}} \frac{f(r u+r v)}{r u+r v}\left(r^{2} u^{2}+2 r^{2} u v+r^{2} v^{2}\right) \mathrm{d} x \\
& =r^{2}\left(\|u\|^{2}+\|v\|^{2}+2\langle u, v\rangle\right)-r^{2} \int_{\mathbb{R}^{N}} \frac{f(r u+r v)}{r u+r v}\left(u^{2}+2 u v+v^{2}\right) \mathrm{d} x .
\end{aligned}
$$

Por $\left(f_{3}\right), \frac{f(s)}{s}$ é crescente se $s>0$, assim

$$
\begin{aligned}
\frac{J_{\infty}(r u+r v)}{r^{2}}= & \|u\|^{2}+\|v\|^{2}+2\langle u, v\rangle-\int_{\mathbb{R}^{N}} \frac{f(r u+r v)}{r u+r v}\left(u^{2}+2 u v+v^{2}\right) \mathrm{d} x \\
\leq & \|u\|^{2}+\|v\|^{2}+2\langle u, v\rangle \\
& \quad-\int_{\mathbb{R}^{N}} \frac{f(r u)}{r u}\left(u^{2}\right) \mathrm{d} x-2 \int_{\mathbb{R}^{N}} \frac{f(r u+r v)}{r u+r v}(u v) \mathrm{d} x-\int_{\mathbb{R}^{N}} \frac{f(r v)}{r v}\left(v^{2}\right) \mathrm{d} x \\
& <\|u\|^{2}-\int_{\mathbb{R}^{N}} \frac{f(r u)}{r u}\left(u^{2}\right) \mathrm{d} x+\|v\|^{2}-\int_{\mathbb{R}^{N}} \frac{f(r v)}{r v}\left(v^{2}\right) \mathrm{d} x+2\langle u, v\rangle .
\end{aligned}
$$

Como o resultado em (1.38) é válido para todo $u, v \in H^{1}\left(\mathbb{R}^{N}\right) ; u, v>0$ e $r \in(0,+\infty)$, podemos substituir $u$ por $\lambda w_{0}^{R}$ e $v$ por $(1-\lambda) w_{y}^{R}$ para $0 \leq \lambda \leq 1$ e utilizando mudança de variáveis obter a seguinte desigualdade 


$$
\begin{aligned}
\frac{J_{\infty}(r u+r v)}{r^{2}}= & \frac{J_{\infty}\left(r \lambda w_{0}^{R}+r(1-\lambda) w_{y}^{R}\right)}{r^{2}} \\
\leq & \left\|\lambda w_{0}^{R}\right\|^{2}-\int_{\mathbb{R}^{N}} \frac{f\left(r \lambda w_{0}^{R}\right)}{r \lambda w_{0}^{R}}\left(\lambda w_{0}\right)^{2} \mathrm{~d} x \\
& +\left\|(1-\lambda) w_{y}^{R}\right\|^{2}-\int_{\mathbb{R}^{N}} \frac{f\left(r(1-\lambda) w_{y}^{R}\right)}{r(1-\lambda) w_{y}^{R}}\left((1-\lambda) w_{y}^{R}\right)^{2} \mathrm{~d} x+2\left\langle\lambda w_{0}^{R},(1-\lambda) w_{y}^{R}\right\rangle \\
= & \lambda^{2}\left(\left\|w_{0}^{R}\right\|^{2}-\int_{\mathbb{R}^{N}} \frac{f\left(r \lambda w_{0}^{R}\right)}{r \lambda w_{0}^{R}}\left(w_{0}^{R}\right)^{2} \mathrm{~d} x\right) \\
& +(1-\lambda)^{2}\left(\left\|w_{y}^{R}\right\|^{2}-\int_{\mathbb{R}^{N}} \frac{f\left(r(1-\lambda) w_{y}^{R}\right)}{r(1-\lambda) w_{y}^{R}}\left(w_{y}^{R}\right)^{2} \mathrm{~d} x\right)+2 \lambda(1-\lambda)\left\langle w_{0}^{R}, w_{y}^{R}\right\rangle \\
= & \lambda^{2}\left(\|w\|^{2}-\int_{\mathbb{R}^{N}} \frac{f(r \lambda w)}{r \lambda w}(w)^{2} \mathrm{~d} x\right) \\
& +(1-\lambda)^{2}\left(\|w\|^{2}-\int_{\mathbb{R}^{N}} \frac{f(r(1-\lambda) w)}{r(1-\lambda) w}(w)^{2} \mathrm{~d} x\right)+2 \lambda(1-\lambda)\left\langle w_{0}^{R}, w_{y}^{R}\right\rangle .
\end{aligned}
$$

Visto que $w$ solução do problema limite, então

$$
w_{y-y_{0}}^{R}=w\left(x-R\left(y-y_{0}\right)\right) \rightarrow 0, \text { se } R \rightarrow+\infty .
$$

$$
w_{y-y_{0}}^{R}=w\left(x-R\left(y-y_{0}\right)\right) \rightarrow 0, \text { em } H^{-1}\left(\mathbb{R}^{N}\right) .
$$

Assim, por definição de convergência fraca, para toda $\varphi \in H^{1}\left(\mathbb{R}^{N}\right)$

$$
\left\langle w\left(\cdot-R\left(y-y_{0}\right)\right), \varphi\right\rangle \rightarrow\langle 0, \varphi\rangle=0, \text { se } R \rightarrow+\infty .
$$

Em particular, escolha $\varphi=w \in H^{1}\left(\mathbb{R}^{N}\right)$ e segue que

$$
\left\langle w\left(\cdot-R\left(y-y_{0}\right)\right), w\right\rangle \rightarrow 0, \text { se } R \rightarrow+\infty .
$$

Fazendo uma mudança de variáveis, segue que

$$
\begin{aligned}
o_{R}(1) & =\left\langle w\left(\cdot-R\left(y-y_{0}\right)\right), w\right\rangle \\
& =\int_{\mathbb{R}^{N}}\left[\nabla w\left(x-R\left(y-y_{0}\right)\right) \nabla w(x)+w\left(x-R\left(y-y_{0}\right)\right) w(x)\right] \mathrm{d} x \\
& =\int_{\mathbb{R}^{N}}\left[\nabla w\left(x-R y_{0}\right) \nabla w(x-R y)+w\left(x-R y_{0}\right) w(x-R y)\right] \mathrm{d} x \\
& =\left\langle w\left(\cdot-R y_{0}\right), w(\cdot-R y)\right\rangle \\
& =\left\langle w_{0}^{R}, w_{y}^{R}\right\rangle .
\end{aligned}
$$


Pela Proposição 1.1 e usando (1.40) podemos reescrever a desigualdade em (1.39) da seguinte forma

$$
\begin{aligned}
\frac{J_{\infty}(r u+r v)}{r^{2}}= & \frac{J_{\infty}\left(r \lambda w_{0}^{R}+r(1-\lambda) w_{y}^{R}\right)}{r^{2}} \\
\leq & \lambda^{2}\left(\|w\|^{2}-\int_{\mathbb{R}^{N}} \frac{f(r \lambda w)}{r \lambda w}(w)^{2} \mathrm{~d} x\right) \\
& +(1-\lambda)^{2}\left(\|w\|^{2}-\int_{\mathbb{R}^{N}} \frac{f(r(1-\lambda) w}{r(1-\lambda) w}(w)^{2} \mathrm{~d} x\right) \\
& \quad+2 \lambda(1-\lambda)\left\langle w_{0}^{R}, w_{y}^{R}\right\rangle \\
= & \Phi(\lambda, r)+\Phi(1-\lambda, r)+o_{R}(1) \\
< & S_{0}+o_{R}(1),
\end{aligned}
$$

em que $o_{R}(1) \rightarrow 0$, se $R \rightarrow+\infty$.

Por outro lado, desejamos obter uma estimativa da seguinte forma

$$
\frac{J\left(r \lambda w_{0}^{R}+r(1-\lambda) w_{y}^{R}\right)}{r^{2}}<0,
$$

para todo $r \geq T_{0}, 0 \leq \lambda \leq 1, R \geq R_{0}$ e uniformemente em $y \in \partial B_{2}\left(y_{0}\right)$. Para tanto, observe que para todo $u \in H^{1}\left(\mathbb{R}^{N}\right)$

$$
\begin{aligned}
J(u) & =\|u\|^{2}-\int_{\mathbb{R}^{N}}(1+a(x)) f(u) u \mathrm{~d} x \\
& =\|u\|^{2}-\int_{\mathbb{R}^{N}} f(u) u \mathrm{~d} x-\int_{\mathbb{R}^{N}} a(x) f(u) u \mathrm{~d} x \\
& =J_{\infty}(u)-\int_{\mathbb{R}^{N}} a(x) f(u) u \mathrm{~d} x .
\end{aligned}
$$

Segue por (1.42) que

$$
\begin{aligned}
\frac{J\left(r \lambda w_{0}^{R}+r(1-\lambda) w_{y}^{R}\right)}{r^{2}}= & \frac{J_{\infty}\left(r \lambda w_{0}^{R}+r(1-\lambda) w_{y}^{R}\right)}{r^{2}} \\
& -\frac{1}{r^{2}} \int_{\mathbb{R}^{N}} a(x) f\left(r \lambda w_{0}^{R}+r(1-\lambda) w_{y}^{R}\right)\left(r \lambda w_{0}^{R}+r(1-\lambda) w_{y}^{R}\right) \mathrm{d} x \\
= & \frac{J_{\infty}\left(r \lambda w_{0}^{R}+r(1-\lambda) w_{y}^{R}\right)}{r^{2}} \\
& -\int_{\mathbb{R}^{N}} a(x) \frac{f\left(r \lambda w_{0}^{R}+r(1-\lambda) w_{y}^{R}\right)}{r \lambda w_{0}^{R}+r(1-\lambda) w_{y}^{R}}\left(\lambda w_{0}^{R}+(1-\lambda) w_{y}^{R}\right)^{2} \mathrm{~d} x .
\end{aligned}
$$

Agora vamos estimar esta última integral na equação (1.43), isto é, vamos mostrar que

$$
\int_{\mathbb{R}^{N}}|a(x)| \frac{f\left(r \lambda w_{0}^{R}+r(1-\lambda) w_{y}^{R}\right)}{r \lambda w_{0}^{R}+r(1-\lambda) w_{y}^{R}}\left(\lambda w_{0}^{R}+(1-\lambda) w_{y}^{R}\right)^{2} \mathrm{~d} x=o_{R}(1) .
$$


Podemos utilizar a condição $\left(a_{2}\right),\left(f_{2}\right)$ e o Lema 1.9 verificar $(1.44)$. De fato, note que por $\left(f_{2}\right)$

$$
\begin{aligned}
& \frac{1}{r} \int_{\mathbb{R}^{N}}|a(x)|\left|f\left(r \lambda w_{0}^{R}+r(1-\lambda) w_{y}^{R}\right)\right|\left(\lambda w_{0}^{R}+(1-\lambda) w_{y}^{R}\right) \mathrm{d} x \\
\leq & \frac{C}{r} \int_{\mathbb{R}^{N}}|a(x)|\left\{2^{p_{1}}\left(\left|r \lambda w_{0}^{R}\right|^{p_{1}}+\left|r(1-\lambda) w_{y}^{R}\right|^{p_{1}}\right)+2^{p_{2}}\left(\left|r \lambda w_{0}^{R}\right|^{p_{2}}+\left|r(1-\lambda) w_{y}^{R}\right|^{p_{2}}\right)\right\} \lambda w_{0}^{R} \mathrm{~d} x \\
& +\frac{C}{r} \int_{\mathbb{R}^{N}}|a(x)|\left\{2^{p_{1}}\left(\left|r \lambda w_{0}^{R}\right|^{p_{1}}+\left|r(1-\lambda) w_{y}^{R}\right|^{p_{1}}\right)+2^{p_{2}}\left(\left|r \lambda w_{0}^{R}\right|^{p_{2}}+\left|r(1-\lambda) w_{y}^{R}\right|^{p_{2}}\right)\right\}(1-\lambda) w_{y}^{R} \mathrm{~d} x \\
\leq & C \int_{\mathbb{R}^{N}}|a(x)|\left\{\left(w_{0}^{R}\right)^{p_{1}}+\left(w_{0}^{R}\right)^{p_{2}}+\left(w_{y}^{R}\right)^{p_{1}}+\left(w_{y}^{R}\right)^{p_{2}}\right\}\left(\lambda w_{0}^{R}+(1-\lambda) w_{y}^{R}\right) \mathrm{d} x .
\end{aligned}
$$

Usando agora $\left(a_{2}\right)$ e o Lema 1.9 obtemos

$$
\int_{\mathbb{R}^{N}}|a(x)|\left(w_{0}^{R}\right)^{p_{i}+1} \mathrm{~d} x \leq C \int_{\mathbb{R}^{N}} e^{-k|x|} e^{-\left(p_{i}+1\right)\left|x-R y_{0}\right|} \mathrm{d} x \leq C e^{-k\left|0-R y_{0}\right|}=C e^{-k R}=o_{R}(1),
$$

e também que

$$
\int_{\mathbb{R}^{N}}|a(x)|\left(w_{0}^{R}\right)^{p_{i}} w_{y}^{R} \mathrm{~d} x \leq C \int_{\mathbb{R}^{N}} e^{-k|x|} e^{-p_{i}\left|x-R y_{0}\right|} e^{-|x-R y|} \mathrm{d} x \leq C e^{-\frac{1}{2}(R+2 R+R)}=o_{R}(1),
$$

visto que $C e^{-\frac{1}{2}(R|y|)} \leq C e^{-\frac{1}{2} R}$, pois $1 \leq|y| \leq 3$. Logo, de (1.45), (1.46) e (1.47) verificamos (1.44).

Para concluir o resultado de projeção sobre $\mathcal{N}$, susbtituindo (1.41), (1.44) em (1.43), podemos tomar $R$ suficientemente grande de forma que

$$
\frac{J\left(r \lambda w_{0}^{R}+r(1-\lambda) w_{y}^{R}\right)}{r^{2}} \leq S_{0}+o_{R}(1)<\frac{S_{0}}{3}<0,
$$

para todo $r \in \mathbb{R}$ tal que $T_{0} \leq r, 0 \leq \lambda \leq 1$ e uniformemente em $y \in \partial B_{2}\left(y_{0}\right)$.

Combinando-se (1.4) e (1.48) pode-se garantir que existe um único $r=T_{\lambda, y}^{R} \in\left(0, T_{0}\right)$ tal que $T_{\lambda, y}^{R} z_{\lambda, y}^{R}$ pertence à variedade $\mathcal{N}$. Consequentemente, $\mathcal{N}$ é uma variedade não vazia.

Para provar a continuidade de $T_{\lambda, y}^{R}$, considere a aplicação $h \in C^{1}$ definida por

$$
\begin{aligned}
h: \mathbb{R}^{+} \times H^{1}\left(\mathbb{R}^{N}\right) & \rightarrow \mathbb{R} \\
(t, u) & \mapsto h(t, u)=t\|u\|^{2}-\int_{\mathbb{R}^{N}}(1+a(x)) f(t u) u \mathrm{~d} x .
\end{aligned}
$$

Seja $\left(t_{0}, u_{0}\right)$ tal que $h\left(t_{0}, u_{0}\right)=0$ e $u_{0}>0$. Note que se $t_{0} u_{0} \in \mathcal{N}$, então

$$
t_{0}^{2}\left\|u_{0}\right\|^{2}=\int_{\mathbb{R}^{N}}(1+a(x)) f\left(t_{0} u_{0}\right) t_{0} u_{0} \mathrm{~d} x=\int_{\mathbb{R}^{N}}(1+a(x)) \frac{f\left(t_{0} u_{0}\right)}{t_{0}} t_{0}^{2} u_{0} \mathrm{~d} x .
$$

Assim,

$$
\left\|u_{0}\right\|^{2}=\int_{\mathbb{R}^{N}}(1+a(x)) \frac{f\left(t_{0} u_{0}\right)}{t_{0}} u_{0} \mathrm{~d} x .
$$


Por outro lado, derivando $h$ em relação a $t$ e utilizando (1.49) e $\left(f_{3}\right)$ obtemos

$$
\begin{aligned}
h_{t}^{\prime}\left(t_{0}, u_{0}\right) & =\left\|u_{0}\right\|^{2}-\int_{\mathbb{R}^{N}}(1+a(x)) f^{\prime}\left(t_{0} u_{0}\right) u_{0}^{2} \mathrm{~d} x \\
& =\int_{\mathbb{R}^{N}}(1+a(x))\left(\frac{f\left(t_{0} u_{0}\right)}{t_{0}} u_{0}-f^{\prime}\left(t_{0} u_{0}\right) u_{0}^{2}\right) \mathrm{d} x<0 .
\end{aligned}
$$

Aplicando o Teorema da Função Implícita, a aplicação $u \mapsto t(u)$ é de classe $C^{1}$ onde $t_{0}=t\left(u_{0}\right)$. Pelo que provamos, para cada $R>0, y \in \partial B_{2}\left(y_{0}\right)$ e $\lambda \in[0,1]$ existe um único $T_{\lambda, y}^{R}=t\left(z_{\lambda, y}^{R}\right)$ tal que $t$ é de classe $C^{1}$. Agora, para cada $R>0$ fixado, a função $(\lambda, y) \mapsto z_{\lambda, y}^{R}$ é contínua. Desde que $[0,1] \times \partial B_{2}\left(y_{0}\right)$ é compacto em $\mathbb{R}^{2}$, existe um $\bar{T}(R)=\max _{(\lambda, y) \in[0,1] \times \partial B_{2}\left(y_{0}\right)} T_{\lambda, y}^{R}$ tal que $T_{\lambda, y}^{R} z_{\lambda, y}^{R} \in \mathcal{N}$ e $T_{\lambda, y}^{R} \in[0, \bar{T}(R)]$. Suponha por contradição que $\bar{T}\left(R_{k}\right) \rightarrow+\infty$, se $R_{k} \rightarrow+\infty$. Visto que $\bar{T}\left(R_{k}\right)=\max _{(\lambda, y) \in[0,1] \times \partial B_{2}\left(y_{0}\right)} T_{\lambda, y}^{R_{k}}$, tem-se $\bar{T}\left(R_{k}\right)=T_{\lambda, y}^{R_{k}}$ para algum $(\lambda, y)$. Assim obtemos a continuidade de $T_{\lambda, y}^{R}$ nas variáveis $\lambda, y, R$ e o lema está provado.

\subsection{Estimativas assintóticas}

Precisaremos do Lema II.2 em Bahri e Lions [8] para nos auxiliar nas delicadas e precisas estimativas. Por motivo de completude de informações iremos demonstrar o lema visto que em [8] tal resultado não está claramente enunciado e demonstrado .

Lema 1.11. (Lema II.2 - [8]) Suponha que $\varphi \in C\left(\mathbb{R}^{N}\right) \cap L^{\infty}\left(\mathbb{R}^{N}\right), \psi \in C\left(\mathbb{R}^{N}\right)$ satisfazem para constantes $\alpha, \beta \geq 0$ e $\gamma \in \mathbb{R}$

$$
\varphi(x) e^{\alpha|x|}|x|^{\beta} \rightarrow \gamma ; \text { se }|x| \rightarrow+\infty
$$

$e$

$$
\int_{\mathbb{R}^{N}}|\psi(x)| e^{\alpha|x|}\left(1+|x|^{\beta}\right) \mathrm{d} x<+\infty
$$

Então

$$
\lim _{|\bar{y}| \longrightarrow+\infty}\left[\left(\int_{\mathbb{R}^{N}} \varphi(x+\bar{y}) \psi(x) \mathrm{d} x\right) e^{\alpha|\bar{y}|}|\bar{y}|^{\beta}-\gamma \int_{\mathbb{R}^{N}} e^{-\frac{\alpha\langle x, \bar{y}\rangle}{|\bar{y}|}} \psi(x) \mathrm{d} x\right]=0
$$

Demonstração: A demonstração segue do estudo de vários casos e da aplicação do Teorema da Convergência Dominada de Lebesgue. Primeiramente, seja $|x+\bar{y}| \leq 1$. Note que

$$
|\bar{y}|-|x| \leq|x+\bar{y}| \leq 1 \Longrightarrow|\bar{y}| \leq 1+|x|
$$

Por (1.52) e dado que $\varphi \in L^{\infty}\left(\mathbb{R}^{N}\right)$ existe uma constante $C>0$ tal que

$$
\begin{aligned}
|\varphi(x+\bar{y}) \psi(x)| e^{\alpha|\bar{y}|}|\bar{y}|^{\beta} & \leq C|\psi(x)| e^{\alpha} e^{\alpha|x|}(1+|x|)^{\beta} \\
& =C|\psi(x)| e^{\alpha|x|}\left(1+|x|^{\beta}\right) \in L^{1}\left(\mathbb{R}^{N}\right) .
\end{aligned}
$$


Considere agora $1<|x+\bar{y}| \leq \frac{|\bar{y}|}{2}$. Note que

$$
2|\bar{y}|-2|x| \leq 2|x+\bar{y}| \leq|\bar{y}| \Longrightarrow|\bar{y}| \leq 2|x|
$$

e

$$
|\bar{y}|-|x| \leq|x+\bar{y}| \Longrightarrow-\alpha|x+\bar{y}|+\alpha|\bar{y}| \leq \alpha|x| .
$$

Por (1.51), dado $\epsilon>0$ existe algum $M_{o}>0$ tal que $\forall|x| \geq M_{0}$ tem-se

$$
|\varphi(x)| \leq(\epsilon+\gamma) e^{-\alpha|x|}|x|^{-\beta}=C e^{-\alpha|x|}|x|^{-\beta} .
$$

Combinando as informações de (1.54), (1.55), (1.56) com (1.52) segue que se $1<|x+\bar{y}| \leq \frac{|\bar{y}|}{2}$,

$$
\begin{aligned}
|\varphi(x+\bar{y}) \psi(x)| e^{\alpha|\bar{y}|}|\bar{y}|^{\beta} & \leq C|\psi(x)| e^{-\alpha|x+\bar{y}|}|x+\bar{y}|^{-\beta} e^{\alpha|\bar{y}|}(2|x|)^{\beta} \\
& \leq C|\psi(x)| e^{\alpha|x|}|x|^{\beta} \in L^{1}\left(\mathbb{R}^{N}\right)
\end{aligned}
$$

Finalmente considere o caso $|x+\bar{y}| \geq \frac{|\bar{y}|}{2}$. Usando novamente (1.51) e a desigualdade $-\alpha|x+\bar{y}|+\alpha|\bar{y}| \leq \alpha|x|$ tem-se

$$
\begin{aligned}
|\varphi(x+\bar{y}) \psi(x)| e^{\alpha|\bar{y}|}|\bar{y}|^{\beta} & \leq C|\psi(x)| e^{-\alpha|x+\bar{y}|}|x+\bar{y}|^{-\beta} e^{\alpha|\bar{y}|}(2|x+y|)^{\beta} \\
& \leq C|\psi(x)| e^{\alpha|x|}|x|^{\beta} \in L^{1}\left(\mathbb{R}^{N}\right) .
\end{aligned}
$$

Para cada $x \in \mathbb{R}^{N}$ fixado, segue de (1.51) que

$$
\lim _{|\bar{y}| \longrightarrow+\infty} \varphi(x+\bar{y}) \psi(x) e^{\alpha|\bar{y}|}|\bar{y}|^{\beta}=\lim _{|x+\bar{y}| \longrightarrow+\infty} \varphi(x+\bar{y}) \psi(x) e^{\alpha|x+\bar{y}|}|x+\bar{y}|^{\beta}=\gamma \psi(x) .
$$

Com os resultados obtidos em (1.54), (1.57), (1.58) e (1.59) podemos aplicar o Teorema da Convergência Dominada de Lebesgue para obter

$$
\lim _{|\bar{y}| \longrightarrow+\infty}\left(\int_{\mathbb{R}^{N}} \varphi(x+\bar{y}) \psi(x) \mathrm{d} x\right) e^{\alpha|\bar{y}|}|\bar{y}|^{\beta}=\gamma \int_{\mathbb{R}^{N}} \psi(x) \mathrm{d} x .
$$

Por outro lado, observe que se $\langle x, \bar{y}\rangle \geq 0$, então

$$
\left|\gamma e^{-\frac{\alpha\langle x, \bar{y}\rangle}{|\bar{y}|}} \psi(x)\right| \leq|\gamma||\psi(x)|,
$$

e $\gamma \psi \in L^{1}\left(\mathbb{R}^{N}\right)$ uma vez que

$$
\int_{\mathbb{R}^{N}}|\psi(x)| \mathrm{d} x \leq \int_{\mathbb{R}^{N}}|\psi(x)| e^{\alpha|x|}\left(1+|x|^{\beta}\right) \mathrm{d} x<+\infty .
$$

Se $\langle x, \bar{y}\rangle \leq 0$, então

$$
0 \leq-\langle x, \bar{y}\rangle=\langle-x, \bar{y}\rangle \leq|x||\bar{y}|
$$


implica que

$$
\left|\gamma e^{-\frac{\alpha\langle x, y\rangle}{|y|}} \psi(x)\right| \leq|\gamma| e^{\alpha|x|}|\psi(x)| \in L^{1}\left(\mathbb{R}^{N}\right),
$$

por (1.52). Para cada $x \in \mathbb{R}^{N}$ fixado, temos ainda

$$
\lim _{|\bar{y}| \rightarrow+\infty} \gamma e^{-\frac{\alpha\langle x, \bar{y}\rangle}{|\bar{y}|}} \psi(x)=\gamma e^{0} \psi(x) .
$$

Com os resultados obtidos em (1.61), (1.62) e (1.63) podemos aplicar o Teorema da Convergência Dominada de Lebesgue para obter

$$
\lim _{|\bar{y}| \rightarrow+\infty} \int_{\mathbb{R}^{N}} \gamma e^{-\frac{\alpha\langle x, \bar{y}\rangle}{|\bar{y}|}} \psi(x) \mathrm{d} x=\gamma \int_{\mathbb{R}^{N}} \psi(x) \mathrm{d} x .
$$

Portanto, de (1.60) e (1.64) o lema está demonstrado.

Um resultado encontrado em [37] que iremos utilizar para obter o decaimento exponencial exato para a solução ground state do problema limite $\left(P_{\infty}\right)$, a saber

Lema 1.12. [Proposição 6.1 em [37]] Sejam $N>1, \rho \geq 0$ e W $\in C^{1}((\rho, \infty), \mathbb{R})$. Se

$$
\lim _{s \rightarrow \infty} W(s)>0
$$

e para algum $\beta>0$ tem-se

$$
\lim _{s \rightarrow \infty} W^{\prime}(s) s^{1+\beta}=0
$$

então existe uma função radial não negativa $v: \mathbb{R}^{N} \backslash B_{\rho}(0) \rightarrow \mathbb{R}$ tal que

$$
-\Delta v+W v=0 \quad \text { em } \mathbb{R}^{N} \backslash B_{\rho}(0)
$$

e, para alguma constante $\rho_{0} \in(\rho, \infty)$,

$$
\lim _{|x| \rightarrow \infty} v(x)|x|^{\frac{N-1}{2}} \exp \int_{\rho_{0}}^{|x|} \sqrt{W}=1 .
$$

Seguindo idéias encontradas em [38], mostraremos que a solução ground state positiva do problema limite $\left(P_{\infty}\right)$ tem decaimento exponencial exato. Tal demonstração é diferente daquela encontrada no clássico artigo de Gidas, Ni e Niremberg [25] em que os autores demonstraram o decaimento exponencial utilizando função de Green, estimativas interiores e funções de Bessel.

Lema 1.13. Seja u solução positiva radial ground state do problema

$$
-\Delta u+u=f(u) \quad \text { em } \mathbb{R}^{N}
$$

então u satisfaz 


$$
\lim _{|x| \rightarrow \infty} u(x)|x|^{\frac{N-1}{2}} e^{|x|} \in(0, \infty) .
$$

Demonstração: Inicialmente, visto que $u(x) \rightarrow 0$, se $|x| \rightarrow \infty$, segue por $\left(f_{2}\right)$ que

$$
\lim _{|x| \rightarrow \infty} \frac{f(u(x))}{u(x)}=0 .
$$

Logo, existe um $\rho \in \mathbb{R}$ tal que para todo $x \in \mathbb{R}^{N}$ e $|x| \geq \rho$ temos

$$
\frac{f(u(x))}{u(x)} \leq \frac{3}{4} .
$$

Note que se $x \in \mathbb{R}^{N} \backslash B_{\rho}(0)$,

$$
-\Delta u(x)+u(x)=f(u(x))=\frac{f(u(x))}{u(x)} u(x) \leq \frac{3 u(x)}{4} \quad \text { em } \mathbb{R}^{N} \backslash B_{\rho}(0),
$$

implicando

$$
-\Delta u(x)+\frac{1}{4} u(x) \leq \frac{3 u(x)}{4}-\frac{3 u(x)}{4}=0 \quad \text { em } \mathbb{R}^{N} \backslash B_{\rho}(0) .
$$

Considere uma função radial não negativa $v \in C^{2}\left(\mathbb{R}^{N} \backslash B_{\rho}(0), \mathbb{R}\right)$ satisfazendo

$$
\left\{\begin{aligned}
-\Delta v+\frac{1}{4} v & =0, \quad \text { se } x \in \mathbb{R}^{N} \backslash B_{\rho}(0), \\
v(x) & =u(x), \quad \text { se } x \in \partial B_{\rho}(0) \\
\lim _{|x| \rightarrow \infty} v(x) & =0
\end{aligned}\right.
$$

Seja $W_{0}(x)=\frac{1}{4}$ e note que $W=W_{0}$ no Lema 1.12, juntamente com (1.65) e (1.66) existe uma constante $C>0$ tal que

$$
0<v(x) \leq C|x|^{-\frac{N-1}{2}} e^{-\frac{|x|}{2}}, \text { para todo } x \in \mathbb{R}^{N} \backslash B_{\rho}(0) .
$$

Defina a função $z:=u-v$. Segue por (1.68) e (1.69)

$$
-\Delta z+\frac{1}{4} z=-\Delta u+\frac{1}{4} u+\Delta v-\frac{1}{4} v \leq 0 \quad \text { em } \quad \mathbb{R}^{N} \backslash B_{\rho}(0)
$$

e

$$
z=0 \text { sobre } \partial B_{\rho}(0)
$$

Ademais, temos que

$$
\lim _{|x| \rightarrow \infty} z(x)=0
$$

Considere $z=z^{+}-z^{-}$. Por (1.70) segue

$$
\left\|z^{+}\right\|^{2}=\int_{\mathbb{R}^{N} \backslash B_{\rho}(0)}\left(\left|\nabla z^{+}\right|^{2}+\frac{1}{4}\left|z^{+}\right|\right) \mathrm{d} x \leq 0,
$$

logo $z \leq 0$ em $\mathbb{R}^{N} \backslash B_{\rho}(0)$. Assim 


$$
0<u(x) \leq v(x) \leq C|x|^{-\frac{N-1}{2}} e^{-\frac{|x|}{2}} \text { em } \mathbb{R}^{N} \backslash B_{\rho}(0) .
$$

Combinando a hipótese de crescimento $\left(f_{2}\right)$ e (1.72) existe uma constante $C>0$ tal que

$$
\left|\frac{f(u)}{u}\right| \leq C\left(e^{-\frac{p_{1}-1}{2}|x|}+e^{-\frac{p_{2}-1}{2}|x|}\right) .
$$

Considere $W_{1}(x)=1-C\left(e^{-\frac{p_{1}-1}{2}|x|}+e^{-\frac{p_{2}-1}{2}|x|}\right)$ e note que $W_{1}$ satisfaz o Lema 1.12 . Aplicando-o mais uma vez e usando (1.73) temos

$$
\begin{aligned}
-\Delta u+W_{1} u & =-\Delta u+u-C\left(e^{-\frac{p_{1}-1}{2}|x|}+e^{-\frac{p_{2}-1}{2}|x|}\right) u \\
& =\frac{f(u)}{u} u-C\left(e^{-\frac{p_{1}-1}{2}|x|}+e^{-\frac{p_{2}-1}{2}|x|}\right) u \\
& =\left[\frac{f(u)}{u}-C\left(e^{-\frac{p_{1}-1}{2}|x|}+e^{-\frac{p_{2}-1}{2}|x|}\right)\right] u \\
& \leq 0 \text { em } \mathbb{R}^{N} \backslash B_{\rho}(0) .
\end{aligned}
$$

Consequentemente,

$$
-\Delta u+u \geq 0 \geq-\Delta u+W_{1} u \text { em } \mathbb{R}^{N} \backslash B_{\rho}(0) .
$$

Defina as funções radiais não negativas $\underline{u}, \bar{u} \in C^{2}\left(\mathbb{R}^{N} \backslash B_{\rho}(0), \mathbb{R}\right)$ satisfazendo

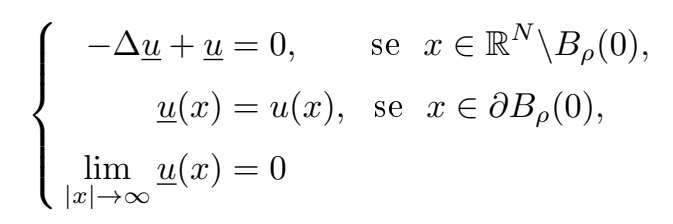

e

$$
\left\{\begin{array}{rlrl}
-\Delta \bar{u}+W_{1} \bar{u} & =0, & & \text { se } x \in \mathbb{R}^{N} \backslash B_{\rho}(0), \\
\bar{u}(x) & =u(x), & \text { se } x \in \partial B_{\rho}(0), \\
\lim _{|x| \rightarrow \infty} \bar{u}(x) & =0 . & &
\end{array}\right.
$$

Observe que

$$
-\Delta(\underline{u}-u)+(\underline{u}-u)=(-\Delta \underline{u}+\underline{u})+(\Delta u-u) \leq 0,
$$

isto é,

$$
-\Delta \underline{u}+\underline{u} \leq-\Delta u+u \text { em } \mathbb{R}^{N} \backslash B_{\rho}(0)
$$

e

$$
\underline{u}=u \text { sobre } \partial B_{\rho}(0) .
$$

Analogamente, temos

$$
-\Delta(\bar{u}-u)+W_{1}(\bar{u}-u)=\left(-\Delta \bar{u}+W_{1} \bar{u}\right)+\left(\Delta u-W_{1} u\right) \geq 0,
$$

isto é,

$$
-\Delta u+W_{1} u \leq-\Delta \bar{u}+W_{1} \bar{u} \text { em } \mathbb{R}^{N} \backslash B_{\rho}(0)
$$


e

$$
\bar{u}=u \text { sobre } \partial B_{\rho}(0) .
$$

Com estas duas observações segue analogamente como feito em (1.71) que

$$
\underline{u} \leq u \leq \bar{u} \text { em } \mathbb{R}^{N} \backslash B_{\rho}(0)
$$

Utilizando (1.77) e o Lema 1.12 segue que

$$
\begin{aligned}
0<\lim _{|x| \rightarrow \infty} \underline{u}(x)|x|^{\frac{N-1}{2}} e^{|x|} & \leq \liminf _{|x| \rightarrow \infty} u(x)|x|^{\frac{N-1}{2}} e^{|x|} \\
& \leq \limsup _{|x| \rightarrow \infty} u(x)|x|^{\frac{N-1}{2}} e^{|x|} \\
& \leq \lim _{|x| \rightarrow \infty} \bar{u}(x)|x|^{\frac{N-1}{2}} e^{|x|}<\infty
\end{aligned}
$$

A primeira desigualdade estrita em (1.78) segue do Lema 1.12 e a última desigualdade é finita, pois utilizando o Lema 1.12 e (1.76) obtemos

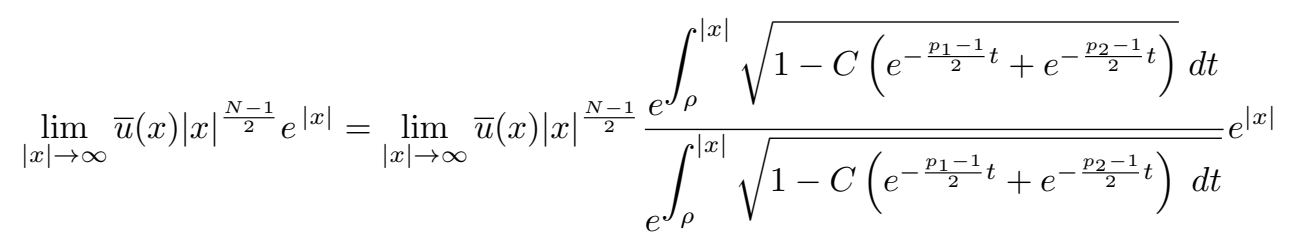

$$
\begin{aligned}
& =\lim _{|x| \rightarrow \infty} \bar{u}(x)|x|^{\frac{N-1}{2}} e^{\int_{\rho}^{|x|} \sqrt{1-C\left(e^{-\frac{p_{1}-1}{2} t}+e^{-\frac{p_{2}-1}{2} t}\right)} d t} \lim _{|x| \rightarrow \infty} \frac{e^{|x|}}{\int_{\rho}^{|x|} \sqrt{1-C\left(e^{-\frac{p_{1}-1}{2} t}+e^{-\frac{p_{2}-1}{2} t}\right)} d t}<\infty .
\end{aligned}
$$

O próximo passo é mostrar que o $\lim _{|x| \rightarrow \infty} u(x)|x|^{\frac{N-1}{2}} e^{|x|}$ existe. De fato, note que se mostrarmos que a função $\frac{u}{\underline{u}}$ é não decrescente, usando (1.77) tal função seria limitada, pois $1 \leq \frac{u}{\underline{u}} \leq \frac{\bar{u}}{\underline{u}} \mathrm{e}$

$$
\begin{aligned}
& \lim _{|x| \rightarrow \infty} \frac{\bar{u}(x)}{\underline{u}(x)}
\end{aligned}
$$

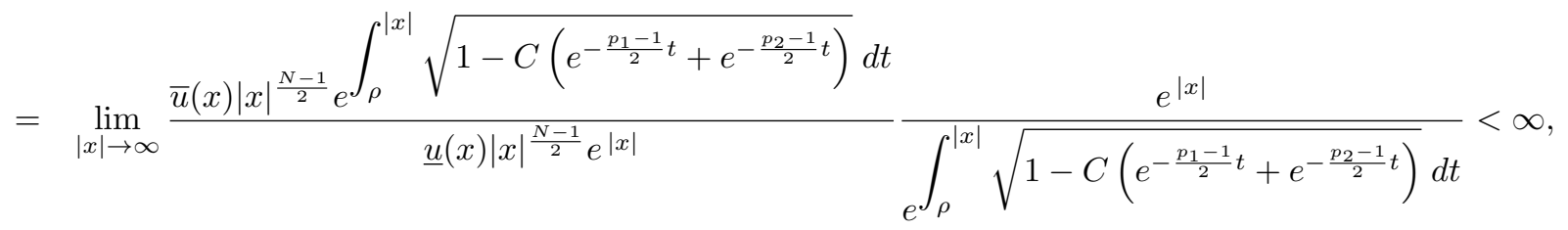

por $(1.75),(1.76)$ e o Lema 1.12. Assim concluiríamos que $\frac{u}{\underline{u}}$ tem limite finito se $|x| \rightarrow+\infty$ e que

$$
\lim _{|x| \rightarrow \infty} u(x)|x|^{\frac{N-1}{2}} e^{|x|}=\lim _{|x| \rightarrow \infty} \frac{u(x)}{\underline{u}(x)} \lim _{|x| \rightarrow \infty} \underline{u}(x)|x|^{\frac{N-1}{2}} e^{|x|} \in(0, \infty) .
$$

Finalmente, vamos mostrar que $\frac{u}{\underline{u}}$ é não decrescente. Considere $r, s \in(\rho, \infty)$ tal que $r \leq s$. Desde 
que $u$ e $\underline{u}$ são ambas funções radiais, defina o seguinte operador

$$
L u=-(-\Delta u+u)=\Delta u-u=u^{\prime \prime}+\frac{N-1}{r} u^{\prime}-u .
$$

Segue da definição do operador L e de (1.75) que

$$
L \underline{u}=\underline{u}^{\prime \prime}+\frac{N-1}{r} \underline{u}^{\prime}-\underline{u} .
$$

Note que $-\Delta(\underline{u}-u)+(\underline{u}-u)=-f(u)<0, \log$, como feito em (1.71), temos que $\underline{u}(x) \leq u(x)$ para todo $|x| \in(\rho, \infty)$.

Agora considere a função $v=\frac{u}{u}$ e $g(r)=\frac{N-1}{r}$. Observe que

$$
L \underline{u}=L(v u)=u v^{\prime \prime}+\left(2 u^{\prime}+g u\right) v^{\prime}+L(u) v=0 .
$$

Decorre que

$$
v^{\prime \prime}+\left(2 \frac{u^{\prime}}{u}+g\right) v^{\prime}+\frac{1}{u} L(u) v ; \quad r \in(\rho, \infty), \quad v(\rho)=1 .
$$

O problema de valor inicial em (1.79) tem solução única desde que $\frac{1}{u} L(u)<0$ e $2 \frac{u^{\prime}}{u}+g$ é contínua em $r \in(\rho, \infty)$.

Por outro lado, note que temos

$$
L(\underline{u}-u)=0-(-f(u))=f(u)>0, \text { para } r \in(\rho, \infty),
$$

e

$$
\underline{u}-u=0 \text { sobre } \partial B_{\rho}(0) .
$$

Aplicando o Princípio do Máximo e Lema de Hopf , respectivamente, temos

$$
\underline{u}-u<0 \text { para } r>\rho \text { e } \frac{\partial(\underline{u}-u)}{\partial r}<0 \text { se } r=\rho,
$$

que implica

$$
\underline{u}^{\prime}(\rho)<u^{\prime}(\rho)<0
$$

Consequentemente,

$$
v^{\prime}(\rho)=\frac{\underline{u}^{\prime}(\rho) u(\rho)-\underline{u}(\rho) u^{\prime}(\rho)}{u^{2}(\rho)}<\frac{u^{\prime}(\rho)[u(\rho)-\underline{u}(\rho)]}{u^{2}(\rho)}=0 .
$$

Desde que $v(r)$ é continuamente diferenciável em $(\rho, \infty)$, derivando $v$ temos

$$
v^{\prime}(r)=\frac{\underline{u}^{\prime}(r) u(r)-\underline{u}(r) u^{\prime}(r)}{u^{2}(r)} .
$$

Supondo $v^{\prime}(r) \geq 0$ segue que

$$
\frac{\underline{u}^{\prime}(r)}{\underline{u}(r)} \geq \frac{u^{\prime}(r)}{u(r)}
$$


ou seja,

$$
(\ln \underline{u}(r))^{\prime} \geq(\ln u(r))^{\prime} .
$$

Como a função $\ln (x)$ é uma função crescente, isto equivale a

$$
\underline{u}(r) \geq u(r) \text {. Contradição com (1.80). }
$$

Assim temos que $v^{\prime}(r)<0$ para todo $r>\rho$ e, portanto, implicando que $\frac{u}{\underline{u}}$ é não decrescente como desejávamos mostrar.

No próximo resultado iremos enunciar e provar duas propriedades das funções $F$ e $f$ que são consequências de $\left(f_{3}\right)$ e que foi observado inicialmente no trabalho [24]. Tais propriedades são válidas sem exigir que $f$ seja de classe $C^{3}$ como nos trabalhos em [1, 18].

Lema 1.14. Assuma $\left(f_{3}\right)$. Para todo $u, v>0$ tem-se

$$
F(u+v) \geq F(u)+f(u) v,
$$

e para todo $\rho>0$ existe uma constante $C_{\rho}>0$ tal que, para todo $0 \leq u, v \leq \rho$ e $\mu$ satisfazendo $0<1+\mu<p_{1}$, tem-se

$$
F(u+v)-F(u)-F(v) \geq f(u) v+f(v) u-C_{\rho} u^{1+\frac{\mu}{2}} v^{1+\frac{\mu}{2}} .
$$

Demonstração: Primeiramente, como consequência de $\left(f_{3}\right)$ temos que a função $u \mapsto f(u)$ é crescente e isto implica que

$$
F(u+v)-F(u)=\int_{u}^{u+v} f(t) \mathrm{d} t \geq f(u) v .
$$

Por outro lado, se $u=0$ ou $v=0$, a segunda propriedade é satisfeita. Entretanto, para $0<v \leq u$, segue de (1.81) que

$$
\begin{aligned}
F(u+v)-F(u)-F(v)-f(u) v-f(v) u & \geq-F(v)-f(v) u \\
& =-\int_{0}^{v} \frac{f(t)}{t^{1+\nu}} t^{1+\mu} \mathrm{d} t-\frac{f(v)}{v^{1+\nu}} u v^{1+\mu} \\
& \geq-\frac{\bar{C}_{\rho}}{2+\mu} v^{2+\mu}-\bar{C}_{\rho} u v^{1+\mu} \\
& =\bar{C}_{\rho}(u v)^{1+\frac{\mu}{2}}\left\{-\frac{1}{2+\mu} \frac{v^{1+\frac{\mu}{2}}}{u^{1+\frac{\mu}{2}}}-\frac{v^{\frac{\mu}{2}}}{u^{\frac{\mu}{2}}}\right\} \\
& \geq \bar{C}_{\rho}(u v)^{1+\frac{\mu}{2}}\left\{-\frac{1}{2+\mu} \frac{v^{1+\frac{\mu}{2}}}{u^{1+\frac{\mu}{2}}}-\frac{u^{\frac{\mu}{2}}}{u^{\frac{\mu}{2}}}\right\} \\
& \geq \bar{C}_{\rho}(u v)^{1+\frac{\mu}{2}}\left\{-\frac{1}{2+\mu}-1\right\} \\
& \geq \bar{C}_{\rho}(u v)^{1+\frac{\mu}{2}}\left\{-\frac{1}{2}-1\right\} \\
& =-\frac{3}{2} \bar{C}_{\rho} u^{1+\frac{\mu}{2}} v^{1+\frac{\mu}{2}}
\end{aligned}
$$


onde $\bar{C}_{\rho}:=\sup _{0<u<\rho} \frac{f(u)}{u^{1+\mu}}<\infty$ e para todo $\mu$ tal que $0<1+\mu<p_{1}$.

Como (1.81) e (1.82) são simétricas em relação a $u$ e $v$, a mesma estimativa se mantém para $0<u \leq v$ e, assim, concluímos a demonstração.

Agora iremos iniciar as precisas estimativas. Para isso, começamos definindo para $R>0$, $y \in \partial B_{2}\left(y_{0}\right),\left\|y_{0}\right\|=1$ e $y_{0} \in \mathbb{R}^{N}$ fixado, a quantidade

$$
\varepsilon_{R}:=\int_{\mathbb{R}^{N}} f\left(w\left(x-R y_{0}\right)\right) w(x-R y) \mathrm{d} x
$$

onde estamos trabalhando com $w$ solução positiva radial do problema $\left(P_{\infty}\right)$. Para mais informações sobre (1.83), veja os trabalhos $[1,8,18]$ e suas referências.

Nos dois próximos lemas iremos estimar a quantidade $\varepsilon_{R}$, isto é, mostrar o decaimento exato da quantidade $\varepsilon_{R}$ como consequência dos Lemas 1.11 e 1.13 e das hipóteses $\left(f_{2}\right)$ e $\left(f_{3}\right)$.

Lema 1.15. Assuma $\left(f_{2}\right)$ e considere $y \in \partial B_{2}\left(y_{0}\right)$ com $y_{0} \in \mathbb{R}^{N} ;\left\|y_{0}\right\|=1$. Então existe uma constante $C_{0}>0$ tal que

$$
\lim _{R \longrightarrow+\infty} \varepsilon_{R}(2 R)^{\frac{N-1}{2}} e^{2 R}=C_{0} .
$$

Demonstração: Para demonstrarmos este lema iremos utilizar o Lema $1.11 \operatorname{com} \varphi=\omega, \psi=f(\omega)$ e $\bar{y}=-R\left(y_{0}-y\right)$ e ainda $\alpha=1$ e $\beta=\frac{N-1}{2}$.

Pelo Lema 1.13 , se $\omega$ é a solução positiva radial do problema limite $\left(P_{\infty}\right)$, então $\omega$ tem o comportamento assintótico

$$
\lim _{|x| \rightarrow \infty} \omega(|x|)|x|^{\frac{N-1}{2}} e^{|x|} \in(0, \infty) .
$$

Portanto, a primeira hipótese do Lema 1.11, isto é, (1.51) está verificada em (1.85).

Por outro lado, usando $\left(f_{2}\right)$ e (1.85) existe algum $R_{1}>0$ tal que, para todo $|x|>R_{1}$,

$$
\begin{aligned}
\psi=f(\omega) & \leq C\left(|\omega|^{p_{1}}+|\omega|^{p_{2}}\right) \\
& \leq C\left(|x|^{-p_{1} \frac{N-1}{2}} e^{-p_{1}|x|}+|x|^{-p_{2} \frac{N-1}{2}} e^{-p_{2}|x|}\right) .
\end{aligned}
$$


Decorre de (1.86) que

$$
\begin{aligned}
& \int_{\mathbb{R}^{N}} f(\omega) e^{|x|}(1+|x|)^{\frac{N-1}{2}} \mathrm{~d} x \\
& =\int_{B_{R_{1}}(0)} f(\omega) e^{|x|}(1+|x|)^{\frac{N-1}{2}} \mathrm{~d} x+\int_{\mathbb{R}^{N} \backslash B_{R_{1}}(0)} f(\omega) e^{|x|}(1+|x|)^{\frac{N-1}{2}} \mathrm{~d} x \\
& \leq C \mu\left(B_{R_{1}}(0)\right)+\int_{\mathbb{R}^{N} \backslash B_{R_{1}}(0)} f(\omega) e^{|x|}(1+|x|)^{\frac{N-1}{2}} \mathrm{~d} x \\
& \leq C+\int_{\mathbb{R}^{N} \backslash B_{R_{1}}(0)} C\left(|x|^{-p_{1} \frac{N-1}{2}} e^{-p_{1}|x|}+|x|^{-p_{2} \frac{N-1}{2}} e^{-p_{2}|x|}\right) e^{|x|}(1+|x|)^{\frac{N-1}{2}} \mathrm{~d} x \\
& =C+C \int_{\mathbb{R}^{N} \backslash B_{R_{1}}(0)}\left(|x|^{-p_{1} \frac{N-1}{2}} e^{\left(1-p_{1}\right)|x|}+|x|^{-p_{2} \frac{N-1}{2}} e^{\left(1-p_{2}\right)|x|}\right)(1+|x|)^{\frac{N-1}{2}} \mathrm{~d} x \\
& \leq C+C \int_{\mathbb{R}^{N} \backslash B_{R_{1}}(0)}\left(R^{-p_{1} \frac{N-1}{2}} e^{\left(1-p_{1}\right)|x|}+R^{-p_{2} \frac{N-1}{2}} e^{\left(1-p_{2}\right)|x|}\right)(1+|x|)^{\frac{N-1}{2}} \mathrm{~d} x \\
& =C+C R^{-p_{1} \frac{N-1}{2}} \int_{\mathbb{R}^{N} \backslash B_{R_{1}}(0)} e^{\left(1-p_{1}\right)|x|}(1+|x|)^{\frac{N-1}{2}} \mathrm{~d} x \\
& +C R^{-p_{2} \frac{N-1}{2}} \int_{\mathbb{R}^{N} \backslash B_{R_{1}}(0)} e^{\left(1-p_{2}\right)|x|}(1+|x|)^{\frac{N-1}{2}} \mathrm{~d} x<+\infty,
\end{aligned}
$$

pois $1-p_{i}<0$ para $i \in\{1,2\}$. Desta forma verificamos a segunda hipótese do Lema 1.11, isto é, (1.52) está verificada. Podemos então aplicar o Lema 1.11 que assegura

$$
\lim _{R \rightarrow+\infty} \varepsilon_{R}(2 R)^{\frac{N-1}{2}} e^{2 R}=C_{0}>0 .
$$

No lema seguinte apresentaremos uma estimativa inferior para a quantidade $\varepsilon_{R}$, se $s=t=1$.

Lema 1.16. Existe uma constante $\bar{C}_{0}>0$ tal que para todo $t, s \geq \frac{1}{2}, y \in \partial B_{2}\left(y_{0}\right)$ com $y_{0} \in \mathbb{R}^{N}$ e $R$ suficientemente grande,

$$
\int_{\mathbb{R}^{N}} f\left(s w\left(x-R y_{0}\right)\right) t w(x-R y) \mathrm{d} x \geq \bar{C}_{0}(2 R)^{-\frac{N-1}{2}} e^{-2 R} .
$$

Demonstração: Inicialmente, como $s, t \geq \frac{1}{2}$ e usando a hipótese $\left(f_{3}\right)$ segue que

$$
\frac{f\left(s w_{0}\right)}{s w_{0}} \geq \frac{f\left(\frac{1}{2} w_{0}\right)}{\frac{1}{2} w_{0}} .
$$

Consequentemente,

$$
\begin{aligned}
\int_{R^{N}} f\left(s w_{0}\right) t w_{y} \mathrm{~d} x & =s t \int_{R^{N}} \frac{f\left(s w_{0}\right)}{s w_{0}} w_{0} w_{y} \mathrm{~d} x \\
& \geq \frac{1}{2} \frac{1}{2} \int_{R^{N}} \frac{f\left(\frac{1}{2} w_{0}\right)}{\frac{1}{2} w_{0}} w_{0} w_{y} \mathrm{~d} x \\
& \geq \frac{1}{4} \int_{B_{1}\left(R y_{0}\right)} \frac{f\left(\frac{1}{2} w_{0}\right)}{\frac{1}{2} w_{0}} w_{0} w_{y} \mathrm{~d} x .
\end{aligned}
$$


Como $\frac{f(s)}{s}$, para $s>0$, é contínua em $B_{1}(0)$, então $\frac{f\left(\frac{1}{2} w_{0}\right)}{\frac{1}{2} w_{0}}$ atinge um mínimo. Assim, fazendo mudança de variáveis

$$
\begin{aligned}
\int_{R^{N}} f\left(s w_{0}\right) t w_{y} \mathrm{~d} x & \geq \frac{1}{4} \int_{B_{1}\left(R y_{0}\right)} \frac{f\left(\frac{1}{2} w_{0}\right)}{\frac{1}{2} w_{0}} w_{0} w_{y} \mathrm{~d} x \\
& \geq \frac{1}{4} \int_{B_{1}(0)} \frac{f\left(\frac{1}{2} w(x)\right)}{\frac{1}{2} w(x)} w(x) w\left(x-R\left(y-y_{0}\right)\right) \mathrm{d} x \\
& \geq \frac{1}{4} \min _{B_{1}(0)} \frac{f\left(\frac{1}{2} w(x)\right)}{\frac{1}{2} w(x)} \int_{B_{1}(0)} w(x) w\left(x-R\left(y-y_{0}\right)\right) \mathrm{d} x \\
& \geq \frac{1}{4} C(2 R)^{-\frac{N-1}{2}} e^{-2 R}=\bar{C}_{0}(2 R)^{-\frac{N-1}{2}} e^{-2 R},
\end{aligned}
$$

pois, usando a desigualdade triangular e como $x \in B_{1}(0)$, para todo $R \geq 1$, segue que

$$
1+\left|x-R\left(y-y_{0}\right)\right| \leq 1+|x|+R\left|y-y_{0}\right| \leq R+R+2 R=4 R
$$

e combinando (1.88) e (1.24) resulta que

$$
\begin{aligned}
w\left(x-R\left(y-y_{0}\right)\right) & \geq C_{1}\left(1+\left|x-R\left(y-y_{0}\right)\right|\right)^{-\frac{N-1}{2}} e^{-\mid x-R\left(y-y_{0} \mid\right.} \\
& \geq C_{1}(4 R)^{-\frac{N-1}{2}} e^{-|x|} e^{-\mid R\left(y-y_{0} \mid\right.} \\
& \geq C(2 R)^{-\frac{N-1}{2}} e^{-1} e^{-2 R} \\
& =C(2 R)^{-\frac{N-1}{2}} e^{-2 R} .
\end{aligned}
$$

Assim, o lema está demonstrado.

Para nossas precisas estimativas de energia precisaremos utilizar os próximos dois resultados técnicos.

Corolário 1.1. Existe uma constante positiva $C$ tal que

$$
\left|\int_{R^{N}}\left(s f\left(w_{0}^{R}\right)-f\left(s w_{0}^{R}\right)\right) w_{y}^{R} \mathrm{~d} x\right| \leq C|s-1| O\left(\varepsilon_{R}\right),
$$

uniformemente para $y \in \partial B_{2}\left(y_{0}\right), s \in[0, d] ; d>1$ e $R$ suficientemente grande.

Demonstração: Defina $\psi(s):=s f(u)-f(s u)$ para $u$ no conjunto dos números reais. Pela regularidade da $f$ podemos usar o Teorema do Valor Médio para $\psi$ o qual garante a existência de um $\xi$ entre $s$ e 1 , sem perda de generalidade, considere $s>1$ tal que

$$
|\psi(s)-\psi(1)| \leq\left|\psi^{\prime}(\xi)\right||s-1|
$$


Derivando $\psi$ em relação a $s$ e usando a hipótese $\left(f_{2}\right)$ tem-se

$$
\begin{aligned}
\left|\psi^{\prime}(s)\right| & =\left|f(u)-f^{\prime}(s u) u\right| \\
& \leq|f(u)|+\left|f^{\prime}(s u)\right||u| \\
& \leq|f(u)|+C\left(|s u|^{p_{1}-1}|u|+|s u|^{p_{2}-1}|u|\right) \\
& \leq|f(u)|+C \max \left\{|s|^{p_{1}-1},|s|^{p_{2}-1}\right\}\left(|u|^{p_{1}}+|u|^{p_{2}}\right) \\
& \leq|f(u)|+C\left(|u|^{p_{1}}+|u|^{p_{2}}\right) .
\end{aligned}
$$

Combinando as desigualdades em (1.89) e (1.90) segue que

$$
|\psi(s)|=|\psi(s)-\psi(1)| \leq\left(|f(u)|+C\left(|u|^{p_{1}}+|u|^{p_{2}}\right)\right)|s-1| .
$$

Tomando $u=w_{0}^{R}:=w\left(x-R y_{0}\right)$, segue que

$$
\begin{aligned}
\left|\int_{R^{N}}\left(s f\left(w_{0}^{R}\right)-f\left(s w_{0}^{R}\right)\right) w_{y}^{R} \mathrm{~d} x\right| & =\left|\int_{R^{N}} \psi(s) w_{y}^{R} \mathrm{~d} x\right| \\
& \leq \int_{R^{N}}\left\{\left|f\left(w_{0}^{R}\right)\right|+C\left(\left|w_{0}^{R}\right|^{p_{1}}+\left|w_{0}^{R}\right|^{p_{2}}\right)\right\} w_{y}^{R}|s-1| \mathrm{d} x \\
& =|s-1|\left\{\int_{R^{N}}\left|f\left(w_{0}^{R}\right) w_{y}^{R}\right| \mathrm{d} x+C \int_{R^{N}}\left(\left|w_{0}^{R}\right|^{p_{1}} w_{y}^{R}+\left|w_{0}^{R}\right|^{p_{2}} w_{y}^{R}\right) \mathrm{d} x\right\} \\
& \leq|s-1|\left\{\varepsilon_{R}+O\left(\varepsilon_{R}\right)\right\},
\end{aligned}
$$

pois para $i \in\{1,2\}$, usando (1.24), o Lema 1.9 e argumentando como no Lema 1.15 temos

$$
\int_{R^{N}}\left|w_{0}^{R}\right|^{p_{i}} w_{y}^{R} \mathrm{~d} x=\int_{R^{N}}|w(z)|^{p_{i}} w\left(z-R\left(y-y_{0}\right)\right) \mathrm{d} z \leq O\left(\varepsilon_{R}\right) .
$$

Assim concluímos a demonstração do corolário.

Lema 1.17. Considere $\lambda=\frac{1}{2}$. Se $R \rightarrow+\infty$, então tem-se que

$$
T_{\frac{1}{2}, y}^{R} \rightarrow 2
$$

uniformemente em $y \in \partial B_{2}\left(y_{0}\right)$.

Demonstração: Considerando $z_{\lambda, y}^{R}$ definido em (1.25) e fazendo $\lambda=\frac{1}{2}$ temos

$$
z_{\frac{1}{2}, y}^{R}=\frac{1}{2} w\left(x-R y_{0}\right)+\frac{1}{2} w(x-R y)=\frac{1}{2} w_{0}^{R}+\frac{1}{2} w_{y}^{R}
$$


Usando o funcional $J$ dado em (1.5) segue que

$$
\begin{aligned}
J\left(2 z_{\frac{1}{2}, y}^{R}\right)=J\left(w_{0}^{R}+w_{y}^{R}\right)= & \left\|w_{0}^{R}+w_{y}^{R}\right\|^{2}-\int_{\mathbb{R}^{N}}(1+a(x)) f\left(w_{0}^{R}+w_{y}^{R}\right)\left(w_{0}^{R}+w_{y}^{R}\right) \mathrm{d} x \\
= & \left\|w_{0}^{R}\right\|^{2}+\left\|w_{y}^{R}\right\|^{2}+2\left\langle w_{0}^{R}, w_{y}^{R}\right\rangle \\
& -\int_{\mathbb{R}^{N}}(1+a(x)) \frac{f\left(w_{0}^{R}+w_{y}^{R}\right)}{w_{0}^{R}+w_{y}^{R}}\left(w_{0}^{R}+w_{y}^{R}\right)^{2} \mathrm{~d} x .
\end{aligned}
$$

Como $\frac{f(t)}{t}$ é crescente se $t>0$, podemos reescrever (1.91) como

$$
\begin{aligned}
J\left(2 z_{\frac{1}{2}, y}^{R}\right) \leq & \left\|w_{0}^{R}\right\|^{2}+\left\|w_{y}^{R}\right\|^{2}+2\left\langle w_{0}^{R}, w_{y}^{R}\right\rangle \\
& -\int_{\mathbb{R}^{N}}\left(1+a(x) \frac{f\left(w_{0}^{R}\right)}{w_{0}^{R}}\left(w_{0}^{R}\right)^{2} \mathrm{~d} x-\int_{\mathbb{R}^{N}}(1+a(x)) \frac{f\left(w_{y}^{R}\right)}{w_{y}^{R}}\left(w_{y}^{R}\right)^{2} \mathrm{~d} x\right. \\
& -\int_{\mathbb{R}^{N}}(1+a(x)) \frac{f\left(w_{0}^{R}+w_{y}^{R}\right)}{w_{0}^{R}+w_{y}^{R}}\left(2 w_{0}^{R} w_{y}^{R}\right) \mathrm{d} x \\
(i) \leq & \left\|w_{0}^{R}\right\|^{2}+\left\|w_{y}^{R}\right\|^{2}-\int_{\mathbb{R}^{N}} f\left(w_{0}^{R}\right) w_{0}^{R} \mathrm{~d} x-\int_{\mathbb{R}^{N}} f\left(w_{y}^{R}\right) w_{y}^{R} \mathrm{~d} x \\
& +2\left\langle w_{0}^{R}, w_{y}^{R}\right\rangle \\
(\text { ii }) \quad & -\int_{\mathbb{R}^{N}} a(x)\left[f\left(w_{0}^{R}\right) w_{0}^{R}+f\left(w_{y}^{R}\right) w_{y}^{R}\right] \mathrm{d} x .
\end{aligned}
$$

Vamos agora observar o que acontece em $(i),($ ii $),($ iii $)$. Em $(i)$, note que $w_{0}^{R}$ satisfaz

$$
-\Delta w_{0}^{R}+w_{0}^{R}=f\left(w_{0}^{R}\right) \text { em } \mathbb{R}^{N}
$$

visto que $w$ é solução do problema limite. Segue que $\left\|w_{0}^{R}\right\|^{2}-\int_{\mathbb{R}^{N}} f\left(w_{0}^{R}\right)\left(w_{0}^{R}\right) \mathrm{d} x=0$.

O mesmo resultado acontece para $w_{y}^{R}$. Portanto, o caso $(i)$ é igual a zero. Em $(i i)$, temos que $2\left\langle w_{0}^{R}, w_{y}^{R}\right\rangle=$ $o_{R}(1)$ como já provamos em (1.40). Em $(i i i)$, usando $\left(a_{2}\right),\left(f_{2}\right)$ e o Lema 1.9 afirmamos que

$$
\left|-\int_{\mathbb{R}^{N}} a(x)\left[f\left(w_{0}^{R}\right) w_{0}^{R}+f\left(w_{y}^{R}\right) w_{y}^{R}\right] \mathrm{d} x\right|=o_{R}(1) .
$$

De fato, note que

$$
\begin{aligned}
\left|-\int_{\mathbb{R}^{N}} a(x) f\left(w_{0}^{R}\right) w_{0}^{R} \mathrm{~d} x\right| & \leq C \int_{\mathbb{R}^{N}} e^{-k|x|}\left[\left|w_{0}^{R}\right|^{p_{1}+1}+\left|w_{0}^{R}\right|^{p_{2}+1}\right] \mathrm{d} x \\
& \leq C \int_{\mathbb{R}^{N}} e^{-k|x|}\left[e^{-\left(p_{1}+1\right)\left|x-R y_{0}\right|}+e^{-\left(p_{2}+1\right)\left|x-R y_{0}\right|}\right] \mathrm{d} x \\
& \leq C e^{-k\left|0-R y_{0}\right|}+C e^{-k\left|0-R y_{0}\right|} \\
& =C e^{-k R}=o_{R}(1)
\end{aligned}
$$

e similarmente para $\left|-\int_{\mathbb{R}^{N}} a(x) f\left(w_{y}^{R}\right) w_{y}^{R} \mathrm{~d} x\right|$. Assim, obtemos (1.92).

Combinandos os resultados em $(i),(i i),($ iii $)$ e (1.92) segue que para $\lambda=\frac{1}{2}$, uniformemente em $y \in$ 
$\partial B_{2}\left(y_{0}\right)$, se $R \rightarrow+\infty$,

$$
J\left(2 z_{\frac{1}{2}, y}^{R}\right)=J\left(w_{0}^{R}+w_{y}^{R}\right) \rightarrow 0 .
$$

Resta provar que

$$
T_{\frac{1}{2}, y}^{R} \rightarrow 2, \text { quando } R \rightarrow \infty .
$$

Argumentando por contradição, suponha que suponha que existam $\delta>0$ e subsequências $R_{n} \rightarrow \infty$ e $y_{n} \in \partial B_{2}\left(y_{0}\right)$ tais que

$$
T_{n}:=T_{\frac{1}{2}, y_{n}}^{R_{n}} \quad \text { satisfaz }\left|T_{n}-2\right| \geq \delta .
$$

Como $\left\{T_{n}\right\}$ é limitada pelo Lema 1.10, então existe uma constante $T$ tal que

$$
T_{n} \rightarrow T \text {, quando } n \rightarrow \infty \text {. }
$$

Por (1.5), se $t u \in \mathcal{N}$, então

$$
\|u\|^{2}=\int_{R^{N}}(1+a(x)) \frac{f(t u)}{t u} u^{2} \mathrm{~d} x .
$$

Por um lado, note que

$$
\begin{aligned}
\left\|z_{\frac{1}{2}, y_{n}}^{R_{n}}\right\|^{2} & =\left\|\frac{1}{2} w_{y_{0}}^{R_{n}}+\frac{1}{2} w_{y_{n}}^{R_{n}}\right\|^{2}=\frac{1}{4}\left\|w_{y_{0}}^{R_{n}}\right\|^{2}+\frac{1}{4}\left\|w_{y_{n}}^{R_{n}}\right\|^{2}+\frac{1}{2}\left\langle w_{y_{0}}^{R_{n}}, w_{y_{n}}^{R_{n}}\right\rangle \\
& =\frac{1}{4}\|w\|^{2}+\frac{1}{4}\|w\|^{2}+o_{R_{n}}(1) .
\end{aligned}
$$

Por outro lado, por $\left(a_{1}\right)$, mudança de variáveis e pelo Teorema da Convergência Dominada de Lebesgue obtemos

$$
\begin{aligned}
& \int_{\mathbb{R}^{N}}(1+a(x)) \frac{f\left(\frac{T_{n}}{2}\left(w_{y_{0}}^{R_{n}}+w_{y_{n}}^{R_{n}}\right)\right)}{\frac{T_{n}}{2}\left(w_{y_{0}}^{R_{n}}+w_{y_{n}}^{R_{n}}\right)}\left(\frac{1}{4}\left(w_{y_{0}}^{R_{n}}\right)^{2}+\frac{1}{4}\left(w_{y_{n}}^{R_{n}}\right)^{2}+\frac{1}{2} w_{y_{0}}^{R_{n}} w_{y_{n}}^{R_{n}}\right) \mathrm{d} x \\
& =\int_{\mathbb{R}^{N}}\left(1+a\left(x+R_{n} y_{0}\right)\right) \frac{f\left(\frac{T_{n}}{2}\left(w+w_{y_{n}+y_{0}}^{R_{n}}\right)\right)}{\frac{T_{n}}{2}\left(w+w_{y_{n}+y_{0}}^{R_{n}}\right)}\left(\frac{1}{4} w^{2}+\frac{1}{4}\left(w_{y_{n}+y_{0}}^{R_{n}}\right)^{2}+\frac{1}{2} w w_{y_{n}+y_{0}}^{R_{n}}\right) \mathrm{d} x \\
& \stackrel{n \rightarrow \infty}{\longrightarrow} \int_{\mathbb{R}^{N}} \frac{f\left(\frac{T}{2} w\right)}{\frac{T}{2} w}\left(\frac{2}{4} w^{2}\right) \mathrm{d} x
\end{aligned}
$$

De (1.94) e (1.95) resulta que

$$
\frac{2}{4}\|w\|^{2}=\int_{\mathbb{R}^{N}} \frac{f\left(\frac{T}{2} w\right)}{\frac{T}{2} w}\left(\frac{2}{4} w^{2}\right) \mathrm{d} x
$$

e desde que $w$ é solução do problema limite, segue que

$$
\int_{\mathbb{R}^{N}}\left(\frac{f(w)}{w}-\frac{f\left(\frac{T}{2} w\right)}{\frac{T}{2} w}\right) w^{2} \mathrm{~d} x=0 .
$$

Como $\frac{f(s)}{s}$ é crescente para $s>0$, obtemos $T=2$. Contradição com (1.93). Portanto, $T_{\frac{1}{2}, y}^{R} \rightarrow$ 2 , quando $R \rightarrow \infty$ e o lema está provado. 
Lema 1.18. Existem números $R_{2}>0$ e $\alpha>0$ tal que

$$
I\left(T_{\lambda, y}^{R} z_{\lambda, y}^{R}\right) \leq 2 m_{\infty}-\alpha
$$

para cada $R>R_{2}$ e para todo $y \in \partial B_{2}\left(y_{0}\right)$ e $\lambda \in[0,1]$.

Demonstração: Primeiramente escrevemos a combinação linear

$$
\lambda T_{\lambda, y}^{R} w\left(x-R y_{0}\right)+(1-\lambda) T_{\lambda, y}^{R} w(x-R y):=s w_{0}^{R}+t w_{y}^{R} .
$$

Já sabemos pelo Lema 1.10 que $s$ e $t$ pertencem ao intervalo $\left(0, T_{0}\right)$, ou seja, já sabemos que são limitados. Tomando $u=s w_{0}^{R}+t w_{y}^{R}$ em $I$ definido em (1.2) obtemos

$$
\begin{aligned}
& I\left(s w_{0}^{R}+t w_{y}^{R}\right)= \frac{1}{2} \int_{R^{N}}\left(s^{2}\left|\nabla w_{0}^{R}\right|^{2}+t^{2}\left|\nabla w_{y}^{R}\right|^{2}+2 s t \nabla w_{0}^{R} \nabla w_{y}^{R}\right) \mathrm{d} x \\
&+\frac{1}{2} \int_{R^{N}}\left(s^{2}\left|w_{0}^{R}\right|^{2}+t^{2}\left|w_{y}^{R}\right|^{2}+2 s t w_{0}^{R} w_{y}^{R}\right) \mathrm{d} x \\
&-\int_{R^{N}}(1+a(x)) F\left(s w_{0}^{R}+t w_{y}^{R}\right) \mathrm{d} x \\
&(A) \quad \frac{s^{2}}{2} \int_{R^{N}}\left(\left|\nabla w_{0}^{R}\right|^{2}+\left(w_{0}^{R}\right)^{2}\right) \mathrm{d} x+\frac{t^{2}}{2} \int_{R^{N}}\left(\left|\nabla w_{y}^{R}\right|^{2}+\left(w_{y}^{R}\right)^{2}\right) \mathrm{d} x \\
&(B) \quad-\int_{R^{N}} F\left(s w_{0}^{R}\right) \mathrm{d} x-\int_{R^{N}} F\left(t w_{y}^{R}\right) \mathrm{d} x \\
&-\int_{R^{N}}\left[F\left(s w_{0}^{R}+t w_{y}^{R}\right)-F\left(s w_{0}^{R}\right)-F\left(t w_{y}^{R}\right)\right] d x \\
&(C) \quad s t \int_{R^{N}}\left(\nabla w_{0}^{R} \nabla w_{y}^{R}+w_{0}^{R} w_{y}^{R}\right) \mathrm{d} x-\int_{R^{N}}\left(f\left(s w_{0}^{R}\right) t w_{y}^{R}+f\left(t w_{y}^{R}\right) s w_{0}^{R}\right) \mathrm{d} x \\
&(D) \quad \\
&+\int_{R^{N}}\left(f\left(s w_{0}^{R}\right) t w_{y}^{R}+f\left(t w_{y}^{R}\right) s w_{0}^{R}\right) \mathrm{d} x \\
&(E) \quad \int_{R^{N}}(1+a(x)) F\left(s w_{0}^{R}+t w_{y}^{R}\right) \mathrm{d} x+\int_{R^{N}} F\left(s w_{0}^{R}+t w_{y}^{R}\right) \mathrm{d} x . \\
&(F) \quad
\end{aligned}
$$

Vamos estimar cada linha em (1.97). Nas linhas $(A)$ e $(B)$ tem-se

$$
\frac{s^{2}}{2} \int_{R^{N}}\left(\left|\nabla w_{0}^{R}\right|^{2}+\left(w_{0}^{R}\right)^{2}\right) \mathrm{d} x-\int_{R^{N}} F\left(s w_{0}^{R}\right) \mathrm{d} x=I_{\infty}\left(s w_{0}^{R}\right) \leq m_{\infty}
$$

e

$$
\frac{t^{2}}{2} \int_{R^{N}}\left(\left|\nabla w_{0}^{R}\right|^{2}+\left(w_{0}^{R}\right)^{2}\right) \mathrm{d} x-\int_{R^{N}} F\left(t w_{0}^{R}\right) \mathrm{d} x=I_{\infty}\left(t w_{0}^{R}\right) \leq m_{\infty} .
$$

Na linha $(C)$, utilizamos o Lema 1.14 para obter

$$
\begin{aligned}
& \int_{R^{N}}\left[F\left(s w_{0}^{R}+t w_{y}^{R}\right)-F\left(s w_{0}^{R}\right)-F\left(t w_{y}^{R}\right)\right] \mathrm{d} x \\
\geq & t \int_{R^{N}} f\left(s w_{0}^{R}\right) w_{y}^{R} \mathrm{~d} x+s \int_{R^{N}} f\left(t w_{y}^{R}\right) w_{0}^{R} \mathrm{~d} x-C_{\rho}(s t)^{1+\frac{\mu}{2}} \int_{R^{N}}\left(w_{0}^{R}\right)^{1+\frac{\mu}{2}}\left(w_{y}^{R}\right)^{1+\frac{\mu}{2}} \mathrm{~d} x(1.100)
\end{aligned}
$$


Tomando $1<\bar{\mu}<1+\frac{\mu}{2}$ e o Lema 1.9 implica que

$$
\begin{aligned}
\int_{R^{N}}\left(w_{0}^{R}\right)^{1+\frac{\mu}{2}}\left(w_{y}^{R}\right)^{1+\frac{\mu}{2}} \mathrm{~d} x & \leq C \int_{R^{N}} e^{-\left(1+\frac{\mu}{2}\right)\left|x-R y_{0}\right|} e^{-\left(1+\frac{\mu}{2}\right)|x-R y|} \mathrm{d} x \\
& \leq C e^{-\bar{\mu}\left|R y_{0}-R y\right|}=C e^{-2 \bar{\mu} R}=o\left(\varepsilon_{R}\right) .
\end{aligned}
$$

Portanto, segue de (1.100) e (1.101) que

$$
\begin{aligned}
& -\int_{R^{N}}\left[F\left(s w_{0}^{R}+t w_{y}^{R}\right)-F\left(s w_{0}^{R}\right)-F\left(t w_{y}^{R}\right)\right] \mathrm{d} x \\
\leq & -t \int_{R^{N}} f\left(s w_{0}^{R}\right) w_{y}^{R} \mathrm{~d} x-s \int_{R^{N}} f\left(t w_{y}^{R}\right) w_{0}^{R} \mathrm{~d} x+o\left(\varepsilon_{R}\right) .
\end{aligned}
$$

Combinando (1.102) e (E) obtemos

$$
(C)+(E) \leq o\left(\varepsilon_{R}\right) .
$$

Para a linha $(D)$, usamos o Corolário 1.1 e o Lema 1.16 para obter

$$
\begin{aligned}
& s t \int_{R^{N}}\left(\nabla w_{0}^{R} \nabla w_{y}^{R}+w_{0}^{R} w_{y}^{R}\right) \mathrm{d} x-\int_{R^{N}}\left(f\left(s w_{0}^{R}\right) t w_{y}^{R}+f\left(t w_{y}^{R}\right) s w_{0}^{R}\right) \mathrm{d} x \\
= & \frac{s t}{2} \int_{R^{N}}\left(\nabla w_{0}^{R} \nabla w_{y}^{R}+w_{0}^{R} w_{y}^{R}\right) \mathrm{d} x-\frac{1}{2} \int_{R^{N}} f\left(s w_{0}^{R}\right) t w_{y}^{R} \mathrm{~d} x \\
& +\frac{s t}{2} \int_{R^{N}}\left(\nabla w_{y}^{R} \nabla w_{0}^{R}+w_{y}^{R} w_{0}^{R}\right) \mathrm{d} x-\frac{1}{2} \int_{R^{N}} f\left(t w_{y}^{R}\right) s w_{0}^{R} \mathrm{~d} x \\
& \quad-\frac{1}{2} \int_{R^{N}}\left(f\left(s w_{0}^{R}\right) t w_{y}^{R}+f\left(t w_{y}^{R}\right) s w_{0}^{R}\right) \mathrm{d} x \\
= & \frac{t}{2} \int_{R^{N}}\left(s f\left(w_{0}^{R}\right)-f\left(s w_{0}^{R}\right)\right) w_{y}^{R} \mathrm{~d} x+\frac{s}{2} \int_{R^{N}}\left(t f\left(w_{y}^{R}\right)-f\left(t w_{y}^{R}\right)\right) w_{0}^{R} \\
& \quad-\frac{1}{2} \int_{R^{N}}\left(f\left(s w_{0}^{R}\right) t w_{y}^{R}+f\left(t w_{y}^{R}\right) s w_{0}^{R}\right) \mathrm{d} x \\
\leq & C(|s-1|+|t-1|) O\left(\varepsilon_{R}\right)-C_{0} \varepsilon_{R} .
\end{aligned}
$$

Resta-nos estimar a linha $(F)$, isto é,

$$
-\int_{R^{N}}(1+a(x)) F\left(s w_{0}^{R}+t w_{y}^{R}\right) \mathrm{d} x+\int_{R^{N}} F\left(s w_{0}^{R}+t w_{y}^{R}\right) \mathrm{d} x=-\int_{R^{N}} a(x) F\left(s w_{0}^{R}+t w_{y}^{R}\right) \mathrm{d} x .
$$

Pela hipótese $\left(a_{2}\right)$ e o Lema 1.9 segue que

$$
\begin{aligned}
& \left|-\int_{R^{N}} a(x) F\left(s w_{0}^{R}+t w_{y}^{R}\right) \mathrm{d} x\right| \\
\leq & C \int_{\mathbb{R}^{N}} e^{-k|x|}\left(\left|w_{0}^{R}\right|^{p_{1}+1}+\left|w_{0}^{R}\right|^{p_{2}+1}+\left|w_{y}^{R}\right|^{p_{1}+1}+\left|w_{y}^{R}\right|^{p_{2}+1}\right) \mathrm{d} x \\
\leq & C e^{-k\left|0-R y_{0}\right|}+C e^{-k|0-R y|} \leq C e^{-k R}=o\left(\varepsilon_{R}\right) .
\end{aligned}
$$

Resulta do Lema 1.17 que se $\lambda=\frac{1}{2}$, então $s, t \rightarrow 1$, quando $R \rightarrow+\infty$. Tomando $R_{2}$ suficientemente grande e $\sigma=\sigma(R) \in\left(0, \frac{1}{2}\right)$ segue por (1.98), (1.99), (1.100), (1.101), (1.102), (1.103), (1.104), (1.105) 
que

$$
I\left(s w_{0}^{R}+t w_{y}^{R}\right)=I\left(\lambda T_{\lambda, y}^{R} w\left(x-R y_{0}\right)+(1-\lambda)\right) T_{\lambda, y}^{R} w(x-R y) \leq 2 m_{\infty}-\frac{C}{3} \varepsilon_{R}+o\left(\varepsilon_{R}\right) .
$$

Por continuidade, para todo $\lambda \in\left[\frac{1}{2}-\sigma, \frac{1}{2}+\sigma\right], y \in \partial B_{2}\left(y_{0}\right), R>R_{2}$, vale que

$$
I\left(s w_{0}^{R}+t w_{y}^{R}\right)=I\left(\lambda T_{\lambda, y}^{R} w\left(x-R y_{0}\right)+(1-\lambda)\right) T_{\lambda, y}^{R} w(x-R y) \leq 2 m_{\infty}-\frac{C}{3} \varepsilon_{R}+o\left(\varepsilon_{R}\right) .
$$

Resta analisar o caso $\lambda \in\left[0, \frac{1}{2}-\sigma\right] \cup\left[\frac{1}{2}+\sigma, 1\right]$. Para isto, sem perda de generalidade, fixemos $\lambda$ tal que $0 \leq \lambda<\frac{1}{2}-\sigma$. Então $1 \geq 1-\lambda>\frac{1}{2}+\sigma$. Note que, se $T_{\lambda, y}^{R} \leq 2$, então $s=T_{\lambda, y}^{R} \lambda \in[0,1-2 \sigma]$ e $t=T_{\lambda, y}^{R}(1-\lambda) \in[1+2 \sigma, 2]$, ou seja, temos $s<1$ e $t>1$. Por outro lado, se $T_{\lambda, y}^{R} \geq 2$, então $s=T_{\lambda, y}^{R} \lambda \in[1-2 \sigma,+\infty]$ e $t=T_{\lambda, y}^{R}(1-\lambda) \in[1+2 \sigma,+\infty]$. Ademais, sabemos que sendo $w$ solução positiva ground state do problema limite, utilizando o Lema 1.1 segue que $I_{\infty}\left(t w_{0}^{R}\right)<m_{\infty}-\eta$ para algum $\eta \in\left(0, m_{\infty}\right), t \in\left[0,1-\sigma_{0}\right] \cup\left[1+\sigma_{0}, \infty\right)$, para algum $\sigma_{0}$. Juntando isto e as estimativas anteriores de (1.98) a (1.105) mostramos que

$$
I\left(s w_{0}^{R}+t w_{y}^{R}\right)=I\left(\lambda T_{\lambda, y}^{R} w\left(x-R y_{0}\right)+(1-\lambda)\right) T_{\lambda, y}^{R} w(x-R y) \leq m_{\infty}-\eta+m_{\infty}-\eta+O\left(\varepsilon_{R}\right),
$$

$\forall \lambda \in\left[0, \frac{1}{2}-\sigma\right] \cup\left[\frac{1}{2}+\sigma, 1\right]$.

Portanto, concluímos o lema juntando os resultados em (1.106), (1.107) e (1.108) para todo $\lambda \in[0,1]$, $y \in \partial B_{2}\left(y_{0}\right), R>R_{2}$.

Lema 1.19. Dado número real $\eta>0$, existe um número real $R_{3}>0$ tal que

$$
I\left(T_{0, y}^{R} z_{0, y}^{R}\right)<m_{\infty}+\eta
$$

para todo $y \in \partial B_{2}\left(y_{0}\right), R>R_{3}$. Em particular, $m \leq m_{\infty}$.

Demonstração: Inicialmente iremos mostrar que

$$
\int_{\mathbb{R}^{N}}|a(x)|\left|F\left(T_{0, y}^{R} w_{y}^{R}\right)\right| \mathrm{d} x=o_{R}(1) .
$$


De fato, por $\left(a_{2}\right),\left(f_{2}\right),(1.24)$ e o Lema 1.9 existe uma constante $C>0$ tal que

$$
\begin{aligned}
\int_{\mathbb{R}^{N}}|a(x)|\left|F\left(T_{0, y}^{R} w_{y}^{R}\right)\right| \mathrm{d} x \leq & C \int_{\mathbb{R}^{N}}|a(x)|\left(\left|T_{0, y}^{R} w_{y}^{R}\right|^{p_{1}+1}+\left|T_{0, y}^{R} w_{y}^{R}\right|^{p_{2}+1}\right) \mathrm{d} x \\
\leq & C \int_{\mathbb{R}^{N}} e^{-k|x|}\left(\left|T_{0, y}^{R} w_{y}^{R}\right|^{p_{1}+1}+\left|T_{0, y}^{R} w_{y}^{R}\right|^{p_{2}+1}\right) \mathrm{d} x \\
= & C \int_{\mathbb{R}^{N}} e^{-k|x|}\left(\left(T_{0, y}^{R}\right)^{p_{1}+1}\left|w_{y}^{R}\right|^{p_{1}+1}+\left(T_{0, y}^{R}\right)^{p_{2}+1}\left|w_{y}^{R}\right|^{p_{2}+1}\right) \mathrm{d} x \\
\leq & C\left(T_{0, y}^{R}\right)^{p_{1}+1} \int_{\mathbb{R}^{N}} e^{-k|x|} e^{-\left(p_{1}+1\right)|x-R y|} \mathrm{d} x \\
& +C\left(T_{0, y}^{R}\right)^{p_{2}+1} \int_{\mathbb{R}^{N}} e^{-k|x|} e^{-\left(p_{2}+1\right)|x-R y|} \mathrm{d} x \\
\leq & C\left(T_{0, y}^{R}\right)^{p_{1}+1} e^{-k|0-R y|}+C\left(T_{0, y}^{R}\right)^{p_{2}+1} e^{-k|0-R y|} \\
\leq & C\left(T_{0, y}^{R}\right)^{p_{1}+1} e^{-k R}+C\left(T_{0, y}^{R}\right)^{p_{2}+1} e^{-k R}=o\left(\varepsilon_{R}\right)
\end{aligned}
$$

com $2<k<p_{i}+1 ; i \in\{1,2\}$ e $T_{0, y}^{R}$ é limitado.

Considerando $\lambda=0$ na equação (1.96) temos

$$
T_{0, y}^{R} z_{0, y}^{R}=T_{0, y}^{R} w(x-R y):=T_{0, y}^{R} w_{y}^{R} .
$$

Usando o Lema 1.1(c) e tomando $u=T_{0, y}^{R} w_{y}^{R}$ em (1.2) segue que

$$
\begin{aligned}
I\left(T_{0, y}^{R} w_{y}^{R}\right) & =I_{\infty}\left(T_{0, y}^{R} w_{y}^{R}\right)-\int_{\mathbb{R}^{N}} a(x) F\left(T_{0, y}^{R} w_{y}^{R}\right) \mathrm{d} x \\
& \leq \max _{t>0} I_{\infty}\left(t w_{y}^{R}\right)+\int_{\mathbb{R}^{N}}|a(x)|\left|F\left(T_{0, y}^{R} w_{y}^{R}\right)\right| \mathrm{d} x \\
& =I_{\infty}\left(w_{y}^{R}\right)+\int_{\mathbb{R}^{N}}|a(x)|\left|F\left(T_{0, y}^{R} w_{y}^{R}\right)\right| \mathrm{d} x \\
& =m_{\infty}+\int_{\mathbb{R}^{N}}|a(x)|\left|F\left(T_{0, y}^{R} w_{y}^{R}\right)\right| \mathrm{d} x .
\end{aligned}
$$

Combinando (1.109) e (1.110), o lema está provado.

\subsection{Demonstração do resultado principal}

Para a demonstrar o teorema principal deste capítulo utilizaremos ferramentas do tipo "min-max". Precisaremos introduzir a função baricentro $\beta: H^{1}\left(\mathbb{R}^{N}\right) \backslash\{0\} \rightarrow \mathbb{R}^{N}$. Inicialmente definiremos as funções $\Psi_{u}, v: \mathbb{R}^{N} \rightarrow \mathbb{R}$ por

$$
\Psi_{u}:=\frac{1}{\mu\left(B_{1}(0)\right)} \int_{B_{1}(x)}|u(y)| \mathrm{d} y \quad \text { e } \quad v(x):=\left(\Psi_{u}-\frac{1}{2} \max _{x \in \mathbb{R}^{N}} \Psi_{u}(x)\right)^{+},
$$

onde $\mu\left(B_{1}(0)\right)$ é a medida de Lebesgue da bola unitária. A função baricentro de $u$ é a aplicação definida por

$$
\beta(u):=\frac{1}{|v|_{1}} \int_{\mathbb{R}^{N}} x v(x) \mathrm{d} x .
$$


A função baricentro possui as seguintes propriedades:

i) $\beta$ é contínua em $H^{1}\left(\mathbb{R}^{N}\right) \backslash\{0\}$;

ii) Se $u \in H^{1}\left(\mathbb{R}^{N}\right) \backslash\{0\}$ é radialmente simétrica, então $\beta(u)=0$;

iii) Para todo $t \in \mathbb{R} \backslash\{0\}$ e todo $u \in H^{1}\left(\mathbb{R}^{N}\right) \backslash\{0\}$ tem-se $\beta(u)=\beta(t u)$;

iv) Dado $z \in \mathbb{R}^{N}$ tem-se $\beta(u(x-z))=\beta(u)+z$.

Definida a função baricentro, podemos demonstrar o seguinte resultado:

Lema 1.20. Suponha que $m$ não seja atingido. Então $m=m_{\infty}$ e existe um $\eta>0$ tal que

$$
\beta(u) \neq 0, \text { para toda } u \in \mathcal{N} \cap I^{m_{\infty}+\eta}
$$

onde denotamos

$$
I^{m_{\infty}+\eta}:=\left\{u \in H^{1}\left(\mathbb{R}^{N}\right) ; \quad I(u) \leq m_{\infty}+\eta\right\} .
$$

Demonstração: Suponha que $m$ não é atingido. Pelo Lema 1.8, $m \geq m_{\infty}$ e do Lema 1.19, a desigualdade invertida. Logo temos a igualdade $m=m_{\infty}$.

Argumentando por contradição, suponha que para cada $n \in \mathbb{N}$ exista $\left\{v_{n}\right\} \subset \mathcal{N} \cap I^{m_{\infty}+\alpha}$, isto é, $\left\{v_{n}\right\} \subset \mathcal{N}$ satisfazendo $I\left(v_{n}\right)<m_{\infty}+o_{n}(1)$ e, ainda, $\beta\left(v_{n}\right)=0$. Segue disto que $\left\{v_{n}\right\} \subset \mathcal{N}$ é uma sequência minimizante para $I$. O Princípio Variacional de Ekeland, por instante vide [46], nos fornece uma sequência $\left\{u_{n}\right\}$ para $I$ restrito $\mathcal{N}$ no nível $m_{\infty}$ onde tal sequência é (PS) e satisfaz

$$
\left\|v_{n}-u_{n}\right\| \rightarrow 0 \text {, quando } n \rightarrow \infty \text {. }
$$

Já vimos pelo Lema 1.2 e 1.5 que toda sequência (PS) em $\mathcal{N}$ é limitada e como $m$ não é atingido, podemos usar o Lema 1.7 para garantir a existência de uma sequência

$$
\left\{z_{n}\right\} \subset \mathbb{R}^{N} ;\left\|z_{n}\right\| \rightarrow \infty \text { e }\left\|u_{n}-w\left(\cdot-z_{n}\right)\right\| \rightarrow 0, \text { quando } n \rightarrow \infty
$$

onde $w$ é a solução positiva, radial de energia mínima do problema limite.

Por translação temos

$$
u_{n}\left(x+z_{n}\right)=w(x)+o_{n}(1) .
$$

Usando as propriedades da função baricentro e que $\beta\left(v_{n}\right)=0$ temos

$$
\beta\left(v_{n}\left(x+z_{n}\right)\right)=\beta\left(v_{n}\right)-z_{n}=-z_{n}
$$

e, ainda, pela continuidade da função baricentro $\beta$ na norma em $H^{1}\left(\mathbb{R}^{N}\right)$

$$
\beta\left(v_{n}\left(x+z_{n}\right)\right) \rightarrow \beta(w(x))=0,
$$

que configura uma contradição, pois $\left\|z_{n}\right\| \rightarrow \infty$. 
Por fim iremos demonstrar o resultado principal onde utilizaremos os resultados que obtivemos anteriormente.

\section{Prova do Teorema 0.1:}

Inicialmente, suponha que $m$ seja atingido para algum $u \in \mathcal{N}$. Consequentemente, pelos Lemas 1.1(b) e 1.3 temos que $u$ é uma solução não trivial para o problema $(P)$. Suponha então que $m$ não seja atingido. Segue do Lema 1.20 que $m=m_{\infty}, \beta(u) \neq 0$, para toda $u \in \mathcal{N} \cap I^{m_{\infty}+\eta}$ e podemos fixar $\eta$ entre $\left(0, \frac{m_{\infty}}{8}\right)$ e, ainda pelo Lema 1.19, para todo $R>R_{3}$

$$
I\left(T_{0, y}^{R} z_{0, y}^{R}\right) \leq m_{\infty}+\eta, \quad \forall y \in \partial B_{2}\left(y_{0}\right)
$$

Pelo Lema 1.18 podemos escolher $\alpha$ entre $\left(0, \frac{m_{\infty}}{8}\right)$ e para todo $R>R_{2}$

$$
I\left(T_{\lambda, y}^{R} z_{\lambda, y}^{R}\right) \leq 2 m_{\infty}-\alpha, \quad \forall y \in \partial B_{2}\left(y_{0}\right), \quad \lambda \in[0,1] .
$$

Considere $R>\max \left\{R_{2}, R_{3}\right\}$ e defina a seguinte aplicação

$$
\begin{array}{cl}
\mathcal{H}: B_{2}(0) & \longrightarrow \mathcal{N} \cap I^{2 m_{\infty}-\eta} \\
\lambda y_{0}+(1-\lambda) y & \longrightarrow T_{\lambda, y}^{R} z_{\lambda, y}^{R}, \forall y \in \partial B_{2}\left(y_{0}\right), \quad \lambda \in[0,1] .
\end{array}
$$

O objetivo agora é mostrar que o funcional $I$ tem um valor crítico no intervalo $\left(m_{\infty}, 2 m_{\infty}\right)$. Argumentando por contradição, suponha que tal valor crítico não exista . Como $m$ não é atingido, podemos utilizar o Lema 1.8 para garantir que o funcional $I$ satisfaz a condição de Palais - Smale no intervalo $\left(m_{\infty}, 2 m_{\infty}\right)$. Logo, existe um $\varepsilon>0$ tal que

$$
\left\|\left(\left.I\right|_{\mathcal{N}}\right)^{\prime}(u)\right\| \geq \varepsilon \text { para todo } u \in \mathcal{N} \cap I^{-1}\left[m_{\infty}+\eta, 2 m_{\infty}-\alpha\right] .
$$

Isso implica que, pelo Lema 5.15 em [46], existe uma aplicação (deformação) contínua

$$
\mathcal{D}: \mathcal{N} \cap I^{2 m_{\infty}-\alpha} \rightarrow \mathcal{N} \cap I^{m_{\infty}+\eta}
$$

tal que $\mathcal{D}=i d$ (aplicação identidade) para todo $u \in \mathcal{N} \cap I^{m_{\infty}+\eta}$.

Por (1.111), (1.112), (1.113) e (1.114) podemos definir a seguinte aplicação contínua

$$
\begin{aligned}
& \Gamma: B_{2}(0) \quad \rightarrow \quad \partial B_{2}(0) \\
& x \quad \rightarrow \quad A_{2}\left(2 \frac{\beta o \mathcal{D} o \mathcal{H} o A_{1}(x)}{\mid \beta o \mathcal{D} \text { Ho } A_{1}(x) \mid}\right),
\end{aligned}
$$

onde $\beta$ é a aplicação baricentro e as aplicações contínuas $A_{1}$ e $A_{2}$ são definidas como segue

$$
\begin{aligned}
& A_{1}: B_{2}(0) \rightarrow B_{2}\left(y_{0}\right) \\
& x \quad \rightarrow \quad x+y_{0}
\end{aligned}
$$

e

$$
\begin{array}{cl}
A_{2}: \partial B_{2}(0) & \rightarrow \partial B_{2}(0) \\
\frac{2 y}{|y|} & \rightarrow y-y_{0}
\end{array}
$$


onde $y \in \partial B_{2}\left(y_{0}\right)$. Além disso, se $\lambda=0$ segue que

$$
\mathcal{H}(y)=T_{0, y}^{R} Z_{0, y}^{R}
$$

Por outro lado, usando as propriedades da função baricentro note que

$$
\beta\left(T_{0, y}^{R} z_{0, y}^{R}\right)=\beta\left(T_{0, y}^{R} \omega_{y}^{R}\right)=\beta\left(\omega_{y}^{R}\right)=\beta(\omega(x-R y))=\beta(\omega(x))+R y=R y .
$$

Como $\mathcal{D}=i d$ para todo $u \in \mathcal{N} \cap I^{m_{\infty}+\eta}$ tem-se por $(1.115),(1.116),(1.117),(1.118)$ e (1.119) que

$$
\Gamma\left(y-y_{0}\right)=A_{2}\left(2 \frac{\beta o \mathcal{D} o \mathcal{H} o A_{1}\left(y-y_{0}\right)}{\left|\beta o \mathcal{D} o \mathcal{H} o A_{1}\left(y-y_{0}\right)\right|}\right)=A_{2}\left(2 \frac{\beta\left(T_{0, y}^{R} z_{0, y}^{R}\right)}{\left|\beta\left(T_{0, y}^{R} z_{0, y}^{R}\right)\right|}\right)=A_{2}\left(2 \frac{R y}{|R y|}\right)=y-y_{0}
$$

Temos uma contradição visto que tal aplicação contínua $\Gamma$ não pode existir pelo Teorema do Ponto Fixo de Brouwer, veja Teorema 3.5 em [7]. Portanto, a contradição veio de supor que $I$ não tem um valor crítico no intervalo $\left(m_{\infty}, 2 m_{\infty}\right)$.

Concluimos a demonstração do Teorema observando a positividade da solução, pois a solução $u$ não muda de sinal pelo Lema 1.4. Ademais, como $f$ é uma função ímpar, $-u$ também é solução do problema $(P)$. Assim, o resultado segue e o teorema está provado. 


\section{Capítulo}

2

\section{Sistema fortemente acoplado}

Neste capítulo vamos investigar a existência de solução positiva para o seguinte sistema

$$
(\mathbb{S}) \begin{cases}-\Delta u+u=(1+a(x)) \frac{u\left(u^{2}+v^{2}\right)}{1+s\left(u^{2}+v^{2}\right)}+\lambda v, & \mathbb{R}^{N}, \\ -\Delta v+v=(1+a(x)) \frac{v\left(u^{2}+v^{2}\right)}{1+s\left(u^{2}+v^{2}\right)}+\lambda u, & \mathbb{R}^{N}, \\ u, v \in H^{1}\left(\mathbb{R}^{N}\right), & \end{cases}
$$

com $N \geq 3,0<\lambda<1$ e $s$ é um parâmetro real satisfazendo $0<s<\frac{1}{1+\lambda}$.

Vamos assumir as seguintes hipóteses sobre a função peso:

$\left(a_{1}\right) a \in C\left(\mathbb{R}^{N}\right) ; \inf _{x \in \mathbb{R}^{N}}(1+a(x))=\varsigma>0$ e $\lim _{|x| \rightarrow+\infty} a(x)=0 ;$

$\left(a_{2}\right)|a(x)| \leq C e^{-k|x|}$ onde $k \in(2 \sqrt{1-\lambda}, 4 \sqrt{1-\lambda})$ para todo $x \in \mathbb{R}^{N}$ e $C$ uma constante positiva.

Considere

$$
f(u, v):=\frac{u\left(u^{2}+v^{2}\right)}{1+s\left(u^{2}+v^{2}\right)}, \quad g(u, v):=\frac{v\left(u^{2}+v^{2}\right)}{1+s\left(u^{2}+v^{2}\right)}
$$

e

$$
F(u, v):=\frac{u^{2}+v^{2}}{2 s}-\frac{1}{2 s^{2}} \ln \left(1+s\left(u^{2}+v^{2}\right)\right) \text { onde } \frac{\partial F}{\partial u}=f \quad \text { e } \quad \frac{\partial F}{\partial v}=g .
$$

A seguir serão feitas algumas afirmações com suas respectivas provas referentes a algumas particularidades do sistema.

Afirmação 2.1. A seguinte afirmação é verificada.

$$
\left(F_{1}\right) \frac{\nabla F(t u, t v)(u, v)}{t} \text { é crescente para } t>0 \text { onde } u, v \neq 0 \text {. }
$$

Demonstração: Considere a função

$$
G(t)=\frac{\nabla F(t u, t v)(u, v)}{t}=\frac{1}{t} \frac{(t u)^{2}+(t v)^{2}}{1+s\left((t u)^{2}+(t v)^{2}\right)}\left(t u^{2}+t v^{2}\right)=\frac{t^{2}\left(u^{2}+v^{2}\right)^{2}}{1+s t^{2}\left(u^{2}+v^{2}\right)} .
$$


Derivando $G(t)$ em $t$ obtemos

$$
\begin{aligned}
G^{\prime}(t) & =\frac{2 t\left(u^{2}+v^{2}\right)^{2}+2 s t^{3}\left(u^{2}+v^{2}\right)^{3}-2 s t^{3}\left(u^{2}+v^{2}\right)^{3}}{\left(1+s t^{2}\left(u^{2}+v^{2}\right)\right)^{2}} \\
& =\frac{2 t\left(u^{2}+v^{2}\right)^{2}}{\left(1+s t^{2}\left(u^{2}+v^{2}\right)\right)^{2}}>0 .
\end{aligned}
$$

Portanto, segue que $\frac{\nabla F(t u, t v)(u, v)}{t}$ é crescente em $t>0$.

Afirmação 2.2. A seguinte afirmação é verificada.

$\left(F_{2}\right)$ F satisfaz a condição de não quadraticidade :

(a) $\lim _{\|(u, v)\| \rightarrow \infty} \frac{1}{2} \nabla F(u, v)(u, v)-F(u, v)=+\infty$;

(b) $\frac{1}{2} \nabla F(u(x), v(x))(u(x), v(x))-F(u(x), v(x)) \geq 0$ para todo $x \in \mathbb{R}^{N}$.

Demonstração: Primeiramente mostraremos o item (b). Considere

$$
h(t):=\frac{t^{2}\left(u^{2}+v^{2}\right)^{2}}{2\left(1+s\left(u^{2}+v^{2}\right)\right)}-\frac{t^{2}\left(u^{2}+v^{2}\right)}{2 s}+\frac{1}{2 s^{2}} \ln \left(1+t^{2} s\left(u^{2}+v^{2}\right)\right),
$$

para todo $t \geq 0$. Derivando $h$ em $t$,

$$
h^{\prime}(t)=\frac{t\left(u^{2}+v^{2}\right)^{2}}{1+s\left(u^{2}+v^{2}\right)}-\frac{t^{3}\left(u^{2}+v^{2}\right)^{2}}{1+s t^{2}\left(u^{2}+v^{2}\right)},
$$

o que implica

$$
\begin{aligned}
& h^{\prime}(t)<0, \text { se } t>1 ; \\
& h^{\prime}(t)>0, \text { se } t<1 ; \\
& h^{\prime}(t)=0, \text { se } t=1 .
\end{aligned}
$$

Assim, $h(t) \leq h(1)$ para todo $t>0$. Ademais,

$$
\begin{aligned}
\frac{1}{2} \nabla F(u(x), v(x))(u(x), v(x))-F(u(x), v(x)) & =\frac{1}{2}\left(\frac{\left(u^{2}+v^{2}\right) u}{1+s\left(u^{2}+v^{2}\right)}, \frac{\left(u^{2}+v^{2}\right) v}{1+s\left(u^{2}+v^{2}\right)}\right)(u, v)-F(u, v) \\
& =\frac{1}{2}\left[\frac{\left(u^{2}+v^{2}\right) u^{2}}{1+s\left(u^{2}+v^{2}\right)}+\frac{\left(u^{2}+v^{2}\right) v^{2}}{1+s\left(u^{2}+v^{2}\right)}\right]-F(u, v) \\
& =\frac{\left(u^{2}+v^{2}\right)^{2}}{2\left(1+s\left(u^{2}+v^{2}\right)\right)}-\frac{u^{2}+v^{2}}{2 s}+\frac{1}{2 s^{2}} \ln \left(1+s\left(u^{2}+v^{2}\right)\right) \\
& =h(1)>h(0)=0, \quad \forall(u, v) \neq(0,0) .
\end{aligned}
$$

Portanto, este item fica verificado.

Verificaremos agora o item $(a)$. Para isso, considere a mudança de coordenadas $u^{2}+v^{2}=z^{2}$ e obtemos para todo $z^{2}>0$ que

$$
\frac{z^{4}}{2\left(1+s z^{2}\right)}-\frac{z^{2}}{2 s} \geq-\frac{1}{2 s^{2}}
$$


e fazendo $z \rightarrow+\infty$ temos

$$
\frac{z^{4}}{2\left(1+s z^{2}\right)}-\frac{z^{2}}{2 s}+\frac{1}{2 s^{2}} \ln \left(1+s z^{2}\right) \geq-\frac{1}{2 s^{2}}+\frac{1}{2 s^{2}} \ln \left(1+s z^{2}\right) \rightarrow+\infty .
$$

Portanto,

$$
\lim _{z \rightarrow+\infty}\left[\frac{z^{4}}{2\left(1+s z^{2}\right)}-\frac{z^{2}}{2 s}+\frac{1}{2 s^{2}} \ln \left(1+s z^{2}\right)\right]=+\infty .
$$

Assim, concluímos o item $(a)$.

Afirmação 2.3. Comportamento de $\nabla F$ na origem e no infinito.

$\left(F_{3}\right) \nabla F(u, v) \cdot(u, v)=o\left(u^{2}+v^{2}\right) \quad e \quad \lim _{\|(u, v)\| \rightarrow \infty} \frac{\nabla F(u, v) \cdot(u, v)}{u^{2}+v^{2}}=\frac{1}{s}$.

Demonstração: A verificação segue diretamente da não linearidade.

\subsection{Preliminares}

Iremos utilizar uma constante geral $C>0$ para simplificar a notação e assim $C$ não será sempre a mesma constante no que segue.

Consideramos o espaço de Hilbert $H:=H^{1}\left(\mathbb{R}^{N}\right) \times H^{1}\left(\mathbb{R}^{N}\right)$ com norma dada por

$$
\|(u, v)\|^{2}=\int_{\mathbb{R}^{N}}\left(|\nabla u|^{2}+|\nabla v|^{2}+u^{2}+v^{2}\right) \mathrm{d} x .
$$

O funcional associado ao problema $(\mathbb{S})$ é definido por

$$
I(u, v)=\frac{1}{2}\|(u, v)\|^{2}-\lambda \int_{\mathbb{R}^{N}} u v \mathrm{~d} x-\int_{\mathbb{R}^{N}}(1+a(x)) F(u, v) \mathrm{d} x,
$$

e sua derivada

$$
\begin{aligned}
I^{\prime}(u, v)(\varphi, \psi)= & \int_{\mathbb{R}^{N}}(\nabla u \nabla \varphi+\nabla v \nabla \psi+u \varphi+v \psi) \mathrm{d} x-\lambda \int_{\mathbb{R}^{N}}(v \varphi+u \psi) \mathrm{d} x \\
& -\int_{\mathbb{R}^{N}}(1+a(x)) \nabla F(u, v)(\varphi, \psi) \mathrm{d} x .
\end{aligned}
$$

Trabalharemos com a variedade de Nehari definida por

$$
\mathcal{N}:=\left\{(u, v) \in \mathbb{R}^{N} \times \mathbb{R}^{N} \backslash\{(0,0)\}: J(u, v)=0\right\}
$$

cujo funcional associado é

$$
J(u, v)=I^{\prime}(u, v)(u, v)=\|(u, v)\|^{2}-2 \lambda \int_{\mathbb{R}^{N}} u v \mathrm{~d} x-\int_{\mathbb{R}^{N}}(1+a(x)) \nabla F(u, v)(u, v) \mathrm{d} x
$$

Consideramos também o nível de energia do funcional $I$ definido por

$$
m:=\inf _{(u, v) \in \mathcal{N}} I(u, v)
$$


Decorre de $\left(a_{1}\right)$ que o problema limite associado ao sistema $(\mathbb{S})$ é

$$
\left(\mathbb{S}_{\infty}\right)\left\{\begin{array}{l}
-\Delta u+u=\frac{u\left(u^{2}+v^{2}\right)}{1+s\left(u^{2}+v^{2}\right)}+\lambda v, \quad \mathbb{R}^{N} \\
-\Delta v+v=\frac{v\left(u^{2}+v^{2}\right)}{1+s\left(u^{2}+v^{2}\right)}+\lambda u, \quad \mathbb{R}^{N} \\
u, v \in H^{1}\left(\mathbb{R}^{N}\right) .
\end{array}\right.
$$

Analogamente para o sistema $\left(\mathbb{S}_{\infty}\right)$, podemos definir $I_{\infty}, J_{\infty}, \mathcal{N}_{\infty}$ e $m_{\infty}$, respectivamente.

Note que temos para quaisquer $u, v$ em $H^{1}\left(\mathbb{R}^{N}\right)$

$$
\begin{aligned}
I(u, v) & =\frac{1}{2}\|(u, v)\|^{2}-\lambda \int_{\mathbb{R}^{N}} u v \mathrm{~d} x-\int_{\mathbb{R}^{N}}(1+a(x)) F(u, v) \mathrm{d} x \\
& =I_{\infty}(u, v)-\int_{\mathbb{R}^{N}} a(x) F(u, v) \mathrm{d} x .
\end{aligned}
$$

A hipótese $0<s<1 /(1+\lambda)$ é suficiente para que a identidade de Pohozaev possa ser satisfeita por pares $(u, v) \neq(0,0)$. De fato, considere $G(u, v)=F(u, v)+\lambda u v-\frac{u^{2}}{2}-\frac{v^{2}}{2}$. Desejamos mostrar que a identidade de Pohozaev associado ao sistema $\left(\mathbb{S}_{\infty}\right)$

$$
\frac{N-2}{2}\|(\nabla u, \nabla v)\|_{L^{2}}^{2}=N \int_{\mathbb{R}^{N}} G(u, v) \mathrm{d} x
$$

pode ser válida para algum $(u, v) \neq(0,0)$. Observe que se $0<\lambda<\left(\frac{1}{s}-1\right)$, então

$$
\begin{aligned}
\int_{\mathbb{R}^{N}} G(u, v) \mathrm{d} x & =\int_{\mathbb{R}^{N}}\left(\frac{u^{2}+v^{2}}{2 s}-\frac{1}{2 s^{2}} \ln \left(1+s\left(u^{2}+v^{2}\right)\right)+\lambda u v-\frac{u^{2}}{2}-\frac{v^{2}}{2}\right) \mathrm{d} x \\
& =\int_{\mathbb{R}^{N}}\left[\frac{u^{2}+v^{2}}{2}\left(\frac{1}{s}-1\right)+\lambda u v-\frac{1}{2 s^{2}} \ln \left(1+s\left(u^{2}+v^{2}\right)\right)\right] \mathrm{d} x \\
& =\int_{\mathbb{R}^{N}} \frac{M}{2}\left[u^{2}+v^{2}+\frac{2 \lambda}{M} u v\right] \mathrm{d} x-\int_{\mathbb{R}^{N}}\left[\frac{1}{2 s^{2}} \ln \left(1+s\left(u^{2}+v^{2}\right)\right)\right] \mathrm{d} x>0
\end{aligned}
$$

onde $M=\left(\frac{1}{s}-1\right)$.

Observação 2.1. Pelo Teorema 2.1 em [28] existe solução positiva radial de energia mínima (ground state solution) do problema $\left(\mathbb{S}_{\infty}\right)$. Por [14], se $(u, v)$ é positiva segue que $(u, v)$ é uma solução radialmente simétrica e estritamente decrescente para $\left(\mathbb{S}_{\infty}\right)$. No que segue, iremos denotar uma solução ground state positiva por $\left(w_{1}, w_{2}\right)$. Nós enfatizamos em todo trabalho que ser uma solução positiva significa ser positiva em cada coordenada do vetor $(u, v)$.

\subsection{Decaimento das soluções do problema limite}

Observe que quando $u=v=z \operatorname{em}\left(\mathbb{S}_{\infty}\right)$, o sistema se reduz à equação

$$
-\Delta z+(1-\lambda) z=h(z), \quad \mathbb{R}^{N},
$$


onde $h(z)=\frac{2 z^{3}}{1+2 s z^{2}}$. Uma condição necessária para que esta equação tenha solução não trivial é

$$
\frac{1}{s}=\lim _{z \rightarrow+\infty} \frac{h(z)}{z}>1-\lambda
$$

Note que temos

$$
0<s<\frac{1}{1+\lambda}<\frac{1}{1-\lambda}
$$

Considere a equacão

$$
-\Delta z=\psi(z):=h(z)-(1-\lambda) z
$$

Note que,

i) $\psi(0)=0$;

ii) $\psi^{\prime}(z)=\lambda-1+h^{\prime}(z) \cdot \operatorname{Assim}, \psi^{\prime}(0)=\lambda-1<0$.

Com os resultados em $(i),(i i)$ podemos utilizar a observação do Teorema 2 em [25] para obter que

$$
\lim _{|x| \rightarrow \infty} z(x)|x|^{\frac{N-1}{2}} e^{\sqrt{1-\lambda}|x|} \in(0,+\infty)
$$

A solução $(z, z)$ é positiva, radial com decaimento exponencial, porém não sabemos se é solução ground state.

Nosso objetivo agora é mostrar que se o par $(u, v)$ é solução de $\left(\mathbb{S}_{\infty}\right)$, então mantém-se o mesmo decaimento por meio do seguinte teorema

Teorema 2.1. Uma solução positiva de energia minima $\left(w_{1}, w_{2}\right)$ do problema $\left(\mathbb{S}_{\infty}\right)$ satisfaz

$$
\lim _{|x| \rightarrow \infty} w_{1}(x)|x|^{\frac{N-1}{2}} e^{\sqrt{1-\lambda}|x|}=\mu_{1}
$$

$e$

$$
\lim _{|x| \rightarrow \infty} w_{2}(x)|x|^{\frac{N-1}{2}} e^{\sqrt{1-\lambda}|x|}=\mu_{2}
$$

$\operatorname{com} \mu_{1}, \mu_{2} \in(0,+\infty)$.

No resultado seguinte iremos mostrar um decaimento para o problema $\left(\mathbb{S}_{\infty}\right)$ que ainda não é o decaimento "ideal" que almejamos. Isto será útil para obtermos o decaimento ideal, exato. A prova é semelhante a encontrada no Teorema 2.2 em [21] ou Proposição 4.4 em [43].

Lema 2.2. Se $(u, v)$ é solução positiva do problema $\left(\mathbb{S}_{\infty}\right)$, então existe uma constante $C>0$ tal que

$$
0<u(x), v(x) \leq C|x|^{-\frac{N-1}{2}} e^{-\theta|x|}
$$

para $|x|>R, R>0$ suficientemente grande $, \theta=\sqrt{1-\lambda-\varepsilon}, N \geq 3$ e $\varepsilon>0$ é uma constante dada.

Demonstração: Inicialmente, vamos reescrever o problema $\left(\mathbb{S}_{\infty}\right)$ na forma matricial, isto é , $U \in H$,

$$
L(U)+A U=\mathcal{F}
$$

onde

$$
U:=\left(\begin{array}{l}
u \\
v
\end{array}\right), L:=\left(\begin{array}{cc}
-\Delta & 0 \\
0 & -\Delta
\end{array}\right), \quad A:=\left(\begin{array}{cc}
1 & -\lambda \\
-\lambda & 1
\end{array}\right) \text { e } \mathcal{F}:=\left(\begin{array}{l}
f(u, v) \\
g(u, v)
\end{array}\right)
$$


Defina agora a função auxiliar

$$
\varphi(x)=\frac{M}{R^{-\frac{N-1}{2}}}|x|^{-\frac{N-1}{2}} e^{-\theta(|x|-R)}=\bar{M}(R)|x|^{-\frac{N-1}{2}} e^{-\theta|x|}
$$

onde $M=u(R)+v(R),|x|=R$.

Vamos calcular o Laplaciano de $\varphi$. Inicialmente, note que

$$
\begin{aligned}
\frac{\partial \varphi(x)}{\partial x_{i}} & =\bar{M}\left(-\frac{N-1}{2}\right)|x|^{-\frac{N-1}{2}-1} \frac{x_{i}}{|x|} e^{-\theta|x|}+\bar{M}|x|^{-\frac{N-1}{2}} e^{-\theta|x|}\left(-\frac{\theta x_{i}}{|x|}\right) \\
& =\varphi(x) \frac{x_{i}}{|x|}\left(-\frac{N-1}{2|x|}-\theta\right) .
\end{aligned}
$$

Derivando a função $\varphi$ mais uma vez, temos

$$
\begin{aligned}
\frac{\partial^{2} \varphi(x)}{\partial x_{i}^{2}}= & \varphi(x) \frac{x_{i}^{2}}{|x|^{2}}\left(-\frac{N-1}{2|x|}-\theta\right)^{2}+\varphi(x)\left(\frac{1}{|x|}-\frac{x_{i}^{2}}{|x|^{2}}\right)\left(-\frac{N-1}{2|x|}-\theta\right) \\
& +\varphi(x) \frac{x_{i}}{|x|} \frac{(N-1) x_{i}}{2|x|^{3}} .
\end{aligned}
$$

Agora vamos somar (2.8) em $i$, de 1 a $N$. Segue que

$$
\begin{aligned}
\Delta \varphi(x) & =\varphi(x)\left(-\frac{N-1}{2|x|}-\theta\right)^{2}+\varphi(x)\left(\frac{N-1}{|x|}\right)\left(-\frac{N-1}{2|x|}-\theta\right)+\varphi(x) \frac{(N-1)}{2|x|^{2}} \\
& =\varphi(x)\left\{\frac{(N-1)^{2}}{4|x|^{2}}+\frac{(N-1) \theta}{|x|}+\theta^{2}-\frac{(N-1)^{2}}{2|x|^{2}}-\frac{(N-1) \theta}{|x|}+\frac{(N-1)}{2|x|^{2}}\right\} \\
& =\varphi(x)\left\{\frac{(N-1)^{2}}{4|x|^{2}}+\theta^{2}-\frac{2(N-1)^{2}}{4|x|^{2}}+\frac{2(N-1)}{4|x|^{2}}\right\} \\
& =\varphi(x)\left\{\theta^{2}+\frac{(N-1)}{4|x|^{2}}(N-1-2 N+2+2)\right\} \\
& =\varphi(x)\left\{\theta^{2}-\frac{(N-1)}{4|x|^{2}}(N-3)\right\} .
\end{aligned}
$$

Como $(u, v)$ é solução de $\left(\mathbb{S}_{\infty}\right)$, então $u(x), v(x) \rightarrow 0$, quando $|x| \rightarrow+\infty$. Assim, dado $\varepsilon>0$ tal que $1-\lambda \geq \frac{4 \varepsilon}{3}$ e $\theta=\sqrt{1-\lambda-\varepsilon}$, existe um $\delta=\delta(\varepsilon)$ tal que se $u^{2}+v^{2}<\delta$ tem-se

$$
\frac{f(u, v)}{u}=\frac{g(u, v)}{v}<\varepsilon .
$$

Considere $L>R$ e o conjunto aberto

$$
\Omega_{L}:=\left\{x \in \mathbb{R}^{N}: R<|x|<L ; u(x)+v(x)>\varphi(x)\right\}
$$

Desejando mostrar que o conjunto $\Omega_{L}$ é vazio. Suponha então, por contradição, que $\Omega_{L} \neq \emptyset$. Note 
que, para todo $|x| \geq R$, temos por (2.9), (2.10) e $N \geq 3$ que

$$
\begin{aligned}
\Delta(\varphi-u-v) & =\varphi\left\{\theta^{2}-\frac{(N-1)}{4|x|^{2}}(N-3)\right\}-\Delta u-\Delta v \\
& <\varphi\left\{\theta^{2}-\frac{(N-1)}{4|x|^{2}}(N-3)\right\}-u+\lambda v+\varepsilon u-v+\lambda u+\varepsilon v \\
& \leq \varphi \theta^{2}-(1-\lambda-\varepsilon) u-(1-\lambda-\varepsilon) v \\
& \leq \theta^{2}(\varphi-u-v)<0 \text { em } \Omega_{L}
\end{aligned}
$$

Desde que $\Omega_{L}$ é limitado, $\Delta(\varphi-u-v)<0$ em $\Omega_{L}$, pelo Princípio do Máximo, temos por $(2.11)$ que

$$
\begin{aligned}
\varphi(x)-u(x)-v(x) & \geq \min \left\{(\varphi-u-v)(x): x \in \partial \Omega_{L}\right\} \\
& =\min \{\varphi(R)-u(R)-v(R), \varphi(L)-u(L)-v(L)\} \\
& \geq \min \{0, \varphi(L)-u(L)-v(L)\}, \forall x \in \Omega_{L} .
\end{aligned}
$$

Quando $L \rightarrow+\infty$, observe que $u, v, \varphi \rightarrow 0, \operatorname{logo}$ temos $\varphi(x)-u(x)-v(x) \geq 0$ para todo $x \in \Omega_{L}$ tal que $|x| \geq R$, implicando em contradição. Portanto, $\Omega_{L}$ é vazio e finalizamos a prova do lema.

Vamos adaptar os argumentos de [37] para o sistema a fim de obter os decaimentos exponenciais exatos. No que segue, vamos tomar $\gamma=0$ na Proposição 6.1 em [37]. Observe no Teorema 2.1 o fato de $\mu_{1}$ e $\mu_{2}$ serem constantes positivas significa que tem-se o decaimento a zero das soluções $w_{1}$ e $w_{2}$ em ordem exata e será fundamental em argumento posterior.

No que segue, denotaremos $\left(w_{1}, w_{2}\right)$ solução positiva radial de $\left(\mathbb{S}_{\infty}\right)$, obtida em [28], em que $I_{\infty}\left(w_{1}, w_{2}\right)=$ $m_{\infty}$

Lema 2.3. Sejam $N \geq 3, \quad \rho>0$ e $0<\lambda<1$. Então existem funções radiais não negativas u, $v$ : $\mathbb{R}^{N} \backslash B_{\rho}(0) \rightarrow \mathbb{R}$ do sistema linear

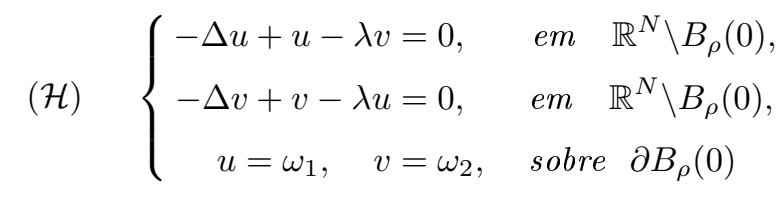

e, para algum $\rho_{0} \in(\rho,+\infty)$,

$$
\lim _{|x| \rightarrow \infty} u(x)|x|^{\frac{N-1}{2}} e^{\int_{\rho_{0}}^{|x|} \sqrt{1-\lambda} \mathrm{d} t}=\sigma_{1}>0
$$

e

$$
\lim _{|x| \rightarrow \infty} v(x)|x|^{\frac{N-1}{2}} e^{\int_{\rho_{0}}^{|x|} \sqrt{1-\lambda} \mathrm{d} t}=\sigma_{2}>0 .
$$

Demonstração: A prova de existência de solução para $(\mathcal{H})$ é consequência da teoria de EDO lineares, entretanto iremos incluí-la com detalhes para efeito de completude. Primeiramente, iremos mostrar que 
o problema $(\mathcal{H})$ possui uma subsolução e supersolução. Depois iremos usar [19] para garantir a existência de solução entre essa subsolução e supersolução .

Considere $0 \leq \beta<1, t>\rho$ e $\tau \in \mathbb{R}$. Defina a função

$$
\phi_{\tau}(t)=-\frac{N-1}{2 t}-\sqrt{1-\lambda}+\frac{\tau}{t^{1+\beta}}
$$

e para $x \in \mathbb{R}^{N} \backslash \bar{B}_{\rho}(0)$ considere

$$
\psi_{\tau}(x)=\left(\begin{array}{c}
\Phi_{\tau}(x) \\
\Phi_{\tau}(x)
\end{array}\right):=\left(\begin{array}{c}
e_{\rho}^{\int_{\rho}^{|x|} \phi_{\tau}(t) \mathrm{d} t} \\
\int_{\rho}^{|x|} \phi_{\tau}(t) \mathrm{d} t
\end{array}\right)
$$

Pela regra da cadeia tem-se

$$
\frac{\partial \Phi_{\tau}(x)}{\partial x_{i}}=\Phi_{\tau}(x) \phi_{\tau}(|x|) \frac{x_{i}}{|x|} .
$$

Derivando novamente temos

$$
\begin{aligned}
\frac{\partial^{2} \Phi_{\tau}(x)}{\partial x_{i}^{2}} & =\frac{\partial \Phi_{\tau}(x)}{\partial x_{i}} \phi_{\tau}(|x|) \frac{x_{i}}{|x|}+\Phi_{\tau}(x) \phi_{\tau}^{\prime}(|x|) \frac{x_{i}^{2}}{|x|^{2}}+\Phi_{\tau}(x) \phi_{\tau}(|x|)\left(\frac{1}{|x|}-\frac{x_{i}^{2}}{|x|^{3}}\right) \\
& =\Phi_{\tau}(x)\left[\phi_{\tau}(|x|) \frac{x_{i}}{|x|}\right]^{2}+\Phi_{\tau}(x) \phi_{\tau}^{\prime}(|x|) \frac{x_{i}^{2}}{|x|^{2}}+\Phi_{\tau}(x) \phi_{\tau}(|x|)\left(\frac{1}{|x|}-\frac{x_{i}^{2}}{|x|^{3}}\right) \\
& =\Phi_{\tau}(x)\left[\phi_{\tau}^{2}(|x|) \frac{x_{i}^{2}}{|x|^{2}}+\phi_{\tau}^{\prime}(|x|) \frac{x_{i}^{2}}{|x|^{2}}+\phi_{\tau}(|x|)\left(\frac{1}{|x|}-\frac{x_{i}^{2}}{|x|^{3}}\right)\right] .
\end{aligned}
$$

Somando em $i$ de 1 a $N$ temos

$$
-\Delta \Phi_{\tau}(x)=\Phi_{\tau}(x)\left[-\phi_{\tau}^{2}(|x|)-\phi_{\tau}^{\prime}(|x|)-\phi_{\tau}(|x|)\left(\frac{N-1}{|x|}\right)\right] .
$$

Por outro lado, derivando (2.14) em $t$ obtemos

$$
\phi_{\tau}^{\prime}(t)=\frac{N-1}{2 t^{2}}-\frac{\tau(1+\beta)}{t^{2+\beta}} .
$$

Segue para $t=|x| \geq \rho$ que

$$
\phi_{\tau}^{\prime}(|x|)=\frac{N-1}{2|x|^{2}}-\frac{\tau(1+\beta)}{|x|^{2+\beta}} .
$$


Agora, substituindo (2.14) e (2.17) em (2.16) temos

$$
\begin{aligned}
-\Delta \Phi_{\tau}(x)= & \Phi_{\tau}(x)\left[-\left(\frac{N-1}{2|x|}-\sqrt{1-\lambda}+\frac{\tau}{|x|^{1+\beta}}\right)^{2}-\frac{N-1}{2|x|^{2}}+\frac{\tau(1+\beta)}{|x|^{2+\beta}}\right. \\
& \left.+\frac{(N-1)(N-1)}{2|x|^{2}}+\frac{\sqrt{1-\lambda}(N-1)}{|x|}-\frac{\tau(N-1)}{|x|^{2+\beta}}\right] \\
= & \Phi_{\tau}(x)\left[-\left(\frac{N-1}{2|x|}\right)^{2}-(1-\lambda)-\frac{\tau^{2}}{|x|^{2+2 \beta}}-\frac{(N-1) \sqrt{1-\lambda}}{|x|}+\frac{\tau(N-1)}{|x|^{2+\beta}}\right. \\
& +\frac{2 \sqrt{1-\lambda} \tau}{|x|}-2 \frac{(N-1)}{4|x|^{2}}+\frac{\tau(1+\beta)}{|x|^{2+\beta}} \\
& \left.+2 \frac{(N-1)(N-1)}{4|x|^{2}}+\frac{\sqrt{1-\lambda}(N-1)}{|x|}-\frac{\tau(N-1)}{|x|^{2+\beta}}\right] \\
= & \Phi_{\tau}(x)\left[\frac{(N-1)(N-3)}{4|x|^{2}}-(1-\lambda)-\frac{\tau^{2}}{|x|^{2+2 \beta}}+\frac{\tau(1+\beta)}{2|x|^{2+2 \beta}}+\frac{2 \tau \sqrt{1-\lambda}}{|x|^{1+\beta}}\right] .
\end{aligned}
$$

Segue que

$$
-\Delta \Phi_{\tau}(x)+(1-\lambda) \Phi_{\tau}(x)=\frac{\Phi_{\tau}(x)}{|x|^{1+\beta}}\left[2 \tau \sqrt{1-\lambda}+\frac{(N-1)(N-3)}{4|x|^{1-\beta}}-\frac{\tau^{2}}{|x|^{1+\beta}}+\frac{\tau(1+\beta)}{|x|^{1}}\right] .
$$

Reescrevendo de forma mais conveniente temos

$$
-\Delta \Phi_{\tau}(x)+(1-\lambda) \Phi_{\tau}(x)=\Phi_{\tau}(x)\left[\frac{2 \tau \sqrt{1-\lambda}}{|x|^{1+\beta}}+\frac{\pi_{\tau}(x)}{|x|^{1+\beta}}\right],
$$

onde $\pi_{\tau}(x): \mathbb{R}^{N} \backslash B_{\rho}(0) \rightarrow \mathbb{R}$ é dada por

$$
\pi_{\tau}(x)=\frac{(N-1)(N-3)}{4|x|^{1-\beta}}-\frac{\tau^{2}}{|x|^{1+\beta}}+\frac{\tau(1+\beta)}{|x|} .
$$

Como $\beta<1$ segue que

$$
\lim _{|x| \rightarrow+\infty} \pi_{\tau}(x)=0
$$

Assim, podemos escolher $\rho_{0}$ suficientemente grande tal que para todo $\rho \geq \rho_{0}$, se $|x| \geq \rho$ temos $\pi_{\tau}(x)$ pequeno de tal forma que escolhendo $\bar{\tau}>0$ e $\underline{\tau}<0$, segue por (2.18) que existem funções

$$
\psi_{\underline{\tau}}(x)=\left(\begin{array}{c}
\Phi_{\underline{\tau}}(x) \\
\Phi_{\underline{\tau}}(x)
\end{array}\right) \quad \text { e } \quad \psi_{\bar{\tau}}(x)=\left(\begin{array}{c}
\Phi_{\bar{\tau}}(x) \\
\Phi_{\bar{\tau}}(x)
\end{array}\right)
$$

subsolução e supersolução do problema $(\mathcal{H})$, ou seja, em termos de matrizes

$$
\left(\begin{array}{cc}
-\Delta & 0 \\
0 & -\Delta
\end{array}\right)\left(\begin{array}{c}
\Phi_{\bar{\tau}}(x) \\
\Phi_{\bar{\tau}}(x)
\end{array}\right)+\left(\begin{array}{cc}
1 & -\lambda \\
-\lambda & 1
\end{array}\right)\left(\begin{array}{l}
\Phi_{\bar{\tau}}(x) \\
\Phi_{\bar{\tau}}(x)
\end{array}\right) \gg\left(\begin{array}{l}
0 \\
0
\end{array}\right)
$$

e

$$
\left(\begin{array}{cc}
-\Delta & 0 \\
0 & -\Delta
\end{array}\right)\left(\begin{array}{c}
\Phi_{\underline{\tau}}(x) \\
\Phi_{\underline{\tau}}(x)
\end{array}\right)+\left(\begin{array}{cc}
1 & -\lambda \\
-\lambda & 1
\end{array}\right)\left(\begin{array}{l}
\Phi_{\underline{\tau}}(x) \\
\Phi_{\underline{\tau}}(x)
\end{array}\right) \ll\left(\begin{array}{l}
0 \\
0
\end{array}\right)
$$


onde o símbolos $\ll \mathrm{e} \gg$ significam $\leq \mathrm{e} \geq$, respectivamente, em cada linha do sistema.

Resta mostrar que existe um vetor solução $Z=\left(\begin{array}{l}u \\ v\end{array}\right)$ de $(\mathcal{H})$ satisfazendo

$$
\left(\begin{array}{c}
\Phi_{\underline{\tau}}(x) \\
\Phi_{\underline{\tau}}(x)
\end{array}\right) \ll Z \ll\left(\begin{array}{c}
\Phi_{\bar{\tau}}(x) \\
\Phi_{\bar{\tau}}(x)
\end{array}\right) .
$$

Vamos utilizar o método de redução de ordem. Desde que as soluções do sistema são radiais por $([14])$, vamos reescrever o sistema $(\mathcal{H})$ da seguinte forma

$$
\left\{\begin{array}{rlrl}
-\Delta u(r)+u(r) & =\lambda v(r), & & r \in(\rho,+\infty), \\
-\Delta v(r)+v(r) & =\lambda u(r), & r \in(\rho,+\infty), \\
u(\rho) & =\omega_{1}(\rho), & v(\rho)=\omega_{2}(\rho) .
\end{array}\right.
$$

Como $u$ e $v$ são radiais, então $(\mathcal{H})$ é dado por

$$
\left(\mathcal{H}_{r}\right)\left\{\begin{aligned}
u^{\prime \prime}+\frac{N-1}{r} u^{\prime}-u(r) & =-\lambda v(r), \quad r \in(\rho,+\infty), \\
v^{\prime \prime}+\frac{N-1}{r} v^{\prime}-v(r) & =-\lambda u(r), \quad r \in(\rho,+\infty), \\
u(\rho) & =\omega_{1}(\rho), \quad v(\rho)=\omega_{2}(\rho) .
\end{aligned}\right.
$$

Consideremos a seguinda mudança de variáveis:

$$
x_{1}=u ; \quad x_{2}=x_{1}^{\prime} ; \quad x_{3}=v ; \quad x_{4}=x_{3}^{\prime}
$$

com condições iniciais

$$
x_{1}(\rho)=\omega_{1}(\rho) ; \quad x_{3}(\rho)=\omega_{2}(\rho) ; \quad x_{2}<0 ; \quad x_{4}<0
$$

Assim, obtemos o sistema de EDO linear

$$
\left(\mathcal{H}_{R}\right) \quad\left\{\begin{aligned}
x_{1}^{\prime} & =x_{2}, \\
x_{2}^{\prime} & =x_{1}+\frac{1-N}{r} x_{2}-\lambda x_{3}, \\
x_{3}^{\prime} & =x_{4}, \\
x_{4}^{\prime} & =-\lambda x_{1}+x_{3}+\frac{1-N}{r} x_{3} .
\end{aligned}\right.
$$

Ou seja,

$$
X^{\prime}=\left(\begin{array}{cccc}
0 & 1 & 0 & 0 \\
1 & \frac{1-N}{r} & -\lambda & 0 \\
0 & 0 & 0 & 1 \\
-\lambda & 0 & 1 & \frac{1-N}{r}
\end{array}\right)\left(\begin{array}{c}
x_{1} \\
x_{2} \\
x_{3} \\
x_{4}
\end{array}\right)
$$

Podemos olhar o sistema na forma $X^{\prime}=A(r) X$ onde $A(r) X=f\left(r, x_{1}, x_{2}, x_{3}, x_{4}\right)$. Como os coeficientes da matriz $A$ são limitados e contínuos para $r>\rho$, podemos usar o Teorema 5.2 em [19] para garantir que existe solução $(u(x), v(x))=(u(r), v(r))$ para qualquer $r \in(\rho,+\infty)$. Feito isto, observe que, por 
construção temos que

$$
\Phi_{\underline{\tau}}(x) \leq u(x), v(x) \leq \Phi_{\bar{\tau}}(x) .
$$

A fim de mostrar o decaimento das soluções $u$ e $v$, nós precisamos de dois resultados auxiliares.

Afirmação 2.4. As seguintes afirmativas são verdadeiras:

$$
\lim _{|x| \rightarrow+\infty} \frac{\Phi_{\bar{\tau}}(x)}{\Phi_{\underline{\tau}}(x)}<+\infty
$$

$e$

$$
\lim _{|x| \rightarrow+\infty} \Phi_{\underline{\tau}}(x)|x|^{\frac{N-1}{2}} e^{\int_{\rho}^{|x|} \sqrt{1-\lambda} \mathrm{d} t} \in(0,+\infty) .
$$

Demonstração: Começamos observando que, por (2.14),

$$
\phi_{\bar{\tau}}(t)-\phi_{\underline{\tau}}(t)=-\frac{N-1}{2 t}-\sqrt{1-\lambda}+\frac{\bar{\tau}}{t^{1+\beta}}+\frac{N-1}{2 t}+\sqrt{1-\lambda}-\frac{\underline{\tau}}{t^{1+\beta}}=\frac{\bar{\tau}-\underline{\tau}}{s^{1+\beta}} .
$$

Integrando de $\rho$ a $|x|$ temos

$$
\begin{aligned}
\int_{\rho}^{|x|}\left(\phi_{\bar{\tau}}(t)-\phi_{\underline{\tau}}(t)\right) \mathrm{d} t & =\int_{\rho}^{|x|} \frac{\bar{\tau}-\underline{\tau}}{s^{1+\beta}} \mathrm{d} t=(\bar{\tau}-\underline{\tau}) \int_{\rho}^{|x|} t^{-(1+\beta)} \mathrm{d} t \\
& =\left.(\bar{\tau}-\underline{\tau})\left\{-\frac{1}{t^{\beta}}\right\}\right|_{t=\rho} ^{|x|}=(\bar{\tau}-\underline{\tau})\left\{-\frac{1}{|x|^{\beta}}+\frac{1}{\rho^{\beta}}\right\}
\end{aligned}
$$

Segue então que

$$
\begin{aligned}
\lim _{|x| \rightarrow+\infty} \frac{\Phi_{\bar{\tau}}(x)}{\Phi_{\underline{\tau}}(x)} & =\lim _{|x| \rightarrow+\infty} e^{\int_{\rho}^{|x|}\left(\phi_{\bar{\tau}}(t)-\phi_{\underline{\tau}}(t)\right) \mathrm{d} t} \\
& =\lim _{|x| \rightarrow+\infty} e^{\left\{(\bar{\tau}-\underline{\tau})\left\{-\frac{1}{|x|^{\beta}}+\frac{1}{\rho^{\beta}}\right\}\right\}} \\
& =e^{\left\{(\bar{\tau}-\underline{\tau})\left\{\frac{1}{\rho^{\beta}}\right\}\right\}<+\infty .}
\end{aligned}
$$

Assim, mostramos (2.23). Por outro lado, por (2.14) temos 


$$
\begin{aligned}
& \Phi_{\underline{\tau}}(x)|x|^{\frac{N-1}{2}} e^{\int_{\rho}^{|x|} \sqrt{1-\lambda} \mathrm{d} t}=\left(e^{\left.\int_{\rho}^{|x|} \phi_{\underline{\tau}}(t) \mathrm{d} t\right)}\right)\left(|x|^{\frac{N-1}{2}} e^{\int_{\rho}^{|x|} \sqrt{1-\lambda} \mathrm{d} t}\right)
\end{aligned}
$$

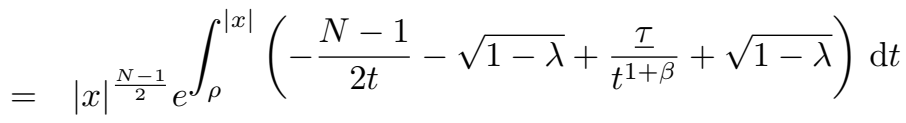

$$
\begin{aligned}
& =|x|^{\frac{N-1}{2}} e^{\int_{\rho}^{|x|}}\left(-\frac{N-1}{2 t}+\frac{\underline{\tau}}{t^{1+\beta}}\right) \mathrm{d} t \\
& =|x|^{\frac{N-1}{2}} e^{\left(-\frac{N-1}{2} \ln |x|+\frac{N-1}{2} \ln \rho-\frac{\underline{\tau}}{|x|^{\beta}}+\frac{\underline{\tau}}{\rho^{\beta}}\right)} \\
& =|x|^{\frac{N-1}{2}}|x|^{-\frac{N-1}{2}} e^{\left(\frac{N-1}{2} \ln \rho-\frac{\underline{\tau}}{|x|^{\beta}}+\frac{\underline{\tau}}{\rho^{\beta}}\right)} \\
& =e^{\left(\frac{N-1}{2} \ln \rho-\frac{\tau}{|x|^{\beta}}+\frac{\tau}{\rho^{\beta}}\right)} \text {. }
\end{aligned}
$$

Aplicando o limite obtemos

$$
\lim _{|x| \rightarrow \infty} \Phi_{\underline{\tau}}(x)|x|^{\frac{N-1}{2}} e^{\int_{\rho}^{|x|} \sqrt{1-\lambda} \mathrm{d} t}=e^{\left(\frac{N-1}{2} \ln \rho+\frac{\underline{\tau}}{\rho^{\beta}}\right)} .
$$

Portanto, concluímos (2.24).

Para concluirmos o lema e obtermos $(2.12)$ e $(2.13)$, podemos utilizar $(2.22),(2.23)$ e (2.24) e obter que

$$
\begin{aligned}
0 & <\lim _{|x| \rightarrow \infty} u(x)|x|^{\frac{N-1}{2}} e^{\int_{\rho}^{|x|} \sqrt{1-\lambda} \mathrm{d} t} \\
& =\left(\lim _{|x| \rightarrow \infty} \frac{u(x)}{\Phi_{\underline{\tau}}(x)}\right)\left(\lim _{|x| \rightarrow \infty} \Phi_{\underline{\tau}}(x)|x|^{\frac{N-1}{2}} e^{\left.\int_{\rho}^{|x|} \sqrt{1-\lambda} \mathrm{d} t\right)}\right) \\
& \leq\left(\lim _{|x| \rightarrow \infty} \frac{\Phi_{\bar{\tau}}(x)}{\Phi_{\underline{\tau}}(x)}\right)\left(\lim _{|x| \rightarrow \infty} \Phi_{\underline{\tau}}(x)|x|^{\frac{N-1}{2}} e^{\int_{\rho}^{|x|} \sqrt{1-\lambda} \mathrm{d} t}\right) \in(0,+\infty) .
\end{aligned}
$$

Similarmente, $(2.25)$ vale para $v(x)$.

Prova do Teorema 2.1. Primeiramente denotamos uma solução positiva radial de $\left(\mathbb{S}_{\infty}\right)$ por

$$
U(x):=\left(\begin{array}{l}
w_{1}(x) \\
w_{2}(x)
\end{array}\right)
$$


Vamos considerar dois problemas auxiliares, a saber: sejam $H_{0}, H^{0} \in C^{2}\left(\mathbb{R}^{N} \backslash B_{\rho}(0)\right) \times C^{2}\left(\mathbb{R}^{N} \backslash B_{\rho}(0)\right)$ satisfazendo

$$
\left(\mathcal{H}_{0}\right) \quad\left\{\begin{array}{rlrl}
-\Delta H_{0}+A H_{0} & =0, & \mathbb{R}^{N} \backslash B_{\rho}(0), \\
H_{0}(x) & =U(x), \quad \Gamma=\partial B_{\rho}(0), \\
\lim _{|x| \rightarrow+\infty} H_{0}(x) & =0, & &
\end{array}\right.
$$

e

$$
\left(\mathcal{H}^{0}\right) \quad\left\{\begin{array}{rlrl}
-\Delta H^{0}+W H^{0} & =0, & & \mathbb{R}^{N} \backslash B_{\rho}(0), \\
H^{0}(x) & =U(x), & \Gamma=\partial\left(B_{\rho}(0),\right. \\
\lim _{|x| \rightarrow+\infty} H^{0}(x) & =0, & &
\end{array}\right.
$$

onde $H_{0}(x):=\left(\begin{array}{c}h_{0,1}(x) \\ h_{0,2}(x)\end{array}\right), H^{0}(x):=\left(\begin{array}{c}h^{0,1}(x) \\ h^{0,2}(x)\end{array}\right), A:=\left(\begin{array}{cc}1 & -\lambda \\ -\lambda & 1\end{array}\right), 0:=\left(\begin{array}{l}0 \\ 0\end{array}\right), W(x):=$ $\left(\begin{array}{cc}1-c e^{-\theta|x|} & -\lambda \\ -\lambda & 1-c e^{-\theta|x|}\end{array}\right)$ onde $c$ é uma constante positiva e $\theta>0$ escolhido no Lema 2.2. Note que $H_{0}$ satisfaz o Lema 2.3, então existe $\mu_{0}>0$ tal que

$$
\lim _{|x| \rightarrow \infty} H_{0}(x)|x|^{\frac{N-1}{2}} e^{\int_{\rho}^{|x|} \sqrt{1-\lambda} \mathrm{d} t}=\left(\begin{array}{c}
\mu_{0} \\
\mu_{0}
\end{array}\right)
$$

Similarmente como feito nos passos de (2.14) a (2.20) tomando $\phi_{\tau}(t)=-\frac{N-1}{2 t}-W(t)+\frac{\tau}{t^{1+\beta}}$ em vez de $\phi_{\tau}(t)=-\frac{N-1}{2 t}-\sqrt{1-\lambda}+\frac{\tau}{t^{1+\beta}}$ em $(2.14)$, com $W(t)=\sqrt{1-\lambda-c e^{-\theta t}}$, desde que para algum $\beta>0$ tem-se $\lim _{t \rightarrow \infty} W^{\prime}(t) t^{1+\beta}=0$, veja a Proposição 6.1 em [37], obtemos

$$
\lim _{|x| \rightarrow \infty} H^{0}(x)|x|^{\frac{N-1}{2}} e^{\int_{\rho}^{|x|} \sqrt{1-\lambda-c e^{-\theta t}} \mathrm{~d} t}=\left(\begin{array}{c}
\mu^{0} \\
\mu^{0}
\end{array}\right)
$$

com $\mu^{0} \in(0,+\infty)$. Ademais,

$$
\left(\begin{array}{cc}
-\Delta & 0 \\
0 & -\Delta
\end{array}\right)\left(\begin{array}{c}
\Phi_{\bar{\tau}}(x) \\
\Phi_{\bar{\tau}}(x)
\end{array}\right)+\left(\begin{array}{cc}
1-c e^{-\theta|x|} & -\lambda \\
-\lambda & 1-c e^{-\theta|x|}
\end{array}\right)\left(\begin{array}{l}
\Phi_{\bar{\tau}}(x) \\
\Phi_{\bar{\tau}}(x)
\end{array}\right) \gg\left(\begin{array}{l}
0 \\
0
\end{array}\right)
$$

e

$$
\left(\begin{array}{cc}
-\Delta & 0 \\
0 & -\Delta
\end{array}\right)\left(\begin{array}{c}
\Phi_{\underline{\tau}}(x) \\
\Phi_{\underline{\tau}}(x)
\end{array}\right)+\left(\begin{array}{cc}
1-c e^{-\theta|x|} & -\lambda \\
-\lambda & 1-c e^{-\theta|x|}
\end{array}\right)\left(\begin{array}{l}
\Phi_{\underline{\tau}}(x) \\
\Phi_{\underline{\tau}}(x)
\end{array}\right) \ll\left(\begin{array}{l}
0 \\
0
\end{array}\right) .
$$

Resta mostrar que existe um vetor solução $V=\left(\begin{array}{l}u \\ v\end{array}\right)$ de $\left(\mathcal{H}^{0}\right)$ satisfazendo

$$
\left(\begin{array}{c}
\Phi_{\underline{\tau}}(x) \\
\Phi_{\underline{\tau}}(x)
\end{array}\right) \ll V(x) \ll\left(\begin{array}{c}
\Phi_{\bar{\tau}}(x) \\
\Phi_{\bar{\tau}}(x)
\end{array}\right)
$$

Porém, é similar aos passos de (2.21) a (2.22). 
Vamos escrever o problema $\left(\mathbb{S}_{\infty}\right)$ de uma forma compacta, isto é, considere $L U+A U=\mathcal{F}$ onde

$$
L U=\left(\begin{array}{cc}
-\Delta & 0 \\
0 & -\Delta
\end{array}\right)\left(\begin{array}{l}
w_{1} \\
w_{2}
\end{array}\right) \quad \text { e } \quad \mathcal{F}=\left(\begin{array}{c}
f\left(w_{1}, w_{2}\right) \\
g\left(w_{1}, w_{2}\right)
\end{array}\right)
$$

A demonstração da estimativa exponencial para a solução do sistema $\left(\mathbb{S}_{\infty}\right)$ repousa no Princípio do Máximo em [22]. No domínio $\mathbb{R}^{N} \backslash B_{\rho}(0)$,

$$
L\left(U-H_{0}\right)=L(U)-L\left(H_{0}\right)=-A U+\mathcal{F}+A H_{0}=-A\left(U-H_{0}\right)+\mathcal{F}
$$

Como $\mathcal{F} \gg 0,-A=\left(\begin{array}{cc}-1 & \lambda \\ \lambda & -1\end{array}\right)$ e $U(x)-H_{0}(x)=0$ sobre $\Gamma$, pelo Princípio do Máximo, ver Teorema 1.1 em [22], segue que

$$
U(x) \gg H_{0}(x) \text { em } \mathbb{R}^{N} \backslash \bar{B}_{\rho}(0) .
$$

Por outro lado, observe que

$$
\begin{aligned}
L\left(H^{0}-U\right) & =L\left(H^{0}\right)-L(U) \\
& =-W H^{0}+A U-\mathcal{F} \\
& =-A\left(H^{0}-U\right)+\left(\begin{array}{cc}
c e^{-\theta|x|} & 0 \\
0 & c e^{-\theta|x|}
\end{array}\right) H^{0}-\mathcal{F} \\
& =-A\left(H^{0}-U\right)+\left(\begin{array}{c}
c e^{-\theta|x|} h^{0,1}-f\left(w_{1}, w_{2}\right) \\
c e^{-\theta|x|} h^{0,2}-g\left(w_{1}, w_{2}\right)
\end{array}\right) .
\end{aligned}
$$

Para utilizar o Princípio do Máximo, precisamos verificar que a matriz

$$
\left(\begin{array}{c}
c e^{-\theta|x|} h^{0,1}-f\left(w_{1}, w_{2}\right) \\
c e^{-\theta|x|} h^{0,2}-g\left(w_{1}, w_{2}\right)
\end{array}\right)
$$

é não negativa em cada linha. Pelo Lema 2.2, a solução vetorial do sistema

$$
L(U)+A U=\mathcal{F}
$$

tem decaimento do tipo

$$
0<w_{1}(x), w_{2}(x) \leq C|x|^{-\frac{N-1}{2}} e^{-\theta|x|}
$$

para $|x|>R, R>0$ suficientemente grande. Note que

$$
f\left(w_{1}, w_{2}\right)=\frac{w_{1}\left(w_{1}^{2}+w_{2}^{2}\right)}{1+s\left(w_{1}^{2}+w_{2}^{2}\right)} \leq w_{1}\left(w_{1}^{2}+w_{2}^{2}\right)=w_{1}^{3}+w_{2}^{2} w_{1} \leq \bar{C}|x|^{-\frac{3(N-1)}{2}} e^{-3 \theta|x|}
$$

e, similarmente, para $g\left(w_{1}, w_{2}\right)$. Consequentemente, estimando a primeira linha da matriz em (2.30), pois a segunda linha é similar, notamos que utilizando o decaimento exponencial de $H^{0}$ em $(2.27)$ temos 


$$
\begin{aligned}
& c e^{-\theta|x|} h^{0,1}-f\left(w_{1}, w_{2}\right) \\
\geq & \bar{c} e^{-\theta|x|} C|x|^{-\frac{N-1}{2}} e^{-\sqrt{1-\lambda-c e^{-\theta|x|}}|x|}-\bar{C}|x|^{-\frac{3(N-1)}{2}} e^{-3 \theta|x|} \\
\geq & C e^{-\theta|x|}|x|^{-\frac{N-1}{2}}\left(e^{-\sqrt{1-\lambda-c e^{-\theta|x|}}|x|}-|x|^{-\frac{2(N-1)}{2}} e^{-2 \theta|x|}\right) \\
\geq & C e^{-\theta|x|}|x|^{-\frac{N-1}{2}}\left(e^{-\sqrt{1-\lambda-c e^{-\theta|x|}}|x|}-|R|^{-\frac{2(N-1)}{2}} e^{-2 \theta|x|}\right) \\
\geq & C e^{-\theta|x|}|x|^{-\frac{N-1}{2}}\left(e^{-\sqrt{1-\lambda-c e^{-\theta|x|}}|x|}-e^{-2 \theta|x|}\right) \geq 0,
\end{aligned}
$$

pois dado $\varepsilon>0,1-\lambda \geq \frac{4 \varepsilon}{3}$ e $\theta=\sqrt{1-\lambda-\varepsilon}$ temos para $|x|>R, R$ suficientemente grande,

$$
\begin{aligned}
& c e^{-\theta|x|}+3(1-\lambda)-4 \varepsilon \geq 0 \\
& \Leftrightarrow 1-\lambda-c e^{-\theta|x|} \leq 4(1-\lambda-\varepsilon) \\
& \Leftrightarrow 1-\lambda-c e^{-\theta|x|} \leq 4 \theta^{2} \\
& \Leftrightarrow \sqrt{1-\lambda-c e^{-\theta|x|}} \leq 2 \theta \\
& \Leftrightarrow-\sqrt{1-\lambda-c e^{-\theta|x|}}|x| \geq-2 \theta|x| \\
& \Leftrightarrow e^{-\sqrt{1-\lambda-c e^{-\theta|x|}}|x|} \geq e^{-2 \theta|x|} \\
& \Leftrightarrow e^{-\sqrt{1-\lambda-c e^{-\theta|x|}}|x|}-e^{-2 \theta|x|} \geq 0,
\end{aligned}
$$

donde segue (2.31). Logo, de (2.29), (2.30), aplicando o Princípio do Máximo [22] obtemos

$$
H^{0}(x) \gg U(x) \text { em } \quad \mathbb{R}^{N} \backslash \bar{B}_{\rho}(0) .
$$

Portanto, combinando (2.28) e (2.32) temos

$$
H_{0}(x) \ll U(x) \ll H^{0}(x) \text { em } \mathbb{R}^{N} \backslash \bar{B}_{\rho}(0),
$$

similarmente como feito em [38]. Utilizando (2.26), (2.27) e (2.33), temos que

$$
\begin{aligned}
0<\mu_{0}=\lim _{|x| \rightarrow \infty} h_{0,1}(x)|x|^{\frac{N-1}{2}} e^{\sqrt{1-\lambda}|x|} & \leq \liminf _{|x| \rightarrow \infty} w_{1}(x)|x|^{\frac{N-1}{2}} e^{\sqrt{1-\lambda}|x|} \\
& \leq \limsup _{|x| \rightarrow \infty} w_{1}(x)|x|^{\frac{N-1}{2}} e^{\sqrt{1-\lambda}|x|} \\
& \leq \lim _{|x| \rightarrow \infty} h^{0,1}(x)|x|^{\frac{N-1}{2}} e^{\sqrt{1-\lambda}|x|}<\infty
\end{aligned}
$$


A primeira desigualdade estrita segue de (2.26) e a última desigualdade é finita, pois usando (2.27) temos

$$
\begin{aligned}
& \lim _{|x| \rightarrow \infty} h^{0,1}(x)|x|^{\frac{N-1}{2}} e^{\sqrt{1-\lambda}|x|} \\
& =\lim _{|x| \rightarrow \infty} h^{0,1}(x)|x|^{\frac{N-1}{2}} \frac{e^{\int_{\rho}^{|x|} \sqrt{1-\lambda-c e^{-\theta t}}} \mathrm{~d} t}{\int_{\rho}^{|x|} \sqrt{1-\lambda-c e^{-\theta t}} \mathrm{~d} t} e^{\sqrt{1-\lambda}|x|} \\
& e^{\lim _{|x| \rightarrow \infty} h^{0,1}(x)|x|^{\frac{N-1}{2}}} e^{\int_{\rho}^{|x|} \sqrt{1-\lambda-c e^{-\theta t}} \mathrm{~d} t} \lim _{|x| \rightarrow \infty} \frac{e^{\sqrt{1-\lambda}|x|}}{\int_{\rho}^{|x|} \sqrt{1-\lambda-c e^{-\theta t}} \mathrm{~d} t}<\infty .
\end{aligned}
$$

O próximo passo é mostrar que o $\lim _{|x| \rightarrow \infty} w_{1}(x)|x|^{\frac{N-1}{2}} e^{\sqrt{1-\lambda}|x|}$ existe. De fato, note que se mostrarmos que a função $\frac{w_{1}}{h_{0,1}}$ é não decrescente, usando (2.33) tal função seria limitada, pois $1 \leq \frac{w_{1}}{h_{0,1}} \leq \frac{h^{0,1}}{h_{0,1}} \mathrm{e}$

$$
\lim _{|x| \rightarrow \infty} \frac{h^{0,1}(x)}{h_{0,1}(x)}=\lim _{|x| \rightarrow \infty} \frac{h^{0,1}(x)|x|^{\frac{N-1}{2}} e^{\int_{\rho}^{|x|} \sqrt{1-\lambda-c e^{-\theta t}} \mathrm{~d} t}}{h_{0,1}(x)|x|^{\frac{N-1}{2}} e^{\sqrt{1-\lambda}|x|}} \frac{e^{\sqrt{1-\lambda}|x|}}{\int_{\rho}^{|x|} \sqrt{1-\lambda-c e^{-\theta t}} \mathrm{~d} t}=\frac{\mu^{0}}{\mu_{0}} C<\infty
$$

por (2.26) e (2.27). Assim concluiríamos que $\frac{w_{1}}{h_{0,1}}$ tem limite finito quando $|x| \rightarrow \infty$ e que

$$
\lim _{|x| \rightarrow \infty} w_{1}(x)|x|^{\frac{N-1}{2}} e^{\sqrt{1-\lambda}|x|}=\lim _{|x| \rightarrow \infty} \frac{w_{1}(x)}{h_{0,1}(x)} \lim _{|x| \rightarrow \infty} h_{0,1}(x)|x|^{\frac{N-1}{2}} e^{\sqrt{1-\lambda}|x|} \in(0, \infty) .
$$

Finalmente, vamos mostrar que $\frac{w_{1}}{h_{0,1}}$ é não decrescente. Considere $r, s \in(\rho, \infty)$ tal que $r \leq s$. Desde que $w_{1}$ e $h_{0,1}$ são ambas funções radiais, defina o seguinte operador

$$
S w_{1}=-\left(-\Delta w_{1}+w_{1}\right)=\Delta w_{1}-w_{1}=w_{1}^{\prime \prime}+\frac{N-1}{r} w_{1}^{\prime}-w_{1}
$$

Segue da definição do operador $S$ e de $\left(\mathcal{H}_{0}\right)$ que

$$
S h_{0,1}=h_{0,1}^{\prime \prime}+\frac{N-1}{r} h_{0,1}^{\prime}-h_{0,1}
$$

Note que, $-\Delta\left(h_{0,1}-w_{1}\right)+\left(h_{0,1}-w_{1}\right)=\lambda\left(h_{0,2}-w_{2}\right)-f\left(w_{1}, w_{2}\right)<0$, logo pelo Princípio do Máximo, temos que $h_{0,1}(x) \leq w_{1}(x)$ para todo $|x| \in(\rho, \infty)$. Agora considere a função $w=\frac{h_{0,1}}{w_{1}}$ e $\sigma(r)=\frac{N-1}{r}$. Observe que

$$
S h_{0,1}=S\left(w w_{1}\right)=w_{1} w^{\prime \prime}+\left(2 w_{1}^{\prime}+\sigma w_{1}\right) w^{\prime}+S\left(w_{1}\right) w=0
$$

Decorre que

$$
w^{\prime \prime}+\left(2 \frac{w_{1}^{\prime}}{w_{1}}+\sigma\right) w^{\prime}+\frac{1}{w_{1}} S\left(w_{1}\right) w ; \quad r \in(\rho, \infty), \quad w(\rho)=1
$$


O problema de valor inicial em (2.35) tem solução única desde que $\frac{1}{w_{1}} S\left(w_{1}\right)<0$ e $2 \frac{w_{1}^{\prime}}{w_{1}}+\sigma$ é contínua em $r \in(\rho, \infty)$.

Por outro lado, note que temos

$$
S\left(h_{0,1}-w_{1}\right)=0-\left(-f\left(w_{1}, w_{2}\right)-\lambda w_{2}\right)=f\left(w_{1}, w_{2}\right)+\lambda w_{2}>0 \text { para } r \in(\rho, \infty)
$$

e

$$
h_{0,1}-w_{1}=0 \text { sobre } \partial B_{\rho}(0)
$$

Aplicando o Princípio do Máximo e Lema de Hopf , respectivamente, temos

$$
h_{0,1}-w_{1}<0 \text { para } r>\rho \text { e } \frac{\partial\left(h_{0,1}-w_{1}\right)}{\partial r}<0 \text { se } r=\rho,
$$

que implica

$$
h_{0,1}^{\prime}(\rho)<w_{1}^{\prime}(\rho)<0
$$

Consequentemente,

$$
w^{\prime}(\rho)=\frac{h_{0,1}^{\prime}(\rho) w_{1}(\rho)-h_{0,1}(\rho) w_{1}^{\prime}(\rho)}{w_{1}^{2}(\rho)}<\frac{w_{1}^{\prime}(\rho)\left[w-1(\rho)-h_{0,1}(\rho)\right]}{w_{1}^{2}(\rho)} \leq 0 .
$$

Desde que $w(r)$ é continuamente diferenciável em $(\rho, \infty)$, derivando $w$ temos

$$
w^{\prime}(r)=\frac{h_{0,1}^{\prime}(r) w_{1}(r)-h_{0,1}(r) w_{1}^{\prime}(r)}{w_{1}^{2}(r)} .
$$

Supondo $w^{\prime}(r) \geq 0$ segue que

$$
\frac{h_{0,1}^{\prime}(r)}{h_{0,1}(r)} \geq \frac{w_{1}^{\prime}(r)}{w_{1}(r)}
$$

ou seja, $\left(\ln h_{0,1}(r)\right)^{\prime} \geq\left(\ln w_{1}(r)\right)^{\prime}$, isto é, $\ln h_{0,1}(r) \geq \ln w_{1}(r)$, Como a função $\ln (x)$ é uma função crescente, isto equivale a $h_{0,1}(r) \geq w_{1}(r)$. Contradição com (2.36). Implicando que $\frac{w_{1}}{h_{0,1}}$ é não decrescente como queríamos mostrar. Similarmente para $w_{2}$, obtendo assim

$$
\lim _{|x| \rightarrow \infty} w_{1}(x)|x|^{\frac{N-1}{2}} e^{\sqrt{1-\lambda}|x|}=\mu_{1}>0 \quad \text { e } \quad \lim _{|x| \rightarrow \infty} w_{2}(x)|x|^{\frac{N-1}{2}} e^{\sqrt{1-\lambda}|x|}=\mu_{2}>0
$$

$\operatorname{com} \mu_{1}, \mu_{2} \in(0,+\infty)$.

\subsection{Variedade de Nehari e limitação da sequência de Palais-Smale}

O resultado a seguir contém as propriedades principais sobre a variedade $\mathcal{N}$ associado ao funcional $I$.

Lema 2.4. A variedade $\mathcal{N}$ satisfaz:

a) existe um número $\alpha>0$ tal que para todo $(u, v) \in \mathcal{N}$ tem-se $\|(u, v)\| \geq \alpha$;

b) $\mathcal{N}$ é uma subvariedade fechada, de classe $C^{2}$ em $H$ e é variedade natural para I; 
c) para todo $(u, v) \in \mathcal{N}$, a função $t \mapsto I(t u, t v)$ é estritamente crescente no intervalo $[0,1)$ e estritamente decrescente no intervalo $(1,+\infty)$. Assim, em particular, pode-se afirmar que

$$
I(u, v)=\max _{t>0} I(t u, t v)>0
$$

Demonstração: Verificação de $(a)$ Por $\left(a_{1}\right),\left(F_{3}\right)$ e o funcional $J$ associado a $\mathcal{N}$ definido em $(2.4)$ tem-se

$$
\begin{aligned}
J(u, v) & =I^{\prime}(u, v)(u, v)=\|(u, v)\|^{2}-2 \lambda \int_{\mathbb{R}^{N}} u v \mathrm{~d} x-\int_{\mathbb{R}^{N}}(1+a(x)) \nabla F(u, v)(u, v) \mathrm{d} x \\
& \geq\|(u, v)\|^{2}-\lambda \int_{\mathbb{R}^{N}}\left(u^{2}+v^{2}\right) \mathrm{d} x-C \int_{\mathbb{R}^{N}} \nabla F(u, v)(u, v) \mathrm{d} x \\
& \geq(1-\lambda-\varepsilon)\|(u, v)\|^{2}-C\|(u, v)\|^{p}, \quad 2<p<2^{*} .
\end{aligned}
$$

Desde que $(u, v) \in \mathcal{N}$, tem-se $J(u, v)=0$. Assim, $(a)$ segue de

$$
C\|(u, v)\|^{p-2} \geq(1-\lambda-\varepsilon) .
$$

Verificação de (b) Note que

$$
\mathcal{N}:=\{(u, v) \in H \backslash\{(0,0)\} ; J(u, v)=0\}=J^{-1}(\{(0,0)\})
$$

Como $J$ é contínuo, implica que $\mathcal{N}$ é uma subvariedade fechada de $H^{1}\left(\mathbb{R}^{N}\right)$. Se $(u, v) \in \mathcal{N}$, por $(2.4)$ então

$$
\|(u, v)\|^{2}-2 \lambda \int_{\mathbb{R}^{N}} u v \mathrm{~d} x=\int_{\mathbb{R}^{N}}(1+a(x)) \nabla F(u, v)(u, v) \mathrm{d} x .
$$

Derivando com respeito a $u$ o gradiente $\nabla F(u, v)(u, v)=\frac{\left(u^{2}+v^{2}\right)^{2}}{1+s\left(u^{2}+v^{2}\right)}$, obtemos

$$
\begin{aligned}
& \frac{2\left(u^{2}+v^{2}\right) 2 u\left(1+s\left(u^{2}+v^{2}\right)\right) u-\left(u^{2}+v^{2}\right)^{2} 2 s u u}{\left(1+s\left(u^{2}+v^{2}\right)\right)^{2}} \\
= & \frac{4 u^{2}\left(u^{2}+v^{2}\right)+4 s u^{2}\left(u^{2}+v^{2}\right)^{2}-2 s u\left(u^{2}+v^{2}\right)^{2}}{\left(1+s\left(u^{2}+v^{2}\right)\right)^{2}} \\
= & \frac{4 u^{2}\left(u^{2}+v^{2}\right)+2 s u^{2}\left(u^{2}+v^{2}\right)^{2}}{\left(1+s\left(u^{2}+v^{2}\right)\right)^{2}}
\end{aligned}
$$

Derivando agora com respeito a $v$, analogamente, tem-se

$$
\frac{4 v^{2}\left(u^{2}+v^{2}\right)+2 s v^{2}\left(u^{2}+v^{2}\right)^{2}}{\left(1+s\left(u^{2}+v^{2}\right)\right)^{2}} .
$$

Somando (2.37) e (2.38) obtemos

$$
\frac{4\left(u^{2}+v^{2}\right)^{2}+2 s\left(u^{2}+v^{2}\right)^{3}}{\left(1+s\left(u^{2}+v^{2}\right)\right)^{2}} .
$$


Derivando (2.4) e combinado com (2.39) e o fato de que $(u, v) \in \mathcal{N}$,

$$
\begin{aligned}
J^{\prime}(u, v)(u, v)= & 2\|(u, v)\|^{2}-4 \lambda \int_{\mathbb{R}^{N}} u v \mathrm{~d} x-\int_{\mathbb{R}^{N}}(1+a(x)) \nabla^{2} F(u, v)(u, v) \mathrm{d} x \\
= & 2 \int_{\mathbb{R}^{N}}(1+a(x)) \nabla F(u, v)(u, v) \mathrm{d} x \\
& -\int_{\mathbb{R}^{N}}(1+a(x)) \frac{4\left(u^{2}+v^{2}\right)^{2}+2 s\left(u^{2}+v^{2}\right)^{3}}{\left(1+s\left(u^{2}+v^{2}\right)\right)^{2}} \mathrm{~d} x \\
= & \int_{\mathbb{R}^{N}}(1+a(x))\left\{\frac{2\left(u^{2}+v^{2}\right)^{2}}{1+s\left(u^{2}+v^{2}\right)}-\frac{4\left(u^{2}+v^{2}\right)^{2}+2 s\left(u^{2}+v^{2}\right)^{3}}{\left(1+s\left(u^{2}+v^{2}\right)\right)^{2}}\right\} \mathrm{d} x \\
= & \int_{\mathbb{R}^{N}}(1+a(x))\left\{\frac{2\left(u^{2}+v^{2}\right)^{2}+2 s\left(u^{2}+v^{2}\right)^{3}-4\left(u^{2}+v^{2}\right)^{2}-2 s\left(u^{2}+v^{2}\right)^{3}}{\left(1+s\left(u^{2}+v^{2}\right)\right)^{2}}\right\} \mathrm{d} x \\
= & \int_{\mathbb{R}^{N}}(1+a(x))\left\{\frac{-2\left(u^{2}+v^{2}\right)^{2}}{\left(1+s\left(u^{2}+v^{2}\right)\right)^{2}}\right\} \mathrm{d} x<0
\end{aligned}
$$

visto que $\inf _{x \in \mathbb{R}^{N}}(1+a(x))=\varsigma>0$, por $\left(a_{1}\right)$. Isto implica que $(0,0)$ é um valor regular de $J: H \backslash\{(0,0)\} \rightarrow$ $\mathbb{R}$. Assim, $J^{-1}(\{(0,0)\})$ é um conjunto fechado, por ser a imagem inversa de um conjunto fechado por um funcional contínuo. Como $\{(u, v) \equiv(0,0)\}$ é um ponto isolado de $J^{-1}(\{(0,0)\})$, então temos que $\mathcal{N}$ é de classe $C^{2}$ e é uma variedade natural para $I$. Assim verificamos $(b)$.

Verificação de $(c)$ Note que,

$$
\begin{aligned}
I(t u, t v) & =\frac{t^{2}}{2}\|(u, v)\|^{2}-\lambda \int_{\mathbb{R}^{N}}(t u)(t v) \mathrm{d} x-\int_{\mathbb{R}^{N}}(1+a(x)) F(t u, t v) \mathrm{d} x \\
& =\int_{\mathbb{R}^{N}}(1+a(x))\left\{\frac{t^{2}}{2} \nabla F(t u, t v)(t u, t v)-F(t u, t v)\right\} \mathrm{d} x .
\end{aligned}
$$

Considere a aplicação $\xi(t):=\frac{t^{2}}{2} \nabla F(t u, t v)(t u, t v)-F(t u, t v)$ para $t \geq 0$. Observe que

$$
\begin{aligned}
\xi(t) & =\frac{t^{2}}{2} \frac{\left(u^{2}+v^{2}\right)^{2}}{1+s\left(u^{2}+v^{2}\right)}-\frac{t^{2}\left(u^{2}+v^{2}\right)}{2 s}+\frac{1}{2 s^{2}} \ln \left(1+s t^{2}\left(u^{2}+v^{2}\right)\right) \\
& :=\frac{t^{2}}{2} \frac{z^{4}}{1+s z^{2}}-\frac{t^{2} z^{2}}{2 s}+\frac{1}{2 s^{2}} \ln \left(1+s t^{2} z^{2}\right)
\end{aligned}
$$

Derivando (2.41) em $t$, com $z^{2}=u^{2}+v^{2}$ tem-se

$$
\begin{aligned}
\xi^{\prime}(t) & =t \frac{z^{4}}{1+s z^{2}}-t \frac{z^{2}}{s}+\frac{t z^{2}}{s\left(1+s t^{2} z^{2}\right)} \\
& =\frac{t s z^{4}-t z^{2}\left(1+s z^{2}\right)}{s\left(1+s z^{2}\right)}+\frac{t z^{2}}{s\left(1+s t^{2} z^{2}\right)} \\
& =\frac{-t z^{2}}{s\left(1+s z^{2}\right)}+\frac{t z^{2}}{s\left(1+s t^{2} z^{2}\right)} \\
& =\frac{-t z^{2}-s t^{3} z^{4}+t z^{2}+s t z^{4}}{s\left(1+s z^{2}\right)\left(s t^{2} z^{2}\right)}=\frac{s t z^{2}\left(-t^{2}+1\right)}{s\left(1+s z^{2}\right)\left(s t^{2} z^{2}\right)} .
\end{aligned}
$$

Segue que se $0<t<1$, então

$$
\xi^{\prime}(t)>0
$$


Se $t>1$, então

$$
\xi^{\prime}(t)<0
$$

Se $t=1$, então

$$
\xi^{\prime}(t)=0
$$

Por $\left(a_{1}\right),(2.40),(2.42),(2.43)$ e $(2.44),(c)$ fica provado.

O próximo resultado nos dá uma limitação para uma sequência em $\mathcal{N}$ em qualquer nível $d \geq 0$ fixado. Isto nos permitirá usar o Lema de Splitting posteriormente .

Lema 2.5. Suponha que exista uma sequência $\left\{\left(u_{n}, v_{n}\right)\right\}$ em $\mathcal{N}$ satisfazendo

$$
I\left(u_{n}, v_{n}\right) \rightarrow d
$$

Então a sequência $\left\{\left(u_{n}, v_{n}\right)\right\}$ é limitada em $H$.

Demonstração: Por $\left(F_{2}\right)(b)$ temos $d \geq 0$. Fixemos $D>d \geq 0$ e consideramos que $z_{n}:=\left(u_{n}, v_{n}\right)$. Vamos supor que a sequência $\left\{z_{n}\right\}$ não seja limitada, isto é, $\left\|z_{n}\right\| \rightarrow \infty$. Considere a sequência $\left\{\varphi_{n}\right\}:=\left\{t_{n} z_{n}\right\}$ onde $t_{n}=\frac{2 \sqrt{D}}{\sqrt{1-\lambda}\left\|z_{n}\right\|}$. Assim, $\left\|\varphi_{n}\right\|=\frac{2 \sqrt{D}}{\sqrt{1-\lambda}}<\infty$, significando a limitação desta sequência. Pelo Lema 2.4, para $n$ suficientemente grande, temos que

$$
I\left(\varphi_{n}\right)=I\left(t_{n} z_{n}\right) \leq \max _{t>0} I\left(t z_{n}\right)=I\left(z_{n}\right)<D
$$

Por outro lado,

$$
\begin{aligned}
D & >I\left(t_{n} z_{n}\right)=\frac{1}{2}\left\|t_{n}\left(u_{n}, v_{n}\right)\right\|_{H}^{2}-\lambda \int_{\mathbb{R}^{N}} t_{n}^{2} u_{n} v_{n} \mathrm{~d} x-\int_{\mathbb{R}^{N}}(1+a(x)) F\left(t_{n}\left(u_{n}, v_{n}\right)\right) \mathrm{d} x \\
& \geq \frac{1}{2}\left\|t_{n}\left(u_{n}, v_{n}\right)\right\|^{2}-t_{n}^{2} \lambda \int_{\mathbb{R}^{N}}\left\{\frac{u_{n}^{2}}{2}+\frac{v_{n}^{2}}{2}\right\} \mathrm{d} x-\int_{\mathbb{R}^{N}}(1+a(x)) F\left(t_{n}\left(u_{n}, v_{n}\right)\right) \mathrm{d} x \\
& \geq \frac{1}{2}\left\|t_{n}\left(u_{n}, v_{n}\right)\right\|^{2}-t_{n}^{2} \lambda\left\|\left(u_{n}, v_{n}\right)\right\|_{H}^{2}-\int_{\mathbb{R}^{N}}(1+a(x)) F\left(t_{n}\left(u_{n}, v_{n}\right)\right) \mathrm{d} x \\
& =\left\{\frac{1-\lambda}{2}\right\}\left\|t_{n}\left(u_{n}, v_{n}\right)\right\|_{H}^{2}-\int_{\mathbb{R}^{N}}(1+a(x)) F\left(t_{n}\left(u_{n}, v_{n}\right)\right) \mathrm{d} x \\
& =\left\{\frac{1-\lambda}{2}\right\} \frac{4 D}{1-\lambda}-\int_{\mathbb{R}^{N}}(1+a(x)) F\left(t_{n}\left(u_{n}, v_{n}\right)\right) \mathrm{d} x .
\end{aligned}
$$

Isto implica que

$$
\int_{\mathbb{R}^{N}}(1+a(x)) F\left(t_{n}\left(u_{n}, v_{n}\right)\right) \mathrm{d} x \geq 2 D-D=D .
$$

Para obtermos a contradição, precisamos mostrar que $\int_{\mathbb{R}^{N}}(1+a(x)) F\left(t_{n}\left(u_{n}, v_{n}\right)\right) \mathrm{d} x=o_{n}(1)$, pois $D>0$. De fato, desde que $\left\{\varphi_{n}\right\}$ é limitada em $H$, então $\varphi_{n} \rightarrow \varphi$ em $H$. Temos dois casos a considerar:

1. $\lim _{n \rightarrow+\infty}\left(\sup _{y \in \mathbb{R}^{N}} \int_{B_{1}(y)}\left|\varphi_{n}\right|^{2} \mathrm{~d} x\right)=0 ;$ 
2. $\lim _{n \rightarrow+\infty}\left(\sup _{y \in \mathbb{R}^{N}} \int_{B_{1}(y)}\left|\varphi_{n}\right|^{2} \mathrm{~d} x\right)=\eta>0$.

Suponha que o primeiro caso seja verdade. Usando o Lema de Lions, logo $\varphi_{n} \rightarrow 0$ em $L^{p}\left(\mathbb{R}^{N}\right)$ para $2<p<2^{*}$. Usando $\left(F_{3}\right)$, como $\varepsilon>0$ é arbitrário, $\varphi_{n}$ é limitada e $\left\|\varphi_{n}\right\|_{L^{p}} \rightarrow 0$, segue que

$$
\int_{\mathbb{R}^{N}}(1+a(x)) F\left(t_{n}\left(u_{n}, v_{n}\right)\right) \mathrm{d} x \leq C\left(\varepsilon\left\|\varphi_{n}\right\|_{L^{2}}^{2}+C_{\varepsilon}\left\|\varphi_{n}\right\|_{L^{p}}^{p}\right)<D .
$$

Contradição. Então suponha que o segundo caso ocorra, a menos de subsequência, fixando $n \in \mathbb{N}$, por definição de supremo existe uma sequência $\left\{y_{n}\right\} \subset \mathbb{R}^{N}$ tal que

$$
\int_{B_{1}\left(y_{n}\right)}\left|\varphi_{n}^{1}\right|^{2} \mathrm{~d} x \geq \frac{\eta}{4}
$$

onde $\varphi_{n}=\left(\varphi_{n}^{1}, \varphi_{n}^{2}\right)$. Defina $\varphi_{n}^{1}:=\varphi_{n}^{1}\left(x+y_{n}\right)$. Desde que $\varphi_{n}$ é limitada e pela invariância do domínio, $\varphi_{n}^{1}$ é limitada em $H^{1}$ e, portanto, converge fraco para alguma $\varphi^{1}$. Temos ainda que $\varphi_{n}^{1} \rightarrow \varphi^{1}$ em $L_{l o c}^{p}\left(\mathbb{R}^{N}\right)$ para $2 \leq p<2^{*}$ e também q.t.p. em $\mathbb{R}^{N}$.

Usando mudanca de variáveis, segue que

$$
\begin{aligned}
\int_{B_{1}(0)}\left|\varphi^{1}(x)\right|^{2} \mathrm{~d} x & =\lim _{n \rightarrow+\infty} \int_{B_{1}(0)}\left|\varphi_{n}^{1}\left(x+y_{n}\right)\right|^{2} \mathrm{~d} x \\
& =\lim _{n \rightarrow+\infty} \int_{B_{1}\left(y_{n}\right)}\left|\varphi_{n}^{1}(x)\right|^{2} \mathrm{~d} x \geq \frac{\eta}{4}>0 .
\end{aligned}
$$

Assim existe um subconjunto $\Omega \subset \mathbb{R}^{N}$ de medida de Lebesgue positiva em $B_{1}(0)$ em que $\varphi(x) \not \equiv$ $0, \forall x \in \Omega$. Observe que a convergência pontual em $\mathbb{R}^{N}$ nos dá

$$
0<\left|\varphi^{1}(x)\right|=\lim _{n \rightarrow+\infty}\left|\varphi_{n}^{1}\left(x+y_{n}\right)\right|=\lim _{n \rightarrow+\infty} \frac{2 \sqrt{D}\left|u_{n}\left(x+y_{n}\right)\right|}{\left\|z_{n}\left(x+y_{n}\right)\right\|}=\lim _{n \rightarrow+\infty} \frac{2 \sqrt{D}\left|u_{n}\left(x+y_{n}\right)\right|}{\left\|z_{n}\right\|},
$$

para todo $x \in \Omega$. Visto que $\left\|z_{n}\right\| \rightarrow+\infty$, quando $n \rightarrow+\infty$, necessariamente temos que

$$
\left|u_{n}\left(x+y_{n}\right)\right| \rightarrow+\infty, \text { para todo } x \in \Omega
$$

Desde que $\left\{\left(u_{n}, v_{n}\right)\right\}$ está contida em $\mathcal{N}$, podemos usar o Lema de Fatou, a condição de não quadrati- 
cidade $\left(F_{2}\right),\left(a_{1}\right)$ e obter

$$
\begin{aligned}
D> & \lim _{n \rightarrow+\infty} I\left(u_{n}, v_{n}\right)=\lim _{n \rightarrow+\infty}\left(\frac{1}{2}\left\|\left(u_{n}, v_{n}\right)\right\|_{H^{1}\left(\mathbb{R}^{N}\right)}^{2}-\lambda \int_{\mathbb{R}^{N}} u_{n} v_{n} \mathrm{~d} x\right) \\
& -\lim _{n \rightarrow+\infty}\left(\int_{\mathbb{R}^{N}}(1+a(x)) F\left(u_{n}, v_{n}\right) \mathrm{d} x\right) \\
= & \lim _{n \rightarrow+\infty} \int_{\mathbb{R}^{N}}(1+a(x))\left(\frac{1}{2} \nabla F\left(u_{n}, v_{n}\right)\left(u_{n}, v_{n}\right)-F\left(u_{n}, v_{n}\right)\right) \mathrm{d} x \\
\geq & \liminf _{n \rightarrow+\infty} \int_{\mathbb{R}^{N}} \varsigma\left(\frac{1}{2} \nabla F\left(u_{n}, v_{n}\right)\left(u_{n}, v_{n}\right)-F\left(u_{n}, v_{n}\right)\right) \mathrm{d} x \\
= & \liminf _{n \rightarrow+\infty} \int_{\mathbb{R}^{N}}\left(\varsigma \frac{1}{2} \nabla F\left(u_{n}\left(x+y_{n}\right), v_{n}\left(x+y_{n}\right)\right)\left(u_{n}\left(x+y_{n}\right), v_{n}\left(x+y_{n}\right)\right)\right. \\
& -\varsigma F\left(u_{n}\left(x+y_{n}\right), v_{n}\left(x+y_{n}\right)\right) \mathrm{d} x \\
> & \liminf _{n \rightarrow+\infty} \int_{\Omega}\left(\varsigma \frac{1}{2} \nabla F\left(u_{n}\left(x+y_{n}\right), v_{n}\left(x+y_{n}\right)\right)\left(u_{n}\left(x+y_{n}\right), v_{n}\left(x+y_{n}\right)\right)\right. \\
& \left.-\varsigma F\left(u_{n}\left(x+y_{n}\right), v_{n}\left(x+y_{n}\right)\right)\right) \mathrm{d} x \\
\geq & \varsigma \int_{\Omega}\left(\liminf _{n \rightarrow+\infty} \frac{1}{2} \nabla F\left(u_{n}\left(x+y_{n}\right), v_{n}\left(x+y_{n}\right)\right)\left(u_{n}\left(x+y_{n}\right), v_{n}\left(x+y_{n}\right)\right)\right. \\
& \left.-F\left(u_{n}\left(x+y_{n}\right), v_{n}\left(x+y_{n}\right)\right)\right) \mathrm{d} x=+\infty .
\end{aligned}
$$

Assim, o segundo caso também não pode ocorrer. A contradição veio do fato de supor que a sequência $\left\{\left(u_{n}, v_{n}\right)\right\}$ não era limitada em $H$ e obtemos assim o resultado desejado.

O resultado a seguir mostra a positividade do ínfimo do funcional associado ao problema $(\mathbb{S})$ sobre a variedade $\mathcal{N}$.

Lema 2.6. Seja $m$ definida em (2.5), então tem-se $m>0$.

Demonstração: A demonstração é análoga à do Lema 2.5. Considere $\left\{\left(u_{n}, v_{n}\right)\right\}$ na variedade $\mathcal{N}$ tal que $I\left(u_{n}, v_{n}\right) \rightarrow m$. Pelo Lema 2.5, $\left\{\left(u_{n}, v_{n}\right)\right\}$ é limitada em $H$ e $\left(u_{n}, v_{n}\right) \rightarrow(u, v)$ com $u \not \equiv 0$ e $v \not \equiv 0$. Segue que

$$
\begin{aligned}
m= & \lim _{n \rightarrow+\infty} I\left(u_{n}, v_{n}\right)=\lim _{n \rightarrow+\infty}\left(\frac{1}{2}\left\|\left(u_{n}, v_{n}\right)\right\|^{2}-\lambda \int_{\mathbb{R}^{N}} u_{n} v_{n} \mathrm{~d} x\right) \\
& -\lim _{n \rightarrow+\infty}\left(\int_{\mathbb{R}^{N}}(1+a(x)) F\left(u_{n}, v_{n}\right) \mathrm{d} x\right) \\
\geq & \varsigma \int_{\Omega}\left(\liminf _{n \rightarrow+\infty} \frac{1}{2} \nabla F\left(u_{n}\left(x+y_{n}\right), v_{n}\left(x+y_{n}\right)\right)\left(u_{n}\left(x+y_{n}\right), v_{n}\left(x+y_{n}\right)\right)\right. \\
& \left.-F\left(u_{n}\left(x+y_{n}\right), v_{n}\left(x+y_{n}\right)\right)\right) \mathrm{d} x \\
= & \varsigma \int_{\Omega}\left(\frac{1}{2} \nabla F(u, v)(u, v)-F(u, v)\right) \mathrm{d} x>0 .
\end{aligned}
$$


Observação 2.2. Note que o resultado obtido no Lema 2.6 também se aplica ao funcional $I_{\infty}$, ou seja, $m_{\infty}>0$.

O próximo lema nos dá uma importante informação sobre uma sequência $(P S)$ do funcional $I$ restrito à $\mathcal{N}$ e no espaço $H$.

Lema 2.7. Suponha que $\left\{\left(u_{n}, v_{n}\right)\right\}$ é uma sequência $(P S)_{d}$ para I restrito à variedade $\mathcal{N}$. Então, a menos de subsequência, a sequência $\left\{\left(u_{n}, v_{n}\right)\right\}$ é também uma sequência $(P S)_{d}$ para o funcional I em $H^{1}\left(\mathbb{R}^{N}\right) \times H^{1}\left(\mathbb{R}^{N}\right)$.

Demonstração: Considere $\left\{\left(u_{n}, v_{n}\right)\right\} \subset \mathcal{N}$ do tipo $(P S)_{d}$. Então pelo Lema $2.5,\left\{\left(u_{n}, v_{n}\right)\right\}$ é limitada e satisfaz

$$
I\left(u_{n}, v_{n}\right) \rightarrow d \quad \text { e } \quad\left(\left.I\right|_{\mathcal{N}}\right)^{\prime}\left(u_{n}, v_{n}\right) \rightarrow 0 .
$$

Observe que para qualquer $(\varphi, \psi) \in H$

$$
\begin{aligned}
J^{\prime}\left(u_{n}, v_{n}\right)(\varphi, \psi)= & 2\left(\left\langle u_{n}, \varphi\right\rangle+\left\langle v_{n}, \psi\right\rangle\right)-2 \lambda \int_{\mathbb{R}^{N}}\left(v_{n} \varphi+u_{n} \psi\right) \mathrm{d} x \\
& -\int_{\mathbb{R}^{N}}(1+a(x)) \nabla\left(\nabla F\left(u_{n}, v_{n}\right)\left(u_{n}, v_{n}\right)\right)(\varphi, \psi) \mathrm{d} x .
\end{aligned}
$$

Usando a hipótese $\left(a_{1}\right)$ e a desigualdade de Hölder segue que

$$
\begin{aligned}
\left|J^{\prime}\left(u_{n}, v_{n}\right)(\varphi, \psi)\right| \leq & 2\left\{\left\|u_{n}\right\|\|\varphi\|+\left\|v_{n}\right\|\|\psi\|\right\}+2 \int_{\mathbb{R}^{N}}\left(\left|v_{n}\left\|\varphi|+| u_{n}\right\| \psi\right|\right) \mathrm{d} x \\
& +C \int_{\mathbb{R}^{N}}\left|\nabla\left(\nabla F\left(u_{n}, v_{n}\right)\left(u_{n}, v_{n}\right)\right)(\varphi, \psi)\right| \mathrm{d} x \\
\leq & 2\left\{\left\|u_{n}\right\|\|\varphi\|+\left\|v_{n}\right\|\|\psi\|\right\} \\
& +2\left\{\left(\int_{\mathbb{R}^{N}}\left|v_{n}\right|^{2} \mathrm{~d} x\right)^{1 / 2}\left(\int_{\mathbb{R}^{N}}|\varphi|^{2} \mathrm{~d} x\right)^{1 / 2}+\left(\int_{\mathbb{R}^{N}}\left|u_{n}\right|^{2} \mathrm{~d} x\right)^{1 / 2}\left(\int_{\mathbb{R}^{N}}|\psi|^{2} \mathrm{~d} x\right)^{1 / 2}\right\} \\
& +C \int_{\mathbb{R}^{N}}\left|\nabla\left(\nabla F\left(u_{n}, v_{n}\right)\left(u_{n}, v_{n}\right)\right)(\varphi, \psi)\right| \mathrm{d} x \\
\leq & 2\left\{\left\|u_{n}\right\|\|\varphi\|+\left\|v_{n}\right\|\|\psi\|\right\}+2\left\{\left\|v_{n}\right\|\|\varphi\|+\left\|u_{n}\right\|\|\psi\|\right\} \\
& +C \int_{\mathbb{R}^{N}} \frac{4\left|u_{n}\right|\left(u_{n}^{2}+v_{n}^{2}\right)+2 s\left|u_{n}\right|\left(u_{n}^{2}+v_{n}^{2}\right)^{2}}{\left(1+s\left(u_{n}^{2}+v_{n}^{2}\right)\right)^{2}} \\
& +C \int_{\mathbb{R}^{N}} \frac{4\left|v_{n}\right|\left(u_{n}^{2}+v_{n}^{2}\right)+2 s\left|v_{n}\right|\left(u_{n}^{2}+v_{n}^{2}\right)^{2}}{\left(1+s\left(u_{n}^{2}+v_{n}^{2}\right)\right)^{2}} \\
& \left.2\left\{\left\|u_{n}\right\|+\left\|v_{n}\right\|\right\}\{\|\varphi\|+\|\psi\|\}+C \iint_{\mathbb{R}^{N}} \frac{4\left|u_{n} \| \varphi\right|}{s}+\frac{4\left|v_{n} \| \psi\right|}{s}\right\} \mathrm{d} x
\end{aligned}
$$




$$
\begin{aligned}
& \leq\left\|\left(u_{n}, v_{n}\right)\right\|\|(\varphi, \psi)\|+\frac{4 C}{s}\left\{\left\|v_{n}\right\|\|\varphi\|+\left\|u_{n}\right\|\|\psi\|\right\} \\
& \leq 2\left\|\left(u_{n}, v_{n}\right)\right\|\|(\varphi, \psi)\|+\frac{4 C}{s}\left\{\left(\left\|v_{n}\right\|+\| u_{n}\right)\|\varphi\|+\left(\left\|u_{n}\right\|+\| v_{n}\right)\|\psi\|\right\} \\
& \leq\left\|\left(u_{n}, v_{n}\right)\right\|\|(\varphi, \psi)\|+\frac{4 C}{s}\left\|\left(u_{n}, v_{n}\right)\right\|\|(\varphi, \psi)\| \\
& \leq \max \left\{2, \frac{4 C}{s}\right\}\left\|\left(u_{n}, v_{n}\right)\right\|\|(\varphi, \psi)\| .
\end{aligned}
$$

Desde que a sequência $\left\{\left(u_{n}, v_{n}\right)\right\}$ é limitada em $H$, então para toda $(\varphi, \psi) \in H$,

$$
\left|J^{\prime}\left(u_{n}, v_{n}\right)(\varphi, \psi)\right| \leq C\|(\varphi, \psi)\|_{H}
$$

Isto mostra que a sequência $\left\{J^{\prime}\left(u_{n}, v_{n}\right)\right\}$ é limitada em $H^{-1}$. Consequentemente, $\left|J^{\prime}\left(u_{n}, v_{n}\right)\left(u_{n}, v_{n}\right)\right| \leq$ $\left\|J^{\prime}\left(u_{n}, v_{n}\right)\right\|_{H^{-1}}\left\|\left(u_{n}, v_{n}\right)\right\|_{H} \leq C$, onde a constante $C$ não depende de $\left\{\left(u_{n}, v_{n}\right)\right\}$. A menos de subsequência, a sequência de números reais positivos

$$
\varrho_{n}:=\left|J^{\prime}\left(u_{n}, v_{n}\right)\left(u_{n}, v_{n}\right)\right| \rightarrow \varrho \geq 0 .
$$

Por outro lado, note que

$$
\begin{aligned}
\varrho & =\liminf _{n \rightarrow \infty}\left|J^{\prime}\left(u_{n}, v_{n}\right)\left(u_{n}, v_{n}\right)\right| \\
& =\liminf _{n \rightarrow \infty}\left|2\left\|\left(u_{n}, v_{n}\right)\right\|^{2}-4 \lambda \int_{\mathbb{R}^{N}} u_{n} v_{n} \mathrm{~d} x-\int_{\mathbb{R}^{N}}(1+a(x)) \nabla\left(\nabla F\left(u_{n}, v_{n}\right)\left(u_{n}, v_{n}\right)\right)\left(u_{n}, v_{n}\right) \mathrm{d} x\right| \\
& =\liminf _{n \rightarrow \infty}\left|\int_{\mathbb{R}^{N}}(1+a(x)) 2 \nabla F\left(u_{n}, v_{n}\right)\left(u_{n}, v_{n}\right) \mathrm{d} x-\int_{\mathbb{R}^{N}}(1+a(x)) \nabla\left(\nabla F\left(u_{n}, v_{n}\right)\left(u_{n}, v_{n}\right)\right)\left(u_{n}, v_{n}\right) \mathrm{d} x\right| \\
& =\liminf _{n \rightarrow \infty}\left|\int_{\mathbb{R}^{N}}(1+a(x))\left\{\frac{2\left(u_{n}^{2}+v_{n}^{2}\right)^{2}}{\left(1+s\left(u_{n}^{2}+v_{n}^{2}\right)\right)^{2}}\right\} \mathrm{d} x\right| \\
& \geq \varsigma \liminf _{n \rightarrow \infty} \int_{\mathbb{R}^{N}}\left\{\frac{2\left(u_{n}^{2}+v_{n}^{2}\right)^{2}}{\left(1+s\left(u_{n}^{2}+v_{n}^{2}\right)\right)^{2}}\right\} \mathrm{d} x \\
& \geq \varsigma \liminf _{n \rightarrow \infty} \int_{\Omega_{0}}\left\{\frac{2\left(u_{n}^{2}+v_{n}^{2}\right)^{2}}{\left(1+s\left(u_{n}^{2}+v_{n}^{2}\right)\right)^{2}}\right\} \mathrm{d} x \\
& =\varsigma \int_{\Omega_{0}}\left\{\frac{2\left(u^{2}+v^{2}\right)^{2}}{\left(1+s\left(u^{2}+v^{2}\right)\right)^{2}}\right\} \mathrm{d} x>0,
\end{aligned}
$$

pois como $\left\{\left(u_{n}, v_{n}\right)\right\} \in \mathcal{N}$, pelo Lema 2.4 temos que $\left\|\left(u_{n}, v_{n}\right)\right\| \geq \alpha>0$ e argumentando como na demonstração do Lema 2.6, usando o Lema de Lions tem-se que $\left(u_{n}(x), v_{n}(x)\right) \rightarrow(u(x), v(x))$ q.t.p. $x \in \mathbb{R}^{N}$ e $u(x), v(x) \neq 0$ q.t.p. $x \in \Omega_{0}$ onde $\Omega_{0}$ é um conjunto de medida positiva, e tudo isto adicionado com o Lema de Fatou e $\left(a_{1}\right)$.

Como $\mathcal{N}$ é uma variedade de codimensão 1 em $H$ (vide seção 6.3 em [7]) podemos escrever a projeção gradiente $\left(\left.I\right|_{\mathcal{N}}\right)^{\prime}(u, v)$ sobre o plano tangente

$$
T_{(u, v)} \mathcal{N}=\left\{(\varphi, \psi) \in H:\left\langle J^{\prime}(u),(\varphi, \psi)\right\rangle=0\right\}
$$


por

$$
\left(\left.I\right|_{\mathcal{N}}\right)^{\prime}(u, v)=I^{\prime}(u, v)-t J^{\prime}(u, v)
$$

em que

$$
t:=\frac{\left\langle I^{\prime}(u, v), J^{\prime}(u, v)\right\rangle}{\left\|J^{\prime}(u, v)\right\|^{2}} .
$$

Desde que $\left\{\left(u_{n}, v_{n}\right)\right\} \subset \mathcal{N}$ é uma sequência $(P S)$ de $I$ restrito a $\mathcal{N}$ (vide Lema 7.19 em [7]) existe uma sequência $\left\{t_{n}\right\}$ tal que

$$
\left(\left.I\right|_{\mathcal{N}}\right)^{\prime}\left(u_{n}, v_{n}\right)=I^{\prime}\left(u_{n}, v_{n}\right)-t_{n} J^{\prime}\left(u_{n}, v_{n}\right)=o_{n}(1),
$$

dada por

$$
t_{n}:=\frac{\left\langle I^{\prime}\left(u_{n}, v_{n}\right), J^{\prime}\left(u_{n}, v_{n}\right)\right\rangle}{\left\|J^{\prime}\left(u_{n}, v_{n}\right)\right\|^{2}} ;
$$

note que $\left\|J^{\prime}\left(u_{n}, v_{n}\right)\right\| \neq 0$ pelo Lema 2.4.

O objetivo agora é mostrar que a sequência $\left\{t_{n}\right\}$ converge a zero, se $n \rightarrow+\infty$. Assim concluíremos o lema.

Por (2.48) e (2.49) segue que

$$
\begin{aligned}
0 & =I^{\prime}\left(u_{n}, v_{n}\right)\left(u_{n}, v_{n}\right) \\
& =\left(\left.I\right|_{\mathcal{N}}\right)^{\prime}\left(u_{n}, v_{n}\right)\left(u_{n}, v_{n}\right)+t_{n} J^{\prime}\left(u_{n}, v_{n}\right)\left(u_{n}, v_{n}\right) \\
& =o_{n}(1)+t_{n} J^{\prime}\left(u_{n}, v_{n}\right)\left(u_{n}, v_{n}\right) .
\end{aligned}
$$

Por (2.49) e (2.50) tem-se $C \geq\left|J^{\prime}\left(u_{n}, v_{n}\right)\left(u_{n}, v_{n}\right)\right| \rightarrow \varrho>0$, se $n \rightarrow+\infty$, e isto implica que, a menos de subsequência,

$$
t_{n} \rightarrow 0, \text { se } n \rightarrow+\infty .
$$

Assim, deduzimos de (2.48) e (2.51) que, a menos de subsequência de $\left\{\left(u_{n}, v_{n}\right)\right\}$,

$$
I^{\prime}\left(u_{n}, v_{n}\right)=\left(\left.I\right|_{\mathcal{N}}\right)^{\prime}\left(u_{n}, v_{n}\right)+t_{n} J^{\prime}\left(u_{n}, v_{n}\right) \rightarrow 0, \text { se } \quad n \rightarrow+\infty .
$$

Concluimos assim a demonstração do lema.

Pretendemos usar ferramentas do tipo min-max para encontrar solução de (S). Entretanto, o conjunto de soluções do sistema autônomo pode ser complicado e, ao contrário do caso escalar, precisamos impor hipóteses similares às que implicam em unicidade de solução no caso escalar. Definimos

$$
\widehat{m}_{\infty}=\inf \left\{m^{*}>m_{\infty}: m^{*} \text { é valor crítico de } I_{\infty}\right\} .
$$

Observação 2.3. O problema escalar que tem solução positiva única satisfaz esta hipótese. Supor que o sistema tenha solução positiva $(u, v)$ única é mais artificial, entretanto podemos supor que todas as soluções positivas (caso exista mais de uma) estejam no mesmo nivel de energia é razoável. 
Observação 2.4. Em 2013 Maia-Montefusco-Pellacci [32] investigaram o problema fracamente acoplado

$$
\left\{\begin{array}{cl}
-\Delta u+\lambda u=\frac{u\left(u^{2}+v^{2}\right)}{1+s\left(u^{2}+v^{2}\right)}, & \mathbb{R}^{N}, \\
-\Delta v+\lambda v=\frac{v\left(u^{2}+v^{2}\right)}{1+s\left(u^{2}+v^{2}\right)}, & \mathbb{R}^{N}, \\
u, v \in H^{1}\left(\mathbb{R}^{N}\right), &
\end{array}\right.
$$

com $\lambda>0$ e $N \geq 2$. O Teorema 2.1 em [32] mostra que se $s \in(0,1 / \lambda)$, então existe uma única solução $U=(u, v)$, a menos de rotação, com ambas componentes positivas de $(2.52)$ dada por $(u, v)=$ $(\cos \theta, \sin \theta) z_{\lambda}$ onde $\theta \in(0, \pi / 2)$ e $z_{\lambda}$ é solução de

$$
\left\{\begin{array}{l}
-\Delta z_{\lambda}+\lambda z_{\lambda}=\frac{z_{\lambda}^{3}}{1+s z_{\lambda}^{2}}, \quad \mathbb{R}^{N} \\
z_{\lambda} \in H^{1}\left(\mathbb{R}^{N}\right)
\end{array}\right.
$$

Outrossim, o nível de energia da única solução vetorial do sistema (2.52) é igual ao nível de energia das soluções de (2.53). Em outras palavras, para o problema modelo (2.52) não podemos encontrar uma solução vetorial com nivel de energia estritamente menor que o nivel de energia das soluções de (2.53).

Assumindo a hipótese $(U)$ vamos mostrar que $2 m_{\infty} \leq \widehat{m}_{\infty}$. Precisaremos do seguinte lema auxiliar

Lema 2.8. Se $(u, v)$ é solução de $\left(\mathbb{S}_{\infty}\right)$ que muda de sinal, então

$$
\operatorname{supt}\left(u^{+}\right) \cap \operatorname{supt}\left(v^{-}\right) \subset\{x: u(x)=0 \text { e } v(x)=0\}
$$

onde $u^{+}=\max \{u, 0\}$ e $v^{-}=\max \{-v, 0\}$.

Demonstração: Primeiramente note que o acoplamento forte do sistema $\left(\mathbb{S}_{\infty}\right)$ implica $v=0$ se, e somente se, $u=0$ (isto é, as regiões nodais de $u$ e $v$ são as mesmas). Considere $\Omega:=\operatorname{supt}\left\{u^{+}\right\} \subset \mathbb{R}^{N}$. Se $v(x)<0$ para algum $x \in \Omega$, então $x \in \operatorname{int}(\Omega)$, (pois $u(x)=0$ para todo $x \in \partial \Omega$ ). Logo, obtemos que $\Omega:=\operatorname{supt}\left\{v^{-}\right\} \subset \mathbb{R}^{N}$. Dado $x_{0} \in \partial \Omega, u\left(x_{0}\right)=v\left(x_{0}\right)=0$ e pela continuidade de $u$ e $v$ dado um $\varepsilon>0$ existe um $\delta>0$ tal que, se $x \in \Omega$ e $\left|x-x_{0}\right|<\delta$, então $\left|u^{+}(x)\right|=|u(x)|<\varepsilon$ e $\left|v^{-}(x)\right|=|v(x)|<\varepsilon$. Subtraindo as duas equações do sistema $\left(\mathbb{S}_{\infty}\right)$, temos

$$
-\Delta(u-v)+(u-v)=\frac{u^{2}+v^{2}}{1+s\left(u^{2}+v^{2}\right)}(u-v)-\lambda(u-v)
$$

e para todos os pontos $x \in \Omega$, vamos ter

$$
-\Delta\left(u^{+}+v^{-}\right)+\left[1+\lambda-\frac{u^{2}+v^{2}}{1+s\left(u^{2}+v^{2}\right)}\right]\left(u^{+}+v^{-}\right)=0
$$

Tomando $\varepsilon$ suficientemente pequeno tal que $\frac{u^{2}+v^{2}}{1+s\left(u^{2}+v^{2}\right)}<\frac{1+\lambda}{2}$, para todo $x \in \Omega$ e $\left|x-x_{0}\right|<\delta$. 
Multiplicando a equação (2.55) por $u^{+}+v^{-}$obtemos

$$
\begin{aligned}
0 & =\int_{\Omega \cap B_{\delta}\left(x_{0}\right)}\left(\left|\nabla\left(u^{+}+v^{-}\right)\right|^{2}+\left[1+\lambda-\frac{u^{2}+v^{2}}{1+s\left(u^{2}+v^{2}\right)}\right]\left(u^{+}+v^{-}\right)^{2}\right) \mathrm{d} x \\
& >\int_{\Omega \cap B_{\delta}\left(x_{0}\right)}\left(\left|\nabla\left(u^{+}+v^{-}\right)\right|^{2}+\left[\frac{1+\lambda}{2}\right]\left(u^{+}+v^{-}\right)^{2}\right) \mathrm{d} x>0
\end{aligned}
$$

visto que, $u^{+}(x)+v^{-}(x)>0$ no interior $\Omega \cap B_{\delta}\left(x_{0}\right)$, pelo Lema de Hopf e do fato que $u$ e $v$ são soluções clássicas de $\left(\mathbb{S}_{\infty}\right)$. Este absurdo veio de termos assumido que $v(x)<0$ para algum $x \in \Omega$, portanto $v(x) \geq 0$ para todo $x \in \Omega$ e $\operatorname{assim} \operatorname{supt}\left(u^{+}\right) \cap \operatorname{supt}\left(v^{-}\right) \subset\{x: u(x)=0$ e $v(x)=0\}$. Analogamente verifica-se que

$$
\operatorname{supt}\left(u^{-}\right) \cap \operatorname{supt}\left(v^{+}\right) \subset\{x: u(x)=0 \text { e } v(x)=0\} .
$$

Segue o seguinte lema de não existência de solução

Lema 2.9. Suponha $(U)$, então não existe solução $(u, v)$ para o problema $\left(\mathbb{S}_{\infty}\right)$ tal que $I_{\infty}(u, v) \in$ $\left(m_{\infty}, 2 m_{\infty}\right)$

Demonstração: Seja $(u, v)$ uma solução de $\left(\mathbb{S}_{\infty}\right)$. Segue que $I_{\infty}^{\prime}(u, v)=0$, isto é, $I_{\infty}^{\prime}(u, v)(\phi, \psi)=0$ para qualquer $(\phi, \psi) \in H$. Em particular, $I_{\infty}^{\prime}(u, v)\left(u^{+}, v^{+}\right)=0$ e $I_{\infty}^{\prime}(u, v)\left(u^{-}, v^{-}\right)=0$. Desejamos mostrar que $\left(u^{+}, v^{+}\right)$e $\left(u^{-}, v^{-}\right)$estão na variedade $\mathcal{N}$. De fato,

$$
\begin{aligned}
0= & I_{\infty}^{\prime}(u, v)\left(u^{+}, v^{+}\right) \\
= & \int_{\mathbb{R}^{N}}\left[\nabla\left(u^{+}+u^{-}\right) \nabla u^{+}+\nabla\left(v^{+}+v^{-}\right) \nabla v^{+}+\left(u^{+}+u^{-}\right) u^{+}+\left(v^{+}+v^{-}\right) v^{-}\right] \mathrm{d} x \\
& -\lambda \int_{\mathbb{R}^{N}}\left[\left(v^{+}+v^{-}\right) u^{+}+\left(u^{+}+u^{-}\right) v^{+}\right] \mathrm{d} x-\int_{\mathbb{R}^{N}} \nabla F\left(u^{+}+u^{-}, v^{+}+v^{-}\right)\left(u^{+}, v^{+}\right) \mathrm{d} x \\
= & \int_{\mathbb{R}^{N}}\left[\left|\nabla u^{+}\right|^{2}+\left|\nabla v^{+}\right|^{2}+\left(u^{+}\right)^{2}+\left(v^{+}\right)^{2}\right] \mathrm{d} x-\lambda \int_{\mathbb{R}^{N}}\left(u^{+} v^{+}+u^{+} v^{+}+v^{-} u^{+}+u^{-} v^{+}\right) \mathrm{d} x \\
& -\int_{\mathbb{R}^{N}} \nabla F\left(u^{+}+u^{-}, v^{+}+v^{-}\right)\left(u^{+}, v^{+}\right) \mathrm{d} x \\
= & \int_{\mathbb{R}^{N}}\left[\left|\nabla u^{+}\right|^{2}+\left|\nabla v^{+}\right|^{2}+\left(u^{+}\right)^{2}+\left(v^{+}\right)^{2}\right] \mathrm{d} x-\lambda \int_{\mathbb{R}^{N}}\left(u^{+} v^{+}+u^{+} v^{+}+v^{-} u^{+}+u^{-} v^{+}\right) \mathrm{d} x \\
& -\int_{\{u, v \geq 0\}} \nabla F\left(u^{+}, v^{+}\right)\left(u^{+}, v^{+}\right) \mathrm{d} x-\int_{\{u, v<0\}} \nabla F\left(u^{-}, v^{-}\right)\left(u^{+}, v^{+}\right) \mathrm{d} x \\
& -\int_{\{u \geq 0, v \leq 0\}} \nabla F\left(u^{+}, v^{-}\right)\left(u^{+}, v^{+}\right) \mathrm{d} x-\int_{\{u<0, v>0\}} \nabla F\left(u^{-}, v^{+}\right)\left(u^{+}, v^{+}\right) \mathrm{d} x \\
= & I_{\infty}^{\prime}\left(u^{+}, v^{+}\right)\left(u^{+}, v^{+}\right),
\end{aligned}
$$

em que a última desigualdade é consequência de $(2.54)$ e $(2.56)$. Logo, $\left(u^{+}, v^{+}\right) \in \mathcal{N}_{\infty}$. De modo análogo mostra-se que $\left(u^{-}, v^{-}\right) \in \mathcal{N}_{\infty}$. 
Agora, note que

$$
\begin{aligned}
& I_{\infty}\left(u^{+}+u^{-}, v^{+}+v^{-}\right) \\
= & \frac{1}{2}\left\|\left(u^{+}+u^{-}, v^{+}+v^{-}\right)\right\|^{2}-\lambda \int_{\mathbb{R}^{N}}\left(u^{+}+u^{-}, v^{+}+v^{-}\right)\left(u^{+}+u^{-}, v^{+}+v^{-}\right) \mathrm{d} x \\
& -\int_{\mathbb{R}^{N}} F\left(u^{+}+u^{-}, v^{+}+v^{-}\right) \mathrm{d} x \\
= & \frac{1}{2}\left\|\left(u^{+}, v^{+}\right)\right\|^{2}+\frac{1}{2}\left\|\left(u^{-}, v^{-}\right)\right\|^{2}-\lambda \int_{\mathbb{R}^{N}}\left(u^{+} v^{+}+u^{-} v^{-}\right) \mathrm{d} x \\
& -\int_{\{u, v \geq 0\}} F\left(u^{+}, v^{+}\right) \mathrm{d} x-\int_{\{u, v<0\}} F\left(u^{-}, v^{-}\right) \mathrm{d} x \\
& -\int_{\{u \geq 0, v \leq 0\}} F\left(u^{+}, v^{-}\right) \mathrm{d} x-\int_{\{u<0, v>0\}} F\left(u^{-}, v^{+}\right) \mathrm{d} x \\
= & I_{\infty}\left(u^{+}, v^{+}\right)+I_{\infty}\left(u^{-}, v^{-}\right) \geq m_{\infty}+m_{\infty}=2 m_{\infty},
\end{aligned}
$$

em que novamente utilizamos (2.54) e (2.56). Como $m_{\infty}$ é estritamente positivo pela Observação 2.2, acabamos de mostrar que não existe solução $(u, v)$ de $\left(\mathbb{S}_{\infty}\right)$ que muda de sinal em $\left[m_{\infty}, 2 m_{\infty}\right)$. Suponha $(u, v)$ solução de $\left(\mathbb{S}_{\infty}\right)$ tal que $I_{\infty}(u, v) \in\left(m_{\infty}, 2 m_{\infty}\right)$. Pela hipótese $(U)$ temos que é única ou qualquer solução positiva está no mesmo nível de energia do funcional associado a $\left(\mathbb{S}_{\infty}\right)$. Sem perda de generalidade, suponha $(u, v)$ solução positiva. Por [14], $(u, v)$ é radial e por $(U)$ segue que $(u, v)=\left(w_{1}, w_{2}\right)$ e $I_{\infty}(u, v)=$ $m_{\infty}$ ou $I_{\infty}(u, v)=m_{\infty}$ implicando em contradição com a hipótese de $I_{\infty}(u, v)>m_{\infty}$.

\subsection{Compacidade}

O próximo resultado que enunciaremos será fundamental para o estudo dos pontos críticos do funcional I. Ele descreve como uma sequência de Palais - Smale de $I$ se comporta assintoticamente.

Lema 2.10. (Lema de Splitting) Suponha que $\left\{\left(u_{n}, v_{n}\right)\right\}$ é uma sequência limitada em $H$ tal que

$$
I\left(u_{n}, v_{n}\right) \rightarrow d>0 \quad e \quad\left(\left.I\right|_{\mathcal{N}}\right)^{\prime}\left(u_{n}, v_{n}\right) \rightarrow 0 \quad \text { em } \quad H^{-1}
$$

Então, passando se necessário a uma subsequência, existem $\left(u_{0}, v_{0}\right)$ solução fraca de $(\mathbb{S})$, um número $k \in \mathbb{N} \cup\{0\}, k$ funções $\left(u^{1}, v^{1}\right), \ldots,\left(u^{k}, v^{k}\right) \in H$ soluções do problema limite $\left(\mathbb{S}_{\infty}\right)$ e $k$ sequências de pontos $\left\{y_{n}^{j}\right\}, 1 \leq j \leq k$ tais que

1. $\left(u_{n}, v_{n}\right) \rightarrow\left(u_{0}, v_{0}\right)$ em $H$ ou

2. $\left|y_{n}^{j}\right| \rightarrow+\infty$ e $\left|y_{n}^{j}-y_{n}^{i}\right| \rightarrow+\infty ; j \neq i$;

3. $\left(u_{n}, v_{n}\right)-\sum_{j=1}^{k}\left(u^{j}\left(\cdot-y_{n}^{j}\right), v^{j}\left(\cdot-y_{n}^{j}\right)\right) \rightarrow\left(u_{0}, v_{0}\right)$ em $H$;

4. $I\left(u_{n}, v_{n}\right) \rightarrow d=I\left(u_{0}, v_{0}\right)+\sum_{j=1}^{k} I_{\infty}\left(u^{j}, v^{j}\right)$. 
Demonstração: Inicialmente obtemos do Lema 2.7 que a sequência $\left\{\left(u_{n}, v_{n}\right)\right\}$ satisfaz $I^{\prime}\left(u_{n}, v_{n}\right) \rightarrow 0$. A prova segue como em [5] considerando que a não linearidade $F(u, v)$ é de classe $C^{2}(H)$ e seguindo os passos (2.11) e (2.46) a (2.55) da Proposição 2.31 em [20].

Como uma consequência dos Lemas 2.5 , 2.7 e 2.9 combinados com o Lema 2.10 temos o seguinte resultado

Lema 2.11. Suponha que o ínfimo $m$ definido em (2.5) não seja atingido. Então $m \geq m_{\infty} e$, ainda, o funcional I satisfaz a condição de Palais-Smale sobre $\mathcal{N}$ em qualquer nivel do intervalo $\left(m_{\infty}, 2 m_{\infty}\right)$.

Demonstração: Considere uma sequência $\left\{\left(u_{n}, v_{n}\right)\right\}$ tal que satisfaz $(P S)_{d}$ para o funcional $I$ restrito a $\mathcal{N}$. Então, a menos de subsequência, $\left\{\left(u_{n}, v_{n}\right)\right\}$ é limitada e é $(P S)_{d}$ para $I$ em todo espaco $H$ pelos Lemas 2.5 e 2.7 .

Suponha que o nível $m$ não seja atingido. Se $d=m$, então existe uma sequência $\left(u_{n}, v_{n}\right) \rightarrow\left(u_{0}, v_{0}\right)$, pela continuidade do funcional $I$, teríamos $I\left(u_{n}, v_{n}\right) \rightarrow I\left(u_{0}, v_{0}\right)=d=m$. Contradição com a hipótese neste caso. Logo, não existe tal sequência; o que implica que a possibilidade 1 no Lema de Splitting não ocorre. Decorre então que valem $(2),(3)$ e (4).

Em primeiro lugar, suponha que $\left(u_{0}, v_{0}\right)=(0,0)$, então por $(4)$,

$$
I\left(u_{n}, v_{n}\right) \rightarrow d=m=I\left(u_{0}, v_{0}\right)+\sum_{j=1}^{k} I_{\infty}\left(u^{j}, v^{j}\right) \geq 0+k I_{\infty}\left(w_{1}, w_{2}\right) \geq k m_{\infty} \geq m_{\infty}
$$

onde $\left(w_{1}, w_{2}\right)$ é a solução de energia mínima para $I_{\infty}$.

Em segundo lugar, assuma que $\left(u_{0}, v_{0}\right) \neq(0,0)$. Como $\left(u_{0}, v_{0}\right)$ pertence a $\mathcal{N}$, tem-se $I\left(u_{0}, v_{0}\right)>0$. Segue que $m>k m_{\infty} \geq m_{\infty}$. Logo, em ambos os casos, temos $m \geq m_{\infty}$ e assim a primeira parte do lema está verificada.

Por outro lado, seja $d \in\left(m_{\infty}, 2 m_{\infty}\right)$. Assim, para $n$ suficientemente grande,

$$
m_{\infty}<I\left(u_{n}, v_{n}\right)<2 m_{\infty}
$$

Se não existisse subsequência $\left\{\left(u_{n}, v_{n}\right)\right\}$ convergente, usando o Lema 2.10,

$$
I\left(u_{n}, v_{n}\right) \rightarrow d=I\left(u_{0}, v_{0}\right)+\sum_{j=1}^{k} I_{\infty}\left(u^{j}, v^{j}\right)
$$

Se $k \geq 2$, então

$$
\begin{aligned}
I\left(u_{n}, v_{n}\right) \rightarrow d & =I\left(u_{0}, v_{0}\right)+\sum_{j=1}^{k} I_{\infty}\left(u^{j}, v^{j}\right) \\
& \geq 0+k I_{\infty}\left(w_{1}, w_{2}\right) \geq k m_{\infty} \geq 2 m_{\infty}
\end{aligned}
$$

Isto configura um absurdo uma vez que $d<2 m_{\infty}$. Portanto, obrigatoriamente, tem-se $k=1$. Neste caso, se $\left(u_{0}, v_{0}\right)=(0,0)$, então

$$
2 m_{\infty}>I_{\infty}\left(u^{j}, v^{j}\right)=d>m_{\infty} .
$$


Isto é uma contradição com o Lema 2.9, pois não existe solução $\left(u^{j}, v^{j}\right)$ de $(\mathbb{S})_{\infty}$ no intervalo $\left(m_{\infty}, 2 m_{\infty}\right)$. Usando mais uma vez o Lema 2.10, como $\left(u_{0}, v_{0}\right)$ pertence a $\mathcal{N}$,

$$
\begin{aligned}
I\left(u_{n}, v_{n}\right) \rightarrow d & =I\left(u_{0}, v_{0}\right)+1 . I_{\infty}\left(u^{j}, v^{j}\right) \\
& \geq m+I_{\infty}\left(w_{1}, w_{2}\right)=m+m_{\infty} \geq m_{\infty}+m_{\infty}=2 m_{\infty} .
\end{aligned}
$$

Isto também configura uma contradição, visto que $d<2 m_{\infty}$. Neste caso, concluimos que $k$ não pode ser maior ou igual a 1 e devemos ter $k=0$ e, portanto, por (3) existe uma subsequência de $\left\{\left(u_{n}, v_{n}\right)\right\}$ convergente e o lema está provado.

\subsection{Estimativas assintóticas}

Visto que problema limite $\left(\mathbb{S}_{\infty}\right)$ tem uma solução $\left(w_{1}, w_{2}\right)$ positiva de energia mínima, trabalharemos com esta solução transladada, isto é,

$$
w_{i}^{R y_{0}}:=w_{i}\left(x-R y_{0}\right) \quad \text { e } \quad w_{i}^{R y}:=w_{i}(x-R y)
$$

para $i=1,2$. Seja $\beta \in[0,1], R>0, y_{0} \in \mathbb{R}^{N}$ fixado, $y \in \partial B_{2}\left(y_{0}\right)$ e definimos a combinação linear

$$
Z_{\beta, y}^{R}:=\beta\left(w_{1}^{R y_{0}}, w_{2}^{R y_{0}}\right)+(1-\beta)\left(w_{1}^{R y}, w_{2}^{R y}\right)=\left(\beta w_{1}^{R y_{0}}+(1-\beta) w_{1}^{R y}, \beta w_{2}^{R y_{0}}+(1-\beta) w_{2}^{R y}\right) .
$$

Novamente iremos precisar do resultado técnico em [1]. Por comodidade, vamos enunciá-lo novamente.

Lema 2.12. Assuma que $\mu_{2}>\mu_{1} \geq 0$. Então, existe um número $C>0$ tal que, para todo $x_{1}, x_{2} \in \mathbb{R}^{N}$,

$$
\int_{\mathbb{R}^{N}} e^{-\mu_{1}\left|x-x_{1}\right|} e^{-\mu_{2}\left|x-x_{2}\right|} \mathrm{d} x \leq C e^{-\mu_{1}\left|x_{1}-x_{2}\right|} .
$$

Se $\mu_{2} \geq \mu_{1}>0$ e $\mu_{3}>\mu_{1} \geq 0$. Então, existe um número $C>0$ tal que, para quaisquer $x_{1}, x_{2}, x_{3} \in$ $\mathbb{R}^{N}$,

$$
\int_{\mathbb{R}^{N}} e^{-\mu_{1}\left|x-x_{1}\right|} e^{-\mu_{2}\left|x-x_{2}\right|} e^{-\mu_{3}\left|x-x_{3}\right|} \mathrm{d} x \leq C e^{-\frac{\mu_{1}}{2}\left(\left|x_{1}-x_{2}\right|+\left|x_{1}-x_{3}\right|+\left|x_{2}-x_{3}\right|\right)} .
$$

O próximo resultado garante que a variedade $\mathcal{N}$ é não vazia.

Lema 2.13. Existem números $R_{0}>0, T_{0}>2$ e para cada $R \geq R_{0}, y \in \partial B_{2}\left(y_{0}\right)$ e $0 \leq \beta \leq 1$, um único $T_{\beta, y}^{R}>0$ satisfazendo

$$
T_{\beta, y}^{R} Z_{\beta, y}^{R} \in \mathcal{N}
$$

Ademais, $T_{\beta, y}^{R} \in\left[0, T_{0}\right)$ e $T_{\beta, y}^{R}$ é uma função contínua nas variáveis $\beta, y, R$. 
Demonstração: Sejam $r, u_{1}, u_{2}, v_{1}, v_{2}>0$. Escrevendo a combinação $\left(r\left(u_{1}+u_{2}\right), r\left(v_{1}+v_{2}\right)\right)$ e aplicando o funcional $J_{\infty}$ nesta combinação temos

$$
\begin{aligned}
J_{\infty}\left(r\left(u_{1}+u_{2}\right), r\left(v_{1}+v_{2}\right)\right)= & \left\|\left(r u_{1}+r u_{2}, r v_{1}+r v_{2}\right)\right\|^{2}-2 \lambda \int_{\mathbb{R}^{N}} r^{2}\left(u_{1}+u_{2}\right)\left(v_{1}+v_{2}\right) \mathrm{d} x \\
& -\int_{\mathbb{R}^{N}} \nabla F\left(r u_{1}+r u_{2}, r v_{1}+r v_{2}\right)\left(r u_{1}+r u_{2}, r v_{1}+r v_{2}\right) \mathrm{d} x \\
= & r^{2}\left\|u_{1}\right\|^{2}+r^{2}\left\|u_{2}\right\|^{2}+r^{2}\left\|v_{1}\right\|^{2}+r^{2}\left\|v_{2}\right\|^{2}+2 r^{2}\left\langle u_{1}, u_{2}\right\rangle+2 r^{2}\left\langle v_{1}, v_{2}\right\rangle \\
& -2 \lambda \int_{\mathbb{R}^{N}} r^{2}\left(u_{1} v_{1}+u_{1} v_{2}+u_{2} v_{1}+u_{2} v_{2}\right) \mathrm{d} x \\
& -r^{2} \int_{\mathbb{R}^{N}} \frac{\nabla F\left(r u_{1}+r u_{2}, r v_{1}+r v_{2}\right)}{r}\left(u_{1}+u_{2}, v_{1}+v_{2}\right) \mathrm{d} x \\
= & r^{2}\left\|u_{1}\right\|^{2}+r^{2}\left\|u_{2}\right\|^{2}+r^{2}\left\|v_{1}\right\|^{2}+r^{2}\left\|v_{2}\right\|^{2}+2 r^{2}\left\langle u_{1}, u_{2}\right\rangle+2 r^{2}\left\langle v_{1}, v_{2}\right\rangle \\
& -2 \lambda r^{2} \int_{\mathbb{R}^{N}}\left(u_{1} v_{1}+u_{1} v_{2}+u_{2} v_{1}+u_{2} v_{2}\right) \mathrm{d} x \\
& -r^{2} \int_{\mathbb{R}^{N}} \frac{\nabla F\left(r u_{1}+r u_{2}, r v_{1}+r v_{2}\right)}{r}\left(u_{1}, v_{1}\right) \mathrm{d} x \\
& -r^{2} \int_{\mathbb{R}^{N}} \frac{\nabla F\left(r u_{1}+r u_{2}, r v_{1}+r v_{2}\right)}{r}\left(u_{2}, v_{2}\right) \mathrm{d} x .
\end{aligned}
$$

Por $\left(F_{1}\right)$ segue que

$$
\begin{aligned}
\frac{J_{\infty}\left(r\left(u_{1}+u_{2}\right), r\left(v_{1}+v_{2}\right)\right)}{r^{2} \leq} & \left\|u_{1}\right\|^{2}+\left\|u_{2}\right\|^{2}+\left\|v_{1}\right\|^{2}+\left\|v_{2}\right\|^{2}+2\left\langle u_{1}, u_{2}\right\rangle+2\left\langle v_{1}, v_{2}\right\rangle \\
& -2 \lambda \int_{\mathbb{R}^{N}}\left(u_{1} v_{1}+u_{2} v_{2}\right) \mathrm{d} x-\int_{\mathbb{R}^{N}} \frac{\nabla F\left(r u_{1}, r v_{1}\right)}{r}\left(u_{1}, v_{1}\right) \mathrm{d} x \\
& -\int_{\mathbb{R}^{N}} \frac{\nabla F\left(r u_{2}, r v_{2}\right)}{r}\left(u_{2}, v_{2}\right) \mathrm{d} x .
\end{aligned}
$$

Tomando $u_{1}=\beta w_{1}^{R y_{0}}, u_{2}=(1-\beta) w_{1}^{R y}, v_{1}=\beta w_{2}^{R y_{0}}, v_{2}=(1-\beta) w_{2}^{R y}$ em $(2.58)$,

$$
\begin{aligned}
& \frac{J_{\infty}\left(r \beta w_{1}^{R y_{0}}+r(1-\beta) w_{1}^{R y}, r \beta w_{2}^{R y_{0}}+r(1-\beta) w_{2}^{R y}\right)}{r^{2}} \\
& \leq\left\|\beta w_{1}^{R y_{0}}\right\|^{2}+\left\|(1-\beta) w_{1}^{R y}\right\|^{2}+\left\|\beta w_{2}^{R y_{0}}\right\|^{2}+\left\|(1-\beta) w_{2}^{R y}\right\|^{2}+2\left\langle\beta w_{1}^{R y_{0}},(1-\beta) w_{1}^{R y}\right\rangle \\
& \quad+2\left\langle\beta w_{2}^{R y_{0}},(1-\beta) w_{2}^{R y}\right\rangle-2 \lambda \int_{\mathbb{R}^{N}}\left(\beta w_{1}^{R y_{0}} \beta w_{2}^{R y_{0}}+(1-\beta) w_{1}^{R y}(1-\beta) w_{2}^{R y}\right) \mathrm{d} x \\
& \quad-\int_{\mathbb{R}^{N}} \frac{\nabla F\left(r \beta w_{1}^{R y_{0}}, r \beta w_{2}^{R y_{0}}\right)}{r}\left(\beta w_{1}^{R y_{0}}, \beta w_{2}^{R y_{0}}\right) \mathrm{d} x \\
& \quad-\int_{\mathbb{R}^{N}} \frac{\nabla F\left(r(1-\beta) w_{1}^{R y}, r(1-\beta) w_{2}^{R y}\right)}{r}\left((1-\beta) w_{1}^{R y},(1-\beta) w_{2}^{R y}\right) \mathrm{d} x
\end{aligned}
$$




$$
\begin{aligned}
= & \beta^{2}\left\{\left\|w_{1}^{R y_{0}}\right\|^{2}+\left\|w_{2}^{R y_{0}}\right\|^{2}-2 \lambda \int_{\mathbb{R}^{N}}\left(w_{1}^{R y_{0}} w_{2}^{R y_{0}}\right) \mathrm{d} x\right\}-\beta \int_{\mathbb{R}^{N}} \frac{\nabla F\left(r \beta w_{1}^{R y_{0}}, r \beta w_{2}^{R y_{0}}\right)}{r}\left(w_{1}^{R y_{0}}, w_{2}^{R y_{0}}\right) \mathrm{d} x \\
& +(1-\beta)^{2}\left\{\left\|w_{1}^{R y}\right\|^{2}+\left\|w_{2}^{R y}\right\|^{2}-2 \lambda \int_{\mathbb{R}^{N}}\left(w_{1}^{R y} w_{2}^{R y}\right) \mathrm{d} x\right\} \\
& -(1-\beta) \int_{\mathbb{R}^{N}} \frac{\nabla F\left(r(1-\beta) w_{1}^{R y}, r(1-\beta) w_{2}^{R y}\right)}{r}\left(w_{1}^{R y}, w_{2}^{R y}\right) \mathrm{d} x \\
& +2\left\langle\beta w_{1}^{R y_{0}},(1-\beta) w_{1}^{R y}\right\rangle+2\left\langle\beta w_{2}^{R y_{0}},(1-\beta) w_{2}^{R y}\right\rangle \\
= & \beta^{2} \int_{\mathbb{R}^{N}} \nabla F\left(w_{1}^{R y_{0}}, w_{2}^{R y_{0}}\right)\left(w_{1}^{R y_{0}}, w_{2}^{R y_{0}}\right) \mathrm{d} x-\beta \int_{\mathbb{R}^{N}} \frac{\nabla F\left(r \beta w_{1}^{R y_{0}}, r \beta w_{2}^{R y_{0}}\right)}{r}\left(\beta w_{1}^{R y_{0}}, \beta w_{2}^{R y_{0}}\right) \mathrm{d} x \\
& +(1-\beta)^{2} \int_{\mathbb{R}^{N}} \nabla F\left(w_{1}^{R y}, w_{2}^{R y}\right)\left(w_{1}^{R y}, w_{2}^{R y}\right) \mathrm{d} x \\
& -(1-\beta) \int_{\mathbb{R}^{N}} \frac{\nabla F\left(r(1-\beta) w_{1}^{R y}, r(1-\beta) w_{2}^{R y}\right)}{r}\left(w_{1}^{R y}, w_{2}^{R y}\right) \mathrm{d} x \\
& +2\left\langle\beta w_{1}^{R y_{0}},(1-\beta) w_{1}^{R y}\right\rangle+2\left\langle\beta w_{2}^{R y_{0}},(1-\beta) w_{2}^{R y}\right\rangle \\
= & \beta^{2} \int_{\mathbb{R}^{N}} \nabla F\left(w_{1}^{R y_{0}}, w_{2}^{R y_{0}}\right)\left(w_{1}^{R y_{0}}, w_{2}^{R y_{0}}\right) \mathrm{d} x-\beta^{2} \int_{\mathbb{R}^{N}} \frac{\nabla F\left(r \beta w_{1}^{R y_{0}}, r \beta w_{2}^{R y_{0}}\right)}{r \beta}\left(w_{1}^{R y_{0}}, w_{2}^{R y_{0}}\right) \mathrm{d} x \\
& +(1-\beta)^{2} \int_{\mathbb{R}^{N}} \nabla F\left(w_{1}^{R y}, w_{2}^{R y}\right)\left(w_{1}^{R y}, w_{2}^{R y}\right) \mathrm{d} x \\
& -(1-\beta)^{2} \int_{\mathbb{R}^{N}} \frac{\nabla F\left(r(1-\beta) w_{1}^{R y}, r(1-\beta) w_{2}^{R y}\right)}{r(1-\beta)}\left(w_{1}^{R y}, w_{2}^{R y}\right) \mathrm{d} x \\
& +2\left\langle\beta w_{1}^{R y_{0}},(1-\beta) w_{1}^{R y}\right\rangle+2\left\langle\beta w_{2}^{R y_{0}},(1-\beta) w_{2}^{R y}\right\rangle .
\end{aligned}
$$

Considere a aplicação

$$
\Gamma(\beta, r)=\beta^{2}\left\{\int_{\mathbb{R}^{N}} \nabla F(u, v)(u, v) \mathrm{d} x-\int_{\mathbb{R}^{N}} \frac{\nabla F(r \beta u, r \beta v)}{r \beta}(u, v) \mathrm{d} x\right\} .
$$

Por $\left(F_{1}\right), \Gamma(\beta, r)$ é decrescente em relação a $\beta$ e $r$. Argumentando como no caso escalar, confira Proposição 1.1, existirá um $T_{0}>2$ e $S_{0}<0$ tais que

$$
\Gamma(\beta, r)+\Gamma(1-\beta, r)<S_{0}<0, \forall r \geq T_{0}, \forall \beta \in[0,1] .
$$

Além disso, $T_{0}$ e $S_{0}$ independem de $R$ pela invariância por translações das integrais em $\mathbb{R}^{N}$.

Usando (2.60) em (2.59) segue que

$$
\begin{aligned}
& \frac{J_{\infty}\left(r \beta w_{1}^{R y_{0}}+r(1-\beta) w_{1}^{R y}, r \beta w_{2}^{R y_{0}}+r(1-\beta) w_{2}^{R y}\right)}{r^{2}} \\
& \leq \Gamma(\beta, r)+\Gamma(1-\beta, r)+2\left\langle w_{1}^{R y_{0}}, w_{1}^{R y}\right\rangle+2\left\langle w_{2}^{R y_{0}}, w_{2}^{R y}\right\rangle \\
& \leq \Gamma(\beta, r)+\Gamma(1-\beta, r)+o_{R}(1)<S_{0}+o_{R}(1) .
\end{aligned}
$$


Desde que podemos escrever para $(u, v)$ em $H$

$$
J(u, v)=J_{\infty}(u, v)-\int_{\mathbb{R}^{N}} a(x) \nabla F(u, v)(u, v) \mathrm{d} x
$$

segue que

$$
\begin{gathered}
\frac{J\left(r \beta w_{1}^{R y_{0}}+r(1-\beta) w_{1}^{R y}, r \beta w_{2}^{R y_{0}}+r(1-\beta) w_{2}^{R y}\right)}{r^{2}} \\
=\frac{J_{\infty}\left(r \beta w_{1}^{R y_{0}}+r(1-\beta) w_{1}^{R y}, r \beta w_{2}^{R y_{0}}+r(1-\beta) w_{2}^{R y}\right)}{r^{2}} \\
\quad-\frac{1}{r^{2}} \int_{\mathbb{R}^{N}}\left[a(x) \nabla F\left(r \beta w_{1}^{R y_{0}}+r(1-\beta) w_{1}^{R y}, r \beta w_{2}^{R y_{0}}+r(1-\beta) w_{2}^{R y}\right)\right. \\
\left.\quad\left(r \beta w_{1}^{R y_{0}}+r(1-\beta) w_{1}^{R y}, r \beta w_{2}^{R y_{0}}+r(1-\beta) w_{2}^{R y}\right)\right] \mathrm{d} x .
\end{gathered}
$$

Como

$$
\nabla F(u, v)(u, v)=\frac{\left(u^{2}+v^{2}\right)^{2}}{1+s\left(u^{2}+v^{2}\right)} \leq C\left(u^{4}+v^{4}\right)
$$

e ainda, pelo Lema 2.12 e $\left(a_{2}\right)$,

$$
\int_{\mathbb{R}^{N}}|a(x)|\left(w_{i}^{R y_{0}}\right)^{4} \mathrm{~d} x \leq C \int_{\mathbb{R}^{N}} e^{-k|x|} e^{-4 \sqrt{1-\lambda}\left|x-R y_{0}\right|} \mathrm{d} x \leq C e^{-k\left|R y_{0}\right|}=o_{R}(1)
$$

e

$$
\int_{\mathbb{R}^{N}}|a(x)|\left(w_{i}^{R y}\right)^{4} \mathrm{~d} x \leq C \int_{\mathbb{R}^{N}} e^{-k|x|} e^{-4 \sqrt{1-\lambda}|x-R y|} \mathrm{d} x \leq C e^{-k|R y|}=o_{R}(1),
$$

substituindo em (2.62) resulta que

$$
\begin{aligned}
& \frac{J\left(r\left(\beta w_{1}^{R y_{0}}+(1-\beta) w_{1}^{R y}\right), r\left(\beta w_{2}^{R y_{0}}+(1-\beta) w_{2}^{R y}\right)\right)}{r^{2}} \\
& \leq \frac{J_{\infty}\left(r \beta w_{1}^{R y_{0}}+r(1-\beta) w_{1}^{R y}, r \beta w_{2}^{R y_{0}}+r(1-\beta) w_{2}^{R y}\right)}{r^{2}}+o_{R}(1) \\
& <S_{0}+o_{R}(1)<0,
\end{aligned}
$$

para $R$ suficientemente grande e $r \geqslant T_{0}, 0 \leq \beta \leq 1$ e $y \in \partial B_{2}\left(y_{0}\right)$. Para concluirmos nosso resultado, basta combinarmos (2.63) com (2.3) para garantir que existe um único $r=T_{\beta, y}^{R}>0$ tal que $T_{\beta, y}^{R} Z_{\beta, y}^{R} \in \mathcal{N}$. Consequentemente, $\mathcal{N}$ é não vazia. Argumentando como no Lema 1.10, demonstra-se a continuidade de $T_{\lambda, y}^{R}$ nas variáveis $\lambda, y, R$ e o lema está provado.

Definimos a quantidade

$$
\varepsilon_{R}:=\int_{\mathbb{R}^{N}} \nabla F\left(w_{1}\left(x-R y_{0}\right), w_{2}\left(x-R y_{0}\right)\right)\left(w_{1}(x-R y), w_{2}(x-R y)\right) \mathrm{d} x,
$$

onde $\left(w_{1}, w_{2}\right)$ é uma solução positiva radial de $\left(\mathbb{S}_{\infty}\right)$.

O próximo resultado é essencial para provarmos o comportamento assintótico da quantidade $\varepsilon_{R}$. O Lema já foi utilizado no caso escalar e vamos enunciá-lo novamente por comodidade. 
Lema 2.14. Sejam $\varphi \in C\left(\mathbb{R}^{N}\right) \cap L^{\infty}\left(\mathbb{R}^{N}\right), \psi \in C\left(\mathbb{R}^{N}\right)$ satisfazendo para constantes a, $b \geq 0$ e $c \in \mathbb{R}$,

$$
\varphi(x) e^{a|x|}|x|^{b} \rightarrow c ; \quad \text { se }|x| \rightarrow+\infty
$$

$e$

$$
\int_{\mathbb{R}^{N}}|\psi(x)| e^{a|x|}\left(1+|x|^{b}\right) \mathrm{d} x<+\infty
$$

Então

$$
\lim _{|\bar{y}| \rightarrow+\infty}\left[\left(\int_{\mathbb{R}^{N}} \varphi(x+\bar{y}) \psi(x) \mathrm{d} x\right) e^{a|\bar{y}|}|\bar{y}|^{b}-c \int_{\mathbb{R}^{N}} e^{-\frac{a\langle x, \bar{y}\rangle}{|\bar{y}|}} \psi(x) \mathrm{d} x\right]=0 .
$$

Demonstração: Segue de [8] e está demonstrado no Capítulo 1, Lema 1.11.

Nos dois lemas a seguir, iremos estimar a quantidade $\varepsilon_{R}$, isto é, mostrar decaimento exato desta quantidade $\varepsilon_{R}$.

Lema 2.15. Considere $y \in \partial B_{2}\left(y_{0}\right)$ com $y_{0} \in \mathbb{R}^{N}$ fixado tal que $\left\|y_{0}\right\|=1$. Então, existe uma constante $C_{0}>0$ tal que

$$
\lim _{R \rightarrow+\infty} \varepsilon_{R}(2 R)^{\frac{N-1}{2}} e^{2 \sqrt{1-\lambda} R}=C_{0}
$$

Demonstração: Pela definição de $\varepsilon_{R}$ em (2.64) tem-se

$$
\begin{aligned}
\varepsilon_{R} & =\int_{\mathbb{R}^{N}} \nabla F\left(w_{1}\left(x-R y_{0}\right), w_{2}\left(x-R y_{0}\right)\right)\left(w_{1}(x-R y), w_{2}(x-R y)\right) \mathrm{d} x \\
& =\int_{\mathbb{R}^{N}} \frac{\left(w_{1}^{R y_{0}}\right)^{2}+\left(w_{2}^{R y_{0}}\right)^{2}}{1+s\left(\left(w_{1}^{R y_{0}}\right)^{2}+\left(w_{2}^{R y_{0}}\right)^{2}\right)}\left(w_{1}^{R y_{0}} w_{1}^{R y}+w_{2}^{R y_{0}} w_{2}^{R y}\right) \mathrm{d} x \\
& =\int_{\mathbb{R}^{N}} \frac{w_{1}^{2}+w_{2}^{2}}{1+s\left(w_{1}^{2}+w_{2}^{2}\right)} w_{1} w_{1}^{R\left(y-y_{0}\right)} \mathrm{d} x+\int_{\mathbb{R}^{N}} \frac{w_{1}^{2}+w_{2}^{2}}{1+s\left(w_{1}^{2}+w_{2}^{2}\right)} w_{2} w_{2}^{R\left(y-y_{0}\right)} \mathrm{d} x .
\end{aligned}
$$

Para estimar cada integral em (2.69), iremos utilizar o Lema 2.14 com

$$
\psi=\frac{w_{1}^{2}+w_{2}^{2}}{1+s\left(w_{1}^{2}+w_{2}^{2}\right)} w_{1}, \varphi=w_{1}^{R\left(y-y_{0}\right)}, \bar{y}=-R\left(y-y_{0}\right), a=\sqrt{1-\lambda} \text { e } b=\frac{N-1}{2} .
$$

Como $\left(w_{1}, w_{2}\right)$ é solução do problema $\left(\mathbb{S}_{\infty}\right)$, pelo Teorema 2.1 existe um $R_{0}>0$ tal que para todo $|x|>R_{0}$ obtemos (2.65) para alguma constante positiva $c$. Agora vamos verificar (2.66) e, para isso note que para todo $|x|>R_{1} \geq R_{0}$, tem-se 


$$
\begin{aligned}
\int_{\mathbb{R}^{N^{N}}}|\psi(x)| e^{\alpha|x|}\left(1+|x|^{\beta}\right) \mathrm{d} x= & \int_{\mathbb{R}^{N}} \frac{w_{1}^{2}+w_{2}^{2}}{1+s\left(w_{1}^{2}+w_{2}^{2}\right)} w_{1} e^{\sqrt{1-\lambda}|x|}\left(1+|x|^{\frac{N-1}{2}}\right) \mathrm{d} x \\
= & \int_{B_{R_{1}}(0)} \frac{w_{1}^{2}+w_{2}^{2}}{1+s\left(w_{1}^{2}+w_{2}^{2}\right)} w_{1} e^{\sqrt{1-\lambda}|x|}\left(1+|x|^{\frac{N-1}{2}}\right) \mathrm{d} x \\
& +\int_{\mathbb{R}^{N} \backslash B_{R_{1}}(0)} \frac{w_{1}^{2}+w_{2}^{2}}{1+s\left(w_{1}^{2}+w_{2}^{2}\right)} w_{1} e^{\sqrt{1-\lambda}|x|}\left(1+|x|^{\frac{N-1}{2}}\right) \mathrm{d} x \\
\leq & C \mu\left(B_{R_{1}}(0)\right)+C \int_{\mathbb{R}^{N} \backslash B_{R_{1}}(0)}|x|^{-3 \frac{N-1}{2}} e^{-3 \sqrt{1-\lambda}|x|} e^{\sqrt{1-\lambda}|x|}\left(1+|x|^{\frac{N-1}{2}}\right) \mathrm{d} x \\
\leq & C \mu\left(B_{R_{1}}(0)\right)+C \int_{\mathbb{R}^{N} \backslash B_{R_{1}}(0)}|x|^{-3 \frac{N-1}{2}} e^{-2 \sqrt{1-\lambda}|x|}\left(1+|x|^{\frac{N-1}{2}}\right) \mathrm{d} x<\infty,
\end{aligned}
$$

onde $\mu\left(B_{R_{1}}(0)\right)$ é a medida de Lebesgue da bola de raio $R_{1}$ centrada na origem. Assim, por (2.70), está verificado (2.66). Podemos então utilizar o Lema 2.14 e o limite (2.67), para provar que, para todo $|x|>R_{2} \operatorname{com} R_{2}>\max \left\{R_{0}, R_{1}\right\}$,

$$
\lim _{R \rightarrow+\infty}\left[\left(\int_{\mathbb{R}^{N}} \frac{w_{1}^{2}+w_{2}^{2}}{1+s\left(w_{1}^{2}+w_{2}^{2}\right)} w_{1} w_{1}^{R\left(y-y_{0}\right)} \mathrm{d} x\right) e^{\sqrt{1-\lambda}\left|R\left(y-y_{0}\right)\right|}\left|R\left(y-y_{0}\right)\right|^{\frac{N-1}{2}}\right]=C>0 .
$$

De forma inteiramente análoga, obtemos

$$
\lim _{R \rightarrow+\infty}\left[\left(\int_{\mathbb{R}^{N}} \frac{w_{1}^{2}+w_{2}^{2}}{1+s\left(w_{1}^{2}+w_{2}^{2}\right)} w_{2} w_{2}^{R\left(y-y_{0}\right)} \mathrm{d} x\right) e^{\sqrt{1-\lambda}\left|R\left(y-y_{0}\right)\right|}\left|R\left(y-y_{0}\right)\right|^{\frac{N-1}{2}}\right]=C>0 .
$$

De (2.71), (2.72) e (2.69) segue (2.68), e o lema está provado .

Apresentaremos uma estimativa inferior para $\varepsilon_{R}$.

Lema 2.16. Existe uma constante $\bar{C}_{0}>0$ tal que para todo $t, s \geqslant \frac{1}{2}, y \in \partial B_{2}\left(y_{0}\right)$ com $y_{0} \in \mathbb{R}^{N}$ fixado e $R$ suficientemente grande,

$$
\int_{\mathbb{R}^{N}} \nabla F\left(t w_{1}\left(x-R y_{0}\right), t w_{2}\left(x-R y_{0}\right)\right)\left(s w_{1}(x-R y), s w_{2}(x-R y)\right) \mathrm{d} x \geq \bar{C}_{0}(R)^{-\frac{N-1}{2}} e^{-2 \sqrt{1-\lambda} R} .
$$

Demonstração: Segue do Teorema 2.1 que existem constantes $C_{1}>0$ e $C_{2}>0$ tais que

$$
C_{1}(1+|x|)^{-\frac{N-1}{2}} e^{-\sqrt{1-\lambda}|x|} \leq w_{i}(x) \leq C_{2}(1+|x|)^{-\frac{N-1}{2}} e^{-\sqrt{1-\lambda}|x|},
$$

para $i=1,2$ e para $x \in \mathbb{R}^{N}$. Para $|x|<1$ e para todo $y$ tal que $\left|y-y_{0}\right|=2$ e considerando $R>1$, note que

$$
1+\left|x-R\left(y-y_{0}\right)\right| \leq 1+|x|+R\left|y-y_{0}\right| \leq R+R+2 R=4 R .
$$

Segue por (2.73) que existe uma constante $C>0$ tal que

$$
\begin{aligned}
w\left(x-R\left(y-y_{0}\right)\right) & \geq C_{1}\left(1+\left|x-R\left(y-y_{0}\right)\right|\right)^{-\frac{N-1}{2}} e^{-\sqrt{1-\lambda}\left|x-R\left(y-y_{0}\right)\right|} \\
& \geq C_{1}(4 R)^{-\frac{N-1}{2}} e^{-\sqrt{1-\lambda}|x|} e^{-2 \sqrt{1-\lambda} R} \\
& \geq C(2 R)^{-\frac{N-1}{2}} e^{-\sqrt{1-\lambda}} e^{-2 \sqrt{1-\lambda} R} \\
& =C(2 R)^{-\frac{N-1}{2}} e^{-2 \sqrt{1-\lambda} R} .
\end{aligned}
$$


Usando (2.74) existe uma constante $C>0$ tal que

$$
\begin{aligned}
& \int_{\mathbb{R}^{N}} \nabla F\left(t w_{1}\left(x-R y_{0}\right), t w_{2}\left(x-R y_{0}\right)\right)\left(s w_{1}(x-R y), s w_{2}(x-R y)\right) \mathrm{d} x \\
= & s \int_{\mathbb{R}^{N}} \nabla F\left(t w_{1}\left(x-R y_{0}\right), t w_{2}\left(x-R y_{0}\right)\right)\left(w_{1}(x-R y), w_{2}(x-R y)\right) \mathrm{d} x \\
= & s \int_{\mathbb{R}^{N}} \frac{\left(t w_{1}^{R y_{0}}\right)^{2}+\left(t w_{2}^{R y_{0}}\right)^{2}}{1+s\left(\left(t w_{1}^{R y_{0}}\right)^{2}+\left(t w_{2}^{R y_{0}}\right)^{2}\right)}\left(t w_{1}^{R y_{0}} w_{1}^{R y}+t w_{2}^{R y_{0}} w_{2}^{R y}\right) \mathrm{d} x \\
\geq & \frac{1}{4} \int_{\mathbb{R}^{N}} \frac{\left(t w_{1}^{R y_{0}}\right)^{2}+\left(t w_{2}^{R y_{0}}\right)^{2}}{1+s\left(\left(t w_{1}^{R y_{0}}\right)^{2}+\left(t w_{2}^{R y_{0}}\right)^{2}\right)}\left(w_{1}^{R y_{0}} w_{1}^{R y}+w_{2}^{R y_{0}} w_{2}^{R y}\right) \mathrm{d} x \\
\geq & \frac{1}{4} \int_{B_{1}\left(R y_{0}\right)} \frac{\left(t w_{1}^{R y_{0}}\right)^{2}+\left(t w_{2}^{R y_{0}}\right)^{2}}{1+s\left(\left(t w_{1}^{R y_{0}}\right)^{2}+\left(t w_{2}^{R y_{0}}\right)^{2}\right.}\left(w_{1}^{R y_{0}} w_{1}^{R y}+w_{2}^{R y_{0}} w_{2}^{R y}\right) \mathrm{d} x \\
\geq & \frac{1}{4} \min _{B_{1}(0)}\left\{\frac{\left(t w_{1}(x)\right)^{2}+\left(t w_{2}(x)\right)^{2}}{1+s\left(\left(t w_{1}(x)\right)^{2}+\left(t w_{2}(x)\right)^{2}\right)}\right\} \int_{B_{1}(0)}\left(w_{1}(x) w_{1}^{R\left(y-y_{0}\right)}+w_{2}(x) w_{2}^{R\left(y-y_{0}\right)}\right) \mathrm{d} x \\
\geq & \bar{C}_{0}(2 R)^{-\frac{N-1}{2}} e^{-2 \sqrt{1-\lambda} R} \cdot
\end{aligned}
$$

Para nossas finas estimativas de energia precisaremos utilizar os dois próximos resultados técnicos.

Corolário 2.17. Existe uma constante positiva $C$ tal que

$$
\begin{aligned}
& \left|\int_{\mathbb{R}^{N}}\left(t f\left(w_{1}^{R y_{0}}, w_{2}^{R y_{0}}\right)-f\left(t w_{1}^{R y_{0}}, t w_{2}^{R y_{0}}\right)\right) w_{1}^{R y} \mathrm{~d} x\right| \\
& +\left|\int_{\mathbb{R}^{N}}\left(t g\left(w_{1}^{R y_{0}}, w_{2}^{R y_{0}}\right)-g\left(t w_{1}^{R y_{0}}, t w_{2}^{R y_{0}}\right)\right) w_{2}^{R y} \mathrm{~d} x\right| \leq C|t-1| O\left(\varepsilon_{R}\right),
\end{aligned}
$$

uniformemente em $y \in \partial B_{2}\left(y_{0}\right), t \in[0, a] ; \quad a>1$ e $R$ suficientemente grande.

Demonstração: Defina as funções reais $h_{1}(t):=t f(u, v)-f(t u, t v)$ e $h_{2}(t):=t g(u, v)-g(t u, t v)$. Observe que $h_{1}(1)=f(u, v)-f(u, v)=0$ e $h_{2}(1)=g(u, v)-g(u, v)=0$, para todo $u$ e $v$. Além, do fato que $f(t u, t v)=\frac{t^{3}\left(u^{2}+v^{2}\right) u}{1+s t^{2}\left(u^{2}+v^{2}\right)}$, fixados $u$ e $v$ e derivando $f(t u, t v)$ em relação a $t$ obtemos

$$
\begin{aligned}
\frac{d}{d t} f(t u, t v) & =\frac{d}{d t}\left(\frac{t^{3}\left(u^{2}+v^{2}\right) u}{1+s t^{2}\left(u^{2}+v^{2}\right)}\right) \\
& =\frac{3 t^{2}\left(u^{2}+v^{2}\right) u\left(1+s t^{2}\left(u^{2}+v^{2}\right)\right)-t^{3}\left(u^{2}+v^{2}\right)(u 2 s t)\left(u^{2}+v^{2}\right)}{\left(1+s t^{2}\left(u^{2}+v^{2}\right)\right)^{2}} \\
& =\frac{3 t^{2}\left(u^{2}+v^{2}\right) u+3 s t^{4}\left(u^{2}+v^{2}\right)^{2} u-2 s t^{4}\left(u^{2}+v^{2}\right)^{2} u}{\left(1+s t^{2}\left(u^{2}+v^{2}\right)\right)^{2}} \\
& =\frac{3 t^{2}\left(u^{2}+v^{2}\right) u+s t^{4}\left(u^{2}+v^{2}\right)^{2} u}{\left(1+s t^{2}\left(u^{2}+v^{2}\right)\right)^{2}} .
\end{aligned}
$$


Usando (2.75) obtemos

$$
\begin{aligned}
\left|h_{1}^{\prime}(t)\right| & \leq|f(u, v)|+\left|\frac{d}{d t} f(t u, t v)\right| \\
& \leq|f(u, v)|+3 t^{2}\left(u^{2}+v^{2}\right)|u|+s t^{4}\left(u^{2}+v^{2}\right)^{2}|u| \\
& \leq|f(u, v)|+\max \left\{3 t^{2}, s t^{4}\right\}\left\{\left(u^{2}+v^{2}\right)|u|+\left(u^{2}+v^{2}\right)^{2}|u|\right\} .
\end{aligned}
$$

Suponha, sem perda de generalidade, $t>1$ e utilizando o Teorema do Valor Médio para $h_{1}$, existe um $\tau$ entre $t$ e 1 tal que

$$
\left|h_{1}(t)-h_{1}(1)\right| \leq\left|h_{1}^{\prime}(\tau)\right||t-1| \leq|t-1|\left\{|f(u, v)|+C\left(\left(u^{2}+v^{2}\right) u+\left(u^{2}+v^{2}\right)^{2} u\right)\right\} .
$$

Similarmente obtemos

$$
\frac{d}{d t} g(t u, t v)=\frac{3 t^{2}\left(u^{2}+v^{2}\right) v+s t^{4}\left(u^{2}+v^{2}\right)^{2} v}{\left(1+s t^{2}\left(u^{2}+v^{2}\right)\right)^{2}}
$$

e

$$
\left|h_{2}(t)-h_{2}(1)\right| \leq|t-1|\left\{|g(u, v)|+C\left(\left(u^{2}+v^{2}\right)|v|+\left(u^{2}+v^{2}\right)^{2}|v|\right)\right\}
$$

Tomando $u=w_{1}^{R y_{0}}$ e $v=w_{2}^{R y_{0}}$ em (2.76) e (2.77), respectivamente, implica que

$$
\begin{aligned}
& \quad\left|\int_{\mathbb{R}^{N}}\left(t f\left(w_{1}^{R y_{0}}, w_{2}^{R y_{0}}\right)-f\left(t w_{1}^{R y_{0}}, t w_{2}^{R y_{0}}\right)\right) w_{1}^{R y} \mathrm{~d} x\right| \\
& +\left|\int_{\mathbb{R}^{N}}\left(t g\left(w_{1}^{R y_{0}}, w_{2}^{R y_{0}}\right)-g\left(t w_{1}^{R y_{0}}, t w_{2}^{R y_{0}}\right)\right) w_{2}^{R y} \mathrm{~d} x\right| \\
& =\left|\int_{\mathbb{R}^{N}} h_{1}(t) w_{1}^{R y} \mathrm{~d} x\right|+\left|\int_{\mathbb{R}^{N}} h_{2}(t) w_{2}^{R y} \mathrm{~d} x\right| \\
& \leq|t-1| \int_{\mathbb{R}^{N}}\left\{\left|f\left(w_{1}^{R y_{0}}, w_{2}^{R y_{0}}\right)\right|+C\left(\left(\left(w_{1}^{R y_{0}}\right)^{2}+\left(w_{2}^{R y_{0}}\right)^{2}\right) w_{1}^{R y_{0}}+\left(\left(w_{1}^{R y_{0}}\right)^{2}+\left(w_{2}^{R y_{0}}\right)^{2}\right)^{2} w_{1}^{R y_{0}}\right)\right\} w_{1}^{R y} \mathrm{~d} x \\
& +|t-1| \int_{\mathbb{R}^{N}}\left\{\left|g\left(w_{1}^{R y_{0}}, w_{2}^{R y_{0}}\right)\right|+C\left(\left(\left(w_{1}^{R y_{0}}\right)^{2}+\left(w_{2}^{R y_{0}}\right)^{2}\right) w_{1}^{R y_{0}}+\left(\left(w_{1}^{R y_{0}}\right)^{2}+\left(w_{2}^{R y_{0}}\right)^{2}\right)^{2} w_{1}^{R y_{0}}\right)\right\} w_{2}^{R y} \mathrm{~d} x \\
& =|t-1| \int_{\mathbb{R}^{N}}\left\{f\left(w_{1}^{R y_{0}}, w_{2}^{R y_{0}}\right) w_{1}^{R y}+g\left(w_{1}^{R y_{0}}, w_{2}^{R y_{0}}\right) w_{2}^{R y}\right\} \mathrm{d} x \\
& +|t-1| \int_{\mathbb{R}^{N}}\left\{C\left(\left(\left(w_{1}^{R y_{0}}\right)^{2}+\left(w_{2}^{R y_{0}}\right)^{2}\right) w_{1}^{R y_{0}}+\left(\left(w_{1}^{R y_{0}}\right)^{2}+\left(w_{2}^{R y_{0}}\right)^{2}\right)^{2} w_{1}^{R y_{0}}\right)\right\} w_{1}^{R y} \mathrm{~d} x \\
& +|t-1| \int_{\mathbb{R}^{N}}\left\{C\left(\left(\left(w_{1}^{R y_{0}}\right)^{2}+\left(w_{2}^{R y_{0}}\right)^{2}\right) w_{1}^{R y_{0}}+\left(\left(w_{1}^{R y_{0}}\right)^{2}+\left(w_{2}^{R y_{0}}\right)^{2}\right)^{2} w_{1}^{R y_{0}}\right)\right\} w_{2}^{R y} \mathrm{~d} x .
\end{aligned}
$$

Vamos estimar as integrais em (2.78). Como $\nabla F(u, v):=(f(u, v), g(u, v))$ tem-se

$$
\int_{\mathbb{R}^{N}}\left\{f\left(w_{1}^{R y_{0}}, w_{1}^{R y_{0}}\right) w_{1}^{R y}+g\left(w_{1}^{R y_{0}}, w_{2}^{R y_{0}}\right) w_{2}^{R y}\right\} \mathrm{d} x=\int_{\mathbb{R}^{N}} \nabla F\left(w_{1}^{R y_{0}}, w_{2}^{R y_{0}}\right)\left(w_{1}^{R y}, w_{2}^{R y}\right) \mathrm{d} x=\varepsilon_{R} .
$$


Usando os Lemas 2.12 e 2.14 obtemos

$$
\int_{\mathbb{R}^{N}}\left(\left(w_{1}^{R y_{0}}\right)^{2}+\left(w_{2}^{R y_{0}}\right)^{2}\right) w_{1}^{R y_{0}} w_{1}^{R y} \mathrm{~d} x=\int_{\mathbb{R}^{N}}\left(w_{1}^{R y_{0}}\right)^{3} w_{1}^{R y} \mathrm{~d} x+\int_{\mathbb{R}^{N}}\left(w_{2}^{R y_{0}}\right)^{2} w_{1}^{R y_{0}} w_{1}^{R y} \mathrm{~d} x=O\left(\varepsilon_{R}\right) .
$$

Similarmente, as demais integrais em (2.78) têm a mesma estimativa, isto é,

$$
\begin{aligned}
& \int_{\mathbb{R}^{N}}\left(\left(w_{1}^{R y_{0}}\right)^{2}+\left(w_{2}^{R y_{0}}\right)^{2}\right)^{2} w_{1}^{R y_{0}} w_{1}^{R y} \mathrm{~d} x=O\left(\varepsilon_{R}\right) \\
& \int_{\mathbb{R}^{N}}\left(\left(w_{1}^{R y_{0}}\right)^{2}+\left(w_{2}^{R y_{0}}\right)^{2}\right) w_{2}^{R y_{0}} w_{2}^{R y} \mathrm{~d} x=O\left(\varepsilon_{R}\right) \\
& \int_{\mathbb{R}^{N}}\left(\left(w_{1}^{R y_{0}}\right)^{2}+\left(w_{2}^{R y_{0}}\right)^{2}\right)^{2} w_{2}^{R y_{0}} w_{2}^{R y} \mathrm{~d} x=O\left(\varepsilon_{R}\right) .
\end{aligned}
$$

Reunindo as estimativas em (2.78), (2.79), (2.80) e (2.81), temos a conclusão do corolário.

Lema 2.18. Considere $\beta=\frac{1}{2}$. Se $R \rightarrow+\infty$, então tem-se que

$$
T_{\frac{1}{2}, y}^{R} \rightarrow 2
$$

uniformemente em $y \in \partial B_{2}\left(y_{0}\right)$ onde $y_{0} \in \mathbb{R}^{N}$ é fixado com $\left\|y_{0}\right\|=1$.

Demonstração: Como $\left(w_{1}, w_{2}\right)$ é solução do problema $\left(\mathbb{S}_{\infty}\right)$, então $I_{\infty}^{\prime}\left(w_{1}, w_{2}\right)\left(w_{1}, w_{2}\right)=0$ implicando em

$$
\left\|\left(w_{1}^{R y_{0}}, w_{2}^{R y_{0}}\right)\right\|^{2}-2 \lambda \int_{\mathbb{R}^{N}} w_{2}^{R y_{0}} w_{1}^{R y_{0}} \mathrm{~d} x=\int_{\mathbb{R}^{N}} \nabla F\left(w_{1}^{R y_{0}}, w_{2}^{R y_{0}}\right)\left(w_{1}^{R y_{0}}, w_{2}^{R y_{0}}\right) \mathrm{d} x .
$$

Procedendo de forma análoga obtemos

$$
\left\|\left(w_{1}^{R y}, w_{2}^{R y}\right)\right\|^{2}-2 \lambda \int_{\mathbb{R}^{N}} w_{2}^{R y} w_{1}^{R y} \mathrm{~d} x=\int_{\mathbb{R}^{N}} \nabla F\left(w_{1}^{R y}, w_{2}^{R y}\right)\left(w_{1}^{R y}, w_{2}^{R y}\right) \mathrm{d} x .
$$

Consideramos a combinação linear

$$
Z_{\beta, y}^{R}=\left(\beta w_{1}^{R y_{0}}+(1-\beta) w_{1}^{R y}, \beta w_{2}^{R y_{0}}+(1-\beta) w_{2}^{R y}\right)
$$

e tomando $\beta=\frac{1}{2} \operatorname{temos} Z_{\frac{1}{2}, y}^{R}=\left(\frac{1}{2} w_{1}^{R y_{0}}+\frac{1}{2} w_{1}^{R y}, \frac{1}{2} w_{2}^{R y_{0}}+\frac{1}{2} w_{2}^{R y}\right)$.

Calculando $J$ em $2 Z_{\frac{1}{2}, y}^{R}$ e utilizando (2.82) e (2.83) obtemos 


$$
\begin{aligned}
& J\left(2 Z_{\frac{1}{2}, y}^{R}\right)=J\left(w_{1}^{R y_{0}}+w_{1}^{R y}, w_{2}^{R y_{0}}+w_{2}^{R y}\right) \\
& =\left\|\left(w_{1}^{R y_{0}}+w_{1}^{R y}, w_{2}^{R y_{0}}+w_{2}^{R y}\right)\right\|^{2}-2 \lambda \int_{\mathbb{R}^{N}}\left(w_{1}^{R y_{0}}+w_{1}^{R y}\right)\left(w_{2}^{R y_{0}}+w_{2}^{R y}\right) \mathrm{d} x \\
& -\int_{\mathbb{R}^{N}}(1+a(x)) \nabla F\left(w_{1}^{R y_{0}}+w_{1}^{R y}, w_{2}^{R y_{0}}+w_{2}^{R y}\right)\left(w_{1}^{R y_{0}}+w_{1}^{R y}, w_{2}^{R y_{0}}+w_{2}^{R y}\right) \mathrm{d} x . \\
& =\left\|w_{1}^{R y_{0}}\right\|^{2}+\left\|w_{1}^{R y_{0}}\right\|^{2}+\left\|w_{2}^{R y_{0}}\right\|^{2}+\left\|w_{2}^{R y}\right\|^{2}+2\left\langle w_{1}^{R y_{0}}, w_{1}^{R y}\right\rangle+2\left\langle w_{2}^{R y_{0}}, w_{2}^{R y}\right\rangle \\
& -2 \lambda \int_{\mathbb{R}^{N}}\left(w_{1}^{R y_{0}} w_{2}^{R y_{0}}+w_{1}^{R y_{0}} w_{2}^{R y_{0}}+w_{1}^{R y} w_{2}^{R y_{0}}+w_{1}^{R y} w_{2}^{R y}\right) \mathrm{d} x \\
& -\int_{\mathbb{R}^{N}}(1+a(x)) \nabla F\left(w_{1}^{R y_{0}}+w_{1}^{R y}, w_{2}^{R y_{0}}+w_{2}^{R y}\right)\left(w_{1}^{R y_{0}}+w_{1}^{R y}, w_{2}^{R y_{0}}+w_{2}^{R y}\right) \mathrm{d} x \\
& =\int_{\mathbb{R}^{N}} \nabla F\left(w_{1}^{R y_{0}}, w_{2}^{R y_{0}}\right)\left(w_{1}^{R y_{0}}, w_{2}^{R y_{0}}\right) \mathrm{d} x+\int_{\mathbb{R}^{N}} \nabla F\left(w_{1}^{R y}, w_{2}^{R y}\right)\left(w_{1}^{R y}, w_{2}^{R y}\right) \mathrm{d} x \\
& +2\left\langle w_{1}^{R y_{0}}, w_{1}^{R y}\right\rangle+2\left\langle w_{2}^{R y_{0}}, w_{2}^{R y}\right\rangle-2 \lambda \int_{\mathbb{R}^{N}}\left(w_{1}^{R y_{0}} w_{2}^{R y}+w_{1}^{R y} w_{2}^{R y_{0}}\right) \mathrm{d} x \\
& -\int_{\mathbb{R}^{N}}(1+a(x)) \nabla F\left(w_{1}^{R y_{0}}+w_{1}^{R y}, w_{2}^{R y_{0}}+w_{2}^{R y}\right)\left(w_{1}^{R y_{0}}+w_{1}^{R y}, w_{2}^{R y_{0}}+w_{2}^{R y}\right) \mathrm{d} x \\
& \leq \int_{\mathbb{R}^{N}} \nabla F\left(w_{1}^{R y_{0}}, w_{2}^{R y_{0}}\right)\left(w_{1}^{R y_{0}}, w_{2}^{R y_{0}}\right) \mathrm{d} x+\int_{\mathbb{R}^{N}} \nabla F\left(w_{1}^{R y}, w_{2}^{R y}\right)\left(w_{1}^{R y}, w_{2}^{R y}\right) \mathrm{d} x \\
& +2\left\langle w_{1}^{R y_{0}}, w_{1}^{R y}\right\rangle+2\left\langle w_{2}^{R y_{0}}, w_{2}^{R y}\right\rangle \\
& -\int_{\mathbb{R}^{N}}(1+a(x)) \nabla F\left(w_{1}^{R y_{0}}+w_{1}^{R y}, w_{2}^{R y_{0}}+w_{2}^{R y}\right)\left(w_{1}^{R y_{0}}+w_{1}^{R y}, w_{2}^{R y_{0}}+w_{2}^{R y}\right) \mathrm{d} x \\
& =\int_{\mathbb{R}^{N}} \nabla F\left(w_{1}^{R y_{0}}, w_{2}^{R y_{0}}\right)\left(w_{1}^{R y_{0}}, w_{2}^{R y_{0}}\right) \mathrm{d} x+\int_{\mathbb{R}^{N}} \nabla F\left(w_{1}^{R y}, w_{2}^{R y}\right)\left(w_{1}^{R y}, w_{2}^{R y}\right) \mathrm{d} x \\
& +2\left\langle w_{1}^{R y_{0}}, w_{1}^{R y}\right\rangle+2\left\langle w_{2}^{R y_{0}}, w_{2}^{R y}\right\rangle \\
& -\int_{\mathbb{R}^{N}} \nabla F\left(w_{1}^{R y_{0}}+w_{1}^{R y}, w_{2}^{R y_{0}}+w_{2}^{R y}\right)\left(w_{1}^{R y_{0}}+w_{1}^{R y}, w_{2}^{R y_{0}}+w_{2}^{R y}\right) \mathrm{d} x \\
& -\int_{\mathbb{R}^{N}} a(x) \nabla F\left(w_{1}^{R y_{0}}+w_{1}^{R y}, w_{2}^{R y_{0}}+w_{2}^{R y}\right)\left(w_{1}^{R y_{0}}+w_{1}^{R y}, w_{2}^{R y_{0}}+w_{2}^{R y}\right) \mathrm{d} x \\
& =\int_{\mathbb{R}^{N}} \nabla F\left(w_{1}^{R y_{0}}, w_{2}^{R y_{0}}\right)\left(w_{1}^{R y_{0}}, w_{2}^{R y_{0}}\right) \mathrm{d} x+\int_{\mathbb{R}^{N}} \nabla F\left(w_{1}^{R y}, w_{2}^{R y}\right)\left(w_{1}^{R y}, w_{2}^{R y}\right) \mathrm{d} x \\
& +2\left\langle w_{1}^{R y_{0}}, w_{1}^{R y}\right\rangle+2\left\langle w_{2}^{R y_{0}}, w_{2}^{R y}\right\rangle \\
& -\int_{\mathbb{R}^{N}} \nabla F\left(w_{1}^{R y_{0}}+w_{1}^{R y}, w_{2}^{R y_{0}}+w_{2}^{R y}\right)\left(\left(w_{1}^{R y_{0}}, w_{2}^{R y_{0}}\right)+\left(w_{1}^{R y}+w_{2}^{R y}\right)\right) \mathrm{d} x \\
& -\int_{\mathbb{R}^{N}} a(x) \nabla F\left(w_{1}^{R y_{0}}+w_{1}^{R y}, w_{2}^{R y_{0}}+w_{2}^{R y}\right)\left(w_{1}^{R y_{0}}+w_{1}^{R y}, w_{2}^{R y_{0}}+w_{2}^{R y}\right) \mathrm{d} x \\
& =\int_{\mathbb{R}^{N}} \nabla F\left(w_{1}^{R y_{0}}, w_{2}^{R y_{0}}\right)\left(w_{1}^{R y_{0}}, w_{2}^{R y_{0}}\right) \mathrm{d} x+\int_{\mathbb{R}^{N}} \nabla F\left(w_{1}^{R y}, w_{2}^{R y}\right)\left(w_{1}^{R y}, w_{2}^{R y}\right) \mathrm{d} x \\
& +2\left\langle w_{1}^{R y_{0}}, w_{1}^{R y}\right\rangle_{H^{1}}+2\left\langle w_{2}^{R y_{0}}, w_{2}^{R y}\right\rangle \\
& -\int_{\mathbb{R}^{N}} \nabla F\left(w_{1}^{R y_{0}}+w_{1}^{R y}, w_{2}^{R y_{0}}+w_{2}^{R y}\right)\left(w_{1}^{R y_{0}}, w_{2}^{R y_{0}}\right) \mathrm{d} x \\
& -\int_{\mathbb{R}^{N}} \nabla F\left(w_{1}^{R y_{0}}+w_{1}^{R y}, w_{2}^{R y_{0}}+w_{2}^{R y}\right)\left(w_{1}^{R y}, w_{2}^{R y}\right) \mathrm{d} x \\
& -\int_{\mathbb{R}^{N}} a(x) \nabla F\left(w_{1}^{R y_{0}}+w_{1}^{R y}, w_{2}^{R y_{0}}+w_{2}^{R y}\right)\left(w_{1}^{R y_{0}}+w_{1}^{R y}, w_{2}^{R y_{0}}+w_{2}^{R y}\right) \mathrm{d} x .
\end{aligned}
$$


Ademais,

$$
\nabla F\left(w_{1}^{R y_{0}}+w_{1}^{R y}, w_{2}^{R y_{0}}+w_{2}^{R y}\right)\left(w_{1}^{R y_{0}}, w_{2}^{R y_{0}}\right) \geq \nabla F\left(w_{1}^{R y_{0}}, w_{2}^{R y_{0}}\right)\left(w_{1}^{R y_{0}}, w_{2}^{R y_{0}}\right)
$$

pois como a função $h(x)=\frac{x}{1+s x}$ é crescente para $x>0$ e visto que $w_{i}>0$ para $i=1,2$, tem-se

$$
\begin{aligned}
& \nabla F\left(w_{1}^{R y_{0}}+w_{1}^{R y}, w_{2}^{R y_{0}}+w_{2}^{R y}\right)\left(w_{1}^{R y_{0}}, w_{2}^{R y_{0}}\right) \\
= & f\left(w_{1}^{R y_{0}}+w_{1}^{R y}, w_{2}^{R y_{0}}+w_{2}^{R y}\right) w_{1}^{R y_{0}}+g\left(w_{1}^{R y_{0}}+w_{1}^{R y}, w_{2}^{R y_{0}}+w_{2}^{R y}\right) w_{2}^{R y_{0}} \\
= & \frac{\left(w_{1}^{R y_{0}}+w_{1}^{R y}\right)^{2}+\left(w_{2}^{R y_{0}}+w_{2}^{R y}\right)^{2}}{1+s\left(\left(w_{1}^{R y_{0}}+w_{1}^{R y}\right)^{2}+\left(w_{2}^{R y_{0}}+w_{2}^{R y}\right)^{2}\right)}\left\{\left(w_{1}^{R y_{0}}+w_{1}^{R y}\right) w_{1}^{R y_{0}}+\left(w_{2}^{R y_{0}}+w_{2}^{R y}\right) w_{2}^{R y_{0}}\right\} \\
\geq & \frac{\left(w_{1}^{R y_{0}}\right)^{2}+\left(w_{2}^{R y_{0}}\right)^{2}}{1+s\left(\left(w_{1}^{R y_{0}}\right)^{2}+\left(w_{2}^{R y_{0}}\right)^{2}\right)}\left\{\left(w_{1}^{R y_{0}}\right)^{2}+\left(w_{2}^{R y_{0}}\right)^{2}\right\} \\
= & \nabla F\left(w_{1}^{R y_{0}}, w_{2}^{R y_{0}}\right)\left(w_{1}^{R y_{0}}, w_{2}^{R y_{0}}\right) .
\end{aligned}
$$

Decorre de (2.84) e (2.85) que

$$
\begin{aligned}
J\left(2 Z_{\frac{1}{2}, y}^{R}\right) \leq & 2\left\langle w_{1}^{R y_{0}}, w_{1}^{R y}\right\rangle+2\left\langle w_{2}^{R y_{0}}, w_{2}^{R y}\right\rangle \\
& -\int_{\mathbb{R}^{N}} a(x) \nabla F\left(w_{1}^{R y_{0}}+w_{1}^{R y}, w_{2}^{R y_{0}}+w_{2}^{R y}\right)\left(w_{1}^{R y_{0}}+w_{1}^{R y}, w_{2}^{R y_{0}}+w_{2}^{R y}\right) \mathrm{d} x .
\end{aligned}
$$

Resta estimar o termo integral em (2.86), visto que já sabemos que

$$
\left\langle w_{1}^{R y_{0}}, w_{1}^{R y}\right\rangle=o_{R}(1)=\left\langle w_{2}^{R y_{0}}, w_{2}^{R y}\right\rangle
$$

Utilizando $\left(a_{2}\right)$ e o Lema 2.12 ,

$$
\begin{aligned}
& \int_{\mathbb{R}^{N}}|a(x)| \nabla F\left(w_{1}^{R y_{0}}+w_{1}^{R y}, w_{2}^{R y_{0}}+w_{2}^{R y}\right)\left(w_{1}^{R y_{0}}+w_{1}^{R y}, w_{2}^{R y_{0}}+w_{2}^{R y}\right) \mathrm{d} x \\
& =\int_{\mathbb{R}^{N}}|a(x)| \frac{\left(\left(w_{1}^{R y_{0}}+w_{1}^{R y}\right)^{2}+\left(w_{2}^{R y_{0}}+w_{2}^{R y}\right)^{2}\right)^{2}}{1+s\left(\left(w_{1}^{R y_{0}}+w_{1}^{R y}\right)^{2}+\left(w_{2}^{R y_{0}}+w_{2}^{R y}\right)^{2}\right)} \mathrm{d} x \\
& \leq C \int_{\mathbb{R}^{N}}|a(x)|\left\{\left(w_{1}^{R y_{0}}+w_{1}^{R y}\right)^{4}+\left(w_{2}^{R y_{0}}+w_{2}^{R y}\right)^{4}\right\} \mathrm{d} x=o\left(\varepsilon_{R}\right) .
\end{aligned}
$$

Logo, segue de (2.86), (2.87) e (2.88) que $J\left(2 Z_{\frac{1}{2}, y}^{R}\right) \rightarrow 0$, se $R \rightarrow+\infty$, uniformemente em $y \in \partial B_{2}\left(y_{0}\right)$.

Resta mostrar que

$$
T_{\frac{1}{2}, y}^{R} \rightarrow 2, \text { quando } R \rightarrow \infty
$$

De fato, argumentando por contradição, suponha que existem $\delta>0$ e subsequências $R_{n} \rightarrow \infty$ e $y_{n} \in$ $\partial B_{2}\left(y_{0}\right)$ tais que a sequência

$$
T_{n}:=T_{\frac{1}{2}, y_{n}}^{R_{n}} \quad \text { satisfaz }\left|T_{n}-2\right| \geq \delta .
$$


Como $\left\{T_{n}\right\} \subset \mathbb{R}$ é limitada pelo Lema 2.13, existe uma constante $T$ tal que

$$
T_{n} \rightarrow T \text { quando } n \rightarrow \infty \text {. }
$$

Por $(2.4)$, se $(t u, t v) \in \mathcal{N}, t \in \mathbb{R}^{+}$, então

$$
\|(u, v)\|^{2}-2 \lambda \int_{\mathbb{R}^{N}} u v d x=\int_{\mathbb{R}^{N}}(1+a(x)) \frac{\nabla F(t u, t v)(u, v)}{t} \mathrm{~d} x .
$$

Visto que $T_{\frac{1}{2}, y_{n}}^{R_{n}} Z_{\frac{1}{2}, y_{n}}^{R_{n}} \in \mathcal{N}$, segue de (2.89) que

$$
\begin{aligned}
& \left\|\left(\frac{1}{2} w_{1}^{R_{n} y_{0}}+\frac{1}{2} w_{1}^{R_{n} y_{n}}, \frac{1}{2} w_{2}^{R_{n} y_{0}}+\frac{1}{2} w_{2}^{R_{n} y_{n}}\right)\right\|^{2}-2 \lambda \int_{\mathbb{R}^{N}}\left(\frac{1}{2} w_{1}^{R_{n} y_{0}}+\frac{1}{2} w_{1}^{R_{n} y_{n}}\right)\left(\frac{1}{2} w_{2}^{R_{n} y_{0}}+\frac{1}{2} w_{2}^{R_{n} y_{n}}\right) \mathrm{d} x \\
= & \frac{1}{4} \int_{\mathbb{R}^{N}}(1+a(x)) \frac{\nabla F\left(T_{n}\left(\frac{1}{2} w_{1}^{R_{n} y_{0}}+\frac{1}{2} w_{1}^{R_{n} y_{n}}\right), T_{n}\left(\frac{1}{2} w_{2}^{R_{n} y_{0}}+\frac{1}{2} w_{2}^{R_{n} y_{n}}\right)\right)\left(w_{1}^{R_{n} y_{0}}+w_{1}^{R_{n} y_{n}}, w_{2}^{R_{n} y_{0}}+w_{2}^{R_{n} y_{n}}\right)}{\frac{T_{n}}{2}} .
\end{aligned}
$$

Por um lado, como $\left(w_{1}, w_{2}\right)$ é solução de $\left(\mathbb{S}_{\infty}\right)$ e pela invariância por translação tem-se

$$
\begin{aligned}
\| & \left(\frac{1}{2} w_{1}^{R_{n} y_{0}}+\frac{1}{2} w_{1}^{R_{n} y_{n}}, \frac{1}{2} w_{2}^{R_{n} y_{0}}+\frac{1}{2} w_{2}^{R_{n} y_{n}}\right) \|^{2}-2 \lambda \int_{\mathbb{R}^{N}}\left(\frac{1}{2} w_{1}^{R_{n} y_{0}}+\frac{1}{2} w_{1}^{R_{n} y_{n}}\right)\left(\frac{1}{2} w_{2}^{R_{n} y_{0}}+\frac{1}{2} w_{2}^{R_{n} y_{n}}\right) \mathrm{d} x \\
= & \frac{1}{4}\left\|w_{1}^{R_{n} y_{0}}\right\|^{2}+\frac{1}{4}\left\|w_{1}^{R_{n} y_{n}}\right\|^{2}+\frac{1}{2}\left\langle w_{1}^{R_{n} y_{0}}, w_{1}^{R_{n} y_{n}}\right\rangle+\frac{1}{4}\left\|w_{2}^{R_{n} y_{0}}\right\|^{2}+\frac{1}{4}\left\|w_{2}^{R_{n} y_{n}}\right\|^{2}+\frac{1}{2}\left\langle w_{2}^{R_{n} y_{0}}, w_{2}^{R_{n} y_{n}}\right\rangle \\
& -2 \lambda \int_{\mathbb{R}^{N}}\left(\frac{1}{2} w_{1}^{R_{n} y_{0}}+\frac{1}{2} w_{1}^{R_{n} y_{n}}\right)\left(\frac{1}{2} w_{2}^{R_{n} y_{0}}+\frac{1}{2} w_{2}^{R_{n} y_{n}}\right) \mathrm{d} x \\
= & \frac{1}{4}\left\|w_{1}\right\|^{2}+\frac{1}{4}\left\|w_{1}\right\|^{2}+o_{R_{n}}(1)+\frac{1}{4}\left\|w_{2}\right\|^{2}+\frac{1}{4}\left\|w_{2}\right\|^{2}+o_{R_{n}}(1) \\
& -2 \lambda \int_{\mathbb{R}^{N}}\left(\frac{1}{4} w_{1}^{R_{n} y_{0}} w_{2}^{R_{n} y_{0}}+\frac{1}{4} w_{1}^{R_{n} y_{0}} w_{2}^{R_{n} y_{n}}+\frac{1}{4} w_{1}^{R_{n} y_{n}} w_{2}^{R_{n} y_{0}}+\frac{1}{4} w_{1}^{R_{n} y_{n}} w_{2}^{R_{n} y_{n}}\right) \mathrm{d} x \\
\stackrel{n \rightarrow}{\longrightarrow} \frac{1}{2}\left\|w_{1}\right\|^{2}+\frac{1}{2}\left\|w_{2}\right\|^{2}-2 \lambda \int_{\mathbb{R}^{N}} \frac{2}{4} w_{1} w_{2} \mathrm{~d} x & \\
= & \frac{1}{2}\left\|\left(w_{1}, w_{2}\right)\right\|^{2}-2 \lambda \int_{\mathbb{R}^{N}} \frac{2}{4} w_{1} w_{2} d x=\frac{1}{2} \int_{\mathbb{R}^{N}} \nabla F\left(w_{1}, w_{2}\right)\left(w_{1}, w_{2}\right) \mathrm{d} x .
\end{aligned}
$$

Por outro lado, por $\left(a_{1}\right),\left(F_{1}\right), \quad \nabla F\left(w_{1}, w_{2}\right)\left(w_{1}, w_{2}\right) \leq \frac{w_{1}^{2}(0)+w_{2}^{2}(0)}{s}$ e pelo Teorema da Convergência Dominada de Lebesgue obtemos

$$
\begin{aligned}
& \frac{1}{4} \int_{\mathbb{R}^{N}}(1+a(x)) \frac{\nabla F\left(T_{n}\left(\frac{1}{2} w_{1}^{R_{n} y_{0}}+\frac{1}{2} w_{1}^{R_{n} y_{n}}\right), T_{n}\left(\frac{1}{2} w_{2}^{R_{n} y_{0}}+\frac{1}{2} w_{2}^{R_{n} y_{n}}\right)\right)\left(w_{1}^{R_{n} y_{0}}+w_{1}^{R_{n} y_{n}}, w_{2}^{R_{n} y_{0}}+w_{2}^{R_{n} y_{n}}\right)}{\frac{T_{n}}{2}} \\
& =\frac{1}{4} \int_{\mathbb{R}^{N}} \frac{\nabla F\left(T_{n}\left(\frac{1}{2} w_{1}^{R_{n} y_{0}}+\frac{1}{2} w_{1}^{R_{n} y_{n}}\right), T_{n}\left(\frac{1}{2} w_{2}^{R_{n} y_{0}}+\frac{1}{2} w_{2}^{R_{n} y_{n}}\right)\right)\left[\left(w_{1}^{R_{n} y_{0}}, w_{2}^{R_{n} y_{0}}\right)+\left(w_{1}^{R_{n} y_{n}}, w_{2}^{R_{n} y_{n}}\right)\right]}{\frac{T_{n}}{2}} \\
& +\frac{1}{4} \int_{\mathbb{R}^{N}} a(x) \frac{\nabla F\left(T_{n}\left(\frac{1}{2} w_{1}^{R_{n} y_{0}}+\frac{1}{2} w_{1}^{R_{n} y_{n}}\right), T_{n}\left(\frac{1}{2} w_{2}^{R_{n} y_{0}}+\frac{1}{2} w_{2}^{R_{n} y_{n}}\right)\right)\left[\left(w_{1}^{R_{n} y_{0}}, w_{2}^{R_{n} y_{0}}\right)+\left(w_{1}^{R_{n} y_{n}}, w_{2}^{R_{n} y_{n}}\right)\right]}{\frac{T_{n}}{2}} \\
& \stackrel{n \rightarrow \infty}{\longrightarrow} \frac{2}{4} \int_{\mathbb{R}^{N}} \frac{\nabla F\left(\frac{T}{2}\left(w_{1}, w_{2}\right)\left(w_{1}, w_{2}\right)\right)}{\frac{T}{2}} \mathrm{~d} x .
\end{aligned}
$$


De (2.90), (2.91) e (2.92) temos

$$
\frac{1}{2} \int_{\mathbb{R}^{N}} \nabla F\left(w_{1}, w_{2}\right)\left(w_{1}, w_{2}\right) \mathrm{d} x-\frac{2}{4} \int_{\mathbb{R}^{N}} \frac{\nabla F\left(\frac{T}{2}\left(w_{1}, w_{2}\right)\left(w_{1}, w_{2}\right)\right)}{\frac{T}{2}} \mathrm{~d} x=0 .
$$

Por $\left(F_{1}\right)$ segue que $T=2$. Contradição. Logo,

$$
T_{\frac{1}{2}, y}^{R} \rightarrow 2 \text {, quando } R \rightarrow \infty
$$

e finalizamos a prova do lema.

No próximo resultado demonstraremos uma versão para sistemas como já vimos no Lema 1.14 para o caso escalar.

Lema 2.19. Considere $a, b, c, d$ números reais positivos. Então existe uma constante $C>0$ satisfazendo

$$
\begin{aligned}
& F(a+b, c+d)-F(a, c)-F(b, d)-\nabla F(a, c)(b, d)-\nabla F(b, d)(a, c) \\
\geq & -C\left\{(c d)^{2}+(a b)^{2}+(a d)^{2}+(b c)^{2}\right\} .
\end{aligned}
$$

Demonstração: Seja $t \geq 0$ e a função real $j(t):=F(a+t b, c+t d)$. Observe que $j$ é crescente. Defina $G(t)=j^{\prime}(t)=\nabla F(a+t b, c+t d)(b, d)$. Note que

$$
\begin{aligned}
G(t) & =\frac{(a+t b)^{2}+(c+t d)^{2}}{\left[1+s\left((a+t b)^{2}+(c+t d)^{2}\right)\right]}(a+t b) b+(c+t d) d \\
& :=\frac{Z^{2}(t)}{1+s Z^{2}(t)}[(a+t b) b+(c+t d) d] .
\end{aligned}
$$

Derivando $G(t)$ temos para todo $t \geq 0$

$$
\begin{aligned}
G^{\prime}(t)= & \frac{2 Z Z^{\prime}\left(1+s Z^{2}\right)-Z^{2} \cdot 2 s Z Z^{\prime}}{\left(1+s Z^{2}\right)^{2}}[(a+t b) b+(c+t d) d] \\
& +\frac{Z^{2}(t)}{1+s Z^{2}(t)}\left(b^{2}+d^{2}\right) \\
= & \frac{2 Z Z^{\prime}}{\left(1+s Z^{2}\right)^{2}}[(a+t b) b+(c+t d) d] \\
& +\frac{Z^{2}(t)}{1+s Z^{2}(t)}\left(b^{2}+d^{2}\right)>0
\end{aligned}
$$

logo $j^{\prime}$ é crescente. Pelo Teorema Fundamental do Cálculo e o crescimento de $j^{\prime}$ já verificado tem-se

$$
\begin{aligned}
& F(a+b, c+d)-F(a, c)-\nabla F(a, c)(b, d) \\
= & j(1)-j(0)-j^{\prime}(0) \\
= & \int_{0}^{1} j^{\prime}(t) \mathrm{d} t-j^{\prime}(0) \\
\geq & \int_{0}^{1} j^{\prime}(0) \mathrm{d} t-j^{\prime}(0)=0 .
\end{aligned}
$$


Agora vamos estimar $-F(b, d)-\nabla F(b, d)(a, c)$. Defina $\psi(t)=F(t b, t d)$. Então $\psi^{\prime}(t)=\nabla F(t b, t d)(b, d)$, $\psi(0)=F(0,0)=0$ e $\psi(1)=F(b, d)$. Utilizando o Teorema Fundamental do Cálculo segue que

$$
\begin{aligned}
& -F(b, d)-\nabla F(b, d)(a, c) \\
= & -\int_{0}^{1} \psi^{\prime}(t) \mathrm{d} t-\nabla F(b, d)(a, c) \\
\geq & -\int_{0}^{1} \frac{(t b)^{2}+(t d)^{2}}{1+s\left((t b)^{2}+(t d)^{2}\right)}\left(t b^{2}+t d^{2}\right) \mathrm{d} t-\frac{b^{2}+d^{2}}{1+s\left(b^{2}+d^{2}\right)}(b a+d c) \\
\geq & -\int_{0}^{1} t^{3}\left(b^{2}+d^{2}\right)^{2} \mathrm{~d} t-\left(b^{2}+d^{2}\right)(b a+d c) \\
= & -\frac{\left(b^{2}+d^{2}\right)^{2}}{4}-\left(b^{2}+d^{2}\right)(b a+d c) \\
= & -\frac{1}{4}\left\{b^{4}+d^{4}+2 b^{2} d^{2}+4 a b^{3}+4 b^{2} c d+4 a b d^{2}+4 c d^{3}\right\} .
\end{aligned}
$$

Considere $0<b \leq a$ e $0<d \leq c$. Note que

i) $b^{4}+4 a b^{3}=(a b)^{2}\left\{\frac{b^{2}}{a^{2}}+\frac{4 b}{a}\right\} \leq(a b)^{2}\left\{\frac{b^{2}}{a^{2}}+\frac{4 a}{a}\right\} \leq 5(a b)^{2}$;

ii) $d^{4}+4 c d^{3} \leq 5(c d)^{2}$, analogamente;

iii) $5(a b)^{2}+4 a b d^{2}=(a d)^{2}\left\{\frac{5 b^{2}}{d^{2}}+\frac{4 b}{a}\right\} \leq(a d)^{2}\left\{\frac{5 b^{2}}{d^{2}}+4\right\} \leq 5(a b)^{2}+4(a d)^{2}$;

iv) $2(b d)^{2}+4 b^{2} c d=2(b c)^{2}\left\{\frac{d^{2}}{c^{2}}+2 \frac{d}{c}\right\} \leq 2(b c)^{2}\{1+2\} \leq 6(b c)^{2}$.

De $(i)-(i v)$ segue que $(2.94)$ resulta em

$$
-F(b, d)-\nabla F(b, d)(a, c) \geq-\frac{1}{4}\left\{5(c d)^{2}+5(a b)^{2}+4(a d)^{2}+6(b c)^{2}\right\} .
$$

O caso em que $0<a \leq b$ e $0<c \leq d$ é análogo (simétrico). Portanto, segue de (2.93) e (2.95) o resultado.

Lema 2.20. Existem números $R_{3}>0, \eta_{1}>0$ tais que

$$
I\left(T_{\beta, y}^{R} Z_{\beta, y}^{R}\right) \leq 2 m_{\infty}-\eta_{1}
$$

para cada $R>R_{3}$ e para todo $y \in \partial B_{2}\left(y_{0}\right)$ e $\beta \in[0,1]$.

Demonstração: Inicialmente vamos escrever a combinação linear na forma simplificada $T_{\beta, y}^{R} Z_{\beta, y}^{R}=\left(\beta T_{\beta, y}^{R} w_{1}^{R y_{0}}+(1-\beta) T_{\beta, y}^{R} w_{1}^{R y}, \beta T_{\beta, y}^{R} w_{2}^{R y_{0}}+(1-\beta) T_{\beta, y}^{R} w_{2}^{R y}\right)=\left(t_{1} w_{1}^{R y_{0}}+t_{2} w_{1}^{R y}, t_{1} w_{2}^{R y_{0}}+t_{2} w_{2}^{R y}\right)$. 
Além disso, tomando $u=t_{1} w_{1}^{R y_{0}}+t_{2} w_{1}^{R y}$ e $v=t_{1} w_{2}^{R y_{0}}+t_{2} w_{2}^{R y}$ em (2.1) segue que

$$
\begin{aligned}
& I\left(t_{1} w_{1}^{R y_{0}}+t_{2} w_{1}^{R y}, t_{1} w_{2}^{R y_{0}}+t_{2} w_{2}^{R y}\right) \\
& =\frac{1}{2}\left\|\left(t_{1} w_{1}^{R y_{0}}+t_{2} w_{1}^{R y}, t_{1} w_{2}^{R y_{0}}+t_{2} w_{2}^{R y}\right)\right\|^{2}-\lambda \int_{\mathbb{R}^{N}}\left(t_{1} w_{1}^{R y_{0}}+t_{2} w_{1}^{R y}\right)\left(t_{1} w_{2}^{R y_{0}}+t_{2} w_{2}^{R y}\right) \mathrm{d} x \\
& -\int_{\mathbb{R}^{N}}(1+a(x)) F\left(t_{1} w_{1}^{R y_{0}}+t_{2} w_{1}^{R y}, t_{1} w_{2}^{R y_{0}}+t_{2} w_{2}^{R y}\right) \mathrm{d} x \\
& =\frac{1}{2} \int_{\mathbb{R}^{N}}\left[t_{1}^{2}\left|\nabla w_{1}^{R y_{0}}\right|^{2}+t_{2}^{2}\left|\nabla w_{1}^{R y}\right|^{2}+2 t_{1} t_{2} \nabla w_{1}^{R y_{0}} \nabla w_{1}^{R y}+\left(t_{1} w_{1}^{R y_{0}}+t_{2} w_{1}^{R y}\right)^{2}\right] \mathrm{d} x \\
& +\frac{1}{2} \int_{\mathbb{R}^{N}}\left[t_{1}^{2}\left|\nabla w_{2}^{R y_{0}}\right|^{2}+t_{2}^{2}\left|\nabla w_{2}^{R y}\right|^{2}+2 t_{1} t_{2} \nabla w_{2}^{R y_{0}} \nabla w_{2}^{R y}+\left(t_{1} w_{2}^{R y_{0}}+t_{2} w_{2}^{R y}\right)^{2}\right] \mathrm{d} x \\
& -\lambda \int_{\mathbb{R}^{N}}\left[t_{1}^{2} w_{1}^{R y_{0}} w_{2}^{R y}+t_{1} t_{2} w_{1}^{R y_{0}} w_{2}^{R y}+t_{1} t_{2} w_{1}^{R y} w_{2}^{R y_{0}}+t_{2}^{2} w_{1}^{R y} w_{2}^{R y}\right] \mathrm{d} x \\
& -\int_{\mathbb{R}^{N}}(1+a(x)) F\left(t_{1} w_{1}^{R y_{0}}+t_{2} w_{1}^{R y}, t_{1} w_{2}^{R y_{0}}+t_{2} w_{2}^{R y}\right) \mathrm{d} x \\
& (A)=\frac{t_{1}^{2}}{2} \int_{\mathbb{R}^{N}}\left[\left|\nabla w_{1}^{R y_{0}}\right|^{2}+\left(w_{1}^{R y_{0}}\right)^{2}+\left|\nabla w_{2}^{R y_{0}}\right|^{2}+\left(w_{2}^{R y_{0}}\right)^{2}\right]-\lambda t_{1}^{2} \int_{\mathbb{R}^{N}} w_{1}^{R y_{0}} w_{2}^{R y_{0}} \mathrm{~d} x \\
& \text { (B) } \quad-\int_{\mathbb{R}^{N}} F\left(t_{1} w_{1}^{R y_{0}}, t_{1} w_{2}^{R y_{0}}\right) \mathrm{d} x \\
& \text { (C) } \quad+\frac{t_{2}^{2}}{2} \int_{\mathbb{R}^{N}}\left[\left|\nabla w_{1}^{R y}\right|^{2}+\left(w_{1}^{R y}\right)^{2}+\left|\nabla w_{2}^{R y}\right|^{2}+\left(w_{2}^{R y}\right)^{2}\right]-\lambda t_{2}^{2} \int_{\mathbb{R}^{N}} w_{1}^{R y} w_{2}^{R y} \mathrm{~d} x \\
& \text { (D) } \quad-\int_{\mathbb{R}^{N}} F\left(t_{2} w_{1}^{R y}, t_{2} w_{2}^{R y}\right) \mathrm{d} x \\
& \text { (E) } \quad+t_{1} t_{2} \int_{\mathbb{R}^{N}}\left[\nabla w_{1}^{R y_{0}} \nabla w_{1}^{R y}+w_{1}^{R y_{0}} w_{1}^{R y}+\nabla w_{2}^{R y_{0}} \nabla w_{2}^{R y}+w_{2}^{R y_{0}} w_{2}^{R y}\right] \mathrm{d} x \\
& \text { (F) } \quad-\lambda t_{1} t_{2} \int_{\mathbb{R}^{N}}\left(w_{1}^{R y_{0}} w_{2}^{R y}+w_{2}^{R y_{0}} w_{1}^{R y}\right) \mathrm{d} x \\
& \text { (G) } \quad-\int_{\mathbb{R}^{N}}\left[\nabla F\left(t_{1} w_{1}^{R y_{0}}, t_{1} w_{2}^{R y_{0}}\right)\left(t_{2} w_{1}^{R y}, t_{2} w_{2}^{R y}\right)+\nabla F\left(t_{2} w_{1}^{R y}, t_{2} w_{2}^{R y}\right)\left(t_{1} w_{1}^{R y_{0}}, t_{1} w_{2}^{R y_{0}}\right)\right] \mathrm{d} x \\
& (H) \quad+\int_{\mathbb{R}^{N}}\left[\nabla F\left(t_{1} w_{1}^{R y_{0}}, t_{1} w_{2}^{R y_{0}}\right)\left(t_{2} w_{1}^{R y}, t_{2} w_{2}^{R y}\right)+\nabla F\left(t_{2} w_{1}^{R y}, t_{2} w_{2}^{R y}\right)\left(t_{1} w_{1}^{R y_{0}}, t_{1} w_{2}^{R y_{0}}\right)\right] \mathrm{d} x \\
& \text { (I) } \quad-\int_{\mathbb{R}^{N}}\left[F\left(t_{1} w_{1}^{R y_{0}}+t_{2} w_{1}^{R y}, t_{1} w_{2}^{R y_{0}}+t_{2} w_{2}^{R y}\right)-F\left(t_{1} w_{1}^{R y_{0}}, t_{1} w_{2}^{R y_{0}}\right)-F\left(t_{1} w_{1}^{R y}, t_{1} w_{2}^{R y}\right)\right] \mathrm{d} x \\
& (J) \quad-\int_{\mathbb{R}^{N}} a(x) F\left(t_{1} w_{1}^{R y_{0}}+t_{2} w_{1}^{R y}, t_{1} w_{2}^{R y_{0}}+t_{2} w_{2}^{R y}\right) \mathrm{d} x .
\end{aligned}
$$

Vamos estimar cada uma das linha de $(A)$ a $(J)$. Para as linhas $(A)$ e $(B)$, desde que $\left(w_{1}, w_{2}\right)$ são soluções de energia mínima e pela invariância por translação nas integrais do funcional $I_{\infty}$ temos que

$$
(A)+(B)=I_{\infty}\left(t_{1} w_{1}^{R y_{0}}, t_{1} w_{2}^{R y_{0}}\right) \leq m_{\infty} .
$$

Analogamente para as linhas $(C)$ e $(D)$ temos

$$
(C)+(D)=I_{\infty}\left(t_{2} w_{1}^{R y_{0}}, t_{2} w_{2}^{R y_{0}}\right) \leq m_{\infty} .
$$


A linha $(I)$ pode ser estimada pelo Lema 2.19

$$
\begin{aligned}
& F\left(t_{1} w_{1}^{R y_{0}}+t_{2} w_{1}^{R y}, t_{1} w_{2}^{R y_{0}}+t_{2} w_{2}^{R y}\right)-F\left(t_{1} w_{1}^{R y_{0}}, t_{1} w_{2}^{R y_{0}}\right)-F\left(t_{2} w_{1}^{R y}, t_{2} w_{2}^{R y}\right) \\
- & \nabla F\left(t_{1} w_{1}^{R y_{0}}, t_{1} w_{2}^{R y_{0}}\right)\left(t_{2} w_{1}^{R y}, t_{2} w_{2}^{R y}\right)-\nabla F\left(t_{2} w_{1}^{R y}, t_{2} w_{2}^{R y}\right)\left(t_{1} w_{1}^{R y_{0}}, t_{1} w_{2}^{R y_{0}}\right) \\
\geq & -C\left\{\left(t_{1} w_{2}^{R y_{0}} t_{2} w_{2}^{R y}\right)^{2}+\left(t_{1} w_{1}^{R y_{0}} t_{2} w_{1}^{R y}\right)^{2}+\left(t_{1} w_{1}^{R y_{0}} t_{2} w_{2}^{R y}\right)^{2}+\left(t_{2} w_{1}^{R y} t_{1} w_{2}^{R y_{0}}\right)^{2}\right\} .
\end{aligned}
$$

Integrando e invertendo os sinais temos

$$
\begin{aligned}
& -\int_{\mathbb{R}^{N}}\left[F\left(t_{1} w_{1}^{R y_{0}}+t_{2} w_{1}^{R y}, t_{1} w_{2}^{R y_{0}}+t_{2} w_{2}^{R y}\right)-F\left(t_{1} w_{1}^{R y_{0}}, t_{1} w_{2}^{R y_{0}}\right)-F\left(t_{2} w_{1}^{R y}, t_{2} w_{2}^{R y}\right)\right] \mathrm{d} x \\
& \leq- \\
& \quad-\int_{\mathbb{R}^{N}}\left[\nabla F\left(t_{1} w_{1}^{R y_{0}}, t_{1} w_{2}^{R y_{0}}\right)\left(t_{2} w_{1}^{R y}, t_{2} w_{2}^{R y}\right)+\nabla F\left(t_{2} w_{1}^{R y}, t_{2} w_{2}^{R y}\right)\left(t_{1} w_{1}^{R y_{0}}, t_{1} w_{2}^{R y_{0}}\right)\right] \mathrm{d} x \\
& \quad+C \int_{\mathbb{R}^{N}}\left\{\left(t_{1} w_{2}^{R y_{0}} t_{2} w_{2}^{R y}\right)^{2}+\left(t_{1} w_{1}^{R y_{0}} t_{2} w_{1}^{R y}\right)^{2}+\left(t_{1} w_{1}^{R y_{0}} t_{2} w_{2}^{R y}\right)^{2}+\left(t_{2} w_{1}^{R y} t_{1} w_{2}^{R y_{0}}\right)^{2}\right\} \mathrm{d} x .
\end{aligned}
$$

Utilizando os Lemas 2.12 e 2.13, equação $(2.73),\left(a_{2}\right)$ e tomando $\mu_{0}$ tal que $\sqrt{1-\lambda}<\mu_{0}<2 \sqrt{1-\lambda}$, segue que

$$
\begin{aligned}
\int_{\mathbb{R}^{N}}\left(t_{1} w_{2}^{R y_{0}} t_{2} w_{2}^{R y}\right)^{2} d x & \leq C\left(t_{1} t_{2}\right)^{2} \int_{\mathbb{R}^{N}} e^{-2 \sqrt{1-\lambda}\left|x-R y_{0}\right|} e^{-2 \sqrt{1-\lambda}|x-R y|} \mathrm{d} x \\
& \leq C\left(t_{1} t_{2}\right)^{2} \int_{\mathbb{R}^{N}} e^{-\mu_{0}\left|x-R y_{0}\right|} e^{-2 \sqrt{1-\lambda}|x-R y|} \mathrm{d} x \\
& \leq C\left(t_{1} t_{2}\right)^{2} e^{-\mu_{0}\left|R y_{0}-R y\right|} \leq C\left(t_{1} t_{2}\right)^{2} e^{-2 \mu_{0} R}=o\left(\varepsilon_{R}\right)
\end{aligned}
$$

e analogamente

$$
C \int_{\mathbb{R}^{N}}\left\{\left(t_{1} w_{1}^{R y_{0}} t_{2} w_{1}^{R y}\right)^{2}+\left(t_{1} w_{1}^{R y_{0}} t_{2} w_{2}^{R y}\right)^{2}+\left(t_{2} w_{1}^{R y} t_{1} w_{2}^{R y_{0}}\right)^{2}\right\} \mathrm{d} x=o\left(\varepsilon_{R}\right) .
$$

Logo, de (2.99), (2.100) e (2.101) segue que

$$
(H)+(I) \leq o\left(\varepsilon_{R}\right)
$$

Vamos estimar a linha $(J)$. Utilizando os Lemas 2.12 e 2.13 , equação $(2.73),\left(a_{2}\right)$ e para $i, j=1,2$

$$
\int_{\mathbb{R}^{N}}|a(x)|\left(t_{i} w_{i}^{R y_{0}}\right)^{4} d x \leq o\left(\varepsilon_{R}\right)
$$

e

$$
\int_{\mathbb{R}^{N}}|a(x)|\left(t_{j} w_{i}^{R y}\right)^{4} \mathrm{~d} x \leq o\left(\varepsilon_{R}\right)
$$


Observando que $a(x)$ pode mudar de sinal, segue de $(2.103),(2.104)$ e $\left(F_{2}\right)$ que

$$
\begin{aligned}
(J) & \leq \int_{\mathbb{R}^{N}}|a(x)| F\left(t_{1} w_{1}^{R y_{0}}+t_{2} w_{1}^{R y}, t_{1} w_{2}^{R y_{0}}+t_{2} w_{2}^{R y}\right) \mathrm{d} x \\
& \leq \frac{1}{2} \int_{\mathbb{R}^{N}}|a(x)| \nabla F\left(t_{1} w_{1}^{R y_{0}}+t_{2} w_{1}^{R y}, t_{1} w_{2}^{R y_{0}}+t_{2} w_{2}^{R y}\right)\left(t_{1} w_{1}^{R y_{0}}+t_{2} w_{1}^{R y}, t_{1} w_{2}^{R y_{0}}+t_{2} w_{2}^{R y}\right) \mathrm{d} x \\
& =\frac{1}{2} \int_{\mathbb{R}^{N}}|a(x)| \frac{\left(\left(t_{1} w_{1}^{R y_{0}}+t_{2} w_{1}^{R y}\right)^{2}+\left(t_{1} w_{2}^{R y_{0}}+t_{2} w_{2}^{R y}\right)^{2}\right)^{2}}{1+s\left(\left(t_{1} w_{1}^{R y_{0}}+t_{2} w_{1}^{R y}\right)^{2}+\left(t_{1} w_{2}^{R y_{0}}+t_{2} w_{2}^{R y}\right)^{2}\right)} \mathrm{d} x \\
& \leq \frac{1}{2} \int_{\mathbb{R}^{N}}|a(x)|\left(\left(t_{1} w_{1}^{R y_{0}}+t_{2} w_{1}^{R y}\right)^{2}+\left(t_{1} w_{2}^{R y_{0}}+t_{2} w_{2}^{R y}\right)^{2}\right)^{2} \mathrm{~d} x \\
& \leq C \int_{\mathbb{R}^{N}}|a(x)|\left(\left(t_{1} w_{1}^{R y_{0}}+t_{2} w_{1}^{R y}\right)^{4}+\left(t_{1} w_{2}^{R y_{0}}+t_{2} w_{2}^{R y}\right)^{4}\right)^{\mathrm{d} x} \\
& \leq C \int_{\mathbb{R}^{N}}|a(x)|\left(\left(t_{1} w_{1}^{R y_{0}}\right)^{4}+\left(t_{2} w_{1}^{R y}\right)^{4}+\left(t_{1} w_{2}^{R y_{0}}\right)^{4}+\left(t_{2} w_{2}^{R y}\right)^{4}\right) \mathrm{d} x \leq o\left(\varepsilon_{R}\right) .
\end{aligned}
$$

Resta estimar $(E),(F),(G)$. Temos dois casos a analisar: $\beta$ em um pequeno intervalo contendo $1 / 2 \mathrm{e}$ $\beta$ nos intervalos complementares em $[0,1]$. Quando $\beta=1 / 2$, utilizando que $\left(w_{1}, w_{2}\right)$ é solução de $\left(\mathbb{S}_{\infty}\right)$, o Corolário 2.17 e o Lema 2.16 com $t_{1}, t_{2} \geq 1 / 2, \bar{C}_{0}>0$ e $C>0$ temos que

$$
\begin{aligned}
& (E)+(F)+(G) \\
= & t_{1} t_{2} \int_{\mathbb{R}^{N}}\left[\nabla w_{1}^{R y_{0}} \nabla w_{1}^{R y}+w_{1}^{R y_{0}} w_{1}^{R y}+\nabla w_{2}^{R y_{0}} \nabla w_{2}^{R y}+w_{2}^{R y_{0}} w_{2}^{R y}\right] \mathrm{d} x \\
& \lambda t_{1} t_{2} \int_{\mathbb{R}^{N}}\left(w_{1}^{R y_{0}} w_{2}^{R y}+w_{2}^{R y_{0}} w_{1}^{R y}\right) \mathrm{d} x \\
& -\int_{\mathbb{R}^{N}}\left[\nabla F\left(t_{1} w_{1}^{R y_{0}}, t_{1} w_{2}^{R y_{0}}\right)\left(t_{2} w_{1}^{R y}, t_{2} w_{2}^{R y}\right)+\nabla F\left(t_{2} w_{1}^{R y}, t_{2} w_{2}^{R y}\right)\left(t_{1} w_{1}^{R y_{0}}, t_{1} w_{2}^{R y_{0}}\right)\right] \mathrm{d} x \\
= & \frac{t_{1} t_{2}}{2} \int_{\mathbb{R}^{N}}\left[\nabla w_{1}^{R y_{0}} \nabla w_{1}^{R y}+w_{1}^{R y_{0}} w_{1}^{R y}+\nabla w_{2}^{R y_{0}} \nabla w_{2}^{R y}+w_{2}^{R y_{0}} w_{2}^{R y}\right] \mathrm{d} x \\
& +\frac{t_{1} t_{2}}{2} \int_{\mathbb{R}^{N}}\left[\nabla w_{1}^{R y_{0}} \nabla w_{1}^{R y}+w_{1}^{R y_{0}} w_{1}^{R y}+\nabla w_{2}^{R y_{0}} \nabla w_{2}^{R y}+w_{2}^{R y_{0}} w_{2}^{R y}\right] \mathrm{d} x \\
& -\frac{\lambda}{2} t_{1} t_{2} \int_{\mathbb{R}^{N}}\left(w_{1}^{R y_{0}} w_{2}^{R y}+w_{2}^{R y_{0}} w_{1}^{R y}\right) \mathrm{d} x \\
& -\frac{\lambda}{2} t_{1} t_{2} \int_{\mathbb{R}^{N}}\left(w_{1}^{R y_{0}} w_{2}^{R y}+w_{2}^{R y_{0}} w_{1}^{R y}\right) \mathrm{d} x \\
& -\int_{\mathbb{R}^{N}}\left[\nabla F\left(t_{1} w_{1}^{R y_{0}}, t_{1} w_{2}^{R y_{0}}\right)\left(t_{2} w_{1}^{R y}, t_{2} w_{2}^{R y}\right)+\nabla F\left(t_{2} w_{1}^{R y}, t_{2} w_{2}^{R y}\right)\left(t_{1} w_{1}^{R y_{0}}, t_{1} w_{2}^{R y_{0}}\right)\right] \mathrm{d} x \\
= & \frac{t_{2}}{2} \int_{\mathbb{R}^{N}} t_{1} \nabla F\left(w_{1}^{R y_{0}}, w_{2}^{R y_{0}}\right)\left(w_{1}^{R y}, w_{2}^{R y}\right) \mathrm{d} x+\frac{t_{1}}{2} \int_{\mathbb{R}^{N}} t_{2} \nabla F\left(w_{1}^{R y}, w_{2}^{R y}\right)\left(w_{1}^{R y_{0}}, w_{2}^{R y_{0}}\right) \mathrm{d} x \\
& -\int_{\mathbb{R}^{N}}\left[\nabla F\left(t_{1} w_{1}^{R y_{0}}, t_{1} w_{2}^{R y_{0}}\right)\left(t_{2} w_{1}^{R y}, t_{2} w_{2}^{R y}\right)+\nabla F\left(t_{2} w_{1}^{R y}, t_{2} w_{2}^{R y}\right)\left(t_{1} w_{1}^{R y_{0}}, t_{1} w_{2}^{R y_{0}}\right)\right] \mathrm{d} x
\end{aligned}
$$




$$
\begin{aligned}
= & \frac{t_{2}}{2} \int_{\mathbb{R}^{N}}\left\{t_{1} \nabla F\left(w_{1}^{R y_{0}}, w_{2}^{R y_{0}}\right)\left(w_{1}^{R y}, w_{2}^{R y}\right)-\nabla F\left(t_{1} w_{1}^{R y_{0}}, t_{1} w_{2}^{R y_{0}}\right)\left(w_{1}^{R y}, w_{2}^{R y}\right)\right\} \mathrm{d} x \\
& +\frac{t_{1}}{2} \int_{\mathbb{R}^{N}}\left\{t_{2} \nabla F\left(w_{1}^{R y}, w_{2}^{R y}\right)\left(w_{1}^{R y_{0}}, w_{2}^{R y_{0}}\right)-\nabla F\left(t_{2} w_{1}^{R y}, t_{2} w_{2}^{R y}\right)\left(w_{1}^{R y_{0}}, w_{2}^{R y_{0}}\right)\right\} \mathrm{d} x \\
& -\frac{1}{2} \int_{\mathbb{R}^{N}}\left[\nabla F\left(t_{1} w_{1}^{R y_{0}}, t_{1} w_{2}^{R y_{0}}\right)\left(t_{2} w_{1}^{R y}, t_{2} w_{2}^{R y}\right)+\nabla F\left(t_{2} w_{1}^{R y}, t_{2} w_{2}^{R y}\right)\left(t_{1} w_{1}^{R y_{0}}, t_{1} w_{2}^{R y_{0}}\right)\right] \mathrm{d} x \\
= & \frac{t_{2}}{2}\left|\int_{\mathbb{R}^{N}}\left(t_{1} f\left(w_{1}^{R y_{0}}, w_{2}^{R y_{0}}\right)-f\left(t_{1} w_{1}^{R y_{0}}, t_{1} w_{2}^{R y_{0}}\right)\right) w_{1}^{R y} \mathrm{~d} x\right| \\
& +\frac{t_{2}}{2}\left|\int_{\mathbb{R}^{N}}\left(t_{1} g\left(w_{1}^{R y_{0}}, w_{2}^{R y_{0}}\right)-g\left(t_{1} w_{1}^{R y_{0}}, t_{1} w_{2}^{R y_{0}}\right)\right) w_{2}^{R y} \mathrm{~d} x\right| \\
& +\frac{t_{1}}{2}\left|\int_{\mathbb{R}^{N}}\left(t_{2} f\left(w_{1}^{R y}, w_{2}^{R y}\right)-f\left(t_{2} w_{1}^{R y}, t_{2} w_{2}^{R y}\right)\right) w_{1}^{R y_{0}} \mathrm{~d} x\right| \\
& +\frac{t_{1}}{2}\left|\int_{\mathbb{R}^{N}}\left(t_{2} g\left(w_{1}^{R y}, w_{2}^{R y}\right)-g\left(t_{2} w_{1}^{R y}, t_{2} w_{2}^{R y}\right)\right) w_{2}^{R y_{0}} \mathrm{~d} x\right| \\
& -\frac{1}{2} \int_{\mathbb{R}^{N}}\left[\nabla F\left(t_{1} w_{1}^{R y_{0}}, t_{1} w_{2}^{R y_{0}}\right)\left(t_{2} w_{1}^{R y}, t_{2} w_{2}^{R y}\right)+\nabla F\left(t_{2} w_{1}^{R y}, t_{2} w_{2}^{R y}\right)\left(t_{1} w_{1}^{R y_{0}}, t_{1} w_{2}^{R y_{0}}\right)\right] \mathrm{d} x \\
\leq & \frac{t_{2}}{2} C\left|t_{1}-1\right| O\left(\varepsilon_{R}\right)+\frac{t_{1}}{2} C\left|t_{2}-1\right| O\left(\varepsilon_{R}\right) \\
& -\frac{1}{2} \int_{\mathbb{R}^{N}}\left[\nabla F\left(t_{1} w_{1}^{R y_{0}}, t_{1} w_{2}^{R y_{0}}\right)\left(t_{2} w_{1}^{R y}, t_{2} w_{2}^{R y}\right)+\nabla F\left(t_{2} w_{1}^{R y}, t_{2} w_{2}^{R y}\right)\left(t_{1} w_{1}^{R y_{0}}, t_{1} w_{2}^{R y_{0}}\right)\right] \mathrm{d} x \\
\leq & \frac{t_{2}}{2} C\left|t_{1}-1\right| O\left(\varepsilon_{R}\right)+\frac{t_{1}}{2} C\left|t_{2}-1\right| O\left(\varepsilon_{R}\right)-\bar{C}_{0} \varepsilon_{R} . \\
&
\end{aligned}
$$

Portanto,

$$
(E)+(F)+(G) \leq \frac{t_{2}}{2} C\left|t_{1}-1\right| O\left(\varepsilon_{R}\right)+\frac{t_{1}}{2} C\left|t_{2}-1\right| O\left(\varepsilon_{R}\right)-\bar{C}_{0} \varepsilon_{R}
$$

Como $0<t_{1}, t_{2} \leq T_{0}$ e $t_{1}(R) \rightarrow 1, t_{2}(R) \rightarrow 1$, se $R \rightarrow \infty$, pelo Lema 2.18, então tomando $R_{0}>0$ suficientemente grande e $\delta \in\left(0, \frac{1}{2}\right)$ suficientemente pequeno, temos que

$$
(E)+(F)+(G) \leq-\frac{\bar{C}_{0}}{2} \varepsilon_{R}
$$

para todo $\beta \in\left[\frac{1}{2}-\delta, \frac{1}{2}+\delta\right], y \in B_{2}\left(y_{0}\right)$ e $R \geq R_{0}$. Assim, por (2.97), (2.98), (2.102), (2.105) e (2.107), para todo $\beta \in\left[\frac{1}{2}-\delta, \frac{1}{2}+\delta\right], y \in B_{2}\left(y_{0}\right)$ e $R \geq R_{0}$, provamos que

$$
I\left(t_{1} w_{1}^{R y_{0}}+t_{2} w_{1}^{R y}, t_{1} w_{2}^{R y_{0}}+t_{2} w_{2}^{R y}\right) \leq 2 m_{\infty}-\frac{\bar{C}_{0}}{2} \varepsilon_{R}+o\left(\varepsilon_{R}\right)
$$

Observe que neste argumento utilizamos a continuidade de $T_{\beta, y}^{R}$ nas variáveis $\beta, y$ e $R$.

Finalmente, estudamos o segundo caso $\beta \in\left[0, \frac{1}{2}-\delta\right) \cup\left(\frac{1}{2}+\delta, 1\right]$. Para isto, sem perda de generalidade, fixemos $\beta$ tal que $0 \leq \beta<\frac{1}{2}-\delta$. Então $1 \geq 1-\beta>\frac{1}{2}+\delta$. Note que, se $T_{\beta, y}^{R} \leq 2$, então $t_{1}=T_{\beta, y}^{R} \beta \in[0,1-2 \delta]$ e $t_{2}=T_{\beta, y}^{R}(1-\beta) \in[1+2 \delta, 2]$, ou seja, temos $t_{1}<1$ e $t_{2}>1$. Por outro lado, se $T_{\beta, y}^{R} \geq 2$, então $t_{1}=T_{\beta, y}^{R} \beta \in[1-2 \delta,+\infty]$ e $t_{2}=T_{\beta, y}^{R}(1-\beta) \in[1+2 \delta,+\infty]$. Juntando isto e as estimativas anteriores de (2.97) a (2.105) e o Lema 2.4-(c) mostramos que

$$
I\left(t_{1} w_{1}^{R y_{0}}+t_{2} w_{1}^{R y}, t_{1} w_{2}^{R y_{0}}+t_{2} w_{2}^{R y}\right) \leq m_{\infty}-\gamma+m_{\infty}-\gamma+O\left(\varepsilon_{R}\right) .
$$


$\forall \beta \in\left[0, \frac{1}{2}-\delta\right] \cup\left[\frac{1}{2}+\delta, 1\right], y \in B_{2}\left(y_{0}\right)$ e $R \geq R_{1}$.

Portanto, concluímos o lema juntando os resultados em (2.108) e (2.109) para todo $\beta \in[0,1], y \in \partial B_{2}\left(y_{0}\right), R>\max \left\{R_{0}, R_{1}\right\}$.

Lema 2.21. Dado número real $\eta_{2}>0$, existe um número real $R_{4}>0$ tal que

$$
I\left(T_{0, y}^{R} Z_{0, y}^{R}\right)<m_{\infty}+\eta_{2}
$$

para todo $y \in \partial B_{2}\left(y_{0}\right)$ e $R>R_{4}$. Em particular, $m \leq m_{\infty}$

Demonstração: Começamos observando que, pelo Lema 2.12 e a equação (2.73), para $i=1,2$ já vimos em (2.105) que

$$
\int_{\mathbb{R}^{N}}|a(x)| F\left(T_{0, y}^{R} w_{1}^{R y}, T_{0, y}^{R} w_{2}^{R y}\right) \mathrm{d} x \leq o\left(\varepsilon_{R}\right) .
$$

Escolhendo $(u, v)=\left(T_{0, y}^{R} w_{1}^{R y}, T_{0, y}^{R} w_{2}^{R y}\right)=Z_{0, y}^{R}$ em (2.6) e utilizando o Lema 2.4 e a desigualdade (2.110) temos

$$
\begin{aligned}
I\left(T_{0, y}^{R} w_{1}^{R y}, T_{0, y}^{R} w_{2}^{R y}\right) & =I_{\infty}\left(T_{0, y}^{R} w_{1}^{R y}, T_{0, y}^{R} w_{2}^{R y}\right)-\int_{\mathbb{R}^{N}} a(x) F\left(T_{0, y}^{R} w_{1}^{R y}, T_{0, y}^{R} w_{2}^{R y}\right) \mathrm{d} x \\
& \leq \max _{t>0} I_{\infty}\left(t w_{1}^{R y}, t w_{2}^{R y}\right)+\int_{\mathbb{R}^{N}}|a(x)| F\left(T_{0, y}^{R} w_{1}^{R y}, T_{0, y}^{R} w_{2}^{R y}\right) \mathrm{d} x \\
& =I_{\infty}\left(w_{1}^{R y}, w_{2}^{R y}\right)+\int_{\mathbb{R}^{N}}|a(x)| F\left(T_{0, y}^{R} w_{1}^{R y}, T_{0, y}^{R} w_{2}^{R y}\right) \mathrm{d} x \\
& =m_{\infty}+\int_{\mathbb{R}^{N}}|a(x)| F\left(T_{0, y}^{R} w_{1}^{R y}, T_{0, y}^{R} w_{2}^{R y}\right) \mathrm{d} x \leq m_{\infty}+o\left(\varepsilon_{R}\right)
\end{aligned}
$$

Assim, o resultado segue e o lema está demonstrado.

\subsection{Demonstração do resultado principal do sistema}

Para a demonstração do Teorema 0.2 serão utilizadas ferramentas do tipo min-max. Precisamos introduzir a função baricentro em $H^{1}\left(\mathbb{R}^{N}\right)$ dada em [16]. Sejam $u \in H^{1}\left(\mathbb{R}^{N}\right) \backslash\{0\}$,

$$
\mu(u)(x):=\frac{1}{\mu\left(B_{1}(0)\right)} \int_{B_{1}(x)}|u(y)| \mathrm{d} y, \quad \mu(u) \in L^{\infty} \text { e contínua }
$$

e

$$
\bar{u}(x):=\left(\mu(u)(x)-\frac{1}{2} \max _{x \in \mathbb{R}^{N}} \mu(u)(x)\right)^{+}, \bar{u} \in C_{0}\left(\mathbb{R}^{N}\right)
$$

onde $\mu\left(B_{1}(0)\right)$ é a medida de Lebesgue da bola unitária. A função baricentro de $u$ é a aplicação definida por

$$
\overline{\mathcal{B}}(u):=\frac{1}{|\bar{u}|_{1}} \int_{\mathbb{R}^{N}} x \bar{u}(x) \mathrm{d} x .
$$

A função baricentro possui as seguintes propriedades: 
i) $\overline{\mathcal{B}}$ é contínua em $H^{1}\left(\mathbb{R}^{N}\right) \backslash\{0\}$;

ii) Se $u \in H^{1}\left(\mathbb{R}^{N}\right) \backslash\{0\}$ é radialmente simétrica, então $\overline{\mathcal{B}}(u)=0$;

iii) Para todo $t \in \mathbb{R} \backslash\{0\}$ e todo $u \in H^{1}\left(\mathbb{R}^{N}\right) \backslash\{0\}$ tem-se $\overline{\mathcal{B}}(u)=\overline{\mathcal{B}}(t u)$;

iv) Dado $z \in \mathbb{R}^{N}$ tem-se $\overline{\mathcal{B}}(u(x-z))=\overline{\mathcal{B}}(u)+z$.

Agora consideramos a definição da função baricentro do par $(u, v) \in H \backslash\{(0,0)\}$ dada em [5]:

$$
\mathcal{B}(u, v):=\frac{1}{|\bar{u}|_{1}+|\bar{v}|_{1}} \int_{\mathbb{R}^{N}} x(\bar{u}(x)+\bar{v}(x)) \mathrm{d} x \in \mathbb{R}^{N} .
$$

Convém salientar que, se $u, v \neq 0$, então

$$
\mathcal{B}(u, v):=\frac{|\bar{u}|_{1}}{|\bar{u}|_{1}+|\bar{v}|_{1}} \overline{\mathcal{B}}(u)+\frac{|\bar{v}|_{1}}{|\bar{u}|_{1}+|\bar{v}|_{1}} \overline{\mathcal{B}}(v) .
$$

Assim, $\mathcal{B}$ está bem definida e verifica propriedades análogas as (i)-(iv).

Definida a função baricentro, podemos demonstrar o seguinte resultado:

Lema 2.22. Suponha que $m$ não seja atingido. Então $m=m_{\infty}$ e existe um $\eta>0$ tal que

$$
\mathcal{B}(u, v) \neq 0, \text { para toda }(u, v) \in \mathcal{N} \cap I^{m_{\infty}+\eta}
$$

onde denotamos

$$
I_{\infty}^{m_{\infty}+\eta}:=\left\{(u, v) \in H^{1}\left(\mathbb{R}^{N}\right) \times H^{1}\left(\mathbb{R}^{N}\right) ; \quad I(u, v) \leq m_{\infty}+\eta\right\}
$$

Demonstração: Suponha que $m$ não é atingido. Pelo Lema 2.11, $m \geq m_{\infty}$ e do Lema 2.21, a desigualdade invertida. Logo temos a igualdade $m=m_{\infty}$.

Argumentando por contradição, suponha que para cada $n \in \mathbb{N}$ exista $\left\{\left(\varphi_{n}, \psi_{n}\right)\right\} \subset \mathcal{N}$ satisfazendo $I\left(\varphi_{n}, \psi_{n}\right)<m+o_{n}(1)$ e, ainda, $\mathcal{B}\left(\varphi_{n}, \psi_{n}\right)=0$. Segue disto que $\left\{\left(\varphi_{n}, \psi_{n}\right)\right\} \subset \mathcal{N}$ é uma sequência minimizante para $I$. O Princípio Variacional de Ekeland, por instante vide [46], nos fornece uma sequência $\left\{\left(u_{n}, v_{n}\right)\right\}$ para $I$ restrito a $\mathcal{N}$ no nível $m_{\infty}$ onde tal sequência é (PS) e satisfaz

$$
\left\|\left(\varphi_{n}, \psi_{n}\right)-\left(u_{n}, v_{n}\right)\right\| \rightarrow 0, \text { quando } n \rightarrow \infty
$$

Já vimos pelos Lemas 2.5 e 2.7 que toda sequência (PS) em $\mathcal{N}$ é limitada e como $m$ não é atingido, podemos usar o Lema 2.10 para garantir a existência de uma sequência

$$
\left\{z_{n}\right\} \subset \mathbb{R}^{N} ;\left\|z_{n}\right\| \rightarrow \infty \text { e }\left\|\left(u_{n}, v_{n}\right)-\left(w_{1}\left(\cdot-z_{n}\right), w_{2}\left(\cdot-z_{n}\right)\right)\right\| \rightarrow 0, \text { quando } n \rightarrow \infty
$$

onde $\left(w_{1}, w_{2}\right)$ é a solução positiva, radial de energia mínima do problema limite.

Por translação temos

$$
u_{n}\left(x+z_{n}\right)=w_{1}(x)+o_{n}(1) \text { e } v_{n}\left(x+z_{n}\right)=w_{2}(x)+o_{n}(1) .
$$

Usando as propriedades da função baricentro e que $\mathcal{B}\left(\varphi_{n}, \psi_{n}\right)=0$ temos

$$
\mathcal{B}\left(\varphi_{n}\left(x+z_{n}\right), \psi_{n}\left(x+z_{n}\right)\right)=\mathcal{B}\left(\varphi_{n}, \psi_{n}\right)+z_{n}=z_{n}
$$


e ainda pela continuidade da função baricentro $\mathcal{B}$ na norma em $H$

$$
\mathcal{B}\left(\varphi_{n}\left(x+z_{n}\right), \psi_{n}\left(x+z_{n}\right)\right) \rightarrow \mathcal{B}\left(w_{1}(x), w_{2}(x)\right)=0
$$

que configura uma contradição, pois $\left\|z_{n}\right\| \rightarrow \infty$.

Por fim iremos demonstrar o resultado principal onde utilizaremos os resultados que obtivemos anteriormente.

\section{Prova do Teorema 0.2 :}

Inicialmente, suponha que $m$ é atingido para $\operatorname{algum}(u, v)$ em $\mathcal{N}$. Consequentemente, pelos Lemas $2.4(\mathrm{~b})$ e 2.6 temos que $(u, v)$ é uma solução não trivial para o problema $(\mathbb{S})$. Suponha então que $m$ não seja atingido. Segue do Lema 2.22 que $m=m_{\infty}$ e podemos fixar $\eta_{2} \in\left(0, \frac{m_{\infty}}{8}\right)$ tal que $I(u, v) \leq$ $m_{\infty}+\eta_{2}, \quad \forall(u, v) \in \mathcal{N}$ e $\mathcal{B}(u, v) \neq 0$ e ainda, pelo Lema 2.21 , para todo $R>R_{4}$

$$
I\left(T_{0, y}^{R} Z_{0, y}^{R}\right) \leq m_{\infty}+\eta_{2}, \quad \forall y \in \partial B_{2}\left(y_{0}\right)
$$

Segue do Lema 2.20 que podemos escolher $\eta_{1}$ entre $\left(0, \frac{m_{\infty}}{8}\right)$ e para todo $R>R_{3}$

$$
I\left(T_{\beta, y}^{R} Z_{\beta, y}^{R}\right) \leq 2 m_{\infty}-\eta_{1}, \quad \forall y \in \partial B_{2}\left(y_{0}\right), \quad \beta \in[0,1]
$$

Fixe $R>\max \left\{R_{3}, R_{4}\right\}$ e defina a seguinte aplicação

$$
\begin{array}{cl}
\mathcal{H}: B_{2}\left(y_{0}\right) & \longrightarrow \mathcal{N} \cap I^{2 m_{\infty}-\eta_{1}} \\
\beta y_{0}+(1-\beta) y & \longrightarrow T_{\beta, y}^{R} Z_{\beta, y}^{R}, \quad \forall y \in \partial B_{2}\left(y_{0}\right), \quad \beta \in[0,1] .
\end{array}
$$

O objetivo agora é mostrar que o funcional $I$ tem um valor crítico no intervalo $\left(m_{\infty}, 2 m_{\infty}\right)$. Argumentando por contradição, suponha que tal valor crítico não exista. Como $m$ não é atingido, podemos utilizar o Lema 2.11 para garantir que o funcional $I$ restrito a $\mathcal{N}$ satisfaz a condição de Palais- Smale no intervalo $\left(m_{\infty}, 2 m_{\infty}\right)$. Logo, existe um $\varepsilon>0$ tal que

$$
\left\|\left(\left.I\right|_{\mathcal{N}}\right)^{\prime}(u, v)\right\| \geq \varepsilon \text { para todo } \quad(u, v) \in \mathcal{N} \cap I^{-1}\left[m_{\infty}+\eta_{2}, 2 m_{\infty}-\eta_{1}\right]
$$

Isso implica que, pelo Lema 5.15 [46], existe uma aplicação (deformação) contínua

$$
\mathcal{D}: \mathcal{N} \cap I^{2 m_{\infty}-\eta_{1}} \longrightarrow \mathcal{N} \cap I^{m_{\infty}+\eta_{2}}
$$

tal que $\mathcal{D}=i d$ (aplicação identidade) para todo $(u, v) \in \mathcal{N} \cap I^{m_{\infty}+\eta_{2}}$.

Por $(2.111),(2.112),(2.113)$ e (2.114) podemos definir a seguinte aplicação contínua

$$
\begin{aligned}
& \Gamma: B_{2}(0) \quad \longrightarrow \quad \partial B_{2}(0)
\end{aligned}
$$

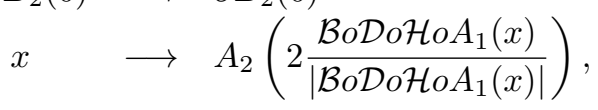


onde $\mathcal{B}$ é a aplicação baricentro e as aplicações contínuas $A_{1}$ e $A_{2}$ são definidas como segue

$$
\begin{array}{ccc}
A_{1}: B_{2}(0) & \longrightarrow & B_{2}\left(y_{0}\right) \\
x & \longrightarrow & x+y_{0}
\end{array}
$$

e

$$
\begin{aligned}
& A_{2}: \partial B_{2}(0) \quad \longrightarrow \quad \partial B_{2}(0) \\
& \frac{2 y}{|y|} \quad \longrightarrow \quad y-y_{0}
\end{aligned}
$$

onde $y \in \partial B_{2}\left(y_{0}\right)$. Além disso, se $\beta=0$ segue que

$$
\mathcal{H}(y)=T_{0, y}^{R} Z_{0, y}^{R}
$$

Por outro lado, usando as propriedades da função baricentro note que

$$
\mathcal{B}\left(T_{0, y}^{R} Z_{0, y}^{R}\right)=\mathcal{B}\left(T_{0, y}^{R}\left(\omega_{1}^{R y}, \omega_{2}^{R y}\right)\right)=\mathcal{B}\left(\omega_{1}^{R y}, \omega_{2}^{R y}\right)=\mathcal{B}\left(\omega_{1}(x), \omega_{2}(x)\right)+R y=R y .
$$

Como $\mathcal{D}=i d$ para todo $(u, v) \in \mathcal{N} \cap I^{m_{\infty}+\eta_{2}}$ tem-se por (2.115), (2.116), (2.117), (2.118) e (2.119) que

$$
\Gamma\left(y-y_{0}\right)=A_{2}\left(2 \frac{\mathcal{B} o \mathcal{D} o \mathcal{H} o A_{1}\left(y-y_{0}\right)}{\left|\mathcal{B} o \mathcal{D} o \mathcal{H} o A_{1}\left(y-y_{0}\right)\right|}\right)=A_{2}\left(2 \frac{\mathcal{B}\left(T_{0, y}^{R} z_{0, y}^{R}\right)}{\left|\mathcal{B}\left(T_{0, y}^{R} z_{0, y}^{R}\right)\right|}\right)=A_{2}\left(2 \frac{R y}{|R y|}\right)=y-y_{0}
$$

Temos uma contradição, pois a aplicação contínua $\Gamma$ não pode existir pelo Teorema do Ponto Fixo de Brouwer, veja Teorema 3.5 em [7]. Portanto, a contradição neste caso veio de supor que $I$ não tem um valor crítico no intervalo $\left(m_{\infty}, 2 m_{\infty}\right)$.

Para discutir a existência de uma solução positiva $u>0, v>0$, consideremos o funcional

$$
I_{+}(u, v)=\frac{1}{2}\|(u, v)\|_{H}^{2}-\lambda \int_{\mathbb{R}^{N}} u v \mathrm{~d} x-\int_{\mathbb{R}^{N}}(1+a(x)) F\left(u^{+}, v^{+}\right) \mathrm{d} x .
$$

Para maiores detalhes sobre este funcional $I_{+}$, veja a Proposição 1.12 em [46].

Aplicando os mesmos argumentos anteriores do funcional $I$, pelo Teorema 0.2 podemos obter uma solução do problema

$$
\left(\mathbb{S}_{+}\right)\left\{\begin{array}{l}
-\Delta u+u=(1+a(x)) \frac{u^{+}\left(\left(u^{+}\right)^{2}+\left(v^{+}\right)^{2}\right)}{1+s\left(\left(u^{+}\right)^{2}+\left(v^{+}\right)^{2}\right)}+\lambda v \quad \text { em } \quad \mathbb{R}^{N}, \\
-\Delta v+v=(1+a(x)) \frac{v^{+}\left(\left(u^{+}\right)^{2}+\left(v^{+}\right)^{2}\right)}{1+s\left(\left(u^{+}\right)^{2}+\left(v^{+}\right)^{2}\right)}+\lambda u \text { em } \quad \mathbb{R}^{N} .
\end{array}\right.
$$

Somando as duas equações do sistema $\left(\mathbb{S}_{+}\right)$temos

$$
-\Delta(u+v)+(1-\lambda)(u+v)=(1+a(x)) \frac{u^{+}\left(\left(u^{+}\right)^{2}+\left(v^{+}\right)^{2}\right)}{1+s\left(\left(u^{+}\right)^{2}+\left(v^{+}\right)^{2}\right)}+(1+a(x)) \frac{v^{+}\left(\left(u^{+}\right)^{2}+\left(v^{+}\right)^{2}\right)}{1+s\left(\left(u^{+}\right)^{2}+\left(v^{+}\right)^{2}\right)} .
$$

Desde que $0<\lambda<1$, por $\left(a_{1}\right)$ e pelo Princípio do Máximo temos que $u+v>0$. 
Adicionando $\lambda u$ a ambos os lados da primeira equação em $\left(\mathbb{S}_{+}\right)$temos

$$
-\Delta u+(1+\lambda) u=(1+a(x)) \frac{u^{+}\left(\left(u^{+}\right)^{2}+\left(v^{+}\right)^{2}\right)}{1+s\left(\left(u^{+}\right)^{2}+\left(v^{+}\right)^{2}\right)}+\lambda(u+v)>0 .
$$

Novamente por $\left(a_{1}\right)$ e pelo Princípio do Máximo temos $u>0$. De maneira análoga, $v>0$. Assim, $(u, v)$ é uma solução positiva de $\left(\mathbb{S}_{+}\right)$. 


\section{Referências Bibliográficas}

[1] Ackermann, N.; Clapp, M. and Pacella, F., Alternating sign multibump solutions of nonlinear elliptic equations in expanding tubular domains, Comm. Partial Differential Equations. 38 (2013), 751-779.

[2] Akhmediev, N.; Królinowski, W. and Snyder, A., Partially coherent solitons of variable shape, Physical Review Letters 81, no. 21,(1998), 4632-4635.

[3] Akhmediev, N. and Ankiewicz, A., Novel soliton states and bifurcation phenomena in nonlinear fiber couplers, Phys. Rev. Lett. 70, 2395-2398.

[4] Ambrosetti, A., Remarks on some systems of nonlinear Schrödinger equations, Journal of Fixed Point Theory and Applications 4 (2008), 35-46.

[5] Ambrosetti , A.; Cerami, G. and Ruiz, D., Solitions of linearly coupled systems of semilinear nonautonomous equations on $\mathbb{R}^{N}$, Journal of Functional Analysis. 254 (2008), 2816-2845.

[6] Ambrosetti , A.; Colorado, E. and Ruiz, D., Multi-bump solitons to linearly coupled systems of nonlinear Schrödinger equations, Calc. Var. Partial Diff. Equations, 2007 , 85-112.

[7] Ambrosetti , A. and Malchiodi, A., Nonlinear analysis and semilinear elliptic problems, Cambridge University Press, 2007.

[8] Bahri, A. and Lions, P.L, On the existence of a positive solution of semilinear elliptic equations in unbound domain, Ann. Inst. Henri Poincaré. 14 (1997), 365-413.

[9] Bahri, A. and Li, Y. Y, On a minimax procedure for the existence of a positive solution for certain scalar field, Revista Mat. Iberoamericana. 6 (1997), 1-2.

[10] Bartolo, P.; Benci, V. and Fortunato, D., Abstract critical point theorems and applications to some nonlinear problems with strong resonance at infinity, Nonlinear Analysis: Theory, Methods Applications. 7 (1983), 981-1012.

[11] Beitia, J. B.; García, V. M. P. and Torres, P. J.; Solitary waves for linearly coupled nonlinear Schrödinger equations with inhomogeneous coefficientes, Nonlinear Science 19 (2009), 437-451.

[12] Berestycki, H.; Gallouet, T. and Kavian, O., Equations de champs scalaires euclidiens non lineaires das le plan, C. R. Acad. Sci. Paris Ser. I Math. 297 (1983), 305-310. 
[13] Berestycki, H. and Lions, P. L., Nonlinear scalar field equations. I. Existence of a ground state, Arch. Rational Mech. Anal. 82 (1983), 313-345.

[14] Busca, J. and Sirakov, B., Symmetry results for semilinear elliptic systems in the whole space, Journal of Diff. Equations. 163 (2000), 41-56.

[15] Cerami, G. and Passaseo, D., Existence and multiplicity results for semilinear elliptic Dirichlet problems in exterior domains, Nonlinear Analysis, Theory, Methods Applications, 24, (1995), 15331547.

[16] Cerami, G. and Passasseo, D., The effect of concentrating potentials in some singularly perturbed problems, Calc. Var. Partial Differential Equations. 3 (2003), 257-281.

[17] Chen, Z. and Zou, W., Standing waves for a coupled system of nonlinear Schrödinger equations , Annali di Matematica 194 (2015) 183-220.

[18] Clapp, M. and Maia, L. A., A positive bound state for an asymptotically linear or superlinear Schrödinger equations, J. Differential Equation. 260 (2016), 3173-3192.

[19] Coddington, E. A. and Levinson, N.;, Theory of ordinary differential equations, New York: McGrawHill. (1955).

[20] Coti-Zelati, V. and Rabinowitz,P.; Homoclinic type solutions for a semilinear elliptic PDE on $\mathbb{R}^{N}$, Comm. Pure Appl. Math, 46 (1992), 1217-1269.

[21] De Figueiredo, D. G. and Yang, J., Decay, symmetry and existence of solutions of semilinear elliptic systems, Nonlinear Anal. 33 (1998), no. 3, 211-234.

[22] De Figueiredo, D. G. and Mitidieri, E., Maximum principles for linear elliptic systems, Rend. Istit. Mat. Univ. Trieste 22 (1990), no. 1-2, 36-66 (1992).

[23] Ding, W. Y. and Ni, W. M., On the existence of positive entire solutions of a semilinear elliptic equation, Arch. Rational Mech. Anal. 91, no. 4,(1986), 283-308.

[24] Évéquoz, G. and Weth, T., Entire solutions to nonlinear scalar field equations with indefinite linear part, Adv. Nonlinear Studies. 12 (2016), 281-314.

[25] Gidas, B., Ni, W. and Niremberg, L., Symmetry of positive solutions of nonlinear elliptic equations, Math. Analysis and Applications, Part A . Advances in Math. Supplementary Studies, vol 7A., 1981.

[26] Kwong, M. K., Uniquess of positive solutions of $\Delta u-u+u^{p}=0$ in $\mathbb{R}^{N}$, Arch. Rational Mech. Anal 105, (1989), 243-266 .

[27] Lions, P. L., The concentration-compactness principle in the calculus of variations. The locally compact case, Ann. I. H. Poincaré AN 1, (1984), 109 - 145 and 223-283 .

[28] Lehrer, R., Existence of solution for asymptotically linear systems in $\mathbb{R}^{N}$, Electronic Journal of Diferential Equations, 236 (2013), 1-20.

[29] Lehrer, R. and Maia, L. A., Positive solutions of asymptotically linear equations via Pohozaev manifold, Journal Functional Analysis . 266 (2014), 213-246. 
[30] Litchinister, N. M.; Królikowski, W.; Akhmediev, N. N. and Agrawal, G. P., Asymmetric partially coherent solitions in saturable nonlinear media, Phys. Rev. E 60 (1999), 2377-2380.

[31] Maia, L. A. and Pellacci, B. , Positive solutions for asymptotically linear problems in exterior domains, Annali di Matematica Pura ed Applicata . (2016), 1-32.

[32] Maia, L. A., Montefusco, E. and Pellacci, B., Weakly coupled nonlinear Schrödinger systems:the saturation effect, Calc. Var. (2013), 25-351.

[33] Maia, L. A. and Ruviaro, R., Positive and nodal solutions of nonlinear Schrondinger equations in a saturable medium, Adv. Nonlinear Stud. . 15 (2015), 191-219.

[34] Matthews, M.R.; Anderson, B.P.; Haljan, P.C.; Hall, D.S.; Wieman, C.E. and Cornell, E.A., Vortices in a Bose-Einstein condensate, Phys. Rev. Lett. 83, 2498-2501 (1999a).

[35] Matthews, M.R.; Anderson, B.P. Haljan, P.C.; Hall, D.S.; Holland, M.J.; Williams, J.E.; Wieman, C.E. and Cornell, E.A., Watching a superfluid untwist itself: Recurrence of Rabi oscillations in a Bose-Einstein condensate, Phys. Rev. Lett. 83, 3358 (1999b).

[36] McLeod, K., Uniqueness of positive radial solutions of $\Delta u+f(u)=0$ in $\mathbb{R}^{N}$ II, Trans. Amer. Math. Soc. . 339 (1993), 495-505.

[37] Moroz,V. and Schaftingen, J. V., Nonexistence and optimal decay of supersolutions to Choquard equations in exterior domains, Journal of Differential Equations. 254 (2013), 3089-3145.

[38] Moroz,V. and Schaftingen, J. V., Goundstates of nonlinear Choquard equations : Existence, qualitative properties and decay asymptotics, Journal of Funct. Anal. 265 (2013), 153-184.

[39] Rabinowitz, P. H. On a class of nonlinear Schrödinger equations, Z. Angew. Math. Phys. 43 (1992), no. 2, 270-291.

[40] Serrin, J. and Tang, M. Uniquess of ground states for quasilinear elliptic equations, Indiana Univ. Math. 49 (2000), 897-923.

[41] Steman, G. I. and Segev, M., Optical spatial solutions and their interactions:universality and diversity, Science 286 (1999), 1518-1523.

[42] Strauss, W. A. Existence of solitary waves in higher dimensions, Commun. Math. Phys. . 55 (1977), 149-162.

[43] Stuart, C. A., Bifurcation in $L^{p}\left(\mathbb{R}^{N}\right)$ for a semilinear elliptic equation, London. Math. Soc., 57 (1987), 511-541.

[44] Stuart, C. A. Guidance properties of nonlinear planar waveguides, Arch. Rational Mech. Anal. 125 (1993), no. 2, 145-200.

[45] Weilnau, C.; Ahles, M.; Petter, J.; Träger, D.; Schröder, J. and Denz, C., Spatial optical (2+1)dimensional scalar-and vector-solitons in saturable nonlinear media, Ann. Phys. (Leipzig) 11 (2002), 573-629.

[46] Willem, M., Minimax Theorems, Volume 24, Birkhauser, Boston, 1996. 
[47] Zafrany, A.; Malomed, B.A. and Merhasin, I.M., Solitons in a linearly coupled system with separated dispersion and nonlinearity, Chaos 15, 037108 (2005).

[48] Zhang, H.; Xu, J. and Zhang, F., Existence of positive ground states for some nonlinear Schrödinger systems, Boundary Value Problems. 13 (2013). 